\title{
A multi-dimensional spectral description of ocean variability with applications
}

by

\author{
Cimarron James Lemuel Wortham, IV \\ B.A., Reed College (2004)
}

Submitted in partial fulfillment of the requirements for the degree of Doctor of Philosophy

at the

MASSACHUSETTS INSTITUTE OF TECHNOLOGY

and the

\section{WOODS HOLE OCEANOGRAPHIC INSTITUTION}

February 2013

(c)Cimarron James Lemuel Wortham, MMXIII. All rights reserved.

The author hereby grants to MIT and to WHOI permission to reproduce and distribute publicly paper and electronic copies of this thesis document in whole or in part.

Author

Joint Program in Physical Oceanography Massachusetts Institute of Technology and Woods Hole Oceanographic Institution September 14, 2012

Certified by ....

Carl I. Wunsch

Cecil and Ida Green Professor of Physical Oceanography Thesis Supervisor Accepted by

Glenn R. Flierl Chair, Joint Committee for Physical Oceanography 


\title{
A multi-dimensional spectral description of ocean variability with applications
}

by

\author{
Cimarron James Lemuel Wortham, IV
}

Submitted to the Joint Program in Physical Oceanography

Massachusetts Institute of Technology and Woods Hole Oceanographic Institution on September 14, 2012, in partial fulfillment of the

requirements for the degree of

Doctor of Philosophy

\begin{abstract}
Efforts to monitor the ocean for signs of climate change are hampered by ever-present noise, in the form of stochastic ocean variability, and detailed knowledge of the character of this noise is necessary for estimating the significance of apparent trends. Typically, uncertainty estimates are made by a variety of ad hoc methods, often based on numerical model results or the variability of the data set being analyzed. We provide a systematic approach based on the four-dimensional frequency-wavenumber spectrum of low-frequency ocean variability. This thesis presents an empirical model of the spectrum of ocean variability for periods between about 20 days and 15 years and wavelengths of about 200-10,000 km, and describes applications to ocean circulation trend detection, observing system design, and satellite data processing.

The horizontal wavenumber-frequency part of the model spectrum is based on satellite altimetry, current meter data, moored temperature records, and shipboard ADCP data. The spectrum is dominated by motions along a "nondispersive line". The observations considered are consistent with a universal $\omega^{-2}$ power law at the high end of the frequency range, but inconsistent with a universal wavenumber power law. The model spectrum is globally varying and accounts for changes in dominant phase speed, period, and wavelength with location. The vertical structure of the model spectrum is based on numerical model results, current meter data, and theoretical considerations. We find that the vertical structure of kinetic energy is surface intensified relative to the simplest theoretical predictions. We present a theory for the interaction of linear Rossby waves with rough topography; rough topography can explain both the observed phase speeds and vertical structure of variability. The improved description of low-frequency ocean variability presented here will serve as a useful tool for future oceanographic studies.
\end{abstract}

Thesis Supervisor: Carl I. Wunsch

Title: Cecil and Ida Green Professor of Physical Oceanography 


\section{Acknowledgments}

First and foremost, I offer my sincere thanks to my advisor, Carl Wunsch. He provided the inspiration for this thesis, constant questioning that pushed me to explore difficult ideas, and allowed me the freedom to study many avenues of interest.

I would also like to thank my thesis committee. Raffaele Ferrari, Glenn Flierl, and Steven Jayne provided useful comments throughout the process. In particular, I appreciate their guidance in focusing the topic of this thesis. Breck Owens kindly agreed to serve as the Chair of my thesis defense.

Many other scientists have provided assistance and inspiration along the way. Tom Farrar was extremely helpful in explaining the details of the satellite altimetry data. Dimitris Menemenlis, Chris Hill, and Hong Zhang advised me in the analysis of the ECCO2 model. Without them I would have been lost. Discussions with Patrick Heimbach inspired some of the applications of the model spectrum.

When I started in the MIT-WHOI Joint Program, I had no idea how important my fellow students would be to my time here. Ryan Abernathey, Brian Rose, Malte Jansen, Matt Mazloff, and Ross Tulloch were always available to discuss science, and have kept me inspired along the way. Martha Buckley and Jareth Holt provided useful comments on early drafts. I would also like to thank my classmates, Holly Dail, Rachel Horwitz, Rebecca Dell, and Pat Engel. Without them, the long first years of classes would have been much less fun.

I am unspeakably grateful to my parents, Robin and Kansas, for all their love and support over the years. I don't know how you turned me into a scientist, but I'm glad you did. Marissa, you have been my source of balance. As a traveling partner, you have reminded me to take time away from school and have fun. The rest of the time, you remind me to stay focused. I can't imagine the last few years without you, and I look forward to many more.

I have made so many friends during my time in Cambridge, and you have all made it memorable. Ryan, Laura, Chiara, Brian: I simply can't imagine my time at MIT without you. I will always remember the varied discussions with the lunchtime crowd 
in the Charney Library. Andrew, Christina, Zach, Joe, Keith, Nick, biking and racing with you has been amazing.

The altimeter products were produced by SSALTO/DUACS and distributed by AVISO, with support from CNES. Moored current meter and temperature sensor data were provided by Robert Scott and Darran Furnival through the Global MultiArchive Current Meter Database. This research was supported by NASA under grants NNG06GC28G and NNX08AR33G. 


\section{Contents}

$\begin{array}{ll}\text { List of Figures } & 9\end{array}$

1 Introduction $\quad 21$

1.1 Theoretical background . . . . . . . . . . . . . . 25

1.2 Previous work . . . . . . . . . . . . . . . . 31

1.3 Outline . . . . . . . . . . . . . . . . . . 33

1.4 Questions raised . . . . . . . . . . . . . . . 35

2 Observed SSH $k, l, \omega$ spectrum $\quad 37$

2.1 Data and methods . . . . . . . . . . . . . . . . . . 39

2.2 Observed spectrum from altimetry . . . . . . . . . . . . . 44

2.3 Global results . . . . . . . . . . . . . . . . . . . 59

2.4 Nonlinearity ....................... 63

2.5 Conclusions ....................... 66

3 Vertical structure of variability $\quad 69$

3.1 ECCO2 model description and processing . . . . . . . . . . 71

3.2 Current meter record processing . . . . . . . . . . . . . . . . . . . 72

3.3 ECCO2 model evaluation . . . . . . . . . . . . . 73

3.4 Vertical structure of currents in ECCO2 model . . . . . . . . . . . 85

3.5 Conclusions ........................... 94

4 Dynamics of low-frequency variability $\quad 97$

4.1 Existing theories . . . . . . . . . . . . . . . . 98 
4.2 Small-wavelength topography . . . . . . . . . . . . . . . . . . 103

4.3 Numerical solutions . . . . . . . . . . . . . . . . . . . . . . . . . . . 108

4.4 Global results . . . . . . . . . . . . . . . . . . 115

4.5 Effect of stratification and topography amplitude . . . . . . . . . . 118

4.6 Conclusions . . . . . . . . . . . . . . . . . 120

5 Model of the spectrum $\quad 125$

5.1 Dynamic model for low-frequency motion . . . . . . . . . . . . . 126

5.2 Spatially varying $4 \mathrm{D}$ spectrum of streamfunction . . . . . . . . . . 129

5.3 Comparison with observations . . . . . . . . . . . . . . 138

5.4 Applications . . . . . . . . . . . . . . . . . . 146

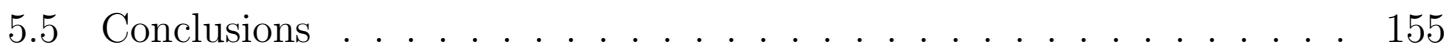

6 Conclusions $\quad 161$

6.1 Summary . . . . . . . . . . . . . . . . . . . 162

6.2 Future directions . . . . . . . . . . . . . . . 166

$\begin{array}{ll}\text { A Numerical methods } & 169\end{array}$

$\begin{array}{ll}\text { Bibliography } & 175\end{array}$ 


\section{List of Figures}

1-1 Two-dimensional zonal wavenumber-frequency spectrum for the region near $30^{\circ} \mathrm{N}, 190^{\circ} \mathrm{E}$, with westward propagation strongly dominant. Dispersion curves for basic theory barotropic (blue) and baroclinic (black) Rossby waves with no mean flow are shown with $l=0$ (dashed) and $l=k$ (dotted). The solid black line shows the dominant phase speed as found in from a Radon transform of the longitude-time plot. See Ch. 2 for details. . . . . . . . . . . . . . . . .

2-1 The along-track wavenumber spectrum from T/P (solid) and from the AVISO gridded product interpolated along the same track (dashed). Due to the smoothing imposed in the objective mapping procedure, the AVISO product has significantly lower energy at wavelengths shorter than $225 \mathrm{~km}$. Both spectra are along T/P arc 008 (equatorial crossing longitude $180^{\circ}$ ) between $30^{\circ} \mathrm{N}$ and $40^{\circ} \mathrm{N}$. Power laws are shown for reference. . . . . . . . . . . . . . . . .

2-2 The frequency spectrum from T/P (solid) and from the AVISO gridded product (dashed) at $35^{\circ} \mathrm{N}, 165^{\circ} \mathrm{E}$. Due to the smoothing imposed in the objective mapping procedure, the AVISO product has significantly lower energy at periods shorter than 55 days. . . . . . . . . . . . 
2-3 The time required for a long baroclinic Rossby wave to cross a basin $100^{\circ}$ wide (solid), roughly the width of the Pacific. The 18 year altimetric record (dashed) used in this study is sufficient to record Rossby waves crossing the Pacific equatorward of $35^{\circ}$. Basin transit times are calculated based on the basic theory for free waves in a flat-bottom, resting ocean [Chelton et al., 1998]. . . . . . . . . . . . . 43

2-4 Eddy kinetic energy, calculated from AVISO altimetry, varies by over four orders of magnitude over the ocean. . . . . . . . . . . . . .

2-5 Snapshot of SSH map used to compute spectra (top left). The longitudetime plot (right) shows the dominance of westward propagation of SSH anomalies, while the latitude-time plot (bottom left) does not show a clear pattern of meridional propagation. . . . . . . . . . . .

2-6 The Radon transform of the longitude-time plot of SSH (colored) is the projection $p(s, \theta)$ of the signal onto a line $L$ at angle $\theta$ relative to the longitude axis. The variance of $p(s, \theta)$ maximized when the line $L$ is perpendicular to the ridges and crests of the longitude-time plot, indicating the dominant phase speed. . . . . . . . . . . . . . . 46

2-7 The three-dimensional wavenumber-frequency spectrum for the region near $30^{\circ} \mathrm{N}, 190^{\circ} \mathrm{E}$. Slices through the planes $w=0, l=0, k=0$, and $k=6.6 \times 10^{-3} \mathrm{cpk}$ are shown. The red surface of constant power illustrates the dominance of westward propagation. For reference, the mesh surface shows the dispersion relation for classical theory of baroclinic Rossby waves. The black bar on the colorscale indicates a $95 \%$ confidence interval.

2-8 Two-dimensional zonal wavenumber-frequency spectrum for the region near $30^{\circ} \mathrm{N}, 190^{\circ} \mathrm{E}$. Dispersion curves for basic theory barotropic (blue) and baroclinic (black) Rossby waves with no mean flow are shown with $l=0$ (dashed) and $l=k$ (dotted). The solid black line shows the dominant phase speed as found from a Radon transform of the longitude-time plot. . . . . . . . . . . . . . 
2-9 Two-dimensional meridional wavenumber-frequency spectrum for the region near $30^{\circ} \mathrm{N}, 190^{\circ} \mathrm{E} . \quad \ldots \ldots \ldots \ldots$

2-10 Two-dimensional zonal and meridional wavenumber spectrum for the region near $30^{\circ} \mathrm{N}, 190^{\circ} \mathrm{E}$. Westward propagation is clearly dominant, and there is a slight excess of southward propagation. . . . . . .

2-11 One-dimensional zonal wavenumber (left) meridional wavenumber (center) and frequency (right) spectra for the region near $30^{\circ} \mathrm{N}, 190^{\circ} \mathrm{E}$. For zonal wavenumber, westward propagation (dashed) is stronger than eastward (solid) over most of the ocean. For meridional wavenumber, southward propagation (dashed) is stronger than northward (solid) in this region, but the imbalance is relatively small and regionally variable. The frequency spectrum is further divided into eastward (dash), westward (dash-dotted), and standing (dotted) components based on zonal wavenumber. Red lines show power laws with the indicated slope. 51

2-12 The 18-year mean SSH (1992-2010) still shows significant small-wavelength features corresponding to the high wavenumber, low frequency part of

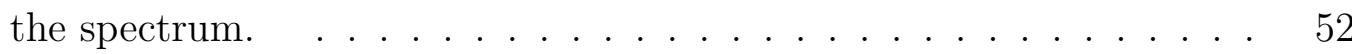

2-13 Two-dimensional wavenumber spectra of zonal (left) and meridional (right) velocity for the region near $30^{\circ} \mathrm{N}, 190^{\circ} \mathrm{E}$. Westward propagation dominates for both velocity components, but the spectra are approximately symmetric in the meridional direction. . . . . . .

2-14 Two-dimensional wavenumber spectra of time-mean zonal (left) and meridional (right) velocity for the region near $30^{\circ} \mathrm{N}, 190^{\circ} \mathrm{E}$ shows the signature of zonal striations. Westward propagation dominates for both velocity components, but the spectra are approximately symmetric in the meridional direction. While the spectra in Fig. 2-13 were integrated over all frequencies, here we show the spectra for $\omega^{-1}=18$ years. $\quad 55$ 
2-15 Two-dimensional zonal and meridional wavenumber spectrum of EKE for the region near $30^{\circ} \mathrm{N}, 190^{\circ} \mathrm{E}$. White ' + ' mark the peak, in $k$, of the spectrum at selected meridional wavenumbers, tracing a ridge of high

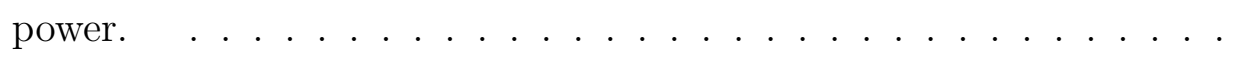

2-16 Two-dimensional zonal wavenumber-frequency spectrum for the region indicated at the top of each panel. The left panels show the North Pacific; the center panels shows the South Atlantic; the right panels show Indian Ocean, Kurohsio, Gulf Stream, and equatorial regions. The dominant phase speed is westward in all regions and increases toward the equator. . . . . . . . . . . . . .

2-17 Dominant zonal phase speed as found in from a Radon transform of the longitude-time plot (top left). Dominant period (top right), zonal wavelength (bottom left), and meridional wavelength (bottom right) based on the inverse of the first moment of the one-dimensional spectra. For zonal wavelength, the hatched area indicates regions where eastward propagation dominates; elsewhere, westward propagation dominates. For meridional wavelength, the hatched area indicates regions where northward propagation dominates; elsewhere, southward propagation dominates. . . . . . . . . . . . . . . .

2-18 The nonlinearity parameter $U / c$ is small near the equator, but increases with latitude. The black contour shows $U / c=1$. . . . . . . . . . .

2-19 Nonlinearity as a function of $k$ and $\omega$ based on the ratio of the turnover time $T(2.16)$ to wave period $\omega^{-1}$ at $30^{\circ} \mathrm{N}, 190^{\circ} \mathrm{E}$ (top). The black contour shows $T \omega=1$. The kinetic energy spectrum is shown with $T \omega=1$ contour superimposed, and most of the kinetic energy is at frequencies where nonlinearity is important (bottom). . . . . . . .

2-20 Same as Fig. $2-20$ but at $10^{\circ} \mathrm{N}, 190^{\circ} \mathrm{E}$ (top). The kinetic energy spectrum with $T \omega=1$ contour superimposed shows that much of the kinetic energy at this latitude extends to frequencies where nonlinearity is not significant (bottom). . . . . . . . . . . . . 
3-1 Locations of the 1068 mooring sites with records longer than 180 days and in water deeper than $3000 \mathrm{~m}$. . . . . . . . . . . . .

3-2 Global-mean total (solid) and eddy (dashed) kinetic energy (top), SSH, salinity, and potential temperature for the ECCO2 model. An imbalance in the un-optimized rain field imposed after 2002 results in a drop in SSH and rise in salinity. Pre-2002 salinity variations are very small; less than $0.001 \%$. Annual temperature variations are about $0.01^{\circ} \mathrm{C}$ with a warming trend of $0.004^{\circ} \mathrm{C} /$ year. . . . . . . . . . . . . 75

3-3 Mean dynamic topography (left) and SSH variance (right) from AVISO (top) and ECCO2 model (middle). Bottom row shows AVISO MDT minus ECCO2 MDT (left) and AVISO SSH variance minus ECCO2 $\mathrm{SSH}$ variance (right). For AVISO SSH variance, no value is shown if there are gaps in the record due to ice. Both fields have statistically significant spatial correlation between altimetric and model maps. The largest differences are in the position and sharpness of the major currents. See text for further discussion. . . . . . . . . . . . . .

3-4 Comparison of zonal wavenumber-frequency spectra of SSH from a low energy region $\left(25^{\circ}-30^{\circ} \mathrm{N}, 190^{\circ}-230^{\circ} \mathrm{E}\right.$; left $)$ and high energy region $\left(38^{\circ}-45^{\circ} \mathrm{N}, 150^{\circ}-165^{\circ} \mathrm{E}\right.$; right) in observations and ECCO2. Spectra from AVISO are shown (top), along with similar spectra from ECCO2 (middle), and the ratio of AVISO to ECCO2 power (bottom). Note that the ratio is plotted with a logarithmic colorscale, and the black contour shows a ratio of unity. . . . . . . . . . . .

3-5 Vertical profiles of $\overline{T K E}$ averaged over all moorings (solid), over all mooring locations in ECCO2 (dashed), and over the full ECCO2 domain (dotted). The number of moorings in each depth bin (open circles) is $10^{5}$ times the number shown. . . . . . . . . . . . . 
3-6 $\overline{T K E}(z)$ profiles as in Fig. 3-5, but divided into high energy regions (top) and low energy regions (bottom). $\overline{T K E}(z)$ profiles averaged over the full model domain are shown for reference (dotted line). Maps show the mooring locations used for each profile (right). . . . . . . . . 83

3-7 Vertical profiles of ECCO2 $\overline{T K E}$ normalized by CMD $\overline{T K E}$ for high energy region (dashed) and low energy region (dotted) as in Fig. 3-6. In the high energy region, the model $\overline{T K E}$ is about $83 \%$ of the CMD $\overline{T K E}$ averaged over the water column. In the low energy region, the average ratio is $45 \% \ldots \ldots \ldots \ldots \ldots$

3-8 Barotropic and first four baroclinic horizontal velocity modes $F_{n}(z)$ near $35^{\circ} \mathrm{N}, 220^{\circ} \mathrm{E}$ (left) based on ECCO2 model potential density profile (center) and buoyancy frequency (right) at that location. . . .

3-9 Vertical profile of $\overline{T K E}$ near $35^{\circ} \mathrm{N}, 220^{\circ} \mathrm{E}$ (solid) and reconstructed from the barotropic and first four baroclinic modes assuming no modal coupling (dotted) and with modal coupling (dashed). Without mode coupling, the modes cannot reconstruct the model $\overline{T K E}$ profile. . . .

3-10 Longitude-time plots of barotropic mode coefficient (left) and first baroclinic mode coefficient (right) for zonal velocity show that features propagate together in the two modes, suggesting that the modes are coupled. The section shown here is at $35^{\circ} \mathrm{N}$ spanning $180^{\circ}-220^{\circ} \mathrm{E}$

3-11 Temporal coherence (top) and phase (bottom) between the barotropic and first baroclinic mode coefficients near $35^{\circ} \mathrm{N}, 220^{\circ} \mathrm{E}$. The modes are coherent over all frequencies, and in phase such that they reinforce near the surface and cancel at depth. The $95 \%$ level of no significance is shown for coherence amplitude (dashed). . . . . . . . . . . . . . 90

3-12 Correlation between the barotropic and first baroclinic mode coefficients is positive almost everywhere in the ocean. Regions where correlation is not significant at the $99 \%$ confidence level are indicated by

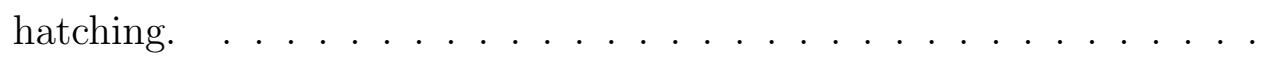


3-13 Four leading EOFs for $u$ near $35^{\circ} \mathrm{N}, 220^{\circ} \mathrm{E}$. These four EOFs account for $99 \%$ of the variance. The vertical structure of the first EOF is similar to the typical first baroclinic mode shape (Fig. 3-8), but offset so that it approaches zero at the bottom. . . . . . . . . .

3-14 As Fig. 3-9, but using the first five EOFs. In contrast to the classic Rossby wave modes, the EOFs reconstruct the model $\overline{T K E}$ profile without accounting for mode coupling. . . . . . . . . . . .

4-1 Large-wavelength Rossby waves can interact with rough topography. (a) A wave-like displacement over corrugated topography leads to a change in relative vorticity, and flow along the ridges and troughs. (b) The resulting convergence/divergence at crests/troughs drives flow downslope at one node of the initial displacement, and upslope at the other. The up/downslope flow generates relative vorticity at nodes of the initial displacement. (c) The sense of the relative vorticity tends to damp the initial displacement. Wavy lines represent large-wavelength wave displacement; solid arrows represent induced flow; dashed arrows represent induced relative vorticity. Adapted from Rhines and Bretherton [1973] Fig. 2. . . . . . . . . . . . . . . . . . 104

4-2 Typical area of bathymetric relief from the ECCO2 model. . . . . . 109

4-3 Flat bottom modes near $26^{\circ} \mathrm{N}, 150^{\circ} \mathrm{W}$ (left), potential density (middle), and buoyancy frequency (right). The barotropic (dashed), first baroclinic (solid), and higher baroclinic modes (grey) are shown. . . 110

4-4 The solutions $P_{n}^{m}(y, z)$ of (4.4) are a set of vertical and meridional modes. Shown are the pseudo-barotropic mode (top left), gravest baroclinic mode with no meridional nodes (top right), pseudo-barotropic mode with two meridional nodes (bottom left), and the gravest baroclinic mode with two meridional nodes (bottom right) near $26^{\circ} \mathrm{N}, 150^{\circ} \mathrm{W}$ for $k=-4 \times 10^{-4} \mathrm{cpk}$. The black line is the topography profile. . . . 
4-5 The behavior of a given solution to (4.4) (identified by its eigenvalue rank) evolves as wavenumber changes. At $k=-6.5 \times 10^{-3} \mathrm{cpk}$ (top) the $5^{\text {th }}$ solution (left) is bottom-trapped and the $9^{\text {th }}$ solution (right) meets the baroclinic mode criteria. At $k=-2 \times 10^{-3}$ cpk (middle), both solutions meet the baroclinic mode criteria. By $k=-6.5 \times$ $10^{-5} \mathrm{cpk}$, the $5^{\text {th }}$ solution meets the baroclinic mode criteria, while the $9^{\text {th }}$ solution has become bottom trapped. The evolution of each solution occurs smoothly, but leads to a kink in the dispersion curve for the gravest baroclinic mode. . . . . . . . . . . . . . .

4-6 The first 16 mean modes $P_{n}^{m}(z)$ over topography near $26^{\circ} \mathrm{N}, 150^{\circ} \mathrm{W}$. Pseudo-barotropic mode (dashed), gravest baroclinic (solid), and higher baroclinic modes (grey) are shown. The baroclinic mode is diminished at depth relative to the flat bottom first baroclinic mode. . . . . . .

4-7 The dispersion relation for the gravest baroclinic mode with topography (solid) shows a higher phase speed at all wavelengths than the first baroclinic modes without topography (dashed) and is in close agreement with the dominant phase speed from the SSH spectrum (colored). Near the kink in the dispersion curve, two solutions exist with similar large-scale structure but different small-scale structure. The kink occurs when the criteria described in the text change from the $5^{\text {th }}$ solution to the $9^{\text {th }}$ (thin dotted lines). These solutions are shown in Fig. 4-5. Also shown is the barotropic mode without topography (dashed). The dispersion relations are calculated near $26^{\circ} \mathrm{N}, 150^{\circ} \mathrm{W}$ and the spectrum is averaged over $25^{\circ}-30^{\circ} \mathrm{N}, 130^{\circ}-170^{\circ} \mathrm{W} \ldots \ldots$

4-8 Predicted zonal phase speeds for long Rossby waves are everywhere higher with topography (left) than in a flat bottom ocean (right). Figure $4-9$ shows the ratio. . . . . . . . . . . . . . . 116 
4-9 The ratio of the zonal phase speed for long Rossby waves with and without topography increases from $\sim 1$ near the equator to $\sim 2.5$ at $50^{\circ}$. The areas with phase speed enhancement greater than a factor of 3 are not relevant to observations, as described in the text. . . . . . . .

4-10 Zonal average zonal phase speeds (left) with topography (blue), in a flat bottom ocean (green), and the observations from Chelton and Schlax [1996] (crosses). The ratio of the predicted phase speeds with topography to the phase speeds with a flat bottom agree well with observations (right) at mid-latitudes, but observed phase speeds have a stronger latitude dependence than the prediction. . . . . . . . .

4-11 Map of bathymetry (left) and RMS topography height (right) used in the calculation of theoretical phase speed. . . . . . . . . . .

4-12 The phase speed increase with rough topography, $c_{\text {topo }} / c_{\text {flat }}$, depends on both the stratification and the topography amplitude (black lines, left axis). Lines show phase speed increase as a function of topography amplitude. Stratification is based on the OCCA climatology at $190^{\circ} \mathrm{E}$ and the latitude indicated. Also shown is a histogram of RMS topography height from Fig. 4-11 (grey bars, right axis). . . . . . . .

4-13 Zonal-mean buoyancy frequency from OCCA. Thermocline stratification is strongest at mid-latitudes, and weaker at the equator and high latitudes. This variation in stratification with latitude controls the degree of phase speed enhancement in the rough topography theory. 
5-1 The model spectrum (5.21) for SSH near $30^{\circ} \mathrm{N}, 190^{\circ} \mathrm{E}$. Marginal spectra $\Phi_{\eta}(k, \omega)$ (top left), $\Phi_{\eta}(l, \omega)$ (top right), and $\Phi_{\eta}(l, k)$ (bottom) are shown. Solid lines indicate the parameters in the model. One dimensional spectra are projected on a vertical plane, with high frequency/wavenumber power laws labeled. The ridge of westward variance is most prominent in the $k-\omega$ spectrum. Due to the difficulty of plotting positive and negative wavenumbers together on a logarithmic scale, we show the average of the positive and negative wavenumber half-spaces. . . . . . . . . . . . . . . . .

5-2 Same as Fig. 2-7, but for the model spectrum (5.21) for SSH near $30^{\circ} \mathrm{N}$,

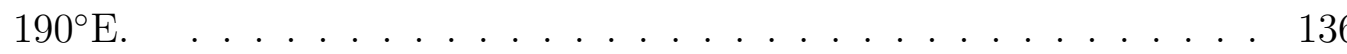

5-3 The zonal wavenumber-frequency spectrum of SSH near $30^{\circ} \mathrm{N}, 190^{\circ} \mathrm{E}$ from altimetry (left) and the model spectrum (right). . . . . . . 136

5-4 Global characteristics of the model spectrum (5.21). Dominant zonal phase speed estimated from the peak of the $k-\omega$ spectrum (top left), dominant period (top right), zonal wavelength (bottom left), and meridional wavelength (bottom right) based on the first moment of the associated one-dimensional spectra. Compare to Fig. 2-17. . . . . . . .

5-5 Along-track wavenumber (left) and frequency (right) spectra from tracks spanning $30^{\circ} \mathrm{N}$ to $40^{\circ} \mathrm{N}$ at the indicated longitude (solid line). Dashed line shows the model spectrum at each location. Vertical bars indicate the $95 \%$ confidence interval. . . . . . . . . . . . . . . .

5-6 Observed (solid) and modeled (dashed) frequency spectra of kinetic energy from moored instruments at $33^{\circ} \mathrm{N}, 232^{\circ} \mathrm{E}$. Instrument depths are $77 \mathrm{~m}$ (top left), $431 \mathrm{~m}$ (top right), $731 \mathrm{~m}$ (bottom left), and $1481 \mathrm{~m}$ (bottom right). . . . . . . . . . . . . . . .

5-7 Observed (solid) and modeled (dashed) frequency spectra of kinetic energy from moored instruments at $33^{\circ} \mathrm{N}, 232^{\circ} \mathrm{E}, 1481 \mathrm{~m}$ deep, as in Fig. 5-6, but now expanded to show the internal wave regime. The spectral peaks are at the inertial and $M_{2}$ tidal frequencies. . . . . . 142 
5-8 Observed (solid) and modeled (dashed) frequency spectra of potential temperature from moored current meters at $33^{\circ} \mathrm{N}, 232^{\circ} \mathrm{E}$. Instrument depths are $77 \mathrm{~m}$ (top left), $431 \mathrm{~m}$ (top right), $718 \mathrm{~m}$ (bottom left), and $1481 \mathrm{~m}$ (bottom right). . . . . . . . . . . . . . . .

5-9 Observed (solid) and modeled (dashed) wavenumber spectra of kinetic energy from shipboard ADCP. The transect spans $20^{\circ}-30^{\circ} \mathrm{N}$ at $180^{\circ} \mathrm{E}$ at $100 \mathrm{~m}$ depth. The high wavenumber spectral slope for the model is $k^{-2}$

5-10 Autocorrelation function for temperature as a function of zonal separation (top left), meridional separation (top right), and temporal separation (bottom left) based on the model spectrum at $30^{\circ} \mathrm{N}, 190^{\circ} \mathrm{E}$. From the three-dimensional model spectrum (5.21), we can compute the spatial correlation at a particular frequency, $\operatorname{Cor}\left(r_{x} ; \omega\right)$ by $(5.42)$ (bottom right). The zonal correlation is slightly larger at low frequency (1/20 years $^{-1}$; solid) than high frequency $\left(1 / 15\right.$ day $^{-1}$; dashed $) . .$.

5-11 Autocorrelation function for zonal (solid) and meridional (dashed) velocity as a function of zonal separation (left), meridional separation (right), based on the model spectrum at $30^{\circ} \mathrm{N}, 190^{\circ} \mathrm{E} . \quad$. . . . . . 150

5-12 Spatial correlation function for SSH used in the AVISO data mapping (left) and based on the model spectrum (5.21) (right) at lags of 0 days (top), 20 days (middle), and 40 days (bottom) at $30^{\circ} \mathrm{N}, 320^{\circ} \mathrm{E}$ in the North Atlantic. Contour interval is 0.1. . . . . . . . . . . . . . . 156

5-13 Zonal-temporal correlation function for SSH used in the AVISO data mapping (left) and based on the model spectrum (5.21) (right) at $30^{\circ} \mathrm{N}$, $320^{\circ} \mathrm{E}$ in the North Atlantic. Contour interval is 0.1. . . . . . . . . 156

A-1 The grid used to discretize (A.1) . . . . . . . . . . . . . . 170 


\section{Chapter 1}

\section{Introduction}

This work is motivated by the recognition that a comprehensive description of ocean variability is as important to our understanding of ocean circulation as a detailed model of any particular process contributing to that variability. Detailed process studies are typically restricted to a particular range of scales and consider other variability to be troublesome noise. However, no process can be be fully understood without the broader context of other scales of ever-present ocean variability. This thesis is concerned with identifying the broad patterns, rather than the analysis of specific events.

In a numerical modeling context, a baseline description of oceanic variability is useful as a test of ocean General Circulation Models (GCMs). An accurate GCM should not only reproduce specific features of the observed ocean, such as the separation point of the Gulf Stream or the mean velocity of the Antarctic Circumpolar Current, but also the variability in these quantities.

In an observational context, a description of the baseline level of oceanic variability is crucial for estimating the significance of apparent trends in ocean circulation and for observing system design. The prospect of climate change and associated changes in ocean circulation bring the need to monitor the mean circulation. However, such efforts are hampered by our incomplete understanding of the space and time scales of ocean variability. In particular, inferences about the significance of observed trends depend on the spectrum of the background "noise" or stochastic variability. The low- 
frequency variability described in this thesis constitutes the noise obscuring possible trends in ocean circulation.

The time required to detect a secular trend depends on the spectrum of the background noise. As a concrete example, Wunsch [2008] used existing knowledge of the frequency and wavenumber spectral content of geostrophic eddies to show that observations of meridional transport will exhibit multi-year variations even in the absence of any secular trend. In general, a frequency spectrum that is "red" at low frequency implies that random fluctuations decorrelate very slowly, making trend detection difficult. But the full noise spectrum determines whether it is more efficient to make continuous measurements over a short duration (as with moorings) or discrete measurements over a longer interval (as with repeat hydrographic sections). In short, knowledge of the full frequency and wavenumber content of the noise is necessary for determining the optimal space and time sampling requirements for trend detection.

One typical approach for observing system feasibility studies involves simulating the system within a GCM [Hirschi et al., 2003; Baehr et al., 2008a]. Simulated "data" are extracted from a GCM run and analyzed in the same way that the real data will be. Results from the simulated data can then be compared to the "correct" answer, calculated from the full GCM output, and the observing system can be adjusted accordingly. Adjustments to the observing system rely largely on physical intuition, and this method has been called "heuristic array design" [Baehr et al., 2008b]. This approach is limited by the fact that GCMs may not accurately represent the dynamics of interest and, even if the model does, it is always with a significantly limited number of degrees of freedom. Such studies usually do not have realistic noise elements, often neglecting instrument noise and some dynamical processes such as internal waves.

Other approaches to observing system design exist. One approach involves using an adjoint model to estimate the sensitivity of the quantity of interest, such as meridional heat or volume transport, to other elements of the ocean state [Heimbach et al., 2011]. In this method, the sensitivity of the meridional heat transport, or any other aspect of the ocean circulation, to the whole ocean state can inform observing system design. Various schemes for optimizing oceanographic experimental design have been 
described for relatively simple experiments [Barth and Wunsch, 1990; Barth, 1992]. Baehr et al. [2008b] describe both sequential and global optimization of an observing system deployed to monitor the North Atlantic meridional overturning circulation. In general, such optimization techniques are directed at maximizing the signal-to-noise ratio for the quantity of interest, and doing this requires knowing the frequency and wavenumber content of the background noise. Describing this noise is a major goal of this thesis.

A number of approaches exist for quantifying the baseline oceanic variability. For example, maps of mean/eddy kinetic energy and variance of other quantities (velocity, density, temperature, salinity, etc.) provide a useful starting point. But such maps do not quantify the spatial or temporal scales present, and they are not easily related to other contexts, such as ocean turbulence theory.

The most natural approach to quantifying the baseline of oceanic variability is to estimate the multi-dimensional spectrum of oceanic variability for different quantities. Multi-dimensional spectra quantify the level of variability at different space and time scales, and contain information not accessible from a set of one-dimensional spectra. At the same time, multi-dimensional spectra are easily reducible to one-dimensional wavenumber spectra, which are easier to interpret. Also, the power spectrum is related, via the Fourier transform, to the autocorrelation function which can be used for uncertainty estimates.

The primary goal of this thesis is to create a model of the 4-dimensional spectrum (frequency, zonal-, meridional-, and vertical-wavenumber) that usefully summarizes the oceanic variability for a typical ocean region. A similar project was pioneered by Zang [2000] and Zang and Wunsch [2001] who found that a useful spectral description does exist, and provided a framework for relating spectra of pressure, velocity, density, and vertical displacement for geostrophic motions.

The model presented by Zang [2000] is quasi-universal, in the sense that it was only intended to apply in the ocean interior (away from boundary currents, the equator, the Antarctic Circumpolar Current (ACC), and very high latitudes) and has a fixed shape but variable amplitude. The model spectrum also has a separable form. Wunsch 
[2010] proposed an updated model of the frequency-wavenumber spectrum which attempts to incorporate the most conspicuous anisotropy in the observed spectrum. We seek to refine these models with the benefit of a longer data record and extending the description to incorporate meridional structure. We will also describe the global variation in the shape of the spectrum.

The model proposed here for low-frequency motions will have a number of uses, including estimating the significance of apparent trends, aiding the design of observational studies, testing models, identifying anomalous regions, and in identifying gaps in our understanding of ocean dynamics.

This project is inspired by the internal wave spectrum proposed by Garrett and Munk $[1972,1975]$. They were able to patch together disparate observations to form a more complete description of internal waves, which has become known as the GarrettMunk spectrum. Though only intended to be provisional, the Garrett-Munk spectrum served as a useful focal point for many internal wave studies. The assertion of a universal spectrum posed a puzzle to be explained or refuted and allowed for the identification of unusual regions. Their work made it possible to remove the internal wave "noise" when the mean or slowly varying ocean state was of interest. Finally, the Garrett-Munk spectrum was used for basic estimates of the mixing generated by internal waves, advancing our understanding of their role in ocean dynamics.

There are reasons to expect a universal spectrum for internal waves. Internal waves are forced at large scales, and the Garrett-Munk spectrum is the result of weakly interacting waves [Lvov et al., 2004]. However, the situation is not so clear for geostrophic motions. The mean flow and beta-effect (the variation in the Coriolis parameter with latitude) play a strong role in geostrophic dynamics, and both vary widely over the ocean, likely leading to different dynamical regimes and spectral shapes throughout the ocean. Even if no truly universal spectrum exists for geostrophic motions, a description of the average spectrum will have value as a tool for identifying regions inconsistent with the base state model. Some theoretical predictions for the shape of the spectrum of geostrophic motions are discussed below. 


\section{$1.1 \quad$ Theoretical background}

There is a large and often conflicting literature related to the spectrum of lowfrequency oceanic variability. One set of theories predicts the vertical structure of geostrophic motions under different conditions [Philander, 1978; Killworth and Blundell, 2001; Lapeyre and Klein, 2006; LaCasce, 2012; Smith and Vanneste, 2012; Scott and Furnival, 2012]. Another body of literature predicts wavenumber and, less often, frequency spectral slopes resulting from turbulent cascades or due to atmospheric forcing [Blumen, 1978; Charney, 1971; Müller and Frankignoul, 1981; Held et al., 1995; Killworth and Blundell, 2007]. A third body of literature attempts to explain the observed phase speed of propagating sea surface height features in terms of linear [Keller and Veronis, 1969; Rhines, 1970; Rhines and Bretherton, 1973; Samelson, 1992; Killworth et al., 1997; Dewar, 1998; de Szoeke and Chelton, 1999; Killworth and Blundell, 1999, 2004, 2005; Reznik and Tsybaneva, 1999; Bobrovich and Reznik, 1999; Tailleux and McWilliams, 2001; Maharaj et al., 2007; Kubokawa, 2008; Aoki et al., 2009; Durland et al., 2011] or nonlinear [McWilliams and Flierl, 1979; Vanneste, 2003; LaCasce and Pedlosky, 2004; Early et al., 2011] Rossby waves. Finally, the role of baroclinic instability in setting the observed eddy wavelengths has also been considered. We briefly review what we regard as the more relevant theories below, beginning with the vertical structure [Smith, 2007; Tulloch et al., 2009].

Satellite altimetry is exceptional in oceanography for its ability to give nearly global, continuous coverage in both space and time. Given the periods and wavelengths observed by altimetry (after removing the tidal signal), sea surface height observations are typically interpreted in terms of quasi-geostrophic $(\mathrm{QG})$ dynamics, described by the potential vorticity $(\mathrm{PV})$

$$
q=f_{0}+\beta y+\nabla^{2} \psi+\frac{\partial}{\partial z}\left(\frac{f_{0}^{2}}{N^{2}} \frac{\partial \psi}{\partial z}\right), \quad-H<z<0
$$


and buoyancy at the top and bottom boundaries

$$
b=\left.f_{0} \frac{\partial \psi}{\partial z}\right|_{z=0,-H}
$$

where $b=-g \rho / \rho_{0}$ is the buoyancy anomaly, $\rho$ is the density, $\rho_{0}$ is the reference density, $\psi$ is the geostrophic streamfunction, $f_{0}$ is the Coriolis parameter, $\beta$ is the planetary vorticity gradient, $N$ is the buoyancy frequency, and $H$ is the ocean depth. In the QG approximation, PV is advected by the horizontal geostrophic velocity $(u, v)=\left(-\psi_{y}, \psi_{x}\right)$

$$
\frac{\partial q}{\partial t}+J(\psi, q)=0, \quad-H<z<0
$$

where $J(a, b)=a_{x} b_{y}-a_{y} b_{x}$ is the Jacobian. Buoyancy is subject to both advection and forcing,

$$
\frac{\partial b}{\partial t}+J(\psi, b)=\mathcal{F}, \quad z=0,-H
$$

where $F$ includes any forcing and dissipation of buoyancy.

Equations (1.1) and (1.2) can be solved for the streamfunction $\psi$ if $q$ and $b$ are known, thereby determining the vertical structure of motions. The traditional approach assumes a flat bottom, rigid lid, resting mean state, and neglects the surface buoyancy anomaly. (We will refer to this as the basic theory.) In this case, the solutions are the classic free modes used by Wunsch [1997] to describe the vertical partition of kinetic energy. Extended theories include the interior potential vorticity due to the mean flow by linearizing around a non-resting state. An alternative scheme, known as surface quasi-geostrophy (SQG), accounts for the surface buoyancy anomalies but neglects the mean interior PV field [Blumen, 1978; Held et al., 1995; Lapeyre and Klein, 2006]. Scott and Furnival [2012] have proposed another representation that accounts for the sea surface height anomaly. The various solutions of (1.1) and (1.2), including the QG, extended QG, SQG, and the Scott and Furnival [2012] proposal, each provide different vertical basis functions for describing the vertical structure of variability. At present, it is not clear which of these methods provides the most efficient basis or which is most dynamically relevant. 
In addition to the vertical structure discussed above, QG and SQG theories predict the shape of the wavenumber spectrum of sea surface height. QG turbulence theory predicts a forward enstrophy cascade for wavenumbers larger than the energy injection scale $k_{I}$ [Charney, 1971], and an inverse energy cascade for smaller wavenumbers. The kinetic energy spectra in the forward and inverse ranges are

$$
\begin{array}{ccc}
E(k) \sim k^{-3} & \text { for } & |k|>k_{I} \\
E(k) \sim k^{-5 / 3} & \text { for } & |k|<k_{I}
\end{array}
$$

For geostrophic motions, this implies a $k^{-5}$ power law for SSH for $|k|>k_{I}$, and $k^{-11 / 3}$ for $|k|<k_{I}$. SQG turbulence theory predicts power laws of

$$
\begin{array}{rlrl}
E(k) & \sim k^{-5 / 3} & \text { for } & |k|>k_{I} \\
E(k) \sim k^{-1} & \text { for } & |k|<k_{I}
\end{array}
$$

in the forward and inverse ranges, respectively [Held et al., 1995]. The corresponding SSH spectral slopes are $k^{-11 / 3}$ for $|k|>k_{I}$ and $k^{-3}$ for $|k|<k_{I}$. The energy injection wavenumber appears to be close to the deformation scale [Scott and Wang, 2005]. Since the altimeter only barely resolves the deformation scale over most of the ocean and this thesis will primarily focus on the low wavenumber regime where the inverse cascade likely dominates, the low wavenumber spectral slopes are likely more relevant.

$X u$ and $F u$ [2011, 2012] have mapped the high-wavenumber spectral slope of sea surface height estimated from altimetry. They show that the spectral slope is everywhere flatter than the $k^{-5}$ predicted by QG theory. Over most of the ocean, the slope is flatter than $k^{-11 / 3}$, but approaches $k^{-4}$ in the high energy boundary current and ACC regions. While such observations call into question the assumptions of the SQG turbulence theory, the SQG dynamics may still be important in some regions.

Most turbulence theories predict wavenumber spectra. Ferrari and Wunsch [2010] consider the possibility that the frequency spectrum is the result of variability being swept past a fixed point in space (as in the "Taylor hypothesis") or of waves 
propagating zonally with phase speed $c_{x}$. In either case, the frequency spectrum would have the same spectral slope as the wavenumber spectrum: $\omega^{-3}$ if interior PV dynamics dominate, or $\omega^{-11 / 3}$ for high frequencies if surface buoyancy anomalies dominate. Observations suggest $\omega^{-2}$ in several areas, inconsistent with both QG and SQG turbulence predictions. This result is unsurprising given that the corresponding wavenumber spectral slopes are not observed either [Xu and Fu, 2012]. Arbic et al. [2012] have attempted to estimate turbulent spectral fluxes in the frequency domain from altimetry. They find that the Taylor hypothesis fails, consistent with Ferrari and Wunsch [2010].

The QG and SQG theories above describe the sea surface variability as the result of a turbulent cascade of potential vorticity and buoyancy variance from large wavelength to small wavelength. In contrast, Frankignoul and Müller [1979a,b] and Müller and Frankignoul [1981] considered the quasi-geostrophic response of the ocean to direct atmospheric forcing. Their model was a continuously stratified, $\beta$-plane ocean of constant depth and infinite horizontal extent, with all dissipation processes parameterized as linear scale-independent friction. (Rayleigh damping; lateral diffusion and bottom friction are also considered but dismissed.) They decomposed the response into the standard flat-bottom, resting ocean vertical eigenmodes, and discuss the barotropic and baroclinic response of the ocean to the integrated atmospheric forcing. They model the wind forcing as white in frequency and isotropic in wavenumber. While the observed wind stress spectrum is clearly frequency dependent, they argue that the spectrum is nearly white at the wavelengths smaller than $4000 \mathrm{~km}$ that they consider. Their most relevant finding is that direct atmospheric forcing can account for the level of variability observed in the low-energy parts of the ocean, i.e. away from boundary current regions [Müller and Frankignoul, 1981, see their Fig. 16].

The vertical structure of forced motions is similar to that of SQG theory, as we should anticipate since both assume motion is driven by surface PV anomalies. However, they differ in that Müller and Frankignoul [1981] consider a forced problem (with the vertical Ekman velocity being the dominant term), while SQG theory assumes buoyancy anomalies are generated at the energy injection scale $k_{I}$ spread through 
an turbulent inertial range. Further, SQG imposes the artificial constraint of zero interior PV.

Müller and Frankignoul [1981] present a detailed analysis of the frequency spectrum of the quasi-geostrophic oceanic response to atmospheric forcing. The predicted frequency spectrum of total baroclinic energy depends on whether the oceanic response is resonant or off-resonant, though the relevance of resonance to observations is not clear. The theoretical oceanic response hypothetically can be resonant at frequencies below $\omega_{n}^{\max }$, the maximum Rossby wave frequency for the $n^{\text {th }}$ vertical mode. For Rayleigh damping, the spectrum is white at low frequencies, changing smoothly to a $\omega^{-2}$ power law at $\omega \sim \omega_{n}^{\max }$ (their Fig. 7). The maximum frequency for the first baroclinic mode, $\omega_{1}^{\max }=\beta L_{d} / 2$, depends on latitude primarily through the deformation radius $L_{d}$. Thus, they predict that the break point between the white low-frequency spectrum and $\omega^{-2}$ power law will decrease with latitude as the deformation radius decreases.

For the wavenumber spectrum of total energy in the $n^{\text {th }}$ mode, Müller and Frankignoul [1981] predict

$$
E_{\text {tot }}^{n} \sim \frac{1}{k^{3}+k L_{d}^{-2}}
$$

For the baroclinic modes, the spectrum transitions from a $k^{-1}$ power law at low wavenumber to a steeper $k^{-3}$ at high wavenumber with the break point near the deformation radius. For the barotropic mode, the predicted spectrum is a $k^{-3}$ power law at high wavenumber.

Quasi-geostrophic theory also predicts planetary wave propagation characteristics. Whereas the energy spectra discussed above are the result of nonlinear interactions, both linear and nonlinear theories have been developed to explain observed wave propagation. The observation by Chelton and Schlax [1996] of sea surface height anomalies propagating faster than the long baroclinic Rossby wave phase speed inspired several theories extending the basic theory to explain the observations. The effects of mean flow and sloping topography have received much attention. Dewar [1998] used a layered QG model to show that baroclinic waves in the presence of a 
mean flow can be accelerated by the mean advective field as well as by the mean potential vorticity field. Killworth and Blundell [1999] showed that uniformly sloping topography can increase or decrease westward phase speeds, depending on the direction of the slope. Killworth and Blundell [2004, 2005] extended the work of Dewar [1998] to the continuously stratified case and also included sloping topography. These extended theories are evaluated by Maharaj et al. [2007], and appear to explain much of the observed phase speed anomaly. Meanwhile, it has been suggested that the observed features are not long Rossby waves after all, but rather nonlinear coherent vortices [Chelton et al., 2007; Early et al., 2011].

Other attempts to explain the propagation of sea surface height signals have focused on the role of small wavelength "rough" topography. These effects were considered by Rhines and Bretherton [1973] for a homogeneous ocean. They used the term "slope" for topographic wavelengths $L_{b}$ much larger than the wavelength of the motion $k^{-1}$, "undulations" for $L_{b} \sim k^{-1}$, and "roughness" for small wavelength variations $L_{b} \ll k^{-1}$. Reznik and Tsybaneva [1999] studied the effect of rough topography $^{1}$ on a two-layer ocean, and Bobrovich and Reznik [1999] extended the theory to constant stratification. Rough topography leads to consistently increased wave propagation speeds and alters the vertical structure of Rossby waves. A related hypothesis notes that bottom pressure variations are often uncorrelated with sea surface height variations, leading to similar effect on the vertical structure and propagations speed [Tailleux and McWilliams, 2001].

Attempts have also been made to interpret the propagating sea surface height signals in terms of planetary waves and geostrophic turbulence arising from baroclinic instability [Tulloch et al., 2009]. They abandoned the long-wave approximation, and instead choose a wavelength at each latitude that gives the observed dominant phase speed. The best-fitting wavelength decreases from about $600 \mathrm{~km}$ equatorward of $30^{\circ} \mathrm{N}$ to about $100 \mathrm{~km}$ at higher latitudes. The authors suggest that this change in wavelength is associated with a change from wavelike dynamics at low latitudes to

\footnotetext{
${ }^{1}$ The effect of rough topography on Rossby waves is related to form drag. The term "roughness" should not be taken to imply frictional effects.
} 
turbulent dynamics at high latitudes. However, this theory explains one speed and one wavelength, and the implications for the observed broad spectrum of variability are unclear.

\subsection{Previous work}

While there were numerous early attempts to describe the spectrum of ocean variability from moorings, most recent work is based on satellite altimetry. The satellite record of sea surface height is the longest global-coverage oceanographic dataset, currently including 19 years of data since the TOPEX/POSEIDON mission began providing high quality observations [Fu et al., 1994]. Combining data from the TOPEX/POSEIDON and Jason-1 and -2 satellites, which follow the same orbit, with data from ERS-1/2 and ENVISAT, which follow a different orbit, gives a higher resolution dataset than either altimeter would produce on its own [Le Traon et al., 1998]. The tradeoff is that the merged data product loses resolution in the along-track direction, and may produce artifacts or obscure real features of the original data. Wavelengths longer than $2^{\circ}$ appear to be well represented in the merged data product [Ducet et al., 2000; Chelton et al., 2011].

Early attempts to characterize mesoscale ocean variability include Stammer and Böning [1992], who used GEOSAT observations, and Stammer [1997] using TOPEX/POSEIDON altimeter measurements. Others have focused specifically on spectral descriptions of ocean variability [Wunsch and Stammer, 1997; Zang, 2000; Zang and Wunsch, 2001].

$F u[2009]$ estimated the zonal and meridional propagation speed of eddy variability based on cross-correlations of sea surface height time series, resulting in maps of eddy propagation speed. That study provides information complimentary to the multidimensional spectral analysis taken here. Zonal eddy velocities are primarily westward outside the tropics, but are clearly affected by mean currents and topography. In the tropics, meridional eddy velocities are convergent toward the equator in the western part of the basins and divergent away from the equator in the eastern part of the 
basins.

One limitation of the altimetric record (among many) is that it only represents the surface pressure distribution, with no information about the vertical structure. Moorings have been used to estimate the frequency distribution and vertical partition of kinetic energy, vertical displacement, and temperature variance. Schmitz [1978, 1988] characterized the vertical distribution of kinetic energy in the mid-latitude North Atlantic and Pacific. As a first approximation, the vertical profile of kinetic energy is independent of location, decreasing rapidly from the surface to about $1000 \mathrm{~m}$, and relatively constant below. However, the ratio of surface to abyssal kinetic energy was found to decrease near the Gulf Stream, i.e. with increasing kinetic energy. Wunsch [1997] further developed the description of the vertical partition of kinetic energy, showing that the barotropic and low baroclinic modes dominate.

Wunsch [1999] estimated the vertical displacement of isopycnals in the North Atlantic from moored temperature records. He showed that the first baroclinic mode accounts for 30\%-40\% of the potential energy away from the western boundary current. Forget and Wunsch [2007] estimated the full three dimensional distribution of temperature and salinity variability from historical hydrography. In the mid-latitudes away from western boundary currents and below $100 \mathrm{~m}$, temperature and salinity variability were consistent with vertical displacement of mean gradients.

Due to the observational difficulty, estimates of the wavenumber distribution of sub-surface motions are relatively rare. The Mid-Ocean Dynamics Experiment (MODE) allowed estimation of space and time correlation functions for velocity and temperature [Richman et al., 1977]. Wavenumber spectra of temperature have been estimated for the Pacific from XBT sections [Bernstein and White, 1977; Roemmich and Cornuelle, 1990]. Sections from the northwest Pacific (near the Kuroshio) show significantly higher energy in the $500 \mathrm{~km}-1000 \mathrm{~km}$ band than do central Pacific sections. In the central Pacific, wavenumber spectra are roughly consistent with direct wind forcing, but wind forcing is insufficient to explain the larger energy levels in the western part of the basin [Müller and Frankignoul, 1981]. 


\subsection{Outline}

Despite the efforts outlined above, we lack a comprehensive description of oceanic variability. Previous efforts to describe the oceanic variability spectrum lack an accurate representation of the geographical changes in the spectrum and of the vertical structure of variability. First, the quasi-universal isotropic spectrum proposed by Zang [2000] does not reflect the observed changes in dominant period and wavelength with location. Second, the vertical structure proposed does not reflect the apparent mode coupling [Wunsch, 1997], resulting in a vertical partition of kinetic energy that is unrealistically uniform in depth. These deficiencies limit its use for estimating the significance of trends or determining sampling requirements. This thesis is an attempt to address these issues.

Chapter 2 describes the three-dimensional wavenumber-frequency spectrum of sea surface height variability based on AVISO gridded satellite altimetry. Figure. 1-1 shows a typical altimetric zonal wavenumber-frequency spectrum. See Ch. 2 for details on the underlying data and analysis. The figure shows the presence of energy at all observed frequencies and wavenumbers, and this thesis is directed at describing and explaining this structure. The most prominent feature of the spectrum is the dominance of westward motions (negative zonal wavenumber $k$ ), which we call the "ridge of westward variance" [Chelton et al., 2007; Wunsch, 2010]2. Westward motion is expected due to the beta effect, but the observed motions do not follow the basic Rossby wave dispersion curves (dashed and dotted lines). Instead, the motions appear largely nondispersive, with a constant phase speed over a large range of wavelengths (solid line). This dominant phase speed changes with latitude and the mean flow, though the phase speed typically exceeds the basic theory prediction. The dominant periods and wavelengths, defined here as the inverse of the first moment of the spectrum, also change with location.

\footnotetext{
2 Wunsch [2010] used the term "nondispersive line" to describe the feature in $\omega$ - $k$ spectra. However, as we describe in Ch. 2, the ridge of dominant energy extends over a wide range of meridional wavenumbers, suggesting a more dispersive nature in that direction. Since we will focus on the three-dimensional spectrum, where the feature is not nondispersive, we will use the term "ridge of westward variance" instead.
} 


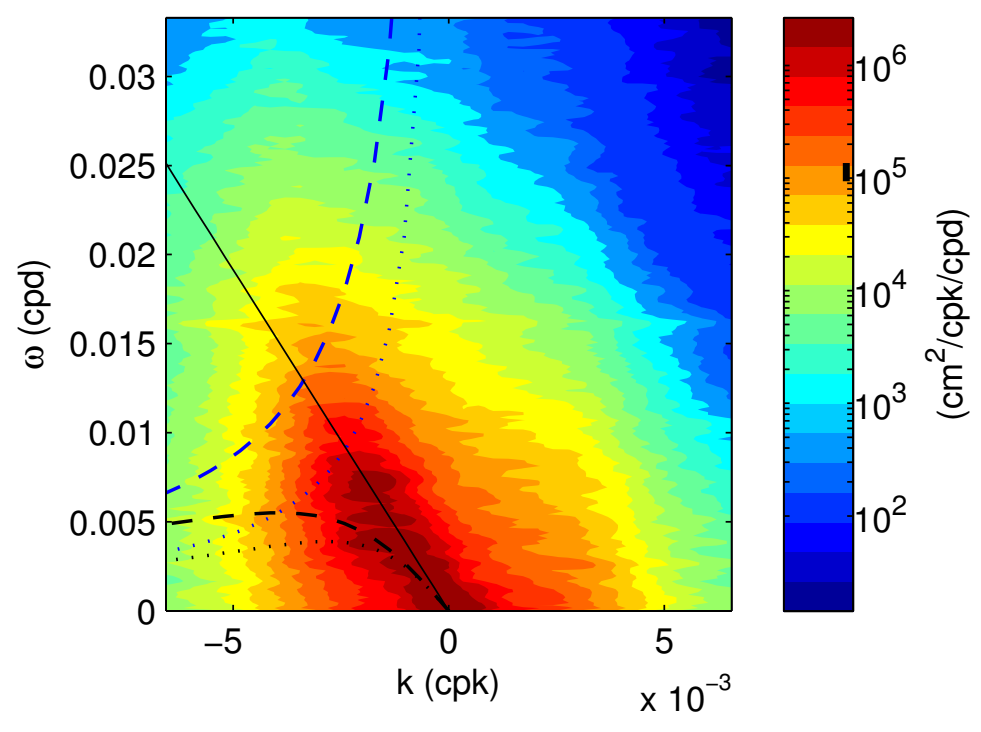

Figure 1-1: Two-dimensional zonal wavenumber-frequency spectrum for the region near $30^{\circ} \mathrm{N}, 190^{\circ} \mathrm{E}$, with westward propagation strongly dominant. Dispersion curves for basic theory barotropic (blue) and baroclinic (black) Rossby waves with no mean flow are shown with $l=0$ (dashed) and $l=k$ (dotted). The solid black line shows the dominant phase speed as found in from a Radon transform of the longitude-time plot. See Ch. 2 for details.

Chapter 3 describes the vertical structure of ocean variability. Moored current meters are used to estimate the partition of the surface variability into vertical modes, but the record is sparse and point measurements give no information about horizontal wavelength. Also, most current meter records are too short to definitively estimate the degree of coupling between modes, making it difficult to accurately describe the vertical structure in terms of simple dynamical modes. The ECCO2 model is used to supplement the observations. In the ECCO2 model, the basic Rossby wave modes are found to be strongly coupled, resulting in increased kinetic energy at the surface and reduced kinetic energy at depth relative to the case of uncoupled modes.

In Chapter 4 we develop a theory for the impact of rough topography on the propagation of long Rossby waves in an ocean with realistic stratification. Rough topography with an amplitude of order $10 \mathrm{~m}$ or more is found to increase the phase speed of Rossby waves and change the vertical structure. In particular, the resulting vertical structure is surface intensified and diminished at depth relative to the basic theory. Since the ocean bottom is everywhere rough by our definition, we propose 
that rough topography is plays an important role in setting the observed phase speed and vertical structure of geostrophic motions.

Chapter 5 presents an empirical model for the four-dimensional spectrum of oceanic variability, based on the observed spectrum of surface variability and information about the vertical structure of the variability from the ECCO2 model and current meters. The goal is to construct a useful quasi-universal spectral description of oceanic variability that is consistent with our dynamical understanding. The model is formulated in terms of an analytical function for the three dimensional structure (zonal- and meridional-wavenumber and frequency) with geographically varying parameters and a partition between the dynamical modes for Rossby waves over rough topography. We also illustrate the applications of this model spectrum for observing system design and for estimating the significance of observed trends.

\subsection{Questions raised}

As is often the case, our attempt to describe the spectrum of ocean variability raises at least as many questions as it answers. In particular:

- What sets the wavenumber spectral slope? Are the spectral slopes due to turbulent cascades (presumably with different dynamical regimes controlling the spectral slope)? Or are the energy injection and dissipation wavelengths too close together for a an inertial range with clear spectral slope to be observed?

- What sets the frequency spectral slope? Unlike wavenumber, the frequency spectral slope appears to be nearly constant at $\omega^{-2}$ for frequencies between $10^{-2}$ and $10^{-1}$ cpd.

- What role does atmospheric forcing play in shaping the observed spectrum?

- To what extent are the observed motions coherent nonlinear vortices as opposed to linear waves?

- What is the group velocity of wave-like components? 
We hope that the model spectrum will serve to focus future efforts on answering these questions. 


\section{Chapter 2}

\section{Observed SSH $k, l, \omega$ spectrum}

Altimetry provides our only continuous, near-global record of ocean variability. As such, altimetry provides the nearest thing to a comprehensive description of ocean physics. Though it is limited to surface motion, geostrophic motions mainly have large vertical scale. Furthermore, SSH is closely related to other more dynamically relevant quantities such as the near surface velocity, kinetic energy, and pressure fields. Many altimetric studies focus on particular particular dynamical process such as eddy diffusivity near jets [Ferrari and Nikurashin, 2010], monitor changes in ocean circulation [Farrar and Weller, 2006], or look for evidence of turbulent energy cascades [Scott and Wang, 2005]. Yet a comprehensive description of the three-dimensional spectrum of ocean variability is lacking.

Wunsch and Stammer [1995] constructed a model for the global average frequencywavenumber spectrum in terms of spherical harmonics. Stammer [1997] studied regional frequency and wavenumber spectra and concluded, based on filtered along-track wavenumber spectra, that SSH spectra displayed a remarkably universal $k^{-4}$ power law at wavelengths shorter than $400 \mathrm{~km}$. The primary variation in the spectral shape was associated with kinetic energy level and in the cutoff wavenumber between the low-wavenumber plateau and high wavenumber $k^{-4}$ spectral slope. Xu and Fu [2012] criticized the filtering in Stammer [1997], and estimated wavenumber spectral slopes globally from along-track altimetry. Their results showed strong regional variation in spectral slope, from $k^{-4.5}$ in high energy regions to $k^{-2}$ in low energy regions, 
contradicting claims of a universal wavenumber spectrum.

Zang and Wunsch [2001] present an empirical model for the global average frequencywavenumber spectrum, and propose a vertical structure for variability based on Wunsch [1997]. Their model spectrum is universal and isotropic, neglecting many features of the observed spectrum. A major goal of this chapter is to provide a more nuanced look at the three-dimensional spectrum of sea surface height.

Much research has focused on one of the most conspicuous features of the altimetry: the widespread appearance of westward propagating features. Early studies found that sea surface height anomalies propagate faster than theoretical long Rossby waves, and that motion is roughly nondispersive [Chelton and Schlax, 1996]. Several extensions to the linear Rossby theory were constructed to explain the discrepency. See Maharaj et al. [2007] for a comparison of such theories. Zang and Wunsch [1999] show that there is significant energy consistent with the most basic theory for Rossby wave propagation. More recently, attention has focused on mesoscale coherent vortices, which make a significant contribution to the total variability [Fu et al., 2010; Chelton et al., 2011].

The approach in this chapter is highly descriptive. We have no particular theory for ocean variability to test. Instead, the goal is to show that the three-dimensional spectrum contains significant information that is not present in one-dimensional spectra, and to describe the apparent structure in the three-dimensional spectrum of sea surface height variability. We find that the spectrum has a qualitatively universal shape over most of the ocean interior (away from strong currents and the equator). The broad summary of the sea surface height spectrum given here is a first step towards constructing an empirical model of low-frequency ocean variability. The observed spectrum will serve as a "backbone" for the final model.

We begin with a description of the altimetric data and methods used. Section 2.2 describes the three-dimensional spectrum for a typical ocean region in detail. In the Section 4.4, we will consider geographical changes in the shape of the spectrum. Section 2.5 summarizes the results. 


\subsection{Data and methods}

The altimeter data used in this study are "reference" series maps of sea surface height anomaly ( $\mathrm{SSH}$ ) distributed by AVISO. This data product is produced by combining observations from the TOPEX/POSEIDON (T/P), Jason, and ERS satellite altimeters by objective mapping on a $1 / 3^{\circ}$ Mercator grid and subsequent interpolation to a uniform $1 / 4^{\circ} \times 1 / 4^{\circ}$ grid [Le Traon et al., 1998]. Weekly maps are produced, with the anomaly defined relative to the 1993-1999 record mean [AVISO, 2012]. Data used here span the period from October 1992 to December 2010 that was available in March 2011.

The $\mathrm{T} / \mathrm{P}$ and Jason altimeters have a ground track spacing of $316 \mathrm{~km}$ at the equator, with a repeat period of 9.916 days [Fu and Cazenave, 2001; Fu et al., 1994]. For the ERS altimeter, the ground track spacing is $80 \mathrm{~km}$ at the equator and the repeat period is 35 days [Le Traon et al., 1995]. The sampling pattern means that ocean tides are aliased by the altimeter. Tides are removed based on tidal models, though there is some residual tidal signal [Fu et al., 1994; AVISO, 2012, and references therin]. Signals due to ionospheric and atmospheric effects, including water vapor and the inverted barometer effect [Wunsch and Stammer, 1997] are also removed as far as possible.

We use a mapped altimeter data product. The goal of the mapping process is to simultaneously improve the accuracy of the ERS record and improve on the acrosstrack resolution of the $\mathrm{T} / \mathrm{P}$ orbit. One of the benefits of the mapping is to reduce long-wavelength errors due to orbit uncertainty [AVISO, 2012]. The tradeoff is that the merged data product loses the high along-track resolution of the single mission record, and may produce artifacts or obscure features in the original data.

The objective mapping procedure used to create the AVISO gridded data product are described in detail by Le Traon et al. [1998, 2003]. For our purposes, it is most important to note that they apply a covariance function with geographically varying propagation velocities, wavelengths, and periods. Though the exact propagation ve-

locities, periods, and wavelengths used are not shown, Le Traon et al. [2003] say that 
they are "similar to the ones described by Jacobs et al. [2001]." Jacobs et al. [2001] estimated the three dimensional covariance function for mesoscale variability from altimetry, using a high-pass filter to define the mesoscale signal. At each location in their global grid, these authors fit a three-dimensional Gaussian to the estimated covariance function, with zonal, meridional, and temporal $e$-folding scales determined by least squares minimization. Similar $e$-folding scales are used in the AVISO gridding procedure.

The objective mapping procedure smooths variations smaller than the imposed covariance length or time, significantly reducing the spectral power at high wavenumber and frequency. We compare the along-track wavenumber spectrum for $\mathrm{T} / \mathrm{P}$ arc 008 (with an equator crossing longitude of $180^{\circ}$ ) between $30^{\circ} \mathrm{N}$ and $40^{\circ} \mathrm{N}$ with the spectrum of AVISO data interpolated along the same track (Fig. 2-1). The spectrum from the interpolated AVISO data product falls off faster than the $\mathrm{T} / \mathrm{P}$ along-track spectrum, such that the latter exceeds the former by at least a factor of two for wavelengths smaller than $225 \mathrm{~km}$. Similarly, comparison of frequency spectra from $\mathrm{T} / \mathrm{P}$ and the AVISO data product at $35^{\circ} \mathrm{N}, 165^{\circ} \mathrm{E}$ show that the AVISO spectrum is reduced by at least a factor of two for all periods shorter than 55 days (Fig. 2-2).

Despite the loss of energy at small periods and wavelengths in the AVISO data product, it seems to be the best choice for this study due to the ease of computing zonal and meridional wavenumber spectra independently, and because of the increased across-track resolution relative to T/P data alone. ${ }^{1}$ Note that high frequency and wavenumber spectral slopes estimated from the AVISO gridded data product will be unrealistically steep. Fortunately, $X u$ and Fu [2012] have recently estimated wavenumber spectral slopes from Jason-1 along-track altimetry for 70-250 km wavelengths. We do estimate the first moment of the spectrum from the AVISO data product. The objective mapping mostly impacts the low-power part of the spectrum

\footnotetext{
${ }^{1}$ Zonal and meridional spectra can be computed from irregularly spaced data, such as the alongtrack altimetry, as in Glazman and Weichman [2005]. They essentially estimate the autocorrelation function at a range of space-time lags and Fourier transform the result to estimate the frequencywavenumber spectrum. However, this is likely to be very computationally intensive for a global analysis. A more straightforward approach would be to perform a least squares fit of the Fourier series to the data, estimating the Fourier coefficients directly.
} 


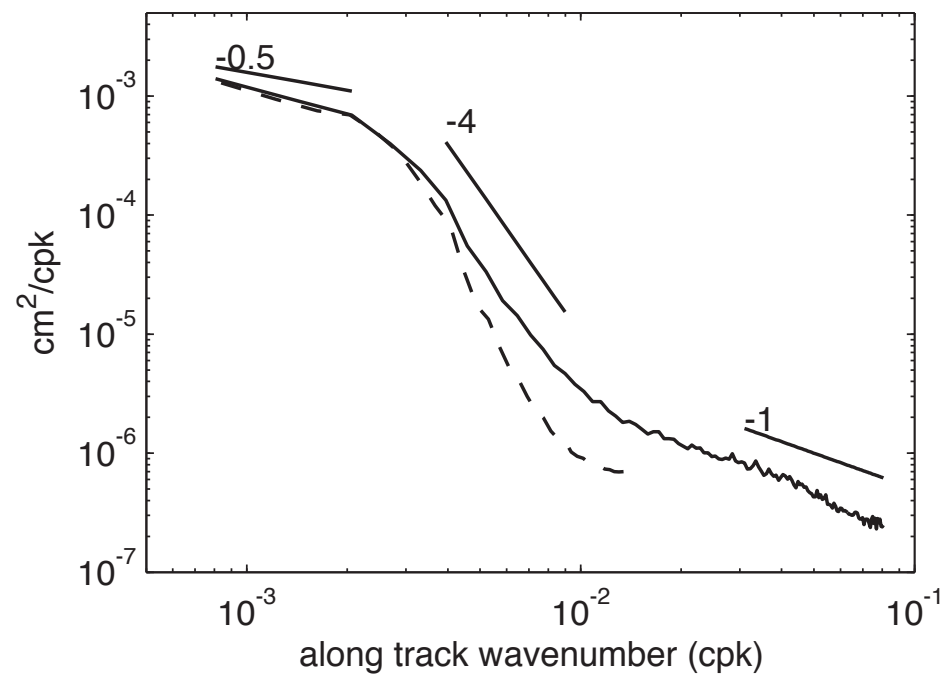

Figure 2-1: The along-track wavenumber spectrum from T/P (solid) and from the AVISO gridded product interpolated along the same track (dashed). Due to the smoothing imposed in the objective mapping procedure, the AVISO product has significantly lower energy at wavelengths shorter than $225 \mathrm{~km}$. Both spectra are along $\mathrm{T} / \mathrm{P}$ arc 008 (equatorial crossing longitude $180^{\circ}$ ) between $30^{\circ} \mathrm{N}$ and $40^{\circ} \mathrm{N}$. Power laws are shown for reference.

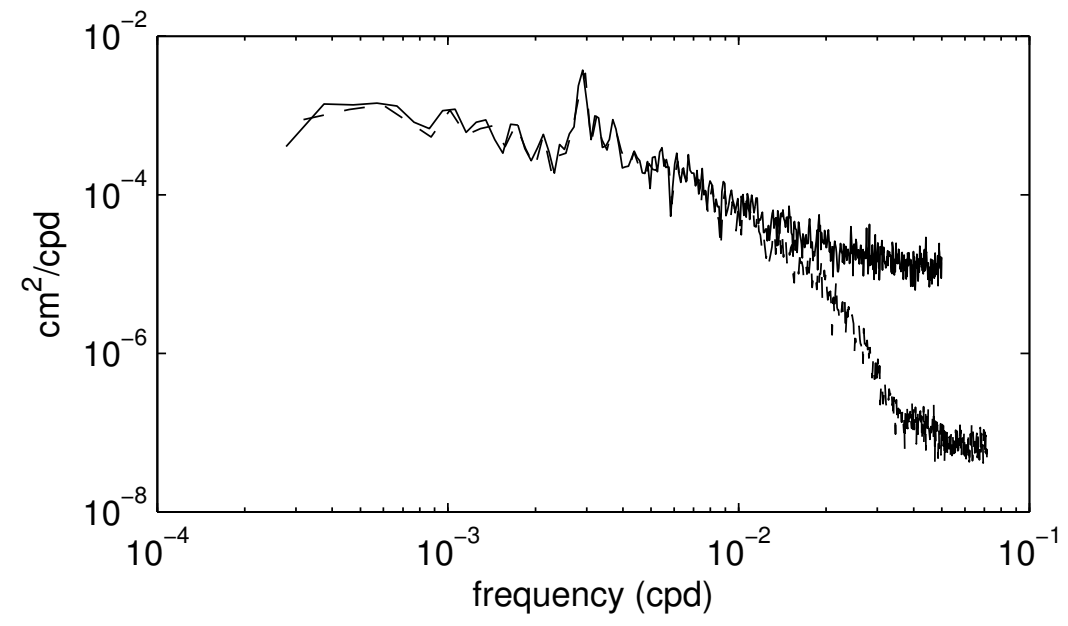

Figure 2-2: The frequency spectrum from $\mathrm{T} / \mathrm{P}$ (solid) and from the AVISO gridded product (dashed) at $35^{\circ} \mathrm{N}, 165^{\circ} \mathrm{E}$. Due to the smoothing imposed in the objective mapping procedure, the AVISO product has significantly lower energy at periods shorter than 55 days. 
and has little impact on the first moment. Comparison of the first moment of the spectrum from the along-track and AVISO gridded altimetry indicate a change of less than $10 \%$.

Moving beyond issues of data handling, there is the question of what the satellite altimeter sees. The conventional view is that the altimeter primarily reflects geostrophically balanced motions, and low baroclinic mode Rossby waves [Chelton and Schlax, 1996] in particular. The barotropic mode Rossby wave propagates across an ocean basin in about a week, too fast for an orbiting satellite. First baroclinic mode waves are much slower, requiring months to decades to cross an ocean basin (Fig. 2-3). Poleward of about $37^{\circ}$, the altimetric record is not currently long enough to have seen a baroclinic wave cross the Pacific ocean.

Internal waves are likely to contribute to the SSH signal. Since internal waves are confined to frequencies between the Coriolis parameter $f$ and buoyancy frequency $N$, they would be aliased in frequency space. However, there is no wavenumber cutoff for internal waves, and sufficiently long internal waves would not be averaged out by the altimeter sampling pattern. While internal tides are largely removed, the background internal wave continuum [Garrett and Munk, 1975] is not. To the best of our knowledge, the degree to which non-tidal internal waves are present in the SSH signal has not been explored.

As noted by Stammer [1997], ocean surface eddy kinetic energy is very inhomogeneous, with high energy in the boundary currents, in the tropics, and Southern Ocean, but lower energy within the subpolar and subropical gyres. As a result, SSH data are analyzed for separate boxes, spanning $30^{\circ}$ of longitude by $10^{\circ}$ of latitude, chosen to balance homogeneity and resolution. ${ }^{2}$ All weekly SSH maps for a given region are extracted, small gaps near islands are filled by linear interpolation, the spatial mean and linear trend in latitude and longitude are removed at each time step, and the three-dimensional FFT is computed for the region. Removing the spatial mean at each time step effectively removes the seasonal cycle since it has large

\footnotetext{
${ }^{2}$ The conventional theory assumes spatial and temporal stationarity. This is clearly not the case here, at least spatially. The power spectrum is still useful, but a complete description also involves the bispectrum, trispectrum, and higher-order spectra.
} 


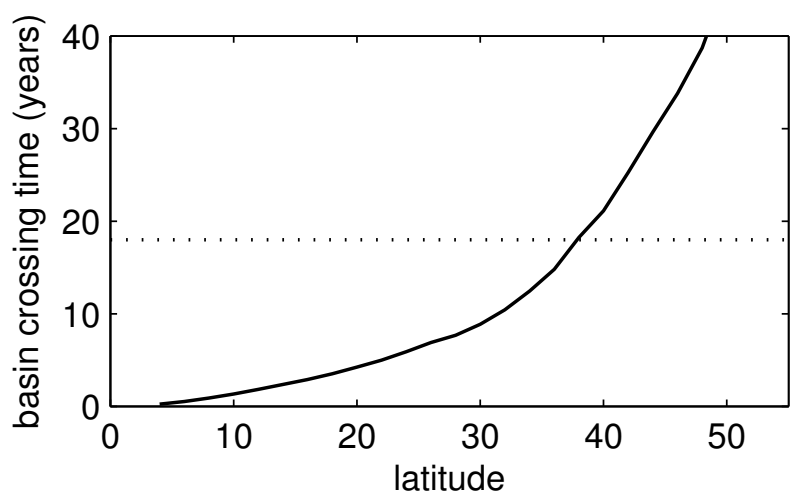

Figure 2-3: The time required for a long baroclinic Rossby wave to cross a basin $100^{\circ}$ wide (solid), roughly the width of the Pacific. The 18 year altimetric record (dashed) used in this study is sufficient to record Rossby waves crossing the Pacific equatorward of $35^{\circ}$. Basin transit times are calculated based on the basic theory for free waves in a flat-bottom, resting ocean [Chelton et al., 1998].

wavelength, but no other filtering is performed. Using other windowing functions did not significantly alter the results. Power spectra are computed from the FFT, averaged over three neighboring frequency/wavenumber bands (for an approximate 54 degrees of freedom), and normalized such that the total power in the spectrum $\iiint \Phi_{\eta}(k, l, \omega) \mathrm{d} \omega \mathrm{d} l \mathrm{~d} k$ is equal to the record variance. Units for zonal wavenumber $k$ and meridional wavenumber $l$ are cycles per kilometer (cpk), and frequency $\omega$ is in cycles per day (cpd).

The three-dimensional spectrum $\Phi_{\eta}(k, l, \omega)$ is a powerful tool that distinguishes between standing and propagating variability, as well as various wavelengths at a given frequency. Unfortunately, the full three-dimensional spectrum is difficult to display and one- or two-dimensional marginal spectra will also be used, e.g.

$$
\begin{aligned}
& \Phi_{\eta}(k, l)=\int \Phi_{\eta}(k, l, \omega) \mathrm{d} \omega \\
& \Phi_{\eta}(k)=\iint \Phi_{\eta}(k, l, \omega) \mathrm{d} \omega \mathrm{d} l
\end{aligned}
$$

If the SSH variability is a white noise process, the $100(1-\alpha) \%$ confidence interval 
for $\Phi_{\eta}(k, l, \omega)$ is [Chatfield, 2004, §7.5]

$$
\frac{\nu \Phi_{\eta}}{\chi_{\nu, \alpha / 2}^{2}}<\tilde{\Phi}_{\eta}<\frac{\nu \Phi_{\eta}}{\chi_{\nu,(1-\alpha / 2)}^{2}}
$$

with $\nu \sim 54$ degrees of freedom, where $\tilde{\Phi}_{\eta}$ is the (unknown) true value. This serves as a rough estimate of the confidence interval for the three-dimensional spectrum.

The marginal spectrum $\Phi_{\eta}(k, l)$ is computed by summing $\Phi_{\eta}(k, l, \omega)$ over all frequencies, and it is nontrivial to estimate the number of degrees of freedom directly. The marginal spectra are asymptotically distributed as $\chi_{\nu^{\prime}}^{2}$ variables. Naively, $\nu^{\prime}$ might be assumed to be the number of elements summed over, multiplied by $\nu$. However, the power spectrum $\Phi_{\eta}(k, l, \omega)$ is not white, so only a small number of elements effectively determine the marginal spectrum $\Phi_{\eta}(k, l)$, reducing the effective number of degrees of freedom $\nu^{\prime}$. Instead, confidence intervals are shown for the two-dimensional $k$ - $l$ spectrum averaged over all snapshots. The number of degrees of freedom for the two-dimensional spectrum is estimated assuming that snapshots separated by more than 100 days are independent, based on the the first zero crossing of the autocorrelation function [Stammer, 1997]. Confidence intervals for other marginal spectra are calculated similarly, using a space correlation wavelength of $250 \mathrm{~km}$.

\subsection{Observed spectrum from altimetry}

Eddy kinetic energy varies by about four orders of magnitude over the ocean (Fig. 24). Despite this fact, there appears to be a consistent shape to the two-dimensional $\omega-k$ spectrum of SSH. We begin with a detailed look at the spectrum of SSH for a typical region in the North Pacific, and then describe the features of the spectrum that are common to much of the interior ocean.

\subsubsection{Typical SSH spectrum}

The North Pacific around $30^{\circ} \mathrm{N}, 190^{\circ} \mathrm{E}$ is chosen as a typical low energy region of the ocean, and SSH data for a box spanning $30^{\circ}$ of longitude and $10^{\circ}$ of latitude 


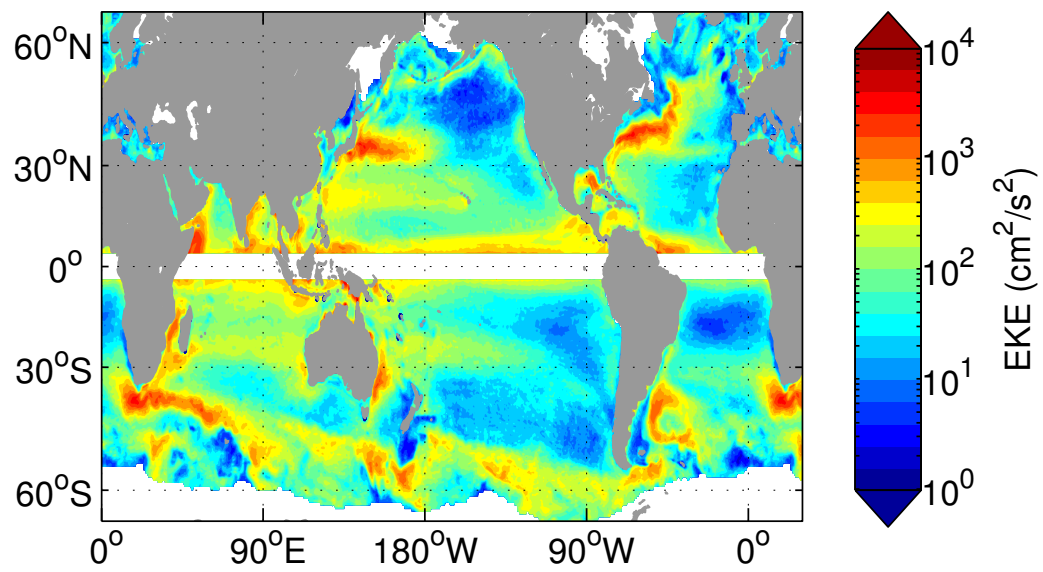

Figure 2-4: Eddy kinetic energy, calculated from AVISO altimetry, varies by over four orders of magnitude over the ocean.

is considered (Fig. 2-5). Westward propagating features, whether Rossby waves or coherent vortices, have received much attention, and stand out clearly in longitudetime plots [Chelton and Schlax, 1996; Chelton et al., 2007; Fu, 2009]. In contrast to the longitude-time plot, the latitude-time plot shows no hint of systematic meridional propagation.

Following Hill et al. [2000] and Challenor et al. [2001], we estimate the dominant zonal phase speed using the full two-dimensional Radon transform of the longitudetime plot at fixed latitude. Let $L$ be an arbitrary line oriented at an angle $\theta$ relative to the longitude axis. The Radon transform is defined as the projection $p(s, \theta)$ of the $\mathrm{SSH}$ onto $L$, where $s$ is the coordinate along $L$ (Fig. 2-6). When the line $L$ is perpendicular to the ridges and crests of the longitude-time plot, the variance of the projection will be maximized. Thus, we find the angle $\theta_{c}$ that maximizes the variance of $p(s, \theta)$. The dominant phase speed is $c_{p x}=\Delta x / \Delta t \times \cot \left(\theta_{c}\right)$, where $\Delta x$ and $\Delta t$ are the spatial and temporal sampling rates for the SSH data. Here, the dominant speed is $3.9 \mathrm{~km} /$ day westward.

The three-dimensional wavenumber-frequency spectrum for this region is difficult to visualize, but three features are worth noting (Fig. 2-7). First, the most conspicuous feature of the three-dimensional spectrum is the dominance of westward propagation (negative zonal wavenumber), as illustrated by the red surface of constant 


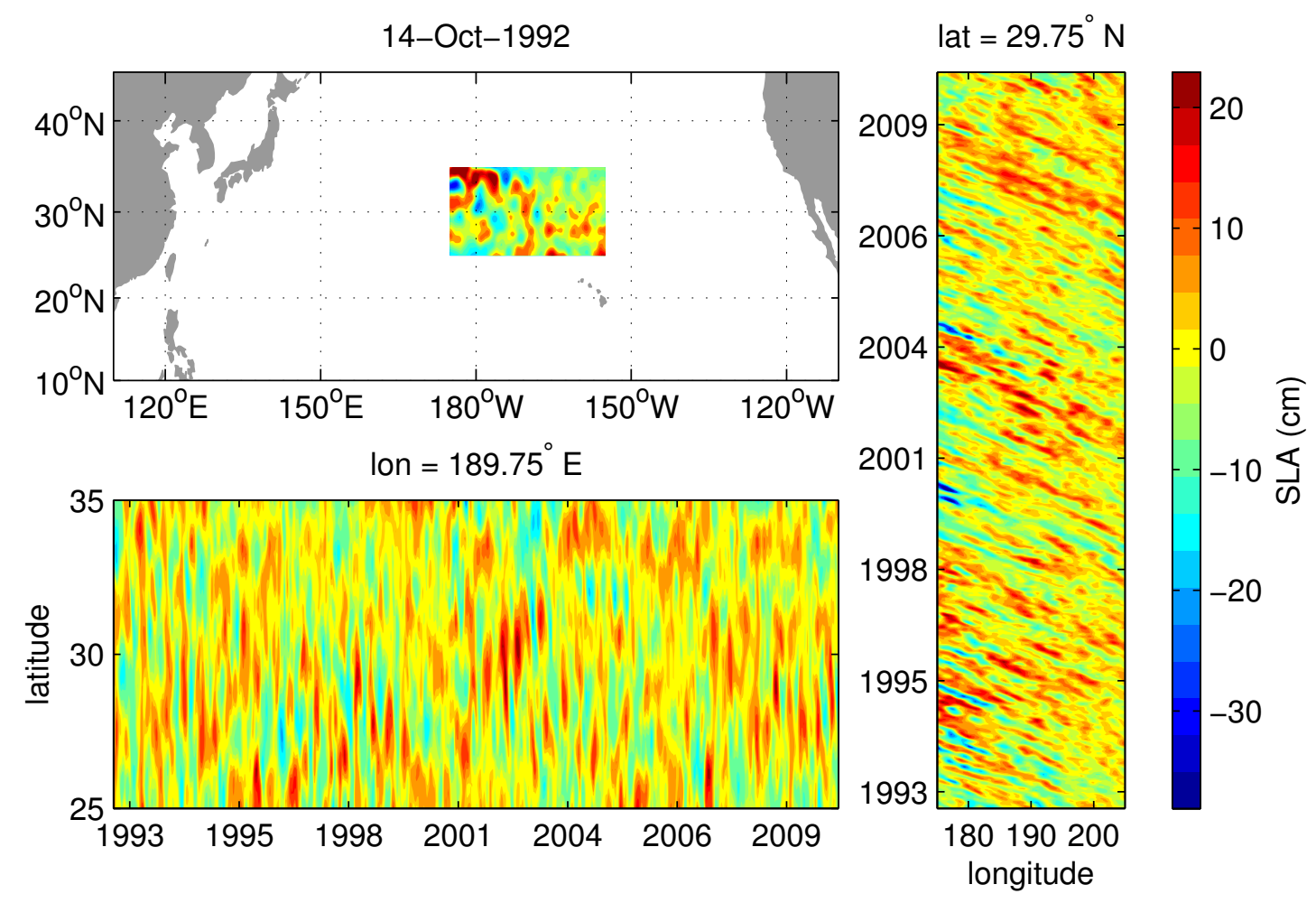

Figure 2-5: Snapshot of SSH map used to compute spectra (top left). The longitudetime plot (right) shows the dominance of westward propagation of SSH anomalies, while the latitude-time plot (bottom left) does not show a clear pattern of meridional propagation.

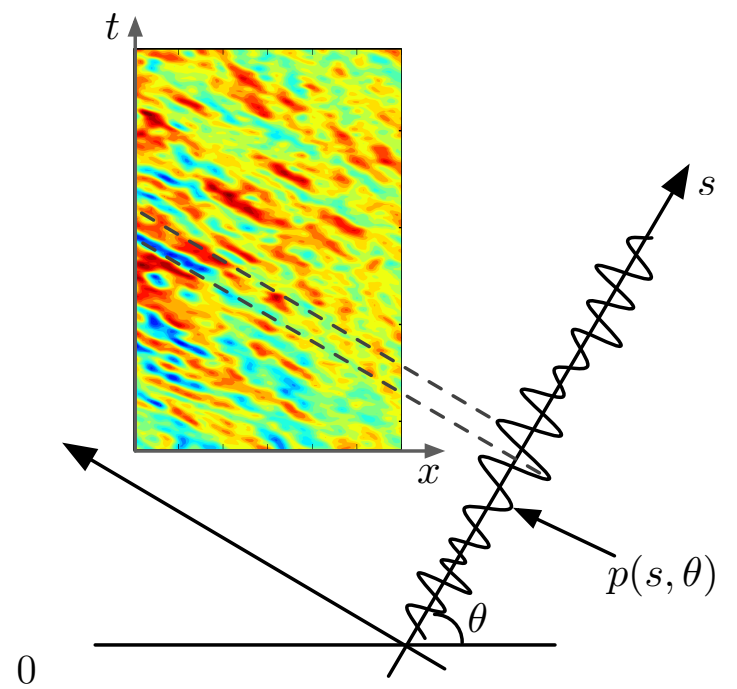

Figure 2-6: The Radon transform of the longitude-time plot of SSH (colored) is the projection $p(s, \theta)$ of the signal onto a line $L$ at angle $\theta$ relative to the longitude axis. The variance of $p(s, \theta)$ maximized when the line $L$ is perpendicular to the ridges and crests of the longitude-time plot, indicating the dominant phase speed. 


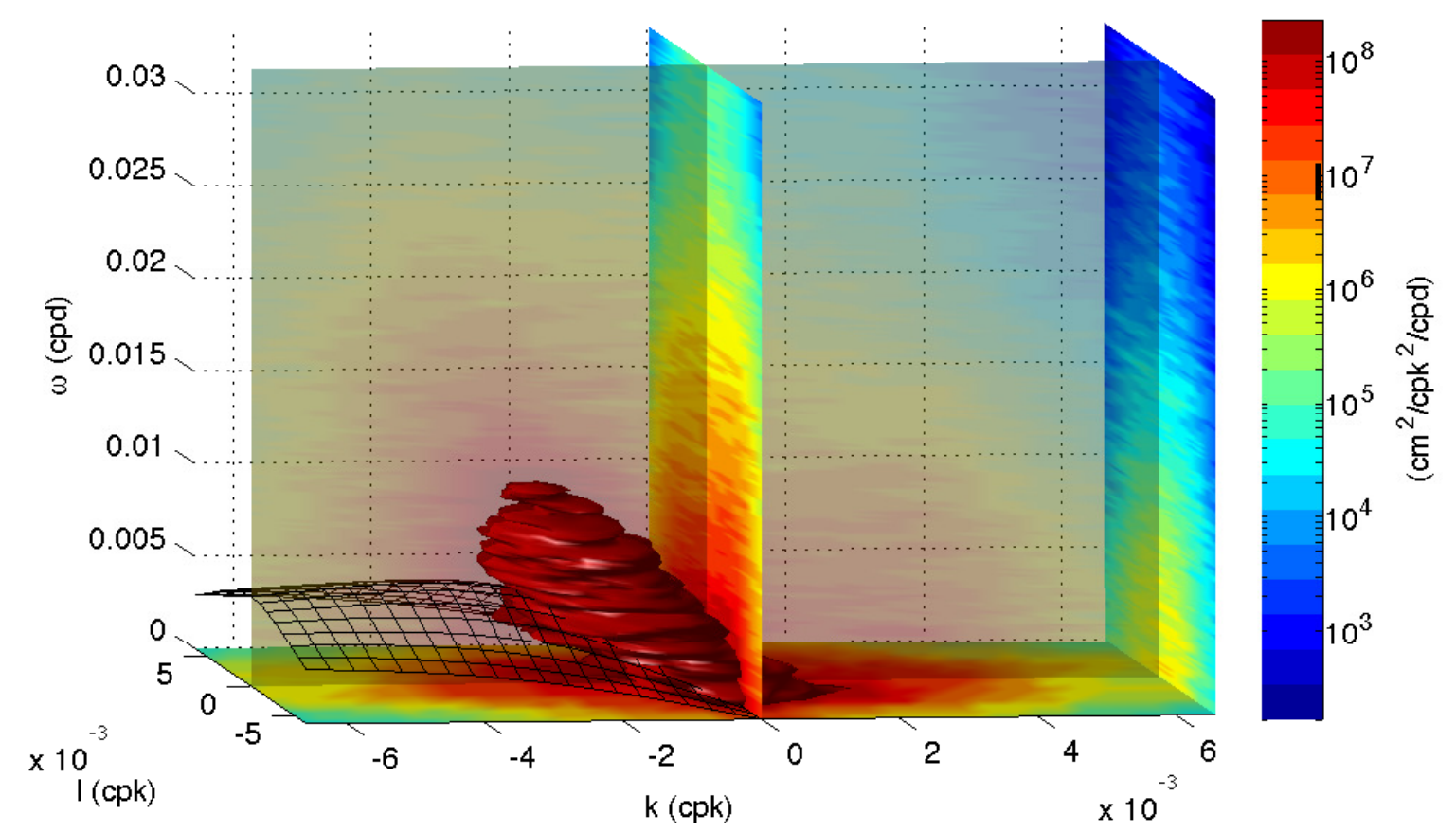

Figure 2-7: The three-dimensional wavenumber-frequency spectrum for the region near $30^{\circ} \mathrm{N}, 190^{\circ} \mathrm{E}$. Slices through the planes $w=0, l=0, k=0$, and $k=6.6 \times$ $10^{-3} \mathrm{cpk}$ are shown. The red surface of constant power illustrates the dominance of westward propagation. For reference, the mesh surface shows the dispersion relation for classical theory of baroclinic Rossby waves. The black bar on the colorscale indicates a $95 \%$ confidence interval.

power. Second, the dominant phase speed does not follow the dispersion relation for basic linear baroclinic Rossby waves with no mean flow (the classical theory). Instead, the dominant speed appears independent of wavenumber; the propagation of $\mathrm{SSH}$ features is nondispersive. Third, the dominant phase speed appears independent of meridional wavenumber $l$. This can be seen by rotating the three-dimensional spectrum (easy on a computer but difficult on paper) or by taking slices through the space at various meridional wavenumbers. ${ }^{3}$ In three-dimensions, the surface of constant power appears as a tongue, thin in the $k$-dimension but wide in $l$, stretching through the $k<0$ half space.

Despite the initial focus on anomalously fast Rossby wave propagation [Chelton

\footnotetext{
${ }^{3} \mathrm{~A}$ short animation of the three-dimensional spectrum is available at http://worthamc.scripts.mit.edu/home/academic/ and http://vimeo.com/35066699.
} 


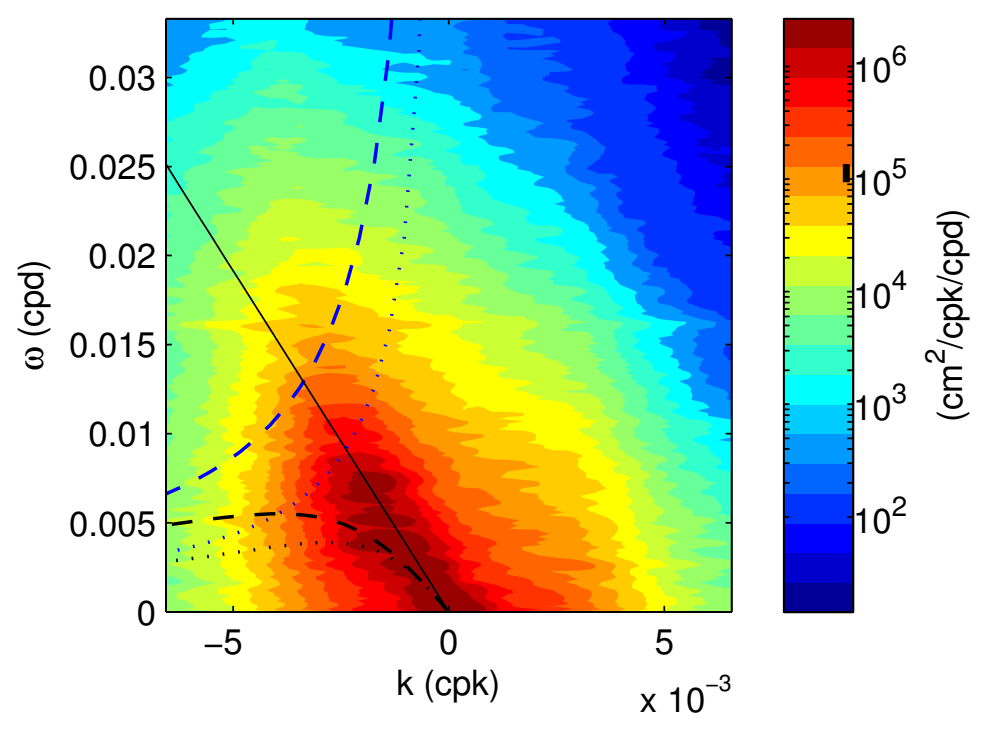

Figure 2-8: Two-dimensional zonal wavenumber-frequency spectrum for the region near $30^{\circ} \mathrm{N}, 190^{\circ} \mathrm{E}$. Dispersion curves for basic theory barotropic (blue) and baroclinic (black) Rossby waves with no mean flow are shown with $l=0$ (dashed) and $l=k$ (dotted). The solid black line shows the dominant phase speed as found from a Radon transform of the longitude-time plot.

and Schlax, 1996], more recent studies have focused on mesoscale ${ }^{4}$ coherent vortices [Fu et al., 2010; Chelton et al., 2011]. The two-dimensional zonal wavenumberfrequency spectrum (Fig. 2-8) shows that the dominant phase speed follows a straight "ridge" of westward variance, with propagation faster than the classical theory long wave speed at all wavelengths. The search for an explanation for the propagation speed of SSH features with wavelengths $\mathcal{O}(1000 \mathrm{~km})$ remains open, and will be addressed in Ch. 4.

The meridional wavenumber-frequency spectrum is centered on $l=0$ with a large contribution from nonzero wavenumber (Fig. 2-9). There is nearly equal energy propagating northward $(l>0)$ and southward $(l<0)$, but no hint energy is confined to a classical Rossby wave dispersion relation.

The two-dimensional wavenumber spectrum (Fig. 2-10) shows that westward propagation dominates. The peak of the wavenumber spectrum is at $k=-1.7 \times 10^{-3} \mathrm{cpk}$, $l=0$, corresponding to westward propagation with a wavelength of $575 \mathrm{~km}$.

\footnotetext{
${ }^{4}$ We define mesoscale variability as having wavelengths of tens to hundreds of $\mathrm{km}$ and periods of tens to hundreds of days.
} 


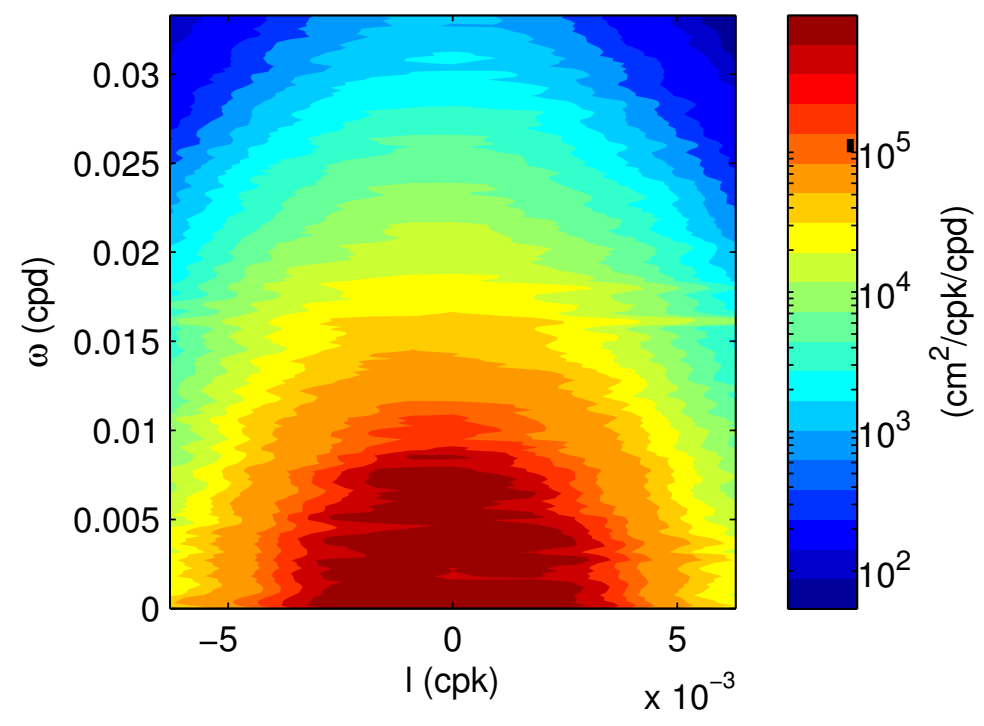

Figure 2-9: Two-dimensional meridional wavenumber-frequency spectrum for the region near $30^{\circ} \mathrm{N}, 190^{\circ} \mathrm{E}$.

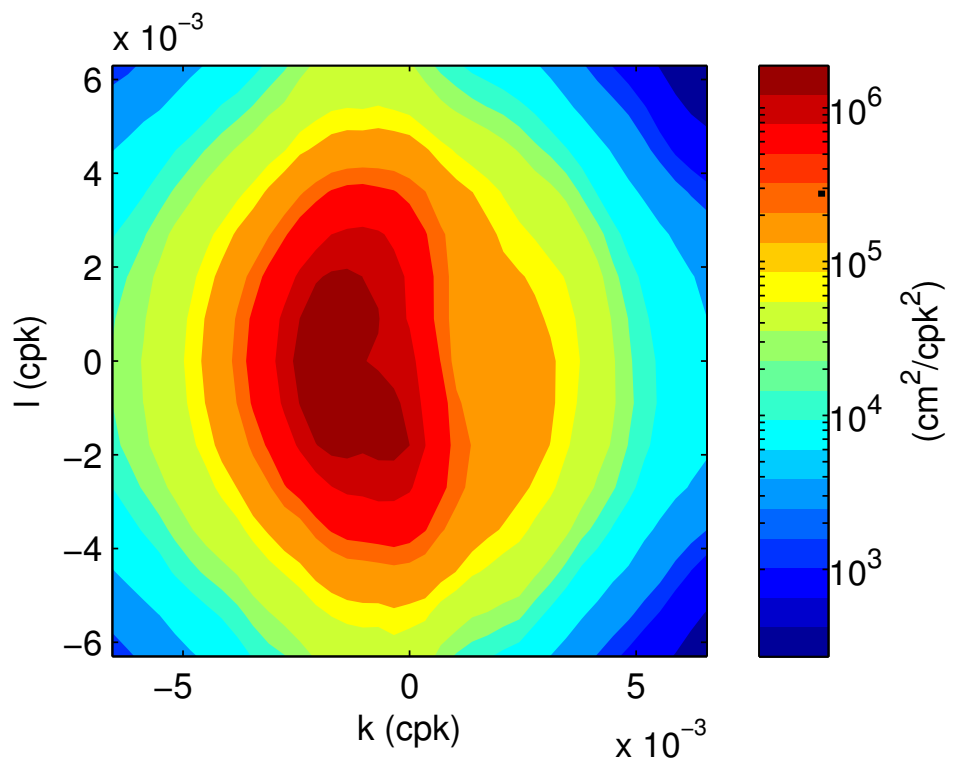

Figure 2-10: Two-dimensional zonal and meridional wavenumber spectrum for the region near $30^{\circ} \mathrm{N}, 190^{\circ} \mathrm{E}$. Westward propagation is clearly dominant, and there is a slight excess of southward propagation. 
Taken together, the two- and three-dimensional spectra contain much information about the scales and propagation of SSH features in this region. While all frequencies, wavenumbers, and phase speeds are present, westward propagation with a constant phase speed dominates at all frequencies. Meridional propagation is apparent, with northward and southward motion roughly balanced.

One-dimensional spectra for frequency and wavenumber are shown in Fig. 2-11. Positive and negative zonal wavenumber spectra are shown separately, and negative wavenumbers dominate at all scales. At large scales, the positive wavenumber spectrum has a spectral slope near $k^{-1 / 2}$, while the negative wavenumber spectrum is slightly "blue", with a peak at $1.7 \times 10^{-3} \mathrm{cpk}$. At small scales, both positive and negative zonal wavenumber spectra approach a $k^{-5}$ power law, but this is an artifact of the AVISO objective mapping procedure. Meridional wavenumber spectra are separated into northward and southward components, showing only a small difference in amplitude. Both components are slightly "red" at low wavenumber, gradually transitioning to steeper slopes at high wavenumber.

The frequency spectrum is slightly "red" at low frequency, transitioning to a steeper power law near $7 \times 10^{-3} \mathrm{cpd}$. The frequency spectrum is partitioned into eastward, westward, and standing components by integrating the three-dimensional spectrum over positive $k$, negative $k$, and $k=0$ independently. Energy is partitioned equally between eastward and westward motion for periods longer than 10 years, but westward motion dominates at all shorter periods.

The focus on a particular wavenumber or phase speed, here and in much of the literature, obscures an important point: ocean variability is broad band in frequency and wavenumber. Despite the focus on a particular region of wavenumber-frequency space in Fig. 2-7, there is ocean signal at all frequencies and wavenumbers. Two examples illustrate the importance of considering the full spectrum. 1) While high frequencies and wavenumbers may only contain a small fraction of the total variance, they are important for energy flux calculations and impact the interpretation of ocean energetics [e.g. Scott and Wang, 2005]. 2) The presence of significant energy at low frequency and high wavenumber in Fig. 2-8 implies the existence of persistent small- 

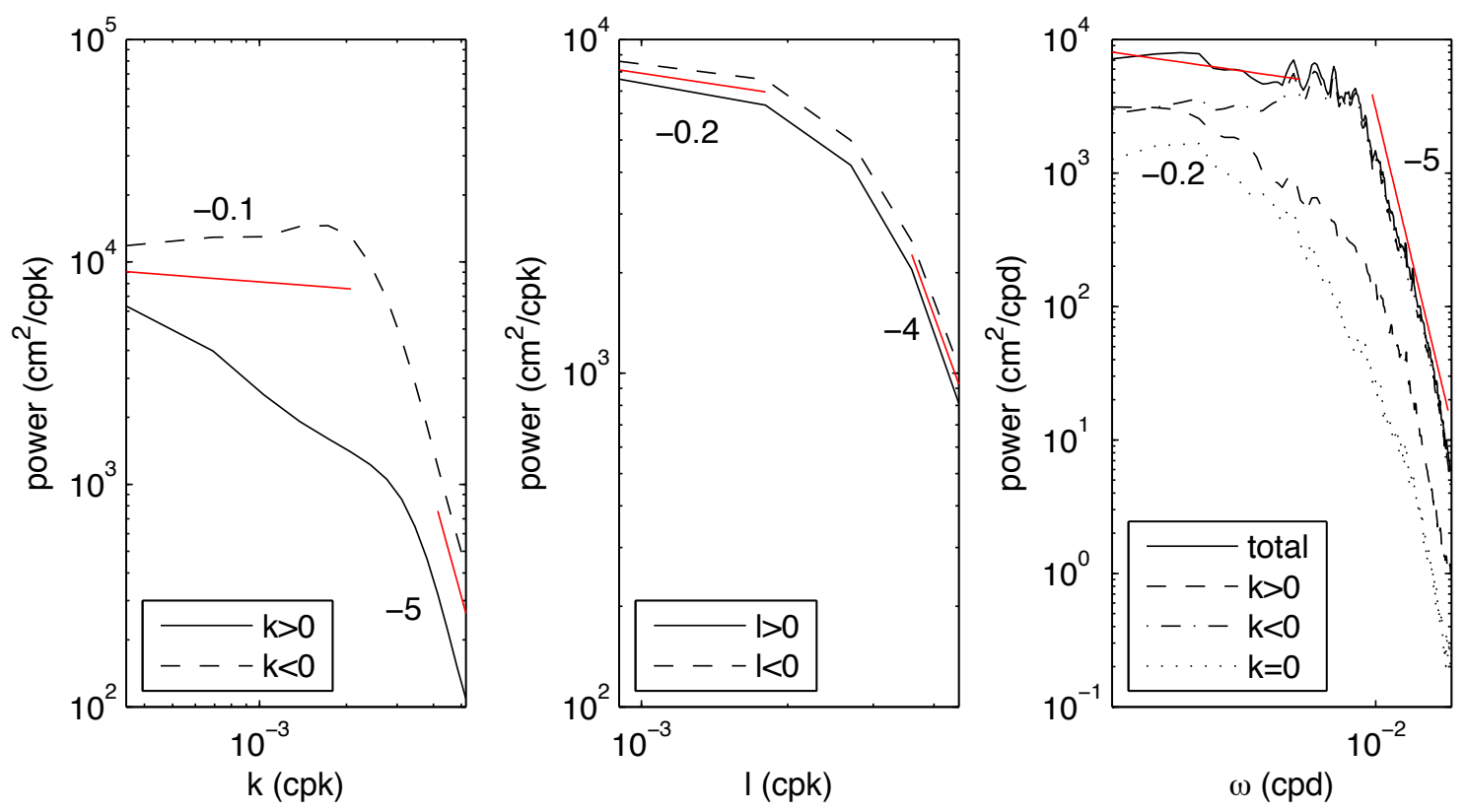

Figure 2-11: One-dimensional zonal wavenumber (left) meridional wavenumber (center) and frequency (right) spectra for the region near $30^{\circ} \mathrm{N}, 190^{\circ} \mathrm{E}$. For zonal wavenumber, westward propagation (dashed) is stronger than eastward (solid) over most of the ocean. For meridional wavenumber, southward propagation (dashed) is stronger than northward (solid) in this region, but the imbalance is relatively small and regionally variable. The frequency spectrum is further divided into eastward (dash), westward (dash-dotted), and standing (dotted) components based on zonal wavenumber. Red lines show power laws with the indicated slope. 


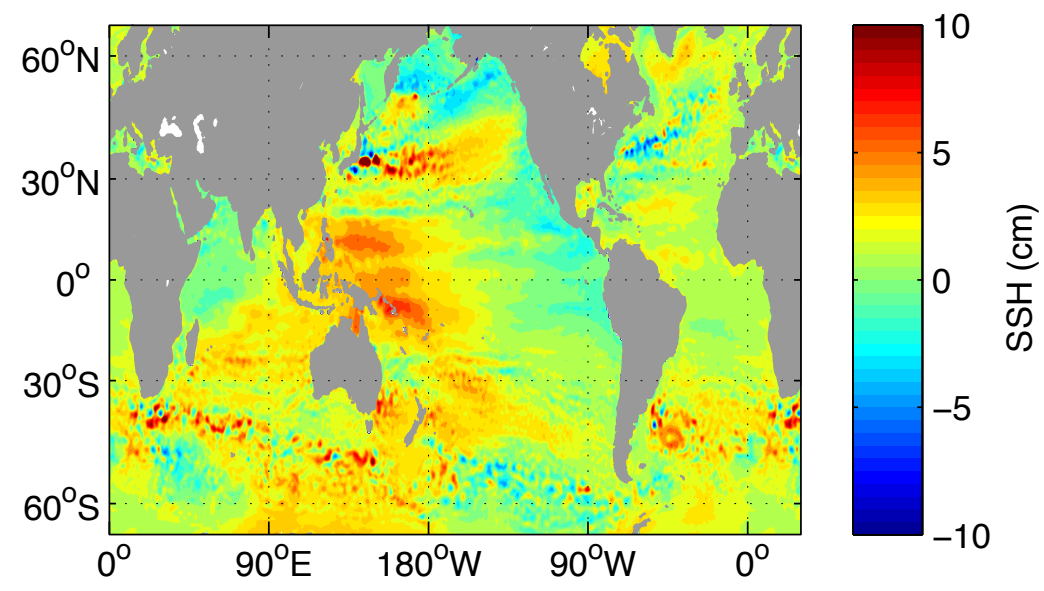

Figure 2-12: The 18-year mean SSH (1992-2010) still shows significant smallwavelength features corresponding to the high wavenumber, low frequency part of the spectrum.

wavelength features in SSH; apparently the time mean is not smooth. As a concrete example, Fig. 2-12 shows the 18-year mean SSH anomaly. (Details of Fig. 2-12 change for different averaging periods, suggesting that these are not permanent eddies, but very persistent features.)

\subsubsection{Kinetic energy spectrum}

So far, we have focused on the SSH spectrum. However, velocity and kinetic energy are typically the more dynamically interesting quantities and their spectra are predicted by turbulence theories, for example. Assuming the flow is geostrophic, near-surface velocities can be calculated from the SSH field $\eta(x, y)$ by

$$
\begin{aligned}
f_{0} v(x, y) & =g \frac{\partial \eta(x, y)}{\partial x} \\
-f_{0} u(x, y) & =g \frac{\partial \eta(x, y)}{\partial y}
\end{aligned}
$$



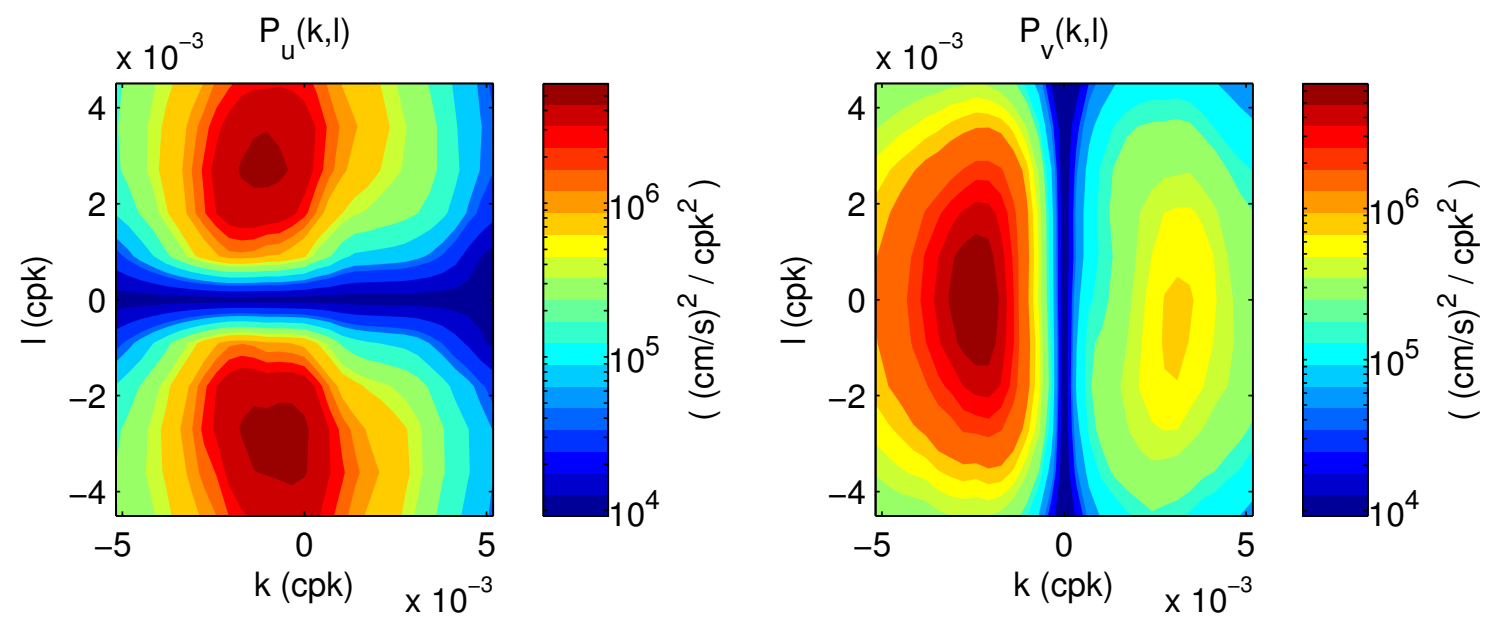

Figure 2-13: Two-dimensional wavenumber spectra of zonal (left) and meridional (right) velocity for the region near $30^{\circ} \mathrm{N}, 190^{\circ} \mathrm{E}$. Westward propagation dominates for both velocity components, but the spectra are approximately symmetric in the meridional direction.

Taking a two-dimensional Fourier transform with respect to $x$ and $y$, one can easily show that the two-dimensional velocity spectra are related to the SSH spectrum by

$$
\begin{aligned}
& \Phi_{v}(k, l)=\frac{4 \pi^{2} g^{2}}{f_{0}^{2}} k^{2} \Phi_{\eta}(k, l) \\
& \Phi_{u}(k, l)=\frac{4 \pi^{2} g^{2}}{f_{0}^{2}} l^{2} \Phi_{\eta}(k, l)
\end{aligned}
$$

The four one-dimensional velocity spectra $\left(\Phi_{u}(k), \Phi_{u}(l), \Phi_{v}(k), \Phi_{v}(l)\right)$ are obtained by integrating the two-dimensional spectra over one component of wavenumber. The two-dimensional wavenumber spectra of velocity are shown in Fig. 2-13. As expected, westward propagation dominates for both velocity components, but the spectra are approximately symmetric in the meridional direction.

We note two interesting kinematic features of the observed spectrum, and the relation between the SSH and velocity spectra in particular. 1) The SSH spectrum $\Phi_{\eta}(k, l)$ must remain finite as $(k, l) \rightarrow 0$. A simple model for the spectrum is $\Phi_{\eta}(k, l) \sim$ $\left(k^{2}+l^{2}+\epsilon\right)^{-\alpha}$ for $\epsilon>0$. In this case, the zonal wavenumber spectra for zonal and 
meridional velocity are

$$
\begin{aligned}
& \Phi_{u}(k) \sim\left(k^{2}+\epsilon\right)^{(3 / 2-\alpha)} \\
& \Phi_{v}(k) \sim k^{2}\left(k^{2}+\epsilon\right)^{(1 / 2-\alpha)}
\end{aligned}
$$

The rolloff of the SSH spectrum as $k \rightarrow 0$, which is required if the spectrum is to remain finite, forces the zonal and meridional velocity spectra to diverge at low wavenumber. This conclusion applies to spectra computed at any spatial orientation; across- and along-track velocity spectra will always diverge at sufficiently large wavelengths. This divergence can be seen in along- and across-track velocity spectra from shipboard ADCP measurements (J. Callies 2012, personal communication). However, integrating over all track orientations to form the spectrum of wavenumber magnitude $K=\sqrt{k^{2}+l^{2}}$ will result in equal spectra: $\Phi_{u}(K)=\Phi_{v}(K)$. 2) Westward propagation dominates in most of the ocean, making the SSH spectrum anisotropic. A simple form for the spectrum accounting for this anisotropy is $\Phi_{\eta}(k, l) \sim\left((k+\delta)^{2}+l^{2}\right)^{-\alpha}$. The shift $\delta$ breaks the isotropy of the velocity spectrum at large wavelengths.

Scharffenberg and Stammer [2011] estimated along-track spectra of $u$ and $v$ from the $\mathrm{T} / \mathrm{P}-J a s o n$ tandem mission, and found that the spectra of the two velocity components diverge at wavelengths larger than $200 \mathrm{~km}$. Both points (1) and (2) above contribute to the observed divergence of the velocity spectra. However, the Scharffenberg and Stammer [2011] analysis was complicated by the orientation of the satellite track. Since the satellite track is neither zonal nor meridional, their $u$ and $v$ spectra are a linear combination of along- and across-track spectra.

As a slight digression, we note that the signature of zonal striations [Maximenko et al., 2005; Nakano and Hasumi, 2005; Richards et al., 2006] can be seen in the velocity spectra. The $k$ - $l$ spectrum of zonal velocity for the time mean $(\omega=0)$ has a peak at $k=0, l= \pm 3 \times 10^{-3}$, corresponding to zonally extended jets or striations with a meridional spacing of about $300 \mathrm{~km}$ (Fig. 2-14). In contrast, the $k$ - $l$ spectrum of meridional velocity for the time mean has peaks at $k=l= \pm 2 \times 10^{-3} \mathrm{cpk}$, with no indication of a "meridional striation". 

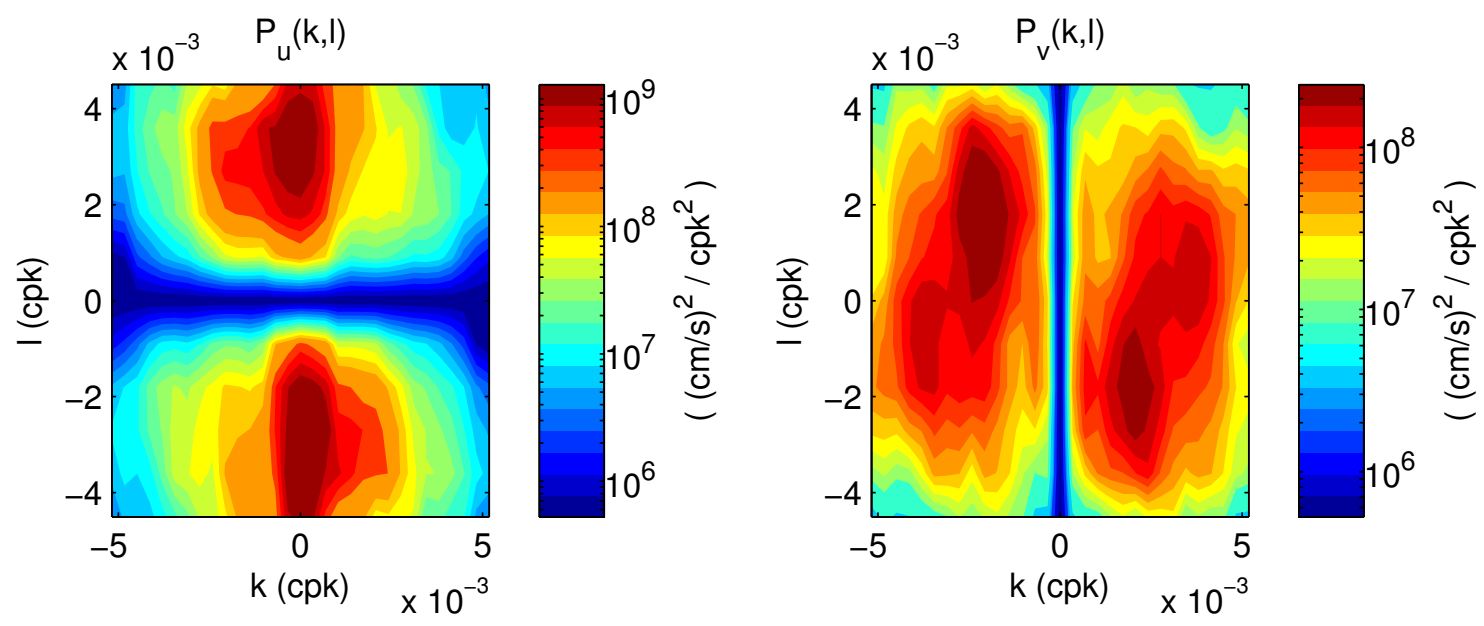

Figure 2-14: Two-dimensional wavenumber spectra of time-mean zonal (left) and meridional (right) velocity for the region near $30^{\circ} \mathrm{N}, 190^{\circ} \mathrm{E}$ shows the signature of zonal striations. Westward propagation dominates for both velocity components, but the spectra are approximately symmetric in the meridional direction. While the spectra in Fig. 2-13 were integrated over all frequencies, here we show the spectra for $\omega^{-1}=18$ years.

The anisotropy of the velocity spectrum carries over to the eddy kinetic energy spectrum $\Phi_{\mathrm{EKE}}(k, l)=1 / 2\left[\Phi_{u}(k, l)+\Phi_{v}(k, l)\right]$ (Fig. 2-15). The spectrum is dominated by a ridge of high power, marked in the figure. The average magnitude of the wavenumbers in the ridge is $K=2.7 \times 10^{-3} \mathrm{cpk}$, corresponding to a wavelength of $370 \mathrm{~km}$. The wave propagation angle $\phi=\tan ^{-1}(l / k)$ ranges from 0 (due westward) to $60^{\circ}$ north and south.

\subsubsection{Universality}

We have attempted to describe a typical region of the ocean, near $30^{\circ} \mathrm{N}, 190^{\circ} \mathrm{E}$, in terms of the spectrum of SSH variability. Here, we describe the aspects of the spectrum that are common to other regions, and aspects that vary geographically.

The most obvious variation is in eddy kinetic energy (Fig. 2-4). Within relatively quiescent regions the main change in the shape of the spectrum is the slope of the ridge of westward variance. Several $k-\omega$ spectra from the North Pacific (along $220^{\circ} \mathrm{E}$ ) and South Atlantic (along $345^{\circ} \mathrm{E}$ ) show that the slope of the ridge increases toward the equator, consistent with the increasing Rossby wave phase speed (Fig. 2-16). 


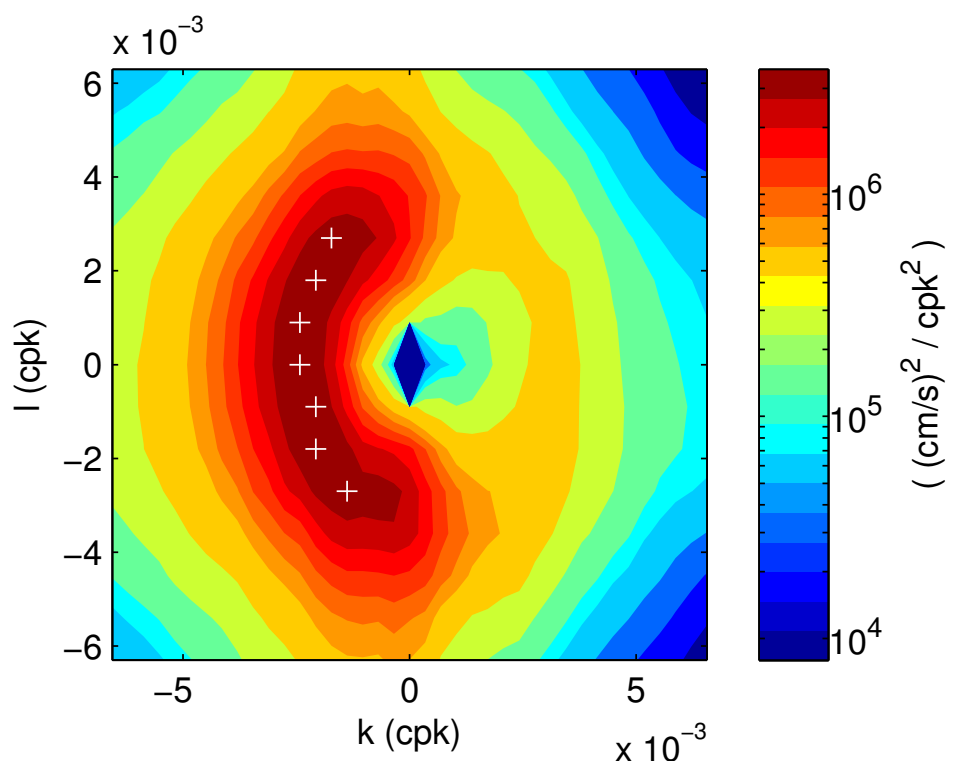

Figure 2-15: Two-dimensional zonal and meridional wavenumber spectrum of EKE for the region near $30^{\circ} \mathrm{N}, 190^{\circ} \mathrm{E}$. White ' + ' mark the peak, in $k$, of the spectrum at selected meridional wavenumbers, tracing a ridge of high power.

The Atlantic spectrum at $40^{\circ} \mathrm{S}$ is a region of significant intermittent eastward flow, associated with meandering of the ACC. The intermittent eastward flow creates excess eastward variance relative to the Pacific spectrum at $40^{\circ} \mathrm{N}$. Near the equator $\left(5^{\circ} \mathrm{N}\right.$ and $\mathrm{S})$, the ridge of westward variance becomes nearly vertical, with variance restricted to large wavelengths but extending to higher frequencies than at other latitudes. The mid-latitude Indian Ocean $\left(20^{\circ} \mathrm{S}, 75^{\circ} \mathrm{E}\right)$ is comparable to other regions at the same latitude.

The Kuroshio and Gulf Stream spectra $\left(35^{\circ} \mathrm{N}, 160^{\circ} \mathrm{E}\right.$ and $35^{\circ} \mathrm{S}, 300^{\circ} \mathrm{E}$, respectively) represent two of the most energetic regions of the ocean. Still, the spectra are dominated by a ridge of westward variance with phase speed appropriate to the latitude. Here, the increased eastward variance is due to the narrow eastward boundary current flow.

Despite the geographic variation in the spectrum, many features of the SSH spectrum described above for the region $30^{\circ} \mathrm{N}, 190^{\circ} \mathrm{E}$ are typical of other regions of the ocean. We propose that the interior ocean, from a few degrees off the equator to $45^{\circ}$ north and south, and outside of western boundary currents, can be described 

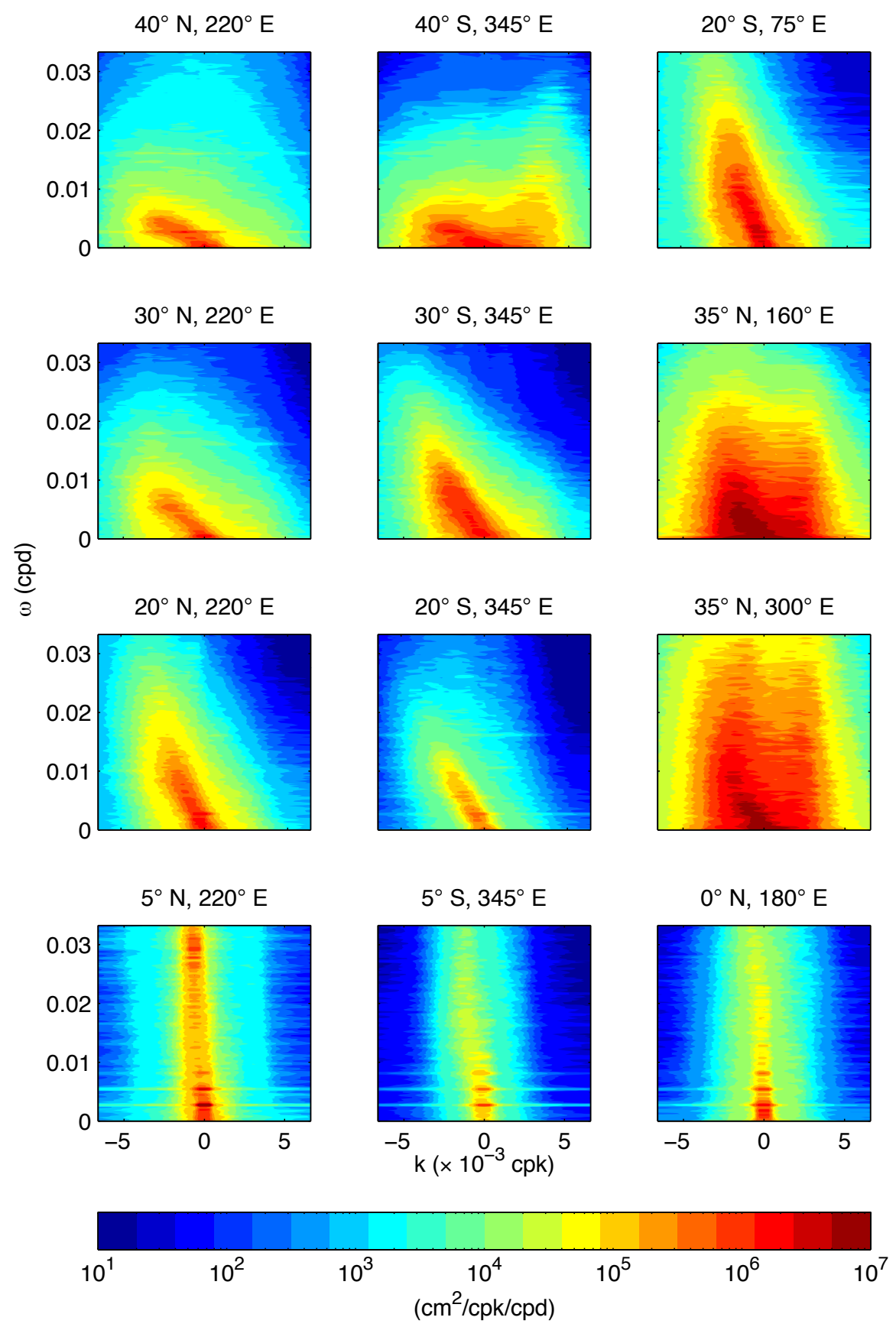

Figure 2-16: Two-dimensional zonal wavenumber-frequency spectrum for the region indicated at the top of each panel. The left panels show the North Pacific; the center panels shows the South Atlantic; the right panels show Indian Ocean, Kurohsio, Gulf Stream, and equatorial regions. The dominant phase speed is westward in all regions and increases toward the equator. 
by a quasi-universal frequency-wavenumber spectrum with characteristics described below.

a) A dominant ridge of westward variance. The zonal wavenumber-frequency spectrum is dominated by a ridge of westward variance. The slope of the ridge, in $k$ - $\omega$ space, decreases with latitude and corresponds to the dominant zonal phase speed.

b) Dominant phase speed is independent of wavelength. The ridge of westward variance follows a straight line in $k$ - $\omega$ space, so that the zonal phase speed is independent of $k$.

c) Strong westward variance extends beyond the baroclinic Rossby wave cutoff. At a given latitude there is a maximum frequency for baroclinic Rossby waves, $\omega_{\max }$. Hence, the classic theory of baroclinic Rossby waves can only account for excess westward variance at frequencies below $\omega_{\max }$. However, westward variance dominates the spectrum at higher frequencies as well. This may be due to the presence of barotropic waves, which have much higher phase speeds, extended Rossby wave theories that increase $\omega_{\max }$ [such as Killworth and Blundell, 2005; Tailleux and McWilliams, 2001], or nonlinear mesoscale vortices as suggested by Chelton et al. [2007, 2011]. These theories will be discussed further in Ch. 4.

d) Range of meridional propagation directions. The meridional wavenumber spectrum is nearly isotropic, in the sense that meridional propagation is nearly balanced between northward and southward. As a consequence of the spread in meridional propagation direction, the ridge of westward variance is elongated in the meridional wavenumber direction relative to the zonal wavenumber direction.

e) Spectrum is broad-band. The spectrum is a broad continuum in space and time, with no clear spectral peaks. A peak at the annual period is the exception, but it is suppressed here by removing the spatial mean at every time step before computing the spectrum. Since much of the annual period signal is large scale, 
removing the spatial mean attenuates the spectral peak that would otherwise exist.

\subsection{Global results}

The results in the previous section are typical, but the detailed shape of the spectrum changes geographically. Since repeating the analysis for each region in detail is not feasible, we map a few characteristic quantities on a $5^{\circ} \times 5^{\circ}$ grid (Fig. 2-17). Each grid point is based on the spectrum of the surrounding $30^{\circ} \times 10^{\circ}$ box. Note that these maps pick out one wavelength, period, or phase speed and form an incomplete picture of the full three-dimensional spectrum. However, the maps give a general sense of the geographic changes in the shape of the three-dimensional SSH spectrum.

We begin with the dominant zonal phase speed based on the Radon transform of the longitude-time plot. Zonal phase speed is generally westward, and increases toward the equator. Increasing phase speed corresponds to a steepening of the ridge of westward variance in the zonal wavenumber-frequency spectrum. The increase in phase speed from east to west across a basin is due to the increase in the baroclinic Rossby radius, owing to changes in stratification [Chelton et al., 1998].

The two exceptions to westward phase speed are along the equator and in the Antarctic Circumpolar Current, where eastward phase speed dominates. In the ACC, the strong eastward mean flow advects eddies eastward [Fu, 2009]. Along the equator, the eastward phase speed is due to fast moving Kelvin waves [Farrar, 2008]. For a more detailed discussion, see $F u$ [2009], who used a cross-correlation method to estimate propagation speeds with similar results.

Another useful way to characterize the model spectrum is in terms of its moments. 
Define

$$
\begin{aligned}
\left\langle\omega^{q}\right\rangle & =\int_{0}^{\infty} \omega^{q} \Phi_{\eta}(\omega) \mathrm{d} \omega / \int_{0}^{\infty} \Phi_{\eta}(\omega) \mathrm{d} \omega \\
\left\langle k^{q}\right\rangle & =\int_{0}^{\infty} k^{q}\left[\Phi_{\eta}(k)+\Phi_{\eta}(-k)\right] \mathrm{d} k / \int_{0}^{\infty}\left[\Phi_{\eta}(k)+\Phi_{\eta}(-k)\right] \mathrm{d} k \\
\left\langle l^{q}\right\rangle & =\int_{0}^{\infty} l^{q}\left[\Phi_{\eta}(l)+\Phi_{\eta}(-l)\right] \mathrm{d} l / \int_{0}^{\infty}\left[\Phi_{\eta}(l)+\Phi_{\eta}(-l)\right] \mathrm{d} l
\end{aligned}
$$

for integer $q$. For $k$ and $l$, we consider the moments of the spectrum averaged over positive and negative wavenumber ${ }^{5}$. Maps of the inverse of the first moments of frequency and wavenumber are shown (Fig. 2-17). Hatched areas in the wavelength plots indicate regions where there is more power in the positive wavenumber part of the spectrum than in the negative wavenumber part. For zonal wavenumber, hatching indicates a dominance of eastward propagation. For meridional wavenumber, hatching indicates a dominance of northward propagation.

The period generally decreases toward the equator, following the increase in phase speed. The minimum period occurs in the eastern tropical Pacific, primarily associated with the strong and relatively high frequency tropical instability wave and Kelvin wave activity. While the tropical instability wave signal is stronger north of the equator [Chelton et al., 2000], annual and lower frequency power is reduced south of the equator, resulting in the shortest time periods just south of the equator.

Dominant periods are quite different in the North Pacific and North Atlantic. In particular, the longest periods are seen in the northwest Pacific, while periods in the northeast Atlantic are relatively short. This is related to the different natures of the two western boundary currents. Both have strong high-frequency variability. However, the Kuroshio oscillates between strongly and weakly meandering states [Jayne et al., 2009]. This state transition introduces more low-frequency energy in the Kuroshio region, increasing the dominant period. The Gulf Stream, on the other hand, extends further north, resulting in more high frequency motion north of $40^{\circ} \mathrm{N}$.

The overall patterns in the first moments of frequency and wavenumber spectra

\footnotetext{
${ }^{5}$ Alternatively, we might calculate the moments for positive and negative wavenumber independently, as in Wunsch [2010].
} 
shown in Fig. 2-17 arise from the change in slope of the ridge of westward variance in the $k-\omega$ spectrum. The slope of the ridge increases towards the equator. As the slope becomes steeper, more power is concentrated at lower wavenumbers and the line extends to higher frequencies. The change in slope of the ridge itself is related to the change in deformation radius and wave propagation speed, though the wave propagation speed is significantly higher than that predicted by the classical Rossby wave theory [Chelton et al., 2007]. The smallest wavelengths are found in the North Atlantic where weak stratification results in a small deformation radius.

As expected from the phase speed plot, westward zonal wavenumber $(k<0)$ dominates everywhere except in the ACC and at the equator. The first moment of zonal wavenumber does not show the area of eastward propagation along the equator because the spectra are computed from boxes spanning $10^{\circ}$ of latitude, and the strong westward propagation just off the equator overpowers the eastward propagation on the equator in the average. Eastward propagation shows up on the equator in the phase speed plots, which are based on Radon transforms of the longitude-time plot at the center of each box.

The pattern of dominant meridional propagation direction is more complicated than zonal propagation direction. The dominant propagation direction is poleward ( $l>0$ in the northern hemisphere, $l<0$ in the southern hemisphere) from about $20^{\circ} \mathrm{S}$ to $20^{\circ} \mathrm{N}$ and generally equatorward at higher latitudes.

Recall that the objective mapping procedure alters the shape of the spectrum from altimetry. The mapping procedure significantly steepens the wavenumber spectra, and so we have not mapped spectral slopes here. Instead, the reader is referred to $X u$ and Fu [2012]. However, the differences between the first moments of the spectra from along-track $\mathrm{T} / \mathrm{P}$ data and from the AVISO gridded data product are found to be small, typically less than $10 \%$. 

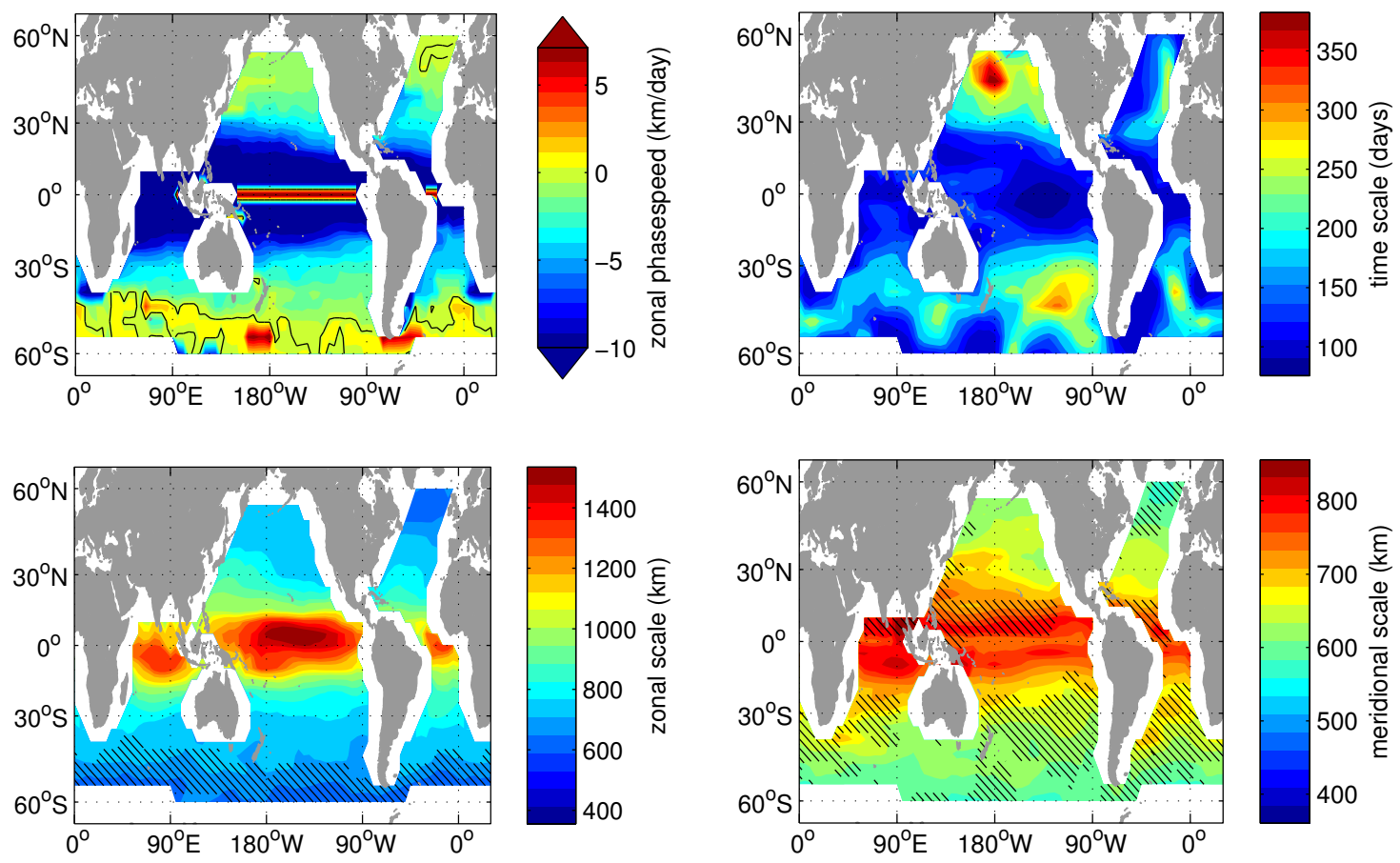

Figure 2-17: Dominant zonal phase speed as found in from a Radon transform of the longitude-time plot (top left). Dominant period (top right), zonal wavelength (bottom left), and meridional wavelength (bottom right) based on the inverse of the first moment of the one-dimensional spectra. For zonal wavelength, the hatched area indicates regions where eastward propagation dominates; elsewhere, westward propagation dominates. For meridional wavelength, the hatched area indicates regions where northward propagation dominates; elsewhere, southward propagation dominates. 


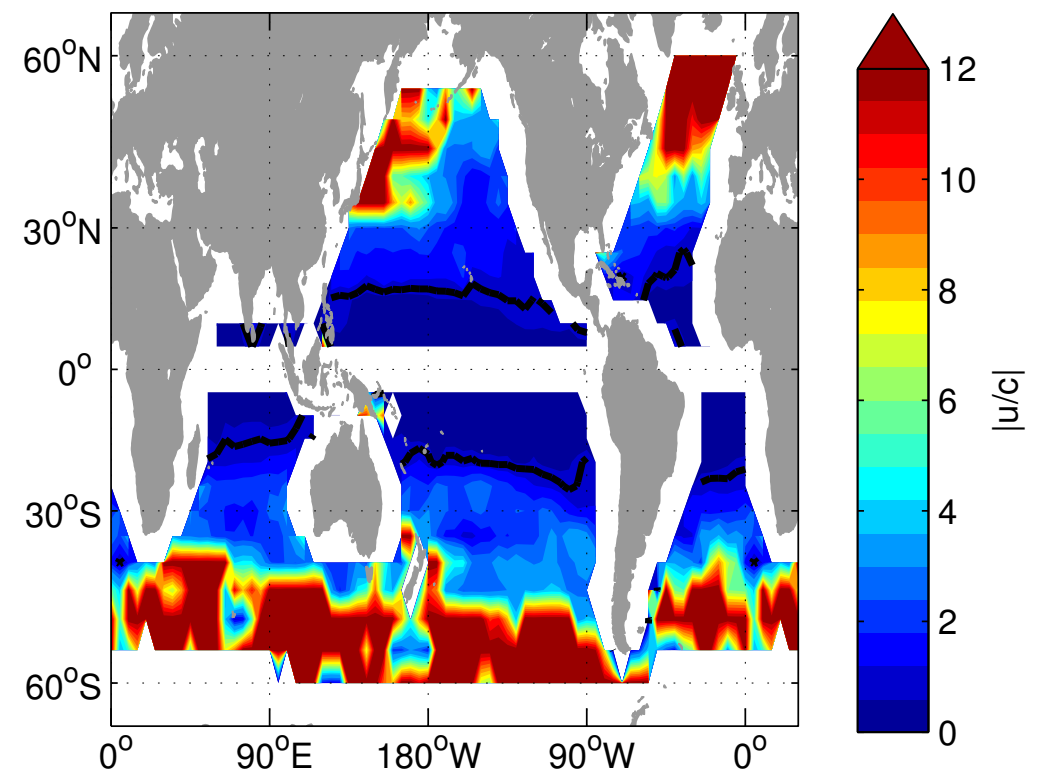

Figure 2-18: The nonlinearity parameter $U / c$ is small near the equator, but increases with latitude. The black contour shows $U / c=1$.

\subsection{Nonlinearity}

The nonlinearity of SSH variability is of interest, and can be assessed from the SSH fields. One estimate of nonlinearity is provided by the advective nonlinearity parameter $U / c$, where $U$ is the a fluid parcel speed and $c$ is the phase speed of SSH features. Values of $U / c \gg 1$ occur when fluid speeds are larger than SSH feature translation speeds, and imply that the SSH feature cannot be regarded as a linear wave disturbance propagating through a resting medium. Dynamics are nonlinear when the parameter $U / c$ exceeds 1 . Chelton et al. [2007, 2011] used the maximum rotational speed within identifiable vortices for $U$, and the vortex translation speed for $c$. Thus, they were estimating the nonlinearity of particular features. Here, we use the RMS velocity estimated from the AVISO altimetry for $U$, and dominant phase speed shown in Fig. 2-17 for $c$. Thus, we are estimating the nonlinearity of the SSH field, not of individual vortices. The nonlinearity parameter is less than 1 equatorward of about $20^{\circ}$ (Fig. 2-18). This result is generally consistent with earlier findings that high latitudes are generally more nonlinear than low latitudes [e.g. Fig. 17, Chelton et al., 2011]. 
From the spectrum, we can estimate the critical frequencies where nonlinearity becomes important. Specifically, we compare the wave period $\omega^{-1}$ to the advective turnover time $T$ due to wavelengths longer than $k^{-1}$. When the ratio of turnover time to wave period, $T \omega$, is less than one, nonlinear dynamics are important. The enstrophy at a particular frequency due to wavelengths longer than $k^{-1}$ is

$$
Z^{<}(k ; \omega)=\int_{0}^{k} k^{\prime 2} E\left(k^{\prime} ; \omega\right) \mathrm{d} k^{\prime}
$$

where

$$
E(k ; \omega)=\frac{g^{2}}{f_{0}^{2}} 4 \pi^{2} k^{2} \Phi_{\eta}^{N D}(k, \omega) \delta \omega
$$

is the energy spectrum at frequency $\omega$, and $\delta \omega$ is spacing between Fourier frequencies, and

$$
\Phi_{\eta}^{N D}(k, \omega)=\frac{\Phi_{\eta}(k, \omega)+\Phi_{\eta}(-k, \omega)}{2}
$$

is the non-directional SSH spectrum, averaged over positive and negative wavenumbers. The turnover time is

$$
T=\frac{1}{\sqrt{Z^{<}(k ; \omega)}}
$$

The spectral nonlinearity parameter $\mathrm{T} \omega$ at $30^{\circ} \mathrm{N}, 190^{\circ} \mathrm{E}$ is shown in Fig. 2-19. Nonlinearity is strongest at low frequencies and wavenumbers, and decreases towards high frequency and wavenumber. The transition from strong $(T \omega \ll 1)$ to weak $(T \omega \gg 1)$ nonlinearity occurs near 100 days., as shown by the black contour. We estimate the fraction of energy that is consistent with linear dynamics by integrating the kinetic energy spectrum $\Phi_{\mathrm{EKE}}(k, \omega)$ over the frequency-wavenumber range for which $T \omega>1$. At this latitude, roughly $75 \%$ of the kinetic energy is associated with scales where $T \omega<1$, confirming the expectation that nonlinear dynamics are important.

At lower latitudes $\left(10^{\circ} \mathrm{N}, 190^{\circ} \mathrm{E}\right)$, nonlinearity is important in a smaller region of spectral space, and more kinetic energy extends to scales with $T \omega>1$ (Fig. 2-20). At this latitude, only $25 \%$ of the kinetic energy is associated with scales where $T \omega<1$. 

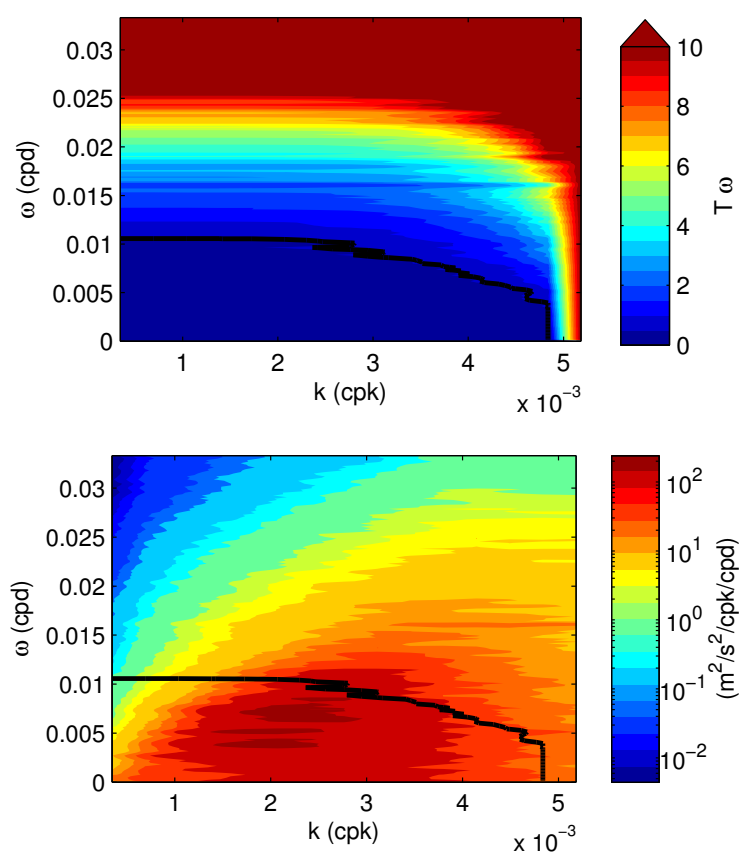

Figure 2-19: Nonlinearity as a function of $k$ and $\omega$ based on the ratio of the turnover time $T$ (2.16) to wave period $\omega^{-1}$ at $30^{\circ} \mathrm{N}, 190^{\circ} \mathrm{E}$ (top). The black contour shows $T \omega=$ 1. The kinetic energy spectrum is shown with $T \omega=1$ contour superimposed, and most of the kinetic energy is at frequencies where nonlinearity is important (bottom).
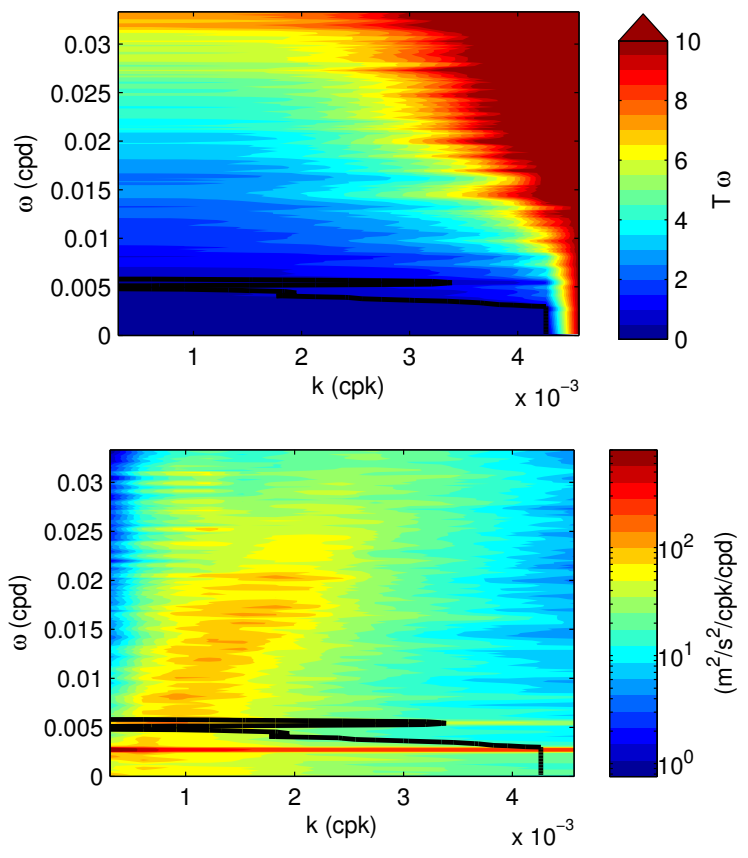

Figure 2-20: Same as Fig. $2-20$ but at $10^{\circ} \mathrm{N}, 190^{\circ} \mathrm{E}$ (top). The kinetic energy spectrum with $T \omega=1$ contour superimposed shows that much of the kinetic energy at this latitude extends to frequencies where nonlinearity is not significant (bottom). 


\subsection{Conclusions}

This chapter provides a broad description of the three-dimensional spectrum of sea surface height variability. The spectrum has a qualitatively universal shape over most

of the ocean interior (away from strong currents and the equator). In particular, the spectrum is dominated by a broad ridge of westward variance, consistent with the presence of planetary waves.

The spectrum varies geographically, and much of that variation is due to two causes. First, the deformation radius and Rossby wave phase speed vary strongly in the meridional direction and more weakly in the zonal direction. The variation in Rossby wave phase speed changes the slope of the ridge of westward variance and, consequently controls the dominant periods and wavelengths of variability. Mean flow, topography, and nonlinearity can all affect the dominant westward propagation speed, but the general pattern is clear and simple. The second major source of variation in the spectrum is the pattern of eddy kinetic energy over the ocean. Changes in eddy kinetic energy likely imply different dynamical regimes, and are closely linked to wavenumber spectral slopes [Xu and Fu, 2012].

The three-dimensional spectrum reveals that zonal and meridional wavenumber spectra are quite distinct. The zonal wavenumber spectrum is everywhere anisotropic, and westward propagation $(k<0)$ typically dominates. In contrast, the meridional wavenumber spectrum is nearly balanced between northward and southward propagation. The three-dimensional spectrum shows that SSH variability does not simply propagate westward, but has a significant meridional propagation as well, with strong wave propagation deviating by $60^{\circ}$ from zonal.

While use of the AVISO gridded altimetry has its advantages, it limits this study in several ways. In particular, the effective resolution of the gridded altimetry is about $2^{\circ}$ in space [Chelton et al., 2011] and 55 days. At shorter periods and wavelengths, the covariance function imposed by the objective mapping significantly attenuates the signal. As a result, we have not estimated high frequency and wavenumber spectral slopes which are of great importance for distinguishing between various turbulence 
theories.

Despite the limitations of the AVISO gridded altimetry, the gross features appear to be robust and the spectrum described here will serve as a useful backbone for the final model spectrum.

The observations outlined above suggest a number of important questions, which we only mention here.

a) What sets the dominant phase speed? The zonal phase speed is clearly related to the beta-effect, but typically exceeds the classic baroclinic Rossby wave phase speed by a factor of two. The excess phase speed may be due to mean flow effects [Killworth and Blundell, 2004, 2005], nonlinear vortex dynamics [Chelton et al., 2011], or other causes. In Ch. 4, we argue that the excess phase speed is due, at least in part, to the impact of rough bottom topography.

b) To what extent is the observed sea surface motion due to linear waves versus coherent nonlinear vortices? Consider a propagating coherent vortex, modeled as a Gaussian bump with mesoscale dimensions. Since the Fourier transform of a Gaussian is itself Gaussian, the power spectrum of the coherent vortex will have strong low frequency/wavenumber components. This spectrum is not easily distinguishable from the spectrum of a wave field with constant phase speed. The phase of the Fourier transform may provide a way to distinguish between the two. Spectral estimates of the nonlinearity suggest that, especially at high latitudes, most of the energy in the spectrum is associated with nonlinear dynamics.

c) What sets the wavenumber spectral slope observed by $X u$ and Fu [2012]? One possibility is that different turbulent regimes (QG vs. SQG) dominate in different geographical areas. On the other hand, the forcing and dissipation wavelengths may not be sufficiently separated to allow an inertial range. 


\section{Chapter 3}

\section{Vertical structure of variability}

This chapter is concerned with the vertical structure of ocean variability. Moored, drifting, and ship-based measurements provide direct observation of the variability of ocean currents, pressure, temperature, and salinity. However, these measurements are generally sparse in space and time. There is no equivalent of the satellite altimetric record for the sub-surface ocean. We use current meter records and numerical model output to describe the vertical structure of ocean variability. This will be combined with information about the spectrum of surface variability from Ch. 2 to create a model for the four-dimensional spectrum of ocean variability.

Wunsch [1997] analyzed the vertical partition of horizontal kinetic energy in a set of moored current meter records. For each mooring, the velocity profile was decomposed into classic free Rossby wave modes. While the dataset was only marginally suitable for the goal, the basic conclusion was that the vertical structure of kinetic energy was consistent with equipartition between the barotropic and first baroclinic modes, with decreasing energy in higher modes. There were signs that the vertical modes are coupled, so that the total energy is not equal to the sum of the energy in each mode. Wunsch [1997] also showed that the pattern of surface geostrophic kinetic energy extrapolated to the surface from current meters is generally consistent with surface kinetic energy from altimetry.

Zang and Wunsch [2001] proposed an empirical model of the four-dimensional spectrum of ocean variability. The horizontal wavenumber and frequency spectrum 
was based on altimetric observations, and the vertical structure essentially followed the scheme of Wunsch [1997]. Specifically, they proposed that the vertically integrated modal energies for the barotropic and first two baroclinic modes were in the ratio 1:1:1/2. They made no attempt to account for any modal coupling.

We analyze output from a data constrained GCM produced by the Estimating the Circulation and Climate of the Ocean Phase II (ECCO2) project to further study the vertical structure of ocean variability. How well do GCM's represent the vertical structure of ocean variability? Penduff et al. [2006] presented a comparison of the 1/6 CLIPPER Atlantic GCM with World Ocean Circulation Experiment (WOCE) current meter records. They developed metrics to quantify the differences between modeled and observed mean velocity and eddy kinetic energy (EKE). The model EKE tended to be too baroclinic; that is, energy was too weak at depth relative to the surface in the model. Scott et al. [2010] conducted a similar analysis, using a larger current meter database and evaluating four GCMs with $1 / 10^{\circ}-1 / 12^{\circ}$ resolution. All models had significant correlation between observed and modeled total kinetic energy (TKE), albeit with considerable scatter. More interesting than simple correlations were the model biases. The Ocean Circulation and Climate Advanced Modelling Project (OCCAM) and free-running HYbrid Coordinate Ocean Model (HYCOM) were biased toward weak TKE, especially below $1000 \mathrm{~m}$, similar to CLIPPER. In contrast, a data-assimilating $\mathrm{HYCOM}^{1}$ run and the Parallel Ocean Program (POP) model had much smaller bias relative to the current meter observations.

In Section 3.1, we describe the ECCO2 model used here, and in Section 3.2 we describe the Global Multi-Archive Current Meter Database (CMD) used to evaluate the vertical structure of kinetic energy in the model. The main goal of Section 3.3 is to evaluate the model representation of ocean variability, both at the surface relative to the altimetric observations, and sub-surface relative to current meter records. The main point of this chapter, in Section 3.4, is to describe the vertical structure of

\footnotetext{
${ }^{1}$ The data-assimilating HYCOM model assimilates sea surface height and sea surface temperature observations from satellites as well as in situ vertical profiles of temperature and salinity from XBTs, Argo floats, and moored buoys [Scott et al., 2010]. Current meter observations, which might force agreement, are not included in the assimilation.
} 
horizontal velocity and importance of mode coupling in the ECCO2 model.

\subsection{ECCO2 model description and processing}

The GCM results described here are based on output from the ECCO2 high-resolution ocean data synthesis [Menemenlis et al., 2008]. The ECCO2 data synthesis is the result of a least squares fit of the Massachusetts Institute of Technology general circulation model [MITgcm, Marshall et al., 1997] to available data. The model is run on a cubed-sphere grid [Adcroft et al., 2004]. This grid consists of six faces, each with 510 by 510 grid cells and a mean grid spacing of $18 \mathrm{~km}$. The model has 50 vertical levels, with thickness increasing from $10 \mathrm{~m}$ near the surface to $450 \mathrm{~m}$ at the bottom. The maximum depth is $6150 \mathrm{~m}$. The topography in the model is based on a blend of the Smith and Sandwell [1997] and General Bathymetric Charts of the Oceans one arc-minute bathymetric grid. The model time step is 20 minutes. Further details of the model configuration can be found in Menemenlis et al. [2008].

The ECCO2 data synthesis uses the Green function approach [Menemenlis et al., 2005] to constrain the MITgcm model to available satellite and in situ data. Control parameters, including atmospheric surface forcing, vertical diffusivity, vertical viscosity, drag coefficients, and initial conditions for temperature and salinity are adjusted to improve the fit of the model state to data in a least-squares sense. The data constraints include sea level anomaly from altimetry, time mean sea level, sea surface temperature from satellite observations, and temperature and salinity profiles from WOCE, TAO moorings, Argo floats, XBT's, etc. The resulting model is selfconsistent, in that it obeys the MITgcm time evolution equations and there are no unphysical jumps in the ocean state.

The model solution used here, known as "cube84", spans 1992-2007. However, the atmospheric forcing fields are only optimized through 2002. After 2002, the atmospheric forcing fields have not been adjusted, and the imposed precipitation field does not balance evaporation. This leads to a drop in SSH and rise in salinity after 2002. In this chapter, we use output for the period 1992-2002 only. 
The main model quantities of interest are velocity $(u, v)$, squared-velocity $\left(u^{2}, v^{2}\right)$, potential temperature referenced to the surface $(\theta)$, and salinity $(S)$. These threedimensional quantities are saved as monthly-means on the cubed-sphere grid, with velocity components perpendicular to the grid faces. Model SSH is saved as a dailymean on the cubed-sphere grid. Before analysis, velocities are rotated to east and north components and linearly interpolated to a $1 / 4^{\circ} \times 1 / 4^{\circ}$ grid, while scalar fields are simply interpolated. Before computing frequency spectra from ECCO2 SSH, the daily output is averaged over 7 days to achieve the same time resolution as AVISO.

\subsection{Current meter record processing}

The vertical structure of kinetic energy in the ECCO2 model will be compared to current meter records in an updated version of the CMD described by Scott et al. [2010]. The CMD includes records from several sources, including the National Oceanic Data Center, Fisheries and Oceans Canada, Oregon State University's Buoy Group, French Research Institute for Exploration of the Sea, and records collected by Carl Wunsch. In all, the CMD contains 45,238 current meter records collected between 1960 and 2009, though some are duplicates. These records were subject to various quality control measures, either in the original archive or in the compilation of the CMD. These measures include checks for stalled rotors, erroneous depth information, and redundant records, as described by Scott et al. [2010]. No attempt was made to correct for mooring blow-over.

For this study, we only consider records with a duration of at least 180 days in water depth of at least $3000 \mathrm{~m}$ and instruments more than $500 \mathrm{~m}$ above the sea floor. After removing redundant records, 5229 records at 1068 mooring locations meet the criteria. Of these, records with less that $50 \%$ data coverage are excluded, leaving 4720 records at 1035 mooring locations for analysis. Fig. 3-1 shows the locations of the moorings analyzed.

The sampling frequencies for the records analyzed vary from 5 min to 1 day, so that many records contain tidal and internal wave signals. For comparison with the 


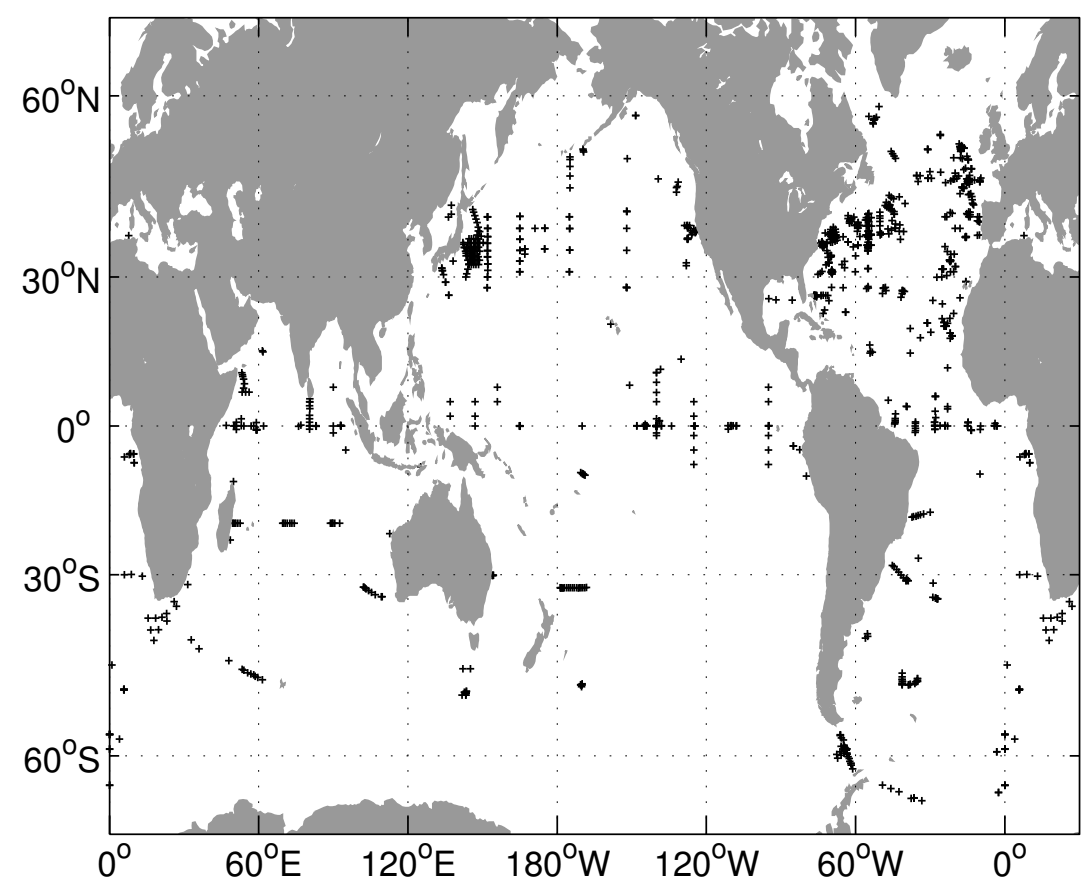

Figure 3-1: Locations of the 1068 mooring sites with records longer than 180 days and in water deeper than $3000 \mathrm{~m}$.

GCM output, which lacks both tides and internal waves, all current meter records are reduced to 5 day averages. When there are missing data within a 5 day window, the average is computed as long as there are at least 2.5 days of good data within the window; otherwise, the time period is flagged as missing.

\subsection{ECCO2 model evaluation}

The main objective of this section is to present an overview of the characteristics of the ECCO2 model. We compare the mean and variance of $\mathrm{SSH}$ with altimetric observations, and the vertical structure of TKE with current meter observations. A more detailed analysis of the vertical structure of variability in the model is made in the following section.

Before comparing the ECCO2 model to altimetry, we must point out that the altimetry itself has some errors and uncertainties. One source of error, noted in Ch. 2, is the AVISO objective mapping procedure. The mapping procedure imposes a spatial 
and temporal covariance function which impacts the shape of the spectrum and the total variance. Instrument noise (atmospheric corrections, sea state bias, orbit errors) and "representation errors" (residual tides, inverted barometer response to pressure loading) also lead to time-variable errors. Instrument noise has been estimated from differences between nearly-simultaneous measurements by two satellite altimeters. These estimates give an rms error of about $3 \mathrm{~cm}$, but the error associated with unresolved SSH features may be much larger [Ponte et al., 2007]. There are also errors in the mean dynamic topography (MDT) estimate. We use the CNES-CLS09 MDT. While errors in the MDT are difficult to estimate, formal error estimates are largest in the western boundary currents, at about 4-5 cm [Rio et al., 2011]. They also showed differences between the CNES-CLS09 MDT and an earlier MDT [Rio et al., 2005]. The differences between the two were as large as $30 \mathrm{~cm}$ near the Kuroshio, giving a rough estimate of the errors in MDT. Poorly understood errors related to the geoid may be larger. Still, comparison with altimetry will provide useful insight into the performance of the ECCO2 model.

\subsubsection{Quasi-steady state diagnostics and general circulation}

Time series of volume-averaged TKE and EKE, salinity, and potential temperature, as well as area-averaged SSH are shown in Fig. 3-2. In the first year, the TKE rises to its mean value of $28 \mathrm{~cm}^{2} / \mathrm{s}^{2}$ and fluctuates around that value. Note that the mean SSH begins to drop after 2002, when the un-optimized rain field is used, causing the salinity to rise. While the model kinetic energy does not appear to be affected by the imbalance, we limit analysis to the period 1992-2002.

The ECCO2 mean dynamic topography (MDT) field has a similar general structure and amplitude to the altimetric (MDT), and the two fields have a significant spatial correlation of $r=0.9, P<0.001$. (Fig. 3-3). However, there are significant differences, especially in the western boundary current regions. The map of the difference, AVISO MDT minus ECCO2 MDT, shows a low-high-low tripole pattern just off Cape Hatteras, indicating that the model Gulf Stream is more spread out than the altimetric Gulf Stream. Also, the model Kuroshio exhibits a strong and persis- 

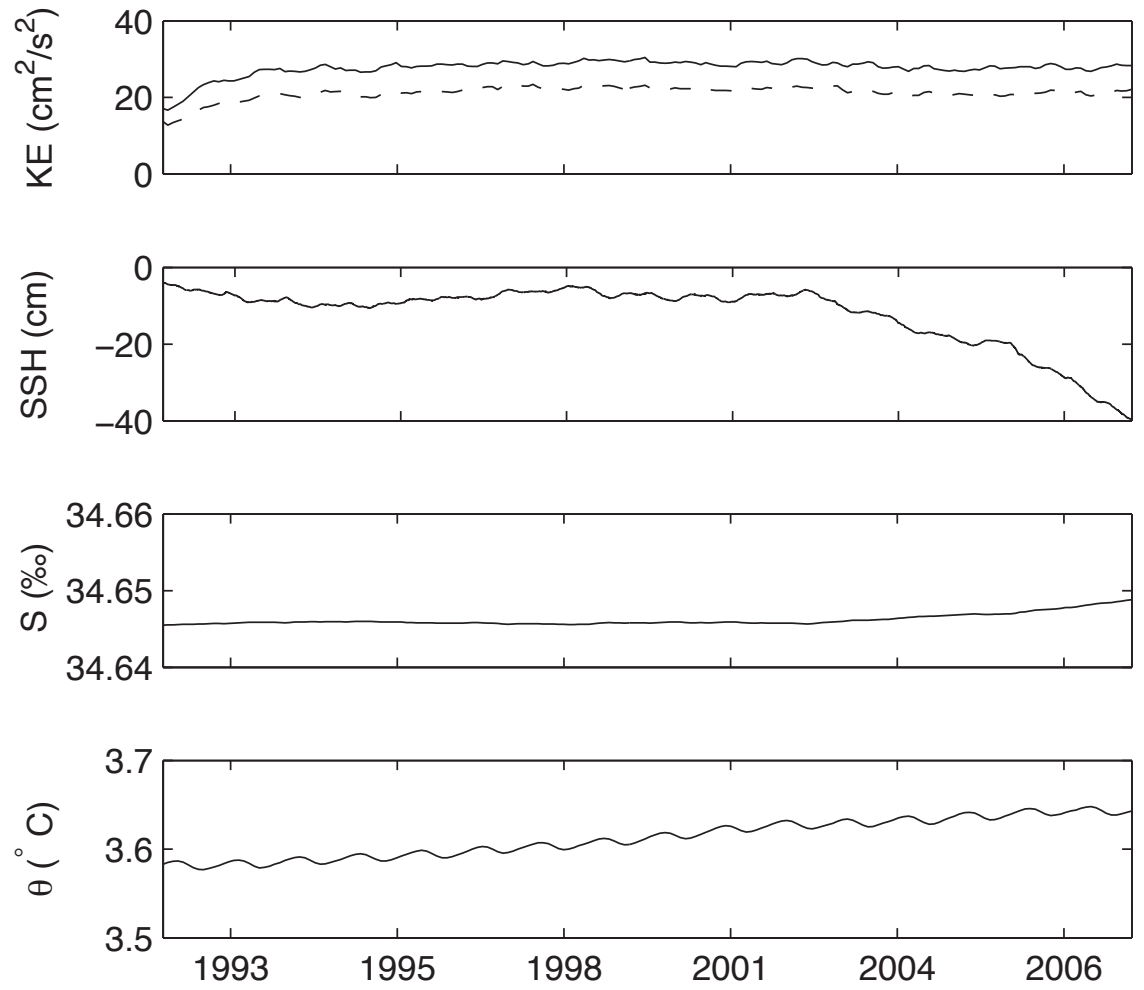

Figure 3-2: Global-mean total (solid) and eddy (dashed) kinetic energy (top), SSH, salinity, and potential temperature for the ECCO2 model. An imbalance in the unoptimized rain field imposed after 2002 results in a drop in SSH and rise in salinity. Pre-2002 salinity variations are very small; less than $0.001 \%$. Annual temperature variations are about $0.01^{\circ} \mathrm{C}$ with a warming trend of $0.004^{\circ} \mathrm{C} /$ year. 
tent meander along the coast of Japan, just south of the separation point, which is not seen in observations. Both the Kuroshio and Gulf Stream recirculation flows are weaker in the model than in altimetric observations.

The variance of SSH in the ECCO2 model is again broadly similar to altimetric observations, with a relatively weak but statistically significant spatial correlation of $r=0.4, P<0.01$ (Fig. 3-3). As with the MDT, much of the difference between the model and altimetric SSH variance lies in the major currents. First, in the Gulf Stream, altimetry shows the highest variance region beginning at Cape Hatteras and extending primarily eastward before turning northward near $310^{\circ} \mathrm{E}$. In ECCO2, the region of highest variance near the Gulf Stream is displaced south of Cape Hatteras. This shift in the region of strongest SSH variance shows up as a dipole in the difference map. Second, the observed Kuroshio extension continues further into the North Pacific than does the ECCO2 Kuroshio extension. Additionally, altimetry shows regions of very low SSH variance in the South Atlantic and Pacific, with no comparably low values in the model ocean; the lowest variance in the ECCO2 South Atlantic is a factor of three higher than the lowest variance seen by altimetry in the South Atlantic.

The path of Agulhas rings in the model is quite unrealistic. After separating from the from the coast, model Agulhas rings follow a very consistent path northwest to about $25^{\circ} \mathrm{S}$ before turning more westward and often continuing across the whole Atlantic. This consistent track leaves a streak of elevated SSH variance, while observations show that the path of Agulhas rings is much more variable. On the positive side, the ECCO2 model reproduces significant variability associated with the Azores Current around $35^{\circ} \mathrm{N}$ in the Atlantic, which is absent from some higher resolution models [e.g. 1/10 POP, Maltrud and McClean, 2005].

Our primary interest is in variability in the ocean interior, away from the core of the major ocean currents. Given this focus, the shift in the core of the Gulf Stream and Kuroshio are not major sources of concern. Errors in the model Gulf Stream and Kuroshio extension (after the currents separate from the coast) are more troubling. Maps of SSH variance suggest the majority of the variance in the middle 

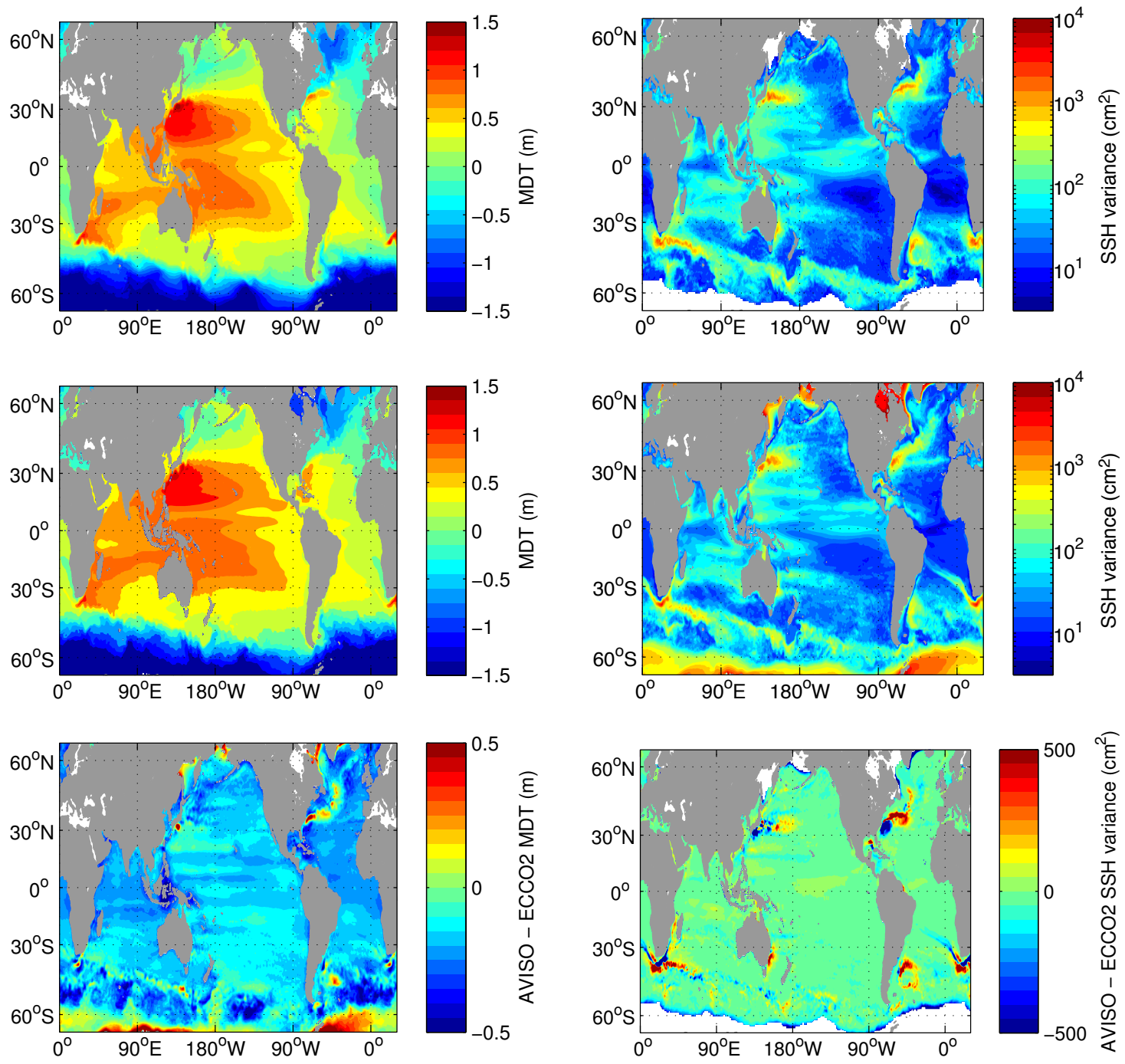

Figure 3-3: Mean dynamic topography (left) and SSH variance (right) from AVISO (top) and ECCO2 model (middle). Bottom row shows AVISO MDT minus ECCO2 MDT (left) and AVISO SSH variance minus ECCO2 SSH variance (right). For AVISO $\mathrm{SSH}$ variance, no value is shown if there are gaps in the record due to ice. Both fields have statistically significant spatial correlation between altimetric and model maps. The largest differences are in the position and sharpness of the major currents. See text for further discussion. 
of the North Atlantic and Pacific is associated with the boundary current extensions. Since the ECCO2 boundary current extensions do not continue as far into the basin as observations suggest, we anticipate that the model will have trouble reproducing observed variability there.

\subsubsection{ECCO2 SSH spectrum}

In Ch. 2, we describe several features of the two-dimensional zonal wavenumberfrequency spectrum of SSH. In particular, the spectrum is dominated by a strong ridge of westward variance with a slope, in $k$ - $\omega$ space, corresponding to the dominant phase speed. The slope and amplitude of the ridge of westward variance is closely related to the dominant period and wavelength of motion, and to the shape of the one-dimensional frequency and wavenumber spectra.

The ECCO2 model does capture some of the broad features of the observed spectrum (Fig. 3-4). In a low energy region (central North Pacific, $25^{\circ}-30^{\circ} \mathrm{N}, 190^{\circ}-230^{\circ} \mathrm{E}$ ), the model reproduces the narrow ridge of westward variance seen in the AVISO spectrum. The model also produces the same dominant phase speed (3.9 km/day) as the altimetry. However, beyond these qualitative similarities, the spectra are quantitatively very different. Relative to AVISO, the ECCO2 spectrum is weak by a factor of 10 along the ridge of westward variance, and too strong by a factor of 10 in the high frequency, positive wavenumber part of the spectrum corresponding to eastward propagation. The difference in frequency-wavenumber spectra here suggests that different dynamics are responsibile for generating the variability in the model and real ocean.

In a high energy region (Kuroshio extension, $38^{\circ}-45^{\circ} \mathrm{N}, 150^{\circ}-165^{\circ} \mathrm{E}$ ), westward motion still dominates, but eastward motions are stronger and the ridge of westward variance is less pronounced than in low energy regions. Both of these changes are qualitatively reproduced in the ECCO2 model. The amplitudes of the two spectra

agree within a factor of two over most of spectral space, with the exception of the high frequency-wavenumber corners.

These results show that a GCM can have a reasonable distribution of SSH variance 

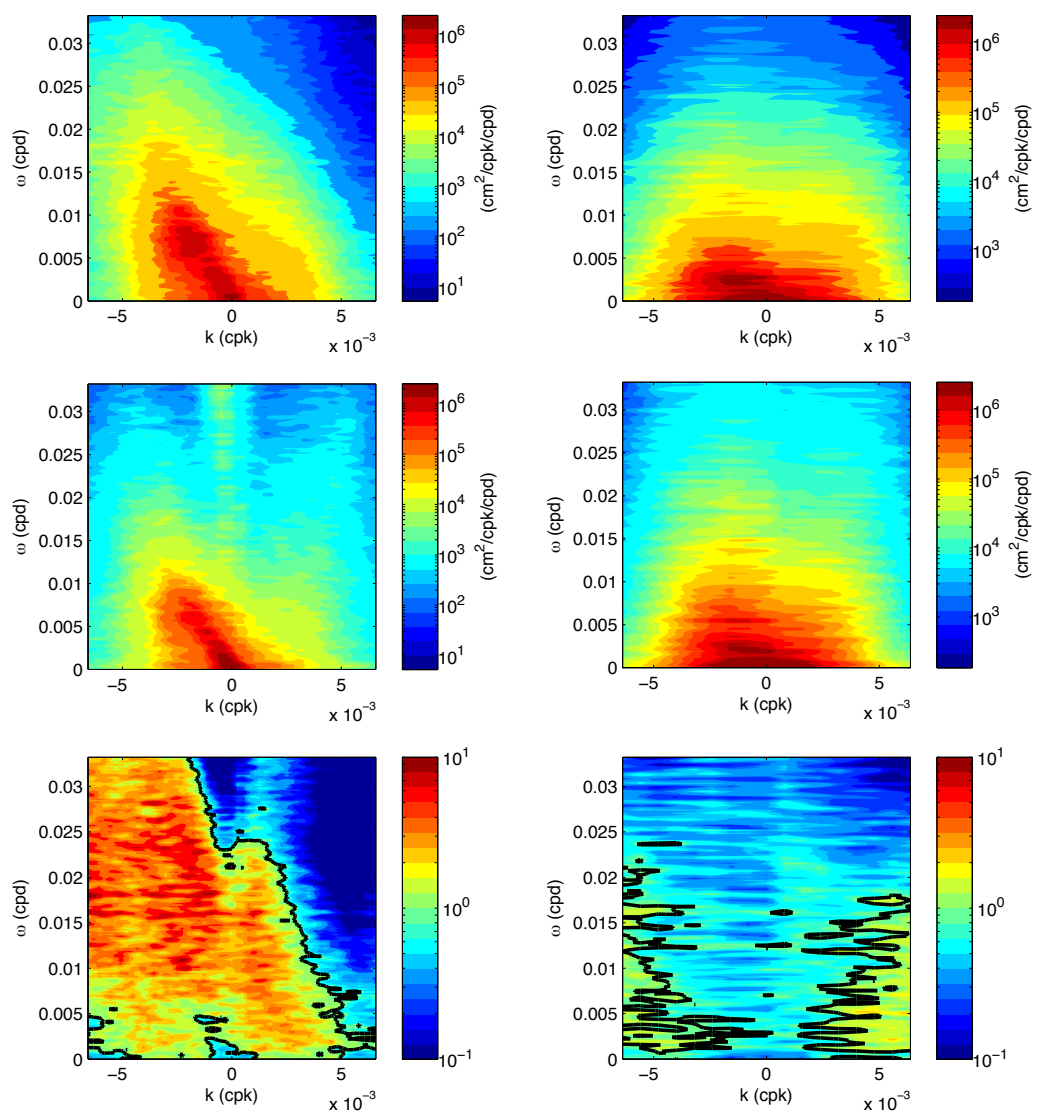

Figure 3-4: Comparison of zonal wavenumber-frequency spectra of SSH from a low energy region $\left(25^{\circ}-30^{\circ} \mathrm{N}, 190^{\circ}-230^{\circ} \mathrm{E}\right.$; left) and high energy region $\left(38^{\circ}-45^{\circ} \mathrm{N}, 150^{\circ}-\right.$ $165^{\circ} \mathrm{E}$; right) in observations and ECCO2. Spectra from AVISO are shown (top), along with similar spectra from ECCO2 (middle), and the ratio of AVISO to ECCO2 power (bottom). Note that the ratio is plotted with a logarithmic colorscale, and the black contour shows a ratio of unity.

in space (Fig. 3-3), but still fail to reproduce the observed space and periods of variability. In both the low- and high-energy discussed above, the SSH variance estimates for the model and altimetry agree to within $15 \%$. However, at the lowenergy region, the modeled and observed frequency-wavenumber spectra are very different. In particular, the ECCO2 spectrum has much lower amplitude along the ridge of westward variance (by a factor of 10 or so) and much higher amplitude for eastward propagating motions (again by a factor of 10). While the model's excess eastward variance may have as much to do with the processing of the gridded altimetry data as with errors in the model, the differences suggests that the model misses some important dynamical processes in the low-energy region. 


\subsubsection{Model vertical structure}

Does the ECCO2 GCM provide a reasonable representation of the vertical structure of kinetic energy? We compare the vertical structure of TKE averaged over all 1035 moorings found in the CMD to the vertical structure in ECCO2 averaged over the mooring locations (Fig. 3-5). For each low-pass filtered current meter record, we compute the time-mean total kinetic energy, $\overline{T K E}$. At each mooring location, $\overline{T K E}$ is averaged over all records within a depth bin, then over all moorings for each depth bin to form a vertical profile $\overline{T K E}(z)$. For the ECCO2 model, we compute the $\overline{T K E}$ for each mooring and each depth bin by averaging over all model grid points within $1 / 4^{\circ}$ radius of the mooring location. Then we average over all mooring locations in each depth bin to form a vertical profile of $\overline{T K E}(z)$ for the model. The averaging scheme is designed to give the same weight to high or low KE regions in the model as in the current meter records. The number of moorings with instruments in each depth bin is shown in the figure. For example, there are 218 moorings in the bin centered at $2500 \mathrm{~m}$.

The ECCO2 model $\overline{T K E}$ is similar to the current meter value in the upper ocean, but is systematically weak below. Above $300 \mathrm{~m}$, the two agree within $11 \%$ (average of $8.3 \times 10^{-2} \mathrm{~m}^{2} / \mathrm{s}^{2}$ for the CMD and $7.4 \times 10^{-2} \mathrm{~m}^{2} / \mathrm{s}^{2}$ for ECCO2) but below $2000 \mathrm{~m}$ the model has just over half the kinetic energy seen in the current meter oservations (average of $4 \times 10^{-3} \mathrm{~m}^{2} / \mathrm{s}^{2}$ for the CMD and $2.2 \times 10^{-2} \mathrm{~m}^{2} / \mathrm{s}^{2}$ for ECCO2). This result is consistent with the finding of Penduff et al. [2006] that modeled kinetic energy is too baroclinic.

The $\overline{T K E}$ profiles should not be over interpreted, since the different depth bins are averages over different latitudes and longitudes. The available current meter records are the result of many different experiments, and are not a representative sample of the ocean. Figure 3-5 also shows the $\overline{T K E}(z)$ profile averaged over the full model domain. The full domain average has roughly half the $\overline{T K E}$ of the model sampled at the mooring locations. Clearly there is a tendency for the current meter observations to be made in highly energetic regions such as the western boundary currents and the 


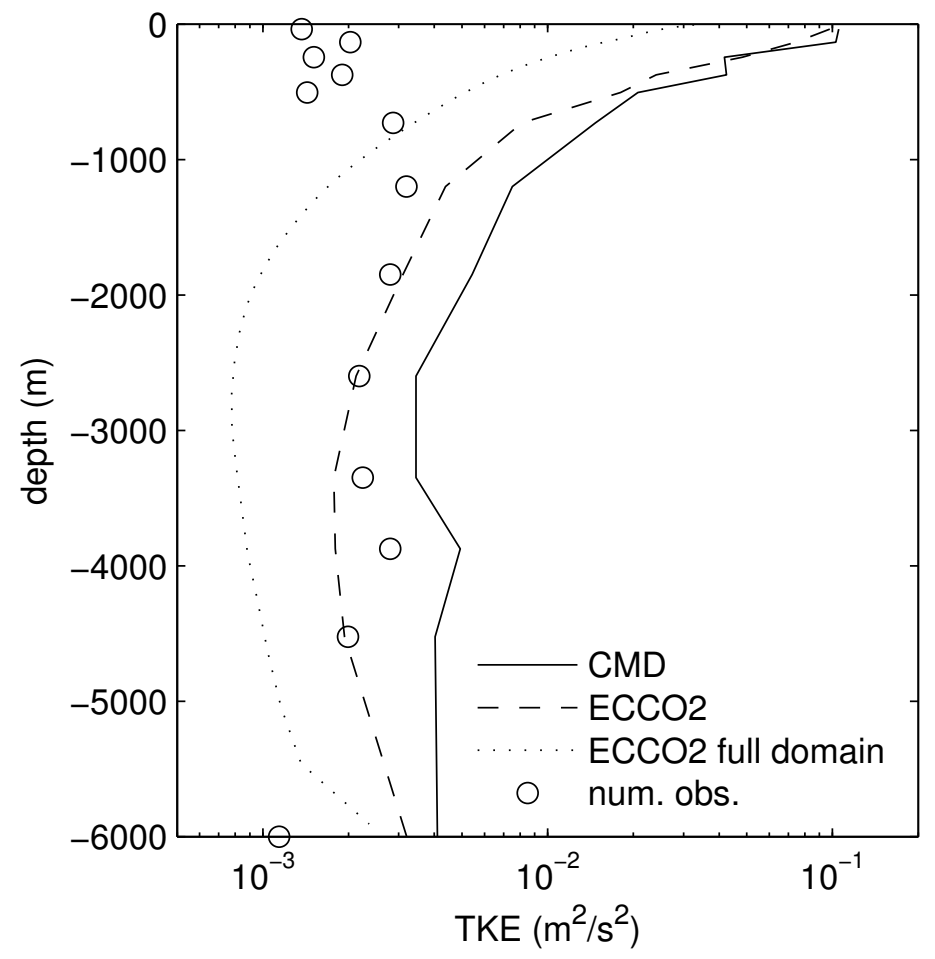

Figure 3-5: Vertical profiles of $\overline{T K E}$ averaged over all moorings (solid), over all mooring locations in ECCO2 (dashed), and over the full ECCO2 domain (dotted). The number of moorings in each depth bin (open circles) is $10^{5}$ times the number shown. 
equatorial ocean.

The ECCO2 model does not perform equally well in high and low energy regions. We repeat the above analysis twice, using only moorings from relatively energetic regions in one case and less energetic regions in the another (Fig. 3-6). We plot the same information in Fig. 3-7, now showing the $\overline{T K E}$ in each bin for ECCO2 divided by the corresponding value from the CMD. When only moorings in western boundary current regions, within $10^{\circ}$ of the equator, and in the ACC between $66^{\circ} \mathrm{S}$ and $55^{\circ} \mathrm{S}$ are included, the ECCO2 model captures about $80 \%$ of the observed $\overline{T K E}$ throughout the water column, performing only slightly better above $2500 \mathrm{~m}$ than below. When only moorings outside these regions are used, the ECCO2 model only captures about $45 \%$ of the observed $\overline{T K E}$ throughout the water column. When the mooring locations are divided into high and low energy regions, the $\overline{T K E}(z)$ profile does not appear too baroclinic on the whole. Rather, the model appears to perform better in high energy regions than in low energy regions. The apparent excess baroclinicity of $\overline{T K E}(z)$ in Fig. 3-5 is at least partially due to the way current meter records are distributed in the ocean.

We also wondered if the ECCO2 model performed better where the deformation radius is resolved than where it is not. Due to the distribution of current meters in the CMD, any effect of deformation radius is difficult to separate from the effect of kinetic energy level. We further divide the moorings in the low energy region (Fig. 3-6) into those poleward or equatorward of $30^{\circ 2}$. The model kinetic energy is slightly closer to the CMD kinetic energy equatorward of $30^{\circ}$ (average ratio 60\%) than poleward (average ratio 45\%). However, there are only 108 mooring locations in the low energy regions equatorward of $30^{\circ}$, as compared to 436 poleward and 1035 in the full analysis, and the uncertainty is likely high. Still, this result suggests that the model has more skill at low latitudes, where the deformation radius is fully resolved, than at high latitudes.

\footnotetext{
${ }^{2}$ The ECCO2 model grid spacing is about $18 \mathrm{~km}$, and nearly constant due to the cubed-sphere grid. At $30^{\circ}$, the deformation radius is about $40 \mathrm{~km}$ [Chelton et al., 1998], so that the model generally resolves the deformation radius equatorward of $30^{\circ}$ but not poleward.
} 

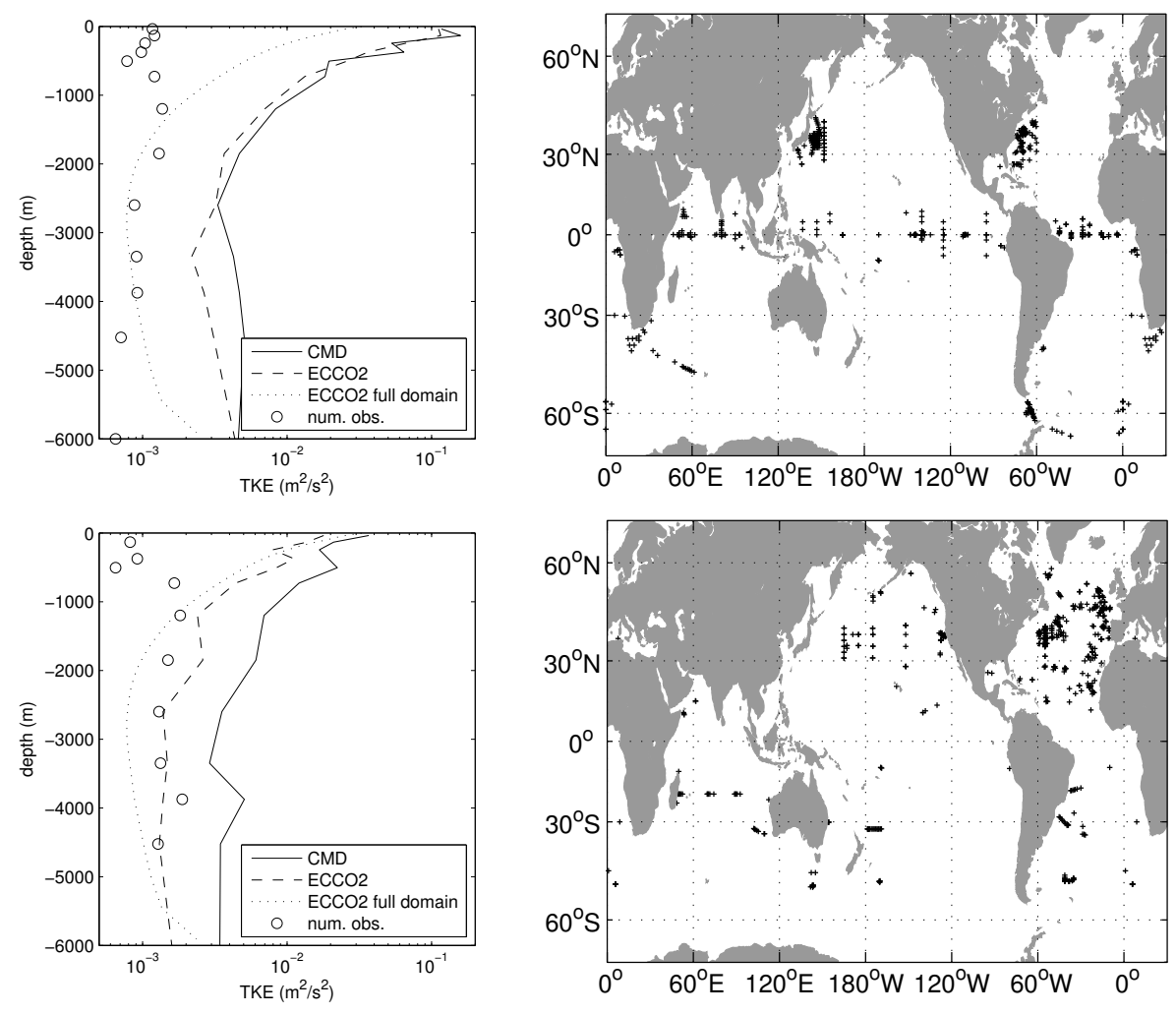

Figure 3-6: $\overline{T K E}(z)$ profiles as in Fig. 3-5, but divided into high energy regions (top) and low energy regions (bottom). $\overline{T K E}(z)$ profiles averaged over the full model domain are shown for reference (dotted line). Maps show the mooring locations used for each profile (right). 


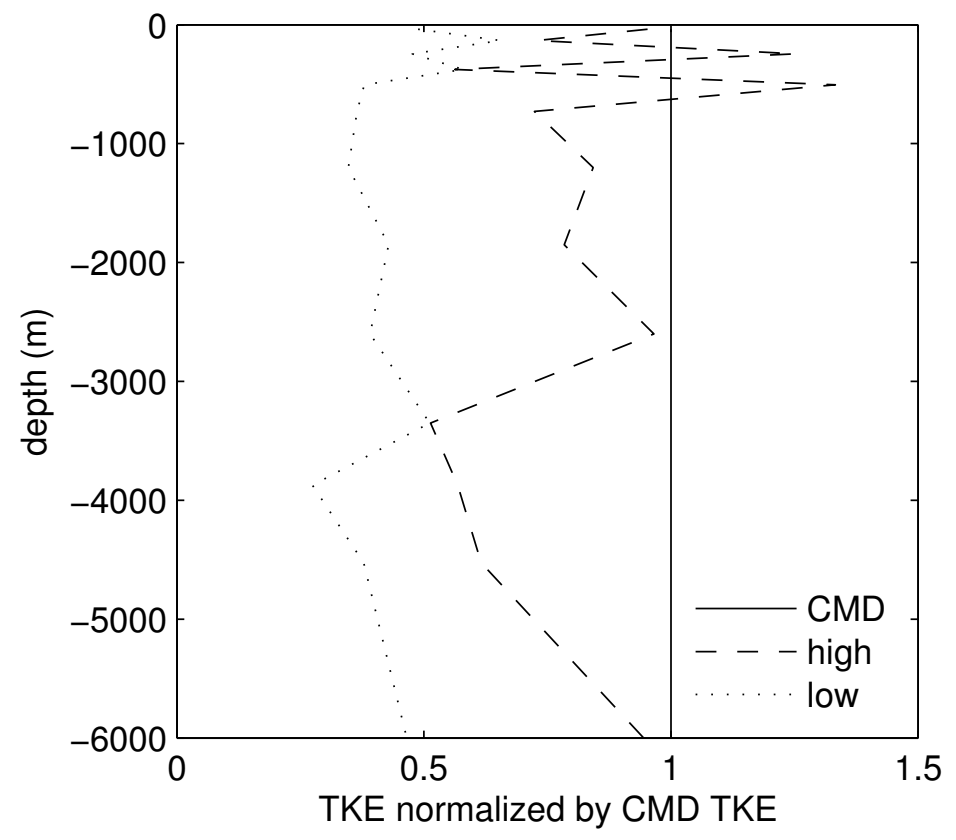

Figure 3-7: Vertical profiles of ECCO2 $\overline{T K E}$ normalized by CMD $\overline{T K E}$ for high energy region (dashed) and low energy region (dotted) as in Fig. 3-6. In the high energy region, the model $\overline{T K E}$ is about $83 \%$ of the CMD $\overline{T K E}$ averaged over the water column. In the low energy region, the average ratio is $45 \%$.

\subsubsection{Summary}

Above, we present a brief evaluation of 10 years of output from the ECCO2 model. Comparison with SSH spectra from altimetry and the vertical structure of kinetic energy from moored current meters both suggest that the model results are more realistic in high energy regions (including the western boundary currents, the ACC, and near the equator) than in the relatively quiescent ocean interior. Maps of MDT and SSH variability show that the model western boundary currents do not extend as far into the ocean interior as observations show, and this may limit the models ability to generate realistic variability in the mid ocean. Baroclinic instability is likely a major source of variability in the boundary current extension regions [Smith, 2007]. If the model's boundary current extensions are wrong, it won't have the same level of variability.

Our analysis of the vertical structure of kinetic energy is similar to that of Scott et al. [2010]. Our results suggest that the ECCO2 model performs comparably to 
the higher resolution OCCAM $\left(1 / 12^{\circ}\right)$ and free-running HYCOM $\left(1 / 12^{\circ}\right)$ models discussed in that study, but worse than the POP $\left(1 / 10^{\circ}\right)$ and data-assimilating HYCOM $\left(1 / 12^{\circ}\right)$ models (their Fig. 8). Penduff et al. [2006] concluded that resolution was the most important factor limiting the $1 / 6^{\circ}$ CLIPPER GCM in generating realistic kinetic energy. Scott et al. [2010] argued that there is no simple remedy for generating realistic kinetic energy, but suggested that model bottom friction also plays an important role. Here, we show that the ECCO2 model does not perform equally well in all places. However, diagnosing the causes of errors in the model is beyond the scope of our project.

\subsection{Vertical structure of currents in ECCO2 model}

Although there are significant problems with the ECCO2 model, especially the tooweak kinetic energy in relatively quiet regions of the ocean, the model does reproduce the overall surface variability and shape of the surface wavenumber-frequency spectrum from observations. Furthermore, ocean observations do not appear to be sufficient for a significantly more detailed analysis of the vertical structure of variability than that presented by Wunsch [1997]. In particular, current meter observations do not allow a more detailed analysis of the mode coupling that appeared in the current meter records. Given this, we now turn to a more detailed study of the vertical structure of kinetic energy in the ECCO2 model as a substitute until more comprehensive observations become available. The previous section compared the model with observations. Now, we consider how best to represent the vertical structure of KE in the model, in a statistical sense.

Our approach is to analyze the vertical structure of currents in the model in terms of the classic free, linear Rossby wave modes for a flat bottom, resting ocean. These modes satisfy

$$
\frac{\mathrm{d}}{\mathrm{d} z}\left(\frac{f_{0}^{2}}{N^{2}(z)} \frac{\mathrm{d} F}{\mathrm{~d} z}\right)+\gamma^{2} F(z)=0
$$


with rigid lid boundary conditions

$$
\frac{\mathrm{d} F}{\mathrm{~d} z}=0 \quad \text { at } \quad z=0,-H
$$

where $N(z)$ is the buoyancy frequency [Gill, 1982]. Equation (3.1) forms a SturmLiouville eigenvalue problem whose eigenvectors $F_{n}(z), 0 \leq n<\infty$, represent the vertical structure of horizontal velocity free modes in the ocean, and whose eigenvalues are related to the deformation radius $L_{d}=1 / \gamma$. The $n=0$ mode is barotropic, while the $n \geq 1$ modes are baroclinic with $n$ zero-crossings in the vertical. While the conditions under which these modes are derived (linear, flat bottom ocean with a resting mean state) are not valid in the real ocean, the modes form a complete set and they form a convenient basis for discussing the vertical structure of currents in the ocean.

The buoyancy frequency profile is estimated from time-mean temperature and salinity profiles from the ECCO2 model. We use the neutral density gradient method described by Chelton et al. [1998]. In this method, the buoyancy frequency is estimated by centered first differences of the potential density referenced to the midpoint between two depths,

$$
N^{2}\left(z_{m+1 / 2}\right)=-\frac{g}{\rho_{0}}\left(\frac{\rho\left(z_{m} \rightarrow z_{m+1 / 2}\right)-\rho\left(z_{m+1} \rightarrow z_{m+1 / 2}\right)}{z_{m}-z_{m+1}}\right)
$$

where $z_{m+1 / 2} \equiv\left(z_{m}+z_{m+1}\right) / 2$ is the midpoint between two model grid points and the notation $\rho\left(z_{m} \rightarrow z_{m+1 / 2}\right)$ is shorthand for the potential density of a water parcel at depth $z_{m}$ referenced to $z_{m+1 / 2}$. See Appendix A for further details. Typical vertical structures for the barotropic and first four baroclinic modes near $35^{\circ} \mathrm{N}, 220^{\circ} \mathrm{E}$ are shown in Fig. 3-8. 

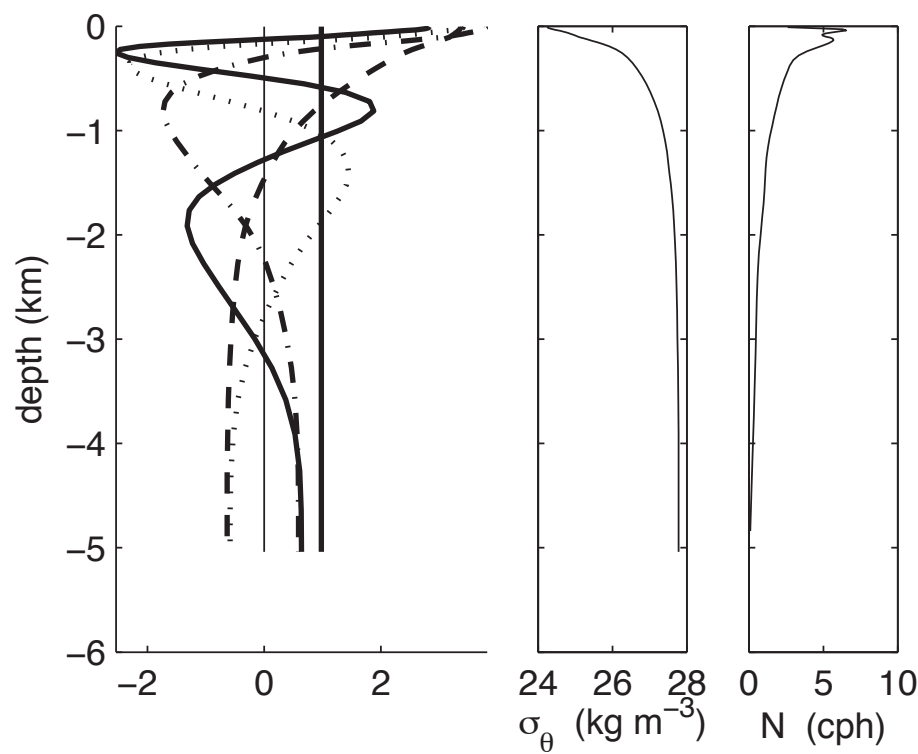

Figure 3-8: Barotropic and first four baroclinic horizontal velocity modes $F_{n}(z)$ near $35^{\circ} \mathrm{N}, 220^{\circ} \mathrm{E}$ (left) based on ECCO2 model potential density profile (center) and buoyancy frequency (right) at that location.

Model horizontal velocities may be represented as

$$
\begin{aligned}
& u(z, t)=\sum_{n=0}^{N} \alpha_{u, n}(t) F_{n}(z)+\varepsilon_{u}(z, t) \\
& v(z, t)=\sum_{n=0}^{N} \alpha_{v, n}(t) F_{n}(z)+\varepsilon_{v}(z, t)
\end{aligned}
$$

where the $\alpha_{u, n}(t)$ is the $n^{\text {th }}$ mode coefficient for zonal velocity $u, \varepsilon_{u}$ are noise residuals, and $N$ is the number of modes fit. We estimate the modal coefficients $\alpha_{u, n}(t)$ and $\alpha_{v, n}(t)$ based on a standard least squares fit of the first 20 modes to the model velocity at each time and location.

Typically, the barotropic and first baroclinic modes dominate the modal decomposition, with smaller contributions from higher modes. For example, near $35^{\circ} \mathrm{N}$, $220^{\circ} \mathrm{E}$ the RMS amplitudes of the barotropic and first four baroclinic modes are in the ratio $0.8: 1: 0.3: 0.2: 0.13$, similar to the a priori assumption of $1: 1: 1 / 2: 1 / 4: 1 / 8$ made by Wunsch [1997].

The vertical structure of horizontal kinetic energy can be reconstructed from the 
vertical modes. The mean kinetic energy is

$$
\overline{T K E}(z)=\frac{1}{2} \overline{\left(u^{2}+v^{2}\right)}=\frac{1}{2} \overline{\left[\left(\sum_{n=0}^{N} \alpha_{u, n}(t) F_{n}(z)\right)^{2}+\left(\sum_{n=0}^{N} \alpha_{v, n}(t) F_{n}(z)\right)^{2}\right]}
$$

where the overbar represents a time average. If the modes are temporally uncoupled, this reduces to the sum of the kinetic energy in each mode:

$$
\overline{T K E}_{\text {uncoupled }}(z)=\frac{1}{2} \sum_{n=0}^{N}\left({\overline{\alpha^{2}}}_{u, n} F_{n}^{2}(z)+{\overline{\alpha^{2}}}_{v, n} F_{n}^{2} z\right)
$$

We reconstruct the mean $\overline{T K E}(z)$ profile using the barotropic and first four baroclinic modes (Fig. 3-9). While including all 20 modes used in the decomposition improves the fit, it is remarkable that a few low modes captures most of the vertical structure of $\overline{T K E}(z)$. However, assuming that the modes are uncoupled underestimates the surface kinetic energy and overestimates it at depth. This simple test shows that linear, free, independent Rossby wave modes are not a complete description of model current variability. Either the modes are coupled, or some other dynamical modes are more appropriate.

The coupling of the modes can also be seen in longitude-time plots of the modal coefficients (Fig. 3-10). Individual features are seen to propagate together in both the barotropic component $\alpha_{u, 0}$ and first baroclinic component $\alpha_{u, 1}$ of zonal velocity. One possible explanation is that the coupled mode vertical structure is due to coherent mesoscale vortices, with equivalent-barotropic vertical structure [Killworth, 1992; Arbic and Flierl, 2003, 2004a,b]. However coherence persists to the lowest wavenumbers and frequencies; the mode coupling is not limited to the mesoscale features that stand out so prominently in the longitude-time plots (Fig. 3-11). Barotropic and first baroclinic modes are coherent over all frequencies, and in phase such that they reinforce at the surface and cancel at depth.

The results described above near $35^{\circ} \mathrm{N}, 220^{\circ} \mathrm{E}$ are quite general: the barotropic and first baroclinic modes have significant correlation almost everywhere (Fig. 3-12). We 


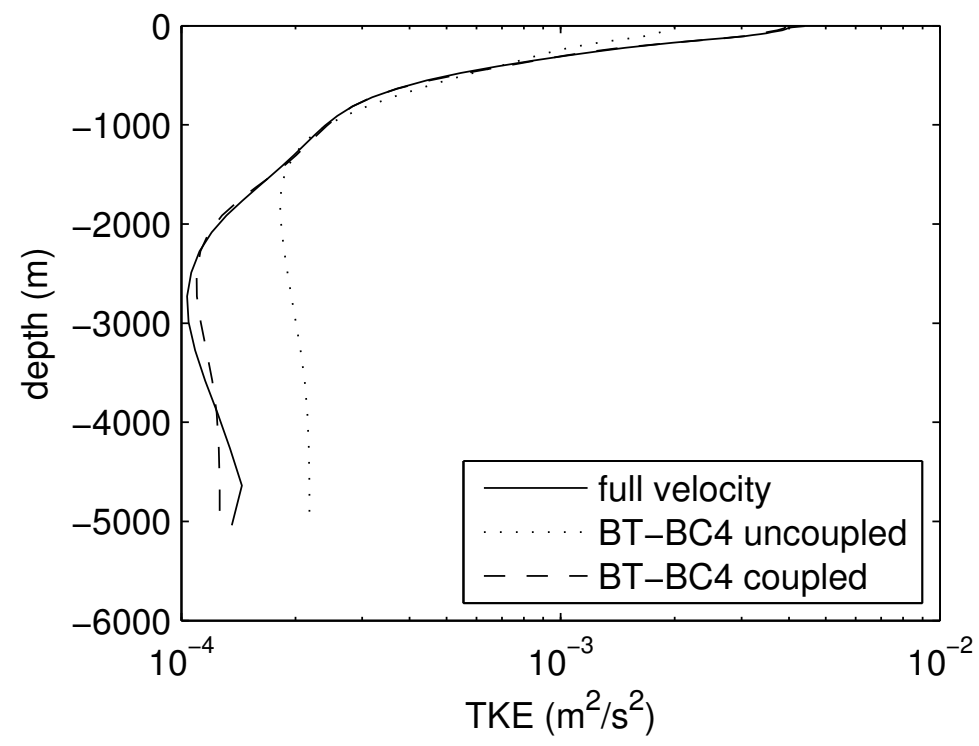

Figure 3-9: Vertical profile of $\overline{T K E}$ near $35^{\circ} \mathrm{N}, 220^{\circ} \mathrm{E}$ (solid) and reconstructed from the barotropic and first four baroclinic modes assuming no modal coupling (dotted) and with modal coupling (dashed). Without mode coupling, the modes cannot reconstruct the model $\overline{T K E}$ profile.
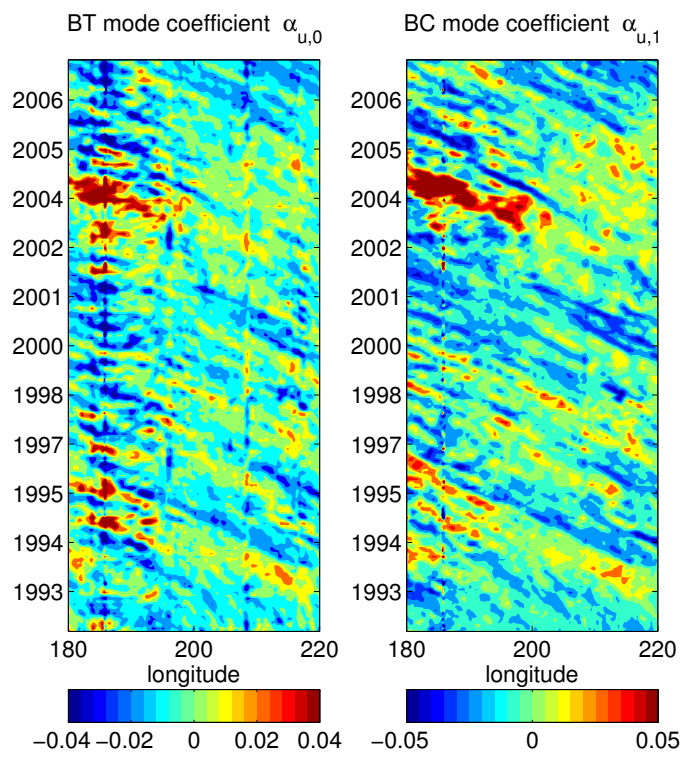

Figure 3-10: Longitude-time plots of barotropic mode coefficient (left) and first baroclinic mode coefficient (right) for zonal velocity show that features propagate together in the two modes, suggesting that the modes are coupled. The section shown here is at $35^{\circ} \mathrm{N}$ spanning $180^{\circ}-220^{\circ} \mathrm{E}$ 

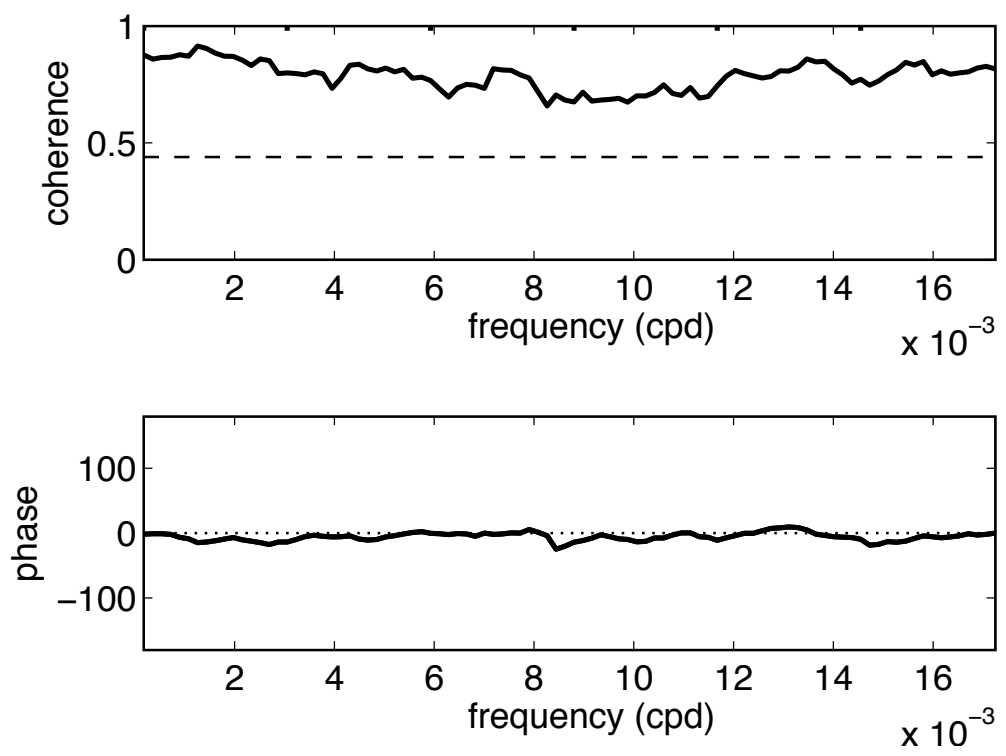

Figure 3-11: Temporal coherence (top) and phase (bottom) between the barotropic and first baroclinic mode coefficients near $35^{\circ} \mathrm{N}, 220^{\circ} \mathrm{E}$. The modes are coherent over all frequencies, and in phase such that they reinforce near the surface and cancel at depth. The $95 \%$ level of no significance is shown for coherence amplitude (dashed).

show the temporal correlation of the barotropic and first baroclinic mode coefficients for zonal velocity, $\alpha_{u, 0}$ and $\alpha_{u, 1}$, at each point. (Results with meridional velocity coefficients are indistinguishable.) The modal coefficients are approximately normally distributed, and significance levels are estimated using Student's t-test. Shaded areas indicate the $99 \%$ level of no significance, i.e. locations where the probability of finding the estimated correlation if no correlation exists is $P<0.01$. The only extended regions where the correlation is not significant at the $99 \%$ level are in the equatorial oceans, the Southern Ocean, and in the Northwest Pacific near the Sea of Okhotsk. Positive correlation, as with zero phase shift for coherence, implies that the modes reinforce near the surface and cancel at depth. There are only very limited regions of significant negative correlation.

The extensive correlation in Fig. 3-12 may appear surprising. Taken at face value, it suggests that there is some nonlinear dynamics coupling the barotropic and first baroclinic modes almost everywhere in the ocean. An alternative interpretation, which we pursue below, is that the classic flat-bottom Rossby wave modes are not the dynamically appropriate modes to use. If we can find alternative linear Rossby 


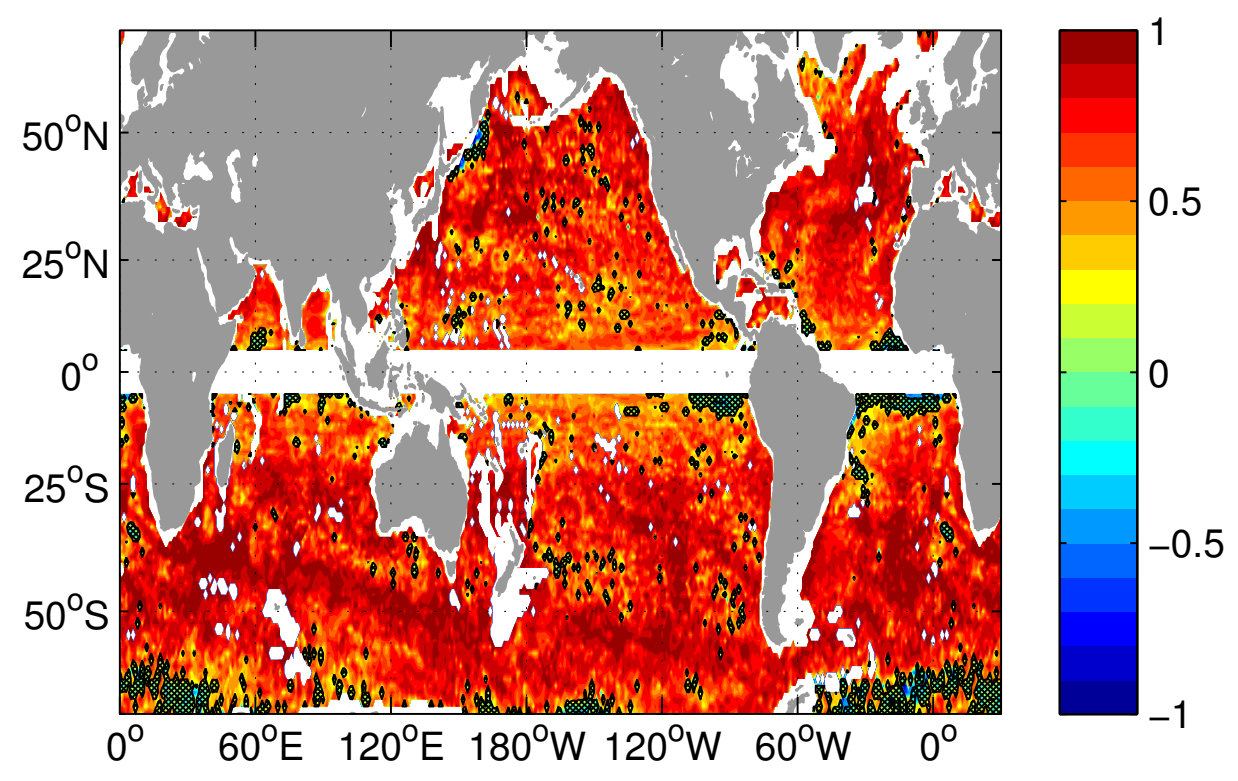

Figure 3-12: Correlation between the barotropic and first baroclinic mode coefficients is positive almost everywhere in the ocean. Regions where correlation is not significant at the $99 \%$ confidence level are indicated by hatching.

wave modes with vertical structure similar to the coupled barotropic and baroclinic mode shape, the ECCO2 model flow may still be consistent with linear Rossby waves.

\subsubsection{EOF analysis}

If the classic flat-bottom Rossby wave modes are not the dynamically "correct" modes for describing horizontal velocity in the ECCO2 model, in the sense that they require systematic coupling to describe the velocity, what do the correct modes look like? We now perform an empirical orthogonal function (EOF) analysis. At each location, we compute the EOFs of the horizontal velocity and then perform a least squares fit of the first 20 EOFs to the model velocity at each time. As with the classic Rossby wave modes, the result is a time series $\alpha_{u, n}(t)$ of coefficients for each EOF. Separate fits are performed for $u$ and $v$. Figure 3-13 shows the four leading EOFs for $u$. The leading EOF has a vertical structure similar to the typical first baroclinic mode shape, but offset so that it approaches zero at the bottom. This shape is consistent with the strong positive correlation between classic barotropic and first baroclinic Rossby wave modes seen above. 


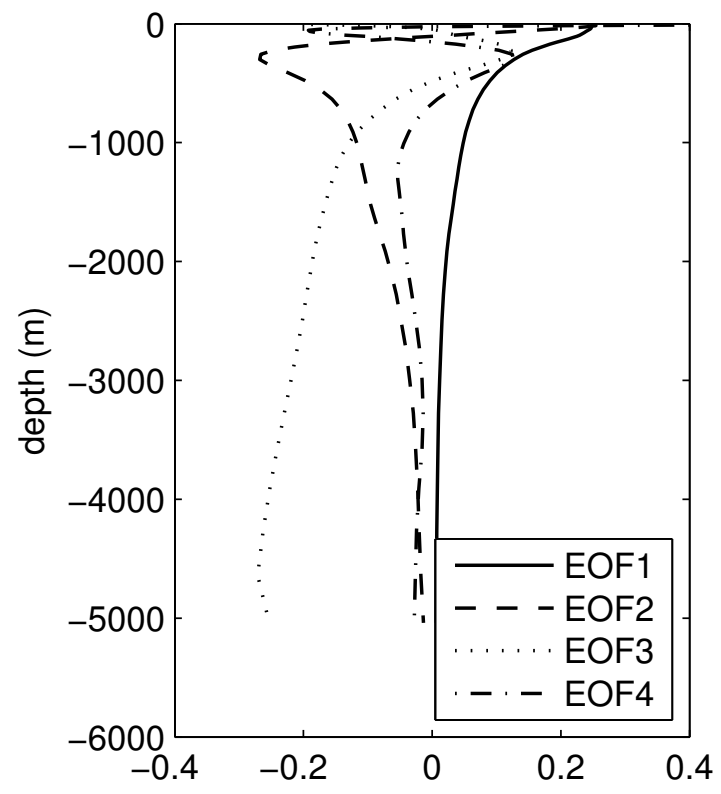

Figure 3-13: Four leading EOFs for $u$ near $35^{\circ} \mathrm{N}, 220^{\circ} \mathrm{E}$. These four EOFs account for $99 \%$ of the variance. The vertical structure of the first EOF is similar to the typical first baroclinic mode shape (Fig. 3-8), but offset so that it approaches zero at the bottom.

As with the classic Rossby wave modes, we reconstruct the mean kinetic energy profile $\overline{T K E}(z)$ from the time series of the EOF coefficients (Fig. 3-14). The kinetic energy is reconstructed from the first five EOFs. In contrast to the classic Rossby wave modes, the EOFs match the model $\overline{T K E}$ profile to within $10 \%$ whether coupling between the EOFs is taken into account or not. While the EOFs calculated here are not dynamical modes, they do give insight into the correct mode shape for describing the ECCO2 horizontal velocity.

\subsubsection{Frequency dependence of vertical structure}

One possible explanation for the nondispersive shape of the ridge of westward variance, hypothesized by Wunsch [2009], is that the ratio of energy in the barotropic and baroclinic modes changes along the nondispersive line. Based on Fig. 13 of Wunsch [2009], this hypothesis suggests that the mode ratio primarily depends on frequency. To test this hypothesis, we high-pass filter the ECCO2 velocity field with a cutoff period of 150 days and then perform the modal decomposition on the filtered velocity 


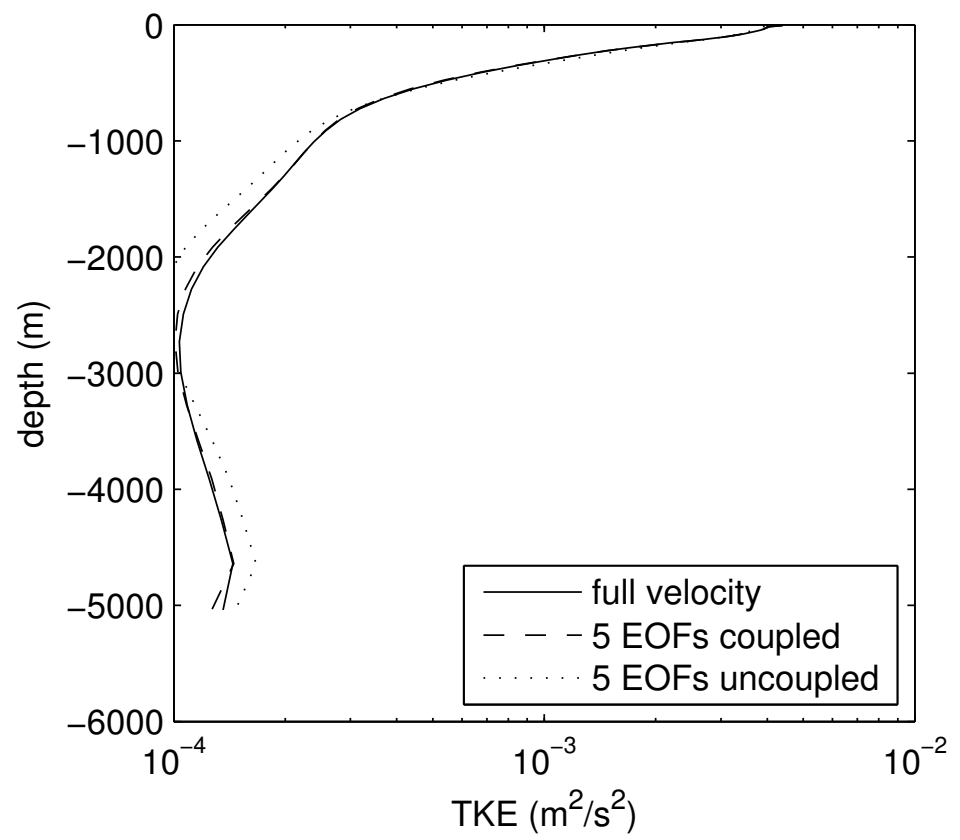

Figure 3-14: As Fig. 3-9, but using the first five EOFs. In contrast to the classic Rossby wave modes, the EOFs reconstruct the model $\overline{T K E}$ profile without accounting for mode coupling.

fields. For the region discussed above, near $35^{\circ} \mathrm{N}, 220^{\circ} \mathrm{E}$, the RMS amplitudes of the barotropic and first four baroclinic modes for the high-pass filtered velocity are in the ratio 0.9:1:0.4:0.3:0.2, very similar to those found for the unfiltered velocity field. The RMS mode amplitudes for low-pass filtered velocity fields (cutoff period 150 days) are identical to the unfiltered ratio. Decreasing the cutoff period to 90 days did not appreciably alter the results. Thus we find no evidence that the ratio of energy in the barotropic and baroclinic mode depends strongly on frequency.

We perform a similar analysis for wavelength, applying a high-pass filter with cutoff wavelength of $1000 \mathrm{~km}$. For the region near $35^{\circ} \mathrm{N}, 220^{\circ} \mathrm{E}$, the RMS amplitudes of the barotropic and first four baroclinic modes for the high-pass filtered velocity are in the ratio $0.9: 1: 0.4: 0.3: 0.2$. 


\subsection{Conclusions}

The first part of this chapter evaluates 10 years of output of the ECCO2 model in terms of the surface variability and vertical structure of currents. Overall, the ECCO2 model has an adequate representation surface variability and vertical structure of kinetic energy. Maps of SSH variance are qualitatively similar to observations. However, the wavenumber-frequency spectrum of SSH in quiet regions has too little westward propagating energy and too much eastward propagating energy relative to observations. The wavenumber-frequency spectrum is much closer to observations in high energy western woundary current regions. In terms of the vertical structure of kinetic energy, the model $\overline{T K E}$ matches current meter observations near the surface, but is too weak at depth. Splitting analysis into high and low energy regions, we see that the model does much better in high energy regions (western boundary current, equator, $\mathrm{ACC}$ ) than in most of the ocean. In low-energy regions, the vertical structure of $\overline{T K E}$ is not "too-baroclinic", as suggested by Penduff et al. [2006], but is too weak by a factor of 2 overall.

In the second part of this chapter, we explore the vertical structure of kinetic energy in the ECCO2 model in more detail. Decomposing the model velocity into the classic Rossby wave modes shows that the barotropic and first baroclinic modes typically dominate, with smaller contributions from higher modes. However, the barotropic and first baroclinic modes are strongly coupled-apparently they are not the correct dynamical modes to use for describing low-frequency currents in the model. The relative phase of the barotropic and first baroclinic modes, as well as EOF analysis, suggests that the correct dynamical modes should have a vertical structure similar to the first baroclinic mode, but shifted such that the amplitude approaches zero at the bottom.

To what extent can the results of the ECCO2 model vertical structure be applied

to the ocean? Current meter results have suggested that there is significant coupling between barotropic and baroclinic modes [Wunsch, 1997]. However, current meter records tend to be shorter than one year and have measurements at only a few vertical 
levels. These two issues make it difficult to estimate the degree of mode coupling from current meters. If the model $\overline{T K E}$ profile was "too baroclinic," as some have suggested, we would suspect that the result of the Section 3.4 were an artifact of the model. But we show model $\overline{T K E}$ is simply too weak overall, and the results may not be an artifact.

Any mode coupling has important consequences for the vertical structure of variability, and must be incorporated into the vertical structure of the four-dimensional model spectrum that is the goal of this thesis. The next chapter proposes a dynamical explanation for the apparent mode coupling. 


\section{Chapter 4}

\section{Dynamics of low-frequency variability}

The goal of this chapter is to explore the dynamics that influence the observed spectrum of ocean variability. It is too much to expect a full theory of the observed spectrum, and no single process could explain all the observations. But having some theoretical basis for the model spectrum is useful. In particular, a theory is sought to explain two key observations from previous chapters: the dominant phase speed of propagating SSH features and the associated vertical structure in the ECCO2 model. In return, the theory will suggest new questions for the observations.

Section 4.1 describes several existing theories for low-frequency oceanic variability. We begin with so-called "extended QGPV dispersion relations", which take into account the effect of mean flow and large-wavelength sloping topography (topographic wavelengths $L_{b}$ much larger than the wavelength of the motion $k^{-1}$ ) on the propagation of Rossby waves. The most promising of the extended theories is that of Killworth and Blundell [2005]. Second, we consider the implications of baroclinic instability. Finally we discuss the vertical structure and spectrum of variability forced by the atmosphere.

The main focus of this chapter is on developing the theory for the impact of rough topography on Rossby waves. Sections 4.2-4.5 considers the affect of small-wavelength topography on Rossby wave propagation. In these sections, we focus on the affect of 
topographic variations with wavelength $L_{b} \ll k^{-1}$. The results presented here extend earlier idealized studies to the case of realistic stratification and topography.

\subsection{Existing theories}

Chelton and Schlax [1996] observed what they described as large-wavelength planetary waves propagating faster than the classic linear free-wave theory predicted, prompting a large number of papers attempting to explain the observations. Initial efforts to explain the observations focused on extending the classic theory for linear Rossby waves to account for mean flow and gently sloping topography [Killworth et al., 1997; Killworth and Blundell, 2004, 2005] as well as steep topography [Tailleux and McWilliams, 2001] $]^{1}$.

More recently, the altimetric observations from the higher-resolution AVISO data product have been reinterpreted as nonlinear coherent vortices ${ }^{2}$ [Chelton et al., 2007, 2011]. However, they note that the smaller-wavelength coherent vortices propagate slightly slower than larger-wavelength Rossby waves observed in the earlier study. Nonlinearity plays an important role in the propagation of coherent vortices [Early et al., 2011].

Below, we discuss the relevance of extended linear wave theories, baroclinic instability, and surface forced motions for the observed low-frequency ocean variability. In particular, we discuss the ability of each theory to account for the observed phase speed and vertical structure of variability. These three topics may appear quite disparate, but all have been invoked to explain some aspect of the observed ocean variability. Each provides important context for discussing the spectrum of ocean variability.

\footnotetext{
${ }^{1}$ See $\S 1.1$ for a more complete list of references.

${ }^{2}$ Chelton et al. [2011] used the term "nonlinear eddy" to describe coherent mesoscale features for which rotational fluid speeds $U$ exceed their translation speed $c$, resulting in an advective nonlinearity ratio $U / c>1$. We use the term "nonlinear coherent vortex" as it is more descriptive and because the term "eddy" is overloaded in oceanography.
} 


\subsubsection{Extended linear Rossby wave theories}

Killworth et al. [1997] showed that a background zonal mean flow, which alters the mean potential vorticity gradient, modifies the speed of long Rossby waves. They found that the baroclinic part of the mean flow increased westward phase speeds over most of the ocean. This theory was extended to include arbitrary mean flow and large wavelength sloping topography [Killworth and Blundell, 2004, 2005].

An alternative explanation for the anomalously high phase speeds observed was provided by Tailleux and McWilliams [2001]. They argue that random, steep topography couples the barotropic and first baroclinic modes, increasing phase speeds. They call this effect "bottom pressure decoupling" since the bottom pressure anomalies are decoupled from the upper ocean dynamics. More detailed theoretical Rossby wave studies have shown that rough topography does produce bottom pressure decoupling and increase Rossby wave phase speeds [Samelson, 1992; Reznik and Tsybaneva, 1999; Bobrovich and Reznik, 1999].

Aoki et al. [2009] compared the phase speeds observed in an ocean GCM with phase speeds predicted with mean flow and bottom pressure decoupling effects. They showed that the two theories together predict long wave phase speeds in excess of the model phase speeds. When finite wavelengths were used, the theory with mean flow and bottom pressure decoupling was in good agreement with the phase speeds from the model. Aoki et al. [2009] also compared the relative impact of the mean flow and bottom pressure decoupling theories on the predicted phase speed. In most of the ocean, the bottom pressure decoupling theory was shown to have a larger impact.

Both the mean flow and bottom pressure decoupling effects alter the vertical structure of planetary waves [Aoki et al., 2009]. The vertical structure of the first baroclinic mode with mean flow is somewhat surface intensified relative to the classic theory and retains the mid-depth zero-crossing. In the bottom pressure decoupling theory, the vertical structure goes to zero at the ocean bottom by construction, and loses the zero crossing of the classic theory first baroclinic mode.

Supposing that low frequency oceanic variability is dominated by linear planetary 
waves, the mean flow and bottom pressure decoupling (or, more generally, rough topography) theories together appear to explain the observed phase speed and vertical structure. We explore the effect of rough topography in detail later in this chapter.

\subsubsection{Baroclinic instability}

Claims that the mesoscale altimetric signal largely reflects nonlinear motions are based on the nondispersive nature of the features and a nonlinearity parameter: the ratio of the particle speed within a coherent vortex to the vortex translation speed [Chelton et al., 2007]. The nonlinearity parameter was found to be greater than one (suggesting nonlinearity) in most regions poleward of $20^{\circ}$, and was especially high in the western boundary current extension regions. Early et al. [2011] added further evidence for the hypothesis that the mesoscale altimeter signal is dominated by nonlinear features. They ran a shallow-water QG numerical model with and without nonlinearity, and seeded the model with random mesoscale features. With nonlinearity, the features remained coherent for a longer time than without nonlinearity. Also, zonal wavenumber-frequency spectra for the nonlinear simulation were similar to observations, while the linear simulation failed to reproduce the nondispersive structure observed.

Much of the ocean is baroclinically unstable, with wavelengths of maximum instability typically near the deformation radius [Killworth and Blundell, 2007; Tulloch et al., 2011]. Baroclinic instability can provide a source for kinetic energy, which then cascades larger and smaller wavelength in a turbulent energy cascade. The turbulent cascade can be halted by a combination of Rossby waves [Rhines, 1975], stratification [Smith and Vallis, 2001], or dissipative processes [Arbic and Flierl, 2004b; Thompson and Young, 2006], though the relative importance of these processes remains unclear.

LaCasce and Pedlosky [2004] showed that the large, low-frequency baroclinic Rossby basin modes are subject to instability. North of $25^{\circ} \mathrm{N}$, this instability acts fast enough to break the wave down into small wavelength eddies before it crosses the basin. South of $25^{\circ} \mathrm{N}$, the wave is able to cross the basin intact. They claimed that this instability was responsible for the anomalous phase speeds observed by altimetry. 
Tulloch et al. [2009] have claimed that the altimetric observations can be explained as the result of baroclinic instability and geostrophic turbulence. Equatorward of $30^{\circ}$, they find the wavelength for which the linear theory with mean flow produces the observed phase speed. Poleward of $30^{\circ}$ they claimed that the linear theory did not give a good fit to the observed phase speed for any wavelength. These observations were interpreted in terms of a transition from wavelike regime at low latitude, where eddy periods can transfer energy to linear Rossby waves and halt the inverse energy cascade, to a turbulent regime at high latitude where Rossby wave periods are too low for eddy energy to be converted to Rossby waves. Such a description is consistent with the conclusions of LaCasce and Pedlosky [2004], though the exact relation between the two theories is not clear. In Ch. 2, we saw no evidence of a dramatic shift in the spectrum at $30^{\circ}$ suggestive of a transition from wavelike to turbulent regime (Fig. 2-16).

While the studies discussed above do provide convincing evidence for the role of nonlinearity in the altimetric signal, they focus on the mesoscale [Chelton et al., 2007; Early et al., 2011] or on a single wavelength chosen to match the observed phase speed [Tulloch et al., 2009]. However, these studies ignored the broadband nature of the altimetric observations; signals exist at all wavelengthss up to the size of the basin and periods up to the length of the record. Other generation mechanisms or an inverse turbulent cascade (moving energy to larger wavelengths) are required to explain the full range of variability.

\subsubsection{Surface forced motion}

Geostrophic motions can be decomposed into interior modes and SQG solutions, as noted in the Ch. 1 [see Lapeyre and Klein, 2006, for details]. The classic interior modes discussed by Gill [1982] and used in Ch. 3 use flat, rigid upper and lower boundary conditions, neglecting the surface buoyancy anomaly, and result in a barotropic mode and set of baroclinic modes. In atmospheric SQG theory, on the other hand, it is assumed that the interior PV is zero and the flow is determined entirely by the surface buoyancy distribution [Held et al., 1995]. Lapeyre and Klein [2006] considered a 
modified SQG theory, in which the surface buoyancy anomaly was due only to advection of mean surface gradients, and also included an interior PV anomaly correlated with the surface buoyancy anomaly. They claimed that this modified SQG theory explained the vertical structure of ocean variability.

In practice, it is difficult to distinguish between SQG solutions and more traditional QG motion (i.e., interior modes) since the SQG solution for large wavelength disturbances and realistic stratification projects strongly onto the first baroclinic mode [LaCasce, 2012]. The main distinguishing feature is that the SQG solutions decay more rapidly below the surface than the first baroclinic mode. However, the combined effect of mean flow and rough topography alters the first baroclinic mode so that it decays more rapidly as well [Aoki et al., 2009].

Surface buoyancy anomalies are advected by the surface geostrophic flow [as considered by Lapeyre and Klein, 2006], forced by vertical advection through the Ekman velocity, and by the divergence of surface buoyancy fluxes. Separation into interior modes and SQG solutions is not the only avenue for modeling surface forcing of geostrophic motions. For example, Frankignoul and Müller [1979a] and Müller and Frankignoul [1981] estimated the response of a simple ocean model to Ekman forcing at the surface. Their results suggest that direct wind forcing is the dominant forcing mechanism for central ocean eddies. In the central Pacific, the wavenumber spectrum of vertical displacement predicted by their theory is in good agreement with observations, but in the more energetic western Pacific their theory cannot explain the strong large-wavelength eddy activity. This suggests that baroclinic instability of boundary currents may be more important in more energetic regions. A related study found that the ocean response to surface buoyancy forcing is negligible [Frankignoul and Müller, 1979b].

In another example, Killworth and Blundell [2007] compared two types of forcing: vertical advection driven by the Ekman velocity and motion driven by surface buoyancy flux. In the case of a flat-bottom ocean with no mean flow, the response to Ekman forcing is much stronger than the response to buoyancy forcing, consistent with the findings of Frankignoul and Müller [1979b]. 
Killworth and Blundell [2007] also calculated the vertical structure of surfaceforced solutions. They found both oscillatory and surface trapped solutions in response to Ekman forcing. Recently, Smith and Vanneste [2012] have developed a relatively straightforward method for computing a "surface-aware" basis which includes both the classic free interior modes and surface-forced solutions as limiting cases. In general, their basis includes a barotropic interior mode, oscillatory baroclinic interior modes, and a decaying exponential surface solution. While the resulting modes have not been compared to data or model output, their approach is promising.

\subsection{Small-wavelength topography}

The interaction of large-wavelength barotropic Rossby waves with small-wavelength "rough" topography was explored by Rhines and Bretherton [1973]. We begin with a heuristic description of the effect, which will be made more concrete in the following sections, and review of earlier related results.

A large wavelength displacement over corrugated topography initially induces flow up and down the small-wavelength slope, and the fluid moving through a changing depth alters its relative vorticity leading to a pattern of flow along ridge crests and valleys (Fig. 4-1a). Variation of the initial displacement in the $x$-direction will lead to convergence/divergence at the ridge crests/valleys. The convergence/divergence leads to flow downslope everywhere near a node of the large wavelength displacement, and the downslope flow stretches the water column inducing relative vorticity generation in a sense that opposes the initial displacement (Fig. 4-1b). This induced relative vorticity acts to dampen the flow near topography (Fig. 4-1c). Rough topography effectively reduces the amplitude of the flow below the thermocline making the mode "more barotropic", in the sense that it increases the vertically integrated flow.

The approach used by Bobrovich and Reznik [1999] to solve the rough topography problem for a stratified ocean took account of the multi-scale interaction inherent in the dynamics. They began with the linearized QGPV equation written in terms of pressure, and restricted attention to the case of constant buoyancy frequency and 
a)

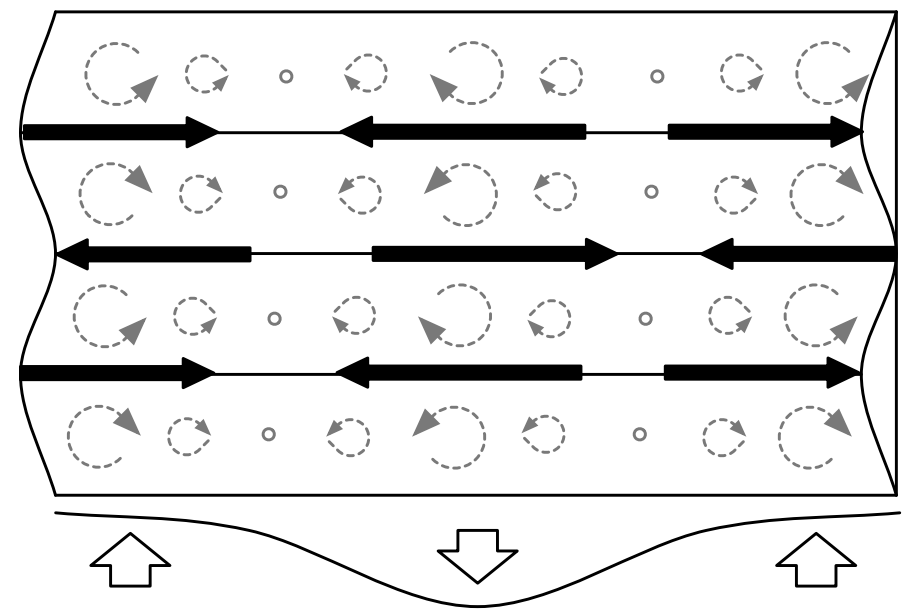

b)

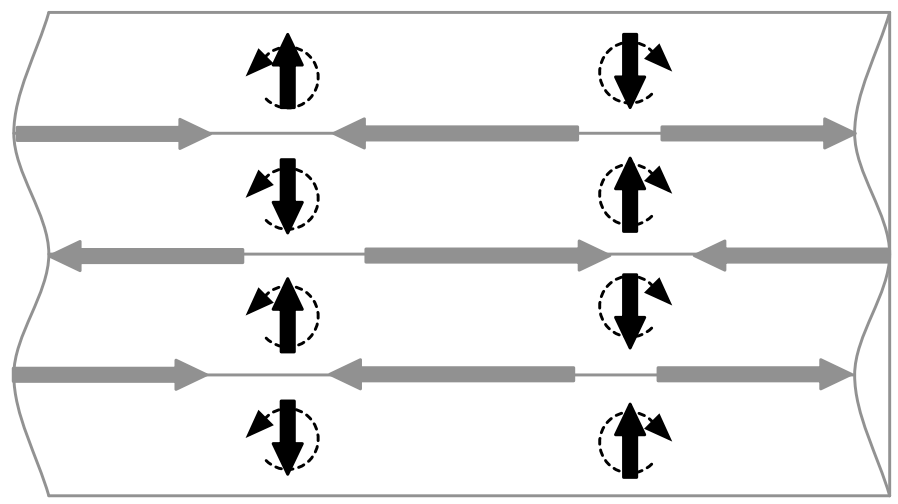

c)

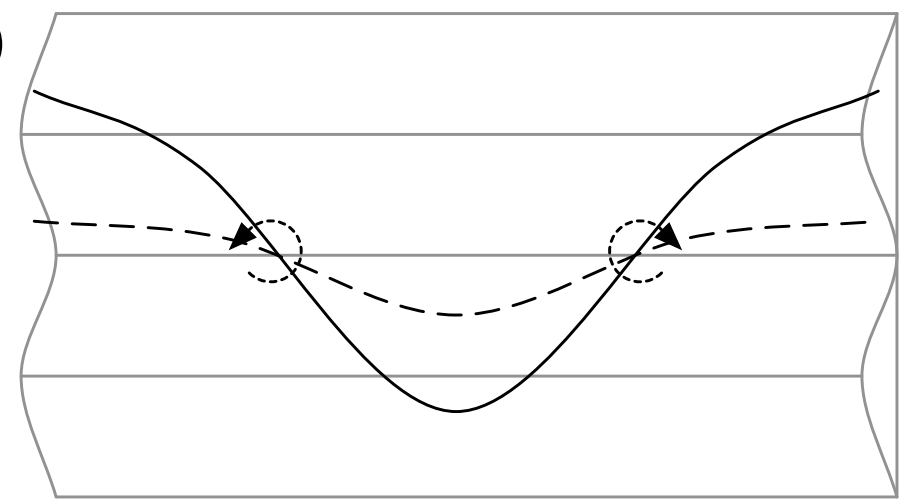

Figure 4-1: Large-wavelength Rossby waves can interact with rough topography. (a) A wave-like displacement over corrugated topography leads to a change in relative vorticity, and flow along the ridges and troughs. (b) The resulting convergence/divergence at crests/troughs drives flow downslope at one node of the initial displacement, and upslope at the other. The up/downslope flow generates relative vorticity at nodes of the initial displacement. (c) The sense of the relative vorticity tends to damp the initial displacement. Wavy lines represent large-wavelength wave displacement; solid arrows represent induced flow; dashed arrows represent induced relative vorticity. Adapted from Rhines and Bretherton [1973] Fig. 2. 
(nearly arbitrary) one dimensional topography $h=h(y)$. They sought solutions harmonic in $x$ and $t$, solving for the $y$ and $z$ dependence. While the full solution varies on the small topographic wavelength, their two-scale analysis assumes that the large wavelength variations are stronger than the small wavelength variations. This final assumption is not satisfied in the most interesting case of baroclinic modes over strong topography. Alternative boundary layer solutions do not make assumptions about the relative strength of large and small wavelength pressure variations, but instead apply significant restrictions to the topography. Neither approach is well suited to predicting phase speeds in the real ocean.

Despite their limitations, the results of Bobrovich and Reznik [1999] do provide some useful insight. First, in the limit of large amplitude topography (where their analysis is not formally valid) with constant stratification $N$, the baroclinic mode frequencies approach

$$
\omega_{n}=-\frac{\beta k}{k^{2}+l^{2}+\left(\pi f_{0} / N H\right)^{2}(n-1 / 2)^{2}}, \quad n=1,2,3, \ldots
$$

In the long-wave limit, they predict a frequency (and hence phase speed) four times that found in the classic, flat-bottom theory of Rossby waves. Observed phase speeds are only about twice the classic theory prediction [e.g., Chelton and Schlax, 1996], suggesting a failure of their mathematical approach in this limit. Second, they showed that the transition from the flat-bottom limit to the rough-bottom limit occurs smoothly, and the phase speed increase plateaued quickly as the relative height $\delta \sim \Delta H / H$ of the topography increased (their Fig. 2). That is, there is no frequency increase in the long-wave limit for $\delta=0$, the frequency is increased by a factor of four over the classic theory for $\delta \sim .01$, and there is no further increase in frequency as $\delta$ increases.

Tailleux and McWilliams [2001] took a different mathematical approach to account for the effect of rough topography. They conjectured that barotropic and baroclinic modes over random topography should be coupled in such a way as to diminish the bottom velocity. This mode coupling leads to an increase in baroclinic 
mode phase speed by a factor

$$
\frac{c_{\text {topo }}}{c_{\text {flat }}}=1+\frac{F_{1}^{2}(-H)}{\frac{1}{H} \int_{-H}^{0} F_{1}^{2}(z) \mathrm{d} z}>1
$$

where $c_{\text {topo }}$ is the phase speed over rough topography, $c_{\text {flat }}$ is the standard flat bottom phase speed, and $F_{1}(z)$ is the classic first baroclinic mode vertical structure (see (3.1)). One consequence of their mode coupling hypothesis is that it implies no flow at the level of the topography. This prediction is clearly contradicted by observations (Ch. 3).

While Bobrovich and Reznik [1999] and Tailleux and McWilliams [2001] took different mathematical approaches and investigated different aspects of the solution, they give equivalent results where they overlap. The results are directly comparable in the long wave limit with uniform stratification and strong topography. Both studies find a first vertical mode structure ${ }^{3} F_{1}(z) \sim \cos (\pi z / 2 H)$. As noted above, Bobrovich and Reznik [1999] calculate a maximum phase speed enhancement by a factor of four. For constant stratification, the ratio (4.2) is three, but this is only the leading term in a series expansion; results of a related Sturm-Liouville problem with vanishing pressure bottom boundary condition approach the factor of four found by Bobrovich and Reznik [1999].

These two studies take different but complementary approaches. The former considered the effect of topography amplitude with constant stratification, while the latter allowed for arbitrary stratification but assumed that topography had a large amplitude. We now extend the conclusions of these earlier studies to the case of realistic stratification and topography. A few questions will be of particular interest: Given realistic topography and stratification, which factor controls the phase speed enhancement? What is the implied vertical structure of kinetic energy? Do the enhanced phase speeds match observations?

\footnotetext{
${ }^{3}$ According to Tailleux and McWilliams [2001], the first mode vertical structure is given by their Eq. (62) plus an unspecified barotropic offset. We choose the offset such that the mode vanishes at $z=-H$.
} 
We begin with the linearized QGPV equation with a resting mean state:

$$
\frac{\partial}{\partial t}\left[\nabla^{2} p^{\prime}+\frac{\partial}{\partial z}\left(\frac{f_{0}^{2}}{N^{2}} \frac{\partial p^{\prime}}{\partial z}\right)\right]+\beta \frac{\partial p^{\prime}}{\partial x}=0
$$

where $p=\bar{p}(z)+p^{\prime}(x, y, z, t)$ is the pressure with perturbation $p^{\prime}, N(z)=-\left(g / \rho_{0}\right) \partial \bar{\rho} / \partial z$ is the buoyancy frequency, $f_{0}$ is the Coriolis parameter, and $\beta$ is the variation in the Coriolis parameter with latitude. The thermodynamic equation is

$$
\frac{\mathrm{D} b^{\prime}}{\mathrm{D} t}+w N^{2}=0
$$

where buoyancy $b=\bar{b}(z)+b^{\prime}(x, y, z, t)$ and $b^{\prime}=\left(1 / \rho_{0}\right) \partial p^{\prime} / \partial z$. Applying a rigid lid $(w=0)$ boundary condition at the upper surface gives

$$
\frac{\partial^{2} p^{\prime}}{\partial t \partial z}=0 \quad \text { at } \quad z=0
$$

At the bottom boundary, a vertical velocity is forced by flow up and down the slope: $w=u h_{x}+v h_{y}$. Using this in the thermodynamic equation and linearizing,

$$
\frac{f_{0}}{N^{2}} \frac{\partial^{2} p^{\prime}}{\partial t \partial z}=h_{x} p_{y}^{\prime}-h_{y} p_{x}^{\prime} \quad \text { at } \quad z=-H
$$

where $H$ is the mean depth and the bottom is at $z=-H+h$.

For simplicity, restrict attention to one dimensional topography $h=h(y)$ and look for solutions harmonic in $x$ and $t: p^{\prime}=P(y, z) \exp (i 2 \pi(k x-\omega t))$. Then

$$
\begin{gathered}
\frac{\partial^{2} P}{\partial y^{2}}+\frac{\partial}{\partial z}\left(\frac{f_{0}^{2}}{N^{2}} \frac{\partial P}{\partial z}\right)-\left(4 \pi^{2} k^{2}+\frac{k \beta}{\omega}\right) P=0 \\
\frac{\partial P}{\partial z}=0 \quad \text { at } \quad z=0 \\
\frac{\partial P}{\partial z}=\frac{N^{2} k}{f_{0} \omega} h_{y} P \quad \text { at } \quad z=-H
\end{gathered}
$$

Further analytical progress can only be made by assuming simple forms for topography $h$ or buoyancy frequency $N$, so at this point (4.4) is solved numerically with 
arbitrary topography and buoyancy frequency ${ }^{4}$. The case of uniform sloping topography, $h_{y}=$ constant, has been considered elsewhere; phase speeds may increase or decrease depending on the direction of the slope [Killworth and Blundell, 1999].

\subsection{Numerical solutions}

Equations (4.4) form a two-dimensional generalized eigenvalue problem for the modes $P(y, z)$ and eigenvalues $\omega$. The two missing boundary conditions are supplied by forcing the solution and its first derivative to be periodic in $y$. The equations are discretized on a rectangular grid with specified buoyancy frequency and topography. After discretization, (4.4) is written as a matrix equation

$$
\omega\left(\mathbf{A}+\mathbf{B}-4 \pi^{2} k^{2} \mathbf{I}\right) \mathbf{P}+\mathbf{D P}=0
$$

where $\mathbf{A}$ represents the horizontal derivative, $\mathbf{B}$ the stretched vertical derivative operator $\partial / \partial z\left(f_{0}^{2} / N^{2} \partial / \partial z\right)$, $\mathbf{D}$ the topography term in the bottom boundary condition, and $\mathbf{I}$ is the identity matrix. We solve the matrix eigenvalue problem for the modes $\mathbf{P}$ and eigenvalues $\omega$. See Appendix A for further details.

Buoyancy frequency profiles are based on $1^{\circ} \times 1^{\circ}$ gridded salinity and temperature profiles constructed for the Ocean Comprehensible Atlas (OCCA). OCCA is an ocean state estimate for the best-observed (at the time of publication) years 2004-2006 that employs observations (including surface altimetry, satellite SST, and Argo profiles) using a GCM and produces estimates of derived quantities (middepth dynamic topography; fluxes of heat and freshwater). For further details on OCCA, see Forget [2010]. Time-mean salinity and temperature profiles are averaged over $5^{\circ} \times 5^{\circ}$ before computing buoyancy frequency profiles at each point.

The topography is based on the ECCO2 model topography (see Ch. 3 for details on the model), which is in turn based on the Smith and Sandwell [1997] dataset. The shortest topographic wavelengths in the model are about $18 \mathrm{~km}$, the grid spacing. A

\footnotetext{
${ }^{4}$ The other obvious approach is separation of variables, but the finite amplitude bottom boundary condition is not separable for arbitrary topography. See Appendix A for further details.
} 


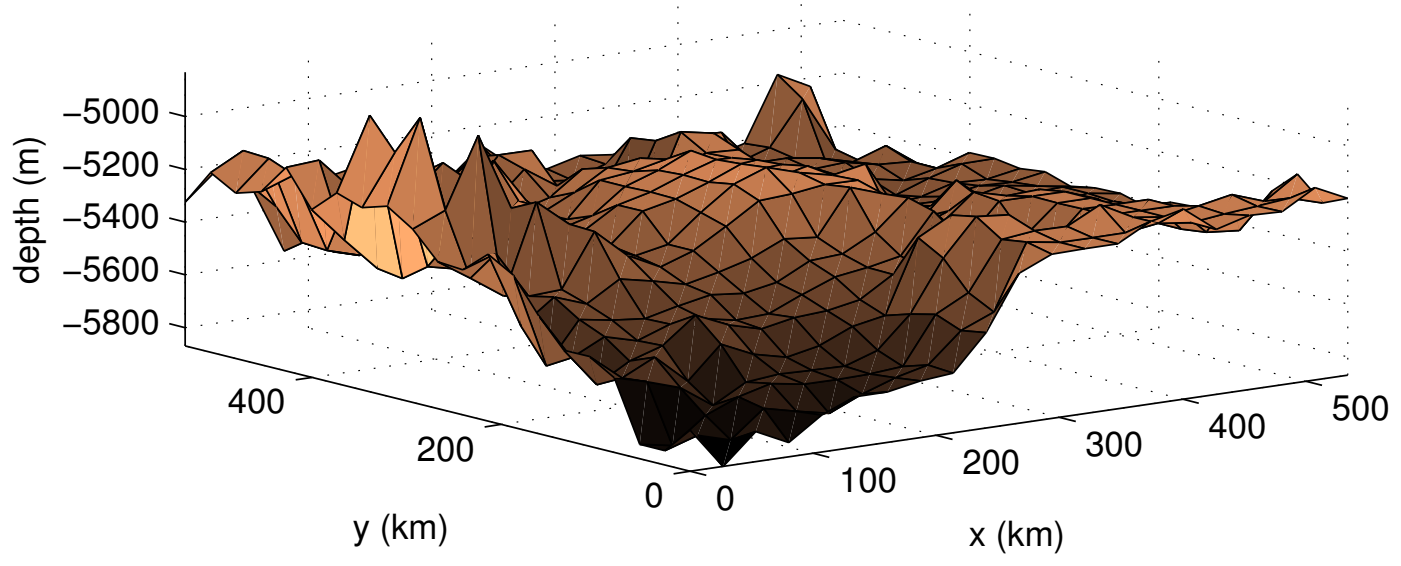

Figure 4-2: Typical area of bathymetric relief from the ECCO2 model.

typical area of bathymetric relief is shown in Fig. 4-2. The model topography is mapped onto a $1 / 4^{\circ} \times 1 / 4^{\circ}$ grid. Individual meridional topography sections are often dominated by a single seamount, or else miss all significant features, such that a single profile is not representative of a region. To make the results representative of a region as a whole, synthetic topography profiles are constructed based on the wavenumber spectrum at each point. Wavenumber spectra are computed along each meridional line in the $5^{\circ} \times 5^{\circ}$ box, averaged, truncated to remove wavenumbers longer than 300 $\mathrm{km}$, and used to generate a random realization of a topography profile having this spectrum. The random aspect of the ocean bathymetry leads to some variation in the predicted phase speed; based on multiple realizations of topography with the same spectrum, this variation is approximately $5 \%$ of the average phase speed.

\subsubsection{Mode structure}

Solutions to (4.4) cannot be ordered by the number of zero-crossings in the vertical, as in the flat bottom case (Fig. 4-3). The two-dimensional solutions $P_{n}^{m}(y, z)$ have both vertical and meridional modal structure, with $m=0,2,4, \ldots$ zero crossings in the horizontal and $n=0,1,2, \ldots$ in the vertical (Fig. 4-4). Superimposed on the basic vertical and meridional modal structure are variations in the meridional direction, which are required to satisfy the bottom boundary condition.

The barotropic mode and gravest baroclinic mode appear to be the most relevant 

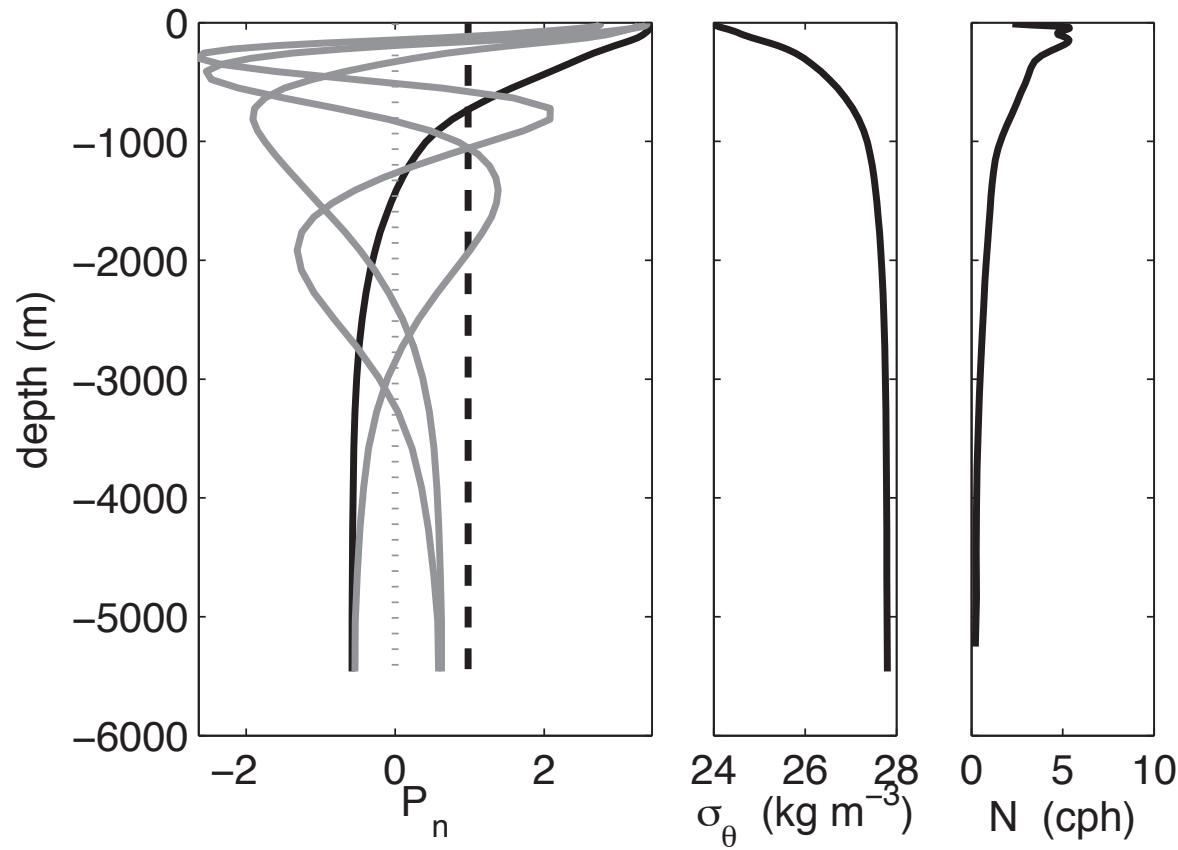

Figure 4-3: Flat bottom modes near $26^{\circ} \mathrm{N}, 150^{\circ} \mathrm{W}$ (left), potential density (middle), and buoyancy frequency (right). The barotropic (dashed), first baroclinic (solid), and higher baroclinic modes (grey) are shown.

for describing observations [Wunsch, 1997]. The pseudo-barotropic mode $P_{0}^{0}(y, z)$ is easily identified; it has the highest frequency and is nearly uniform in $y$ and $z$. Other pseudo-barotropic modes exist with some meridional structure.

Because no purely barotropic mode exists, the line between pseudo-barotropic and baroclinic mode is blurred. In practice, the gravest baroclinic mode $P_{1}^{0}(y, z)$ is identified using three criteria. 1) The mode is always positive at the surface: $P_{1}^{0}(y, 0)>0$. 2) The mode has its maximum value at the surface at every horizontal point: $P_{1}^{0}(y, 0)>P_{1}^{0}(y, z<0)$ for all $y$. 3) The meridional-average mode $P_{1}^{0}(z)=\int P_{1}^{0}(y, z) \mathrm{d} y$ has no internal maximum. For some wavenumbers and some topography profiles, two modes meet these three criteria, with similar meridionalaverage structure (i.e., always positive at the surface) but different variation around that average (Fig. 4-5, middle row). This duality appears as a near-degeneracy of the solutions; two eigenvectors have similar large scale structure, but differ in the details of the meridional structure. Physically, multiple ways exist to satisfy the bottom boundary condition for a given large scale mode structure. In this case, the mode 

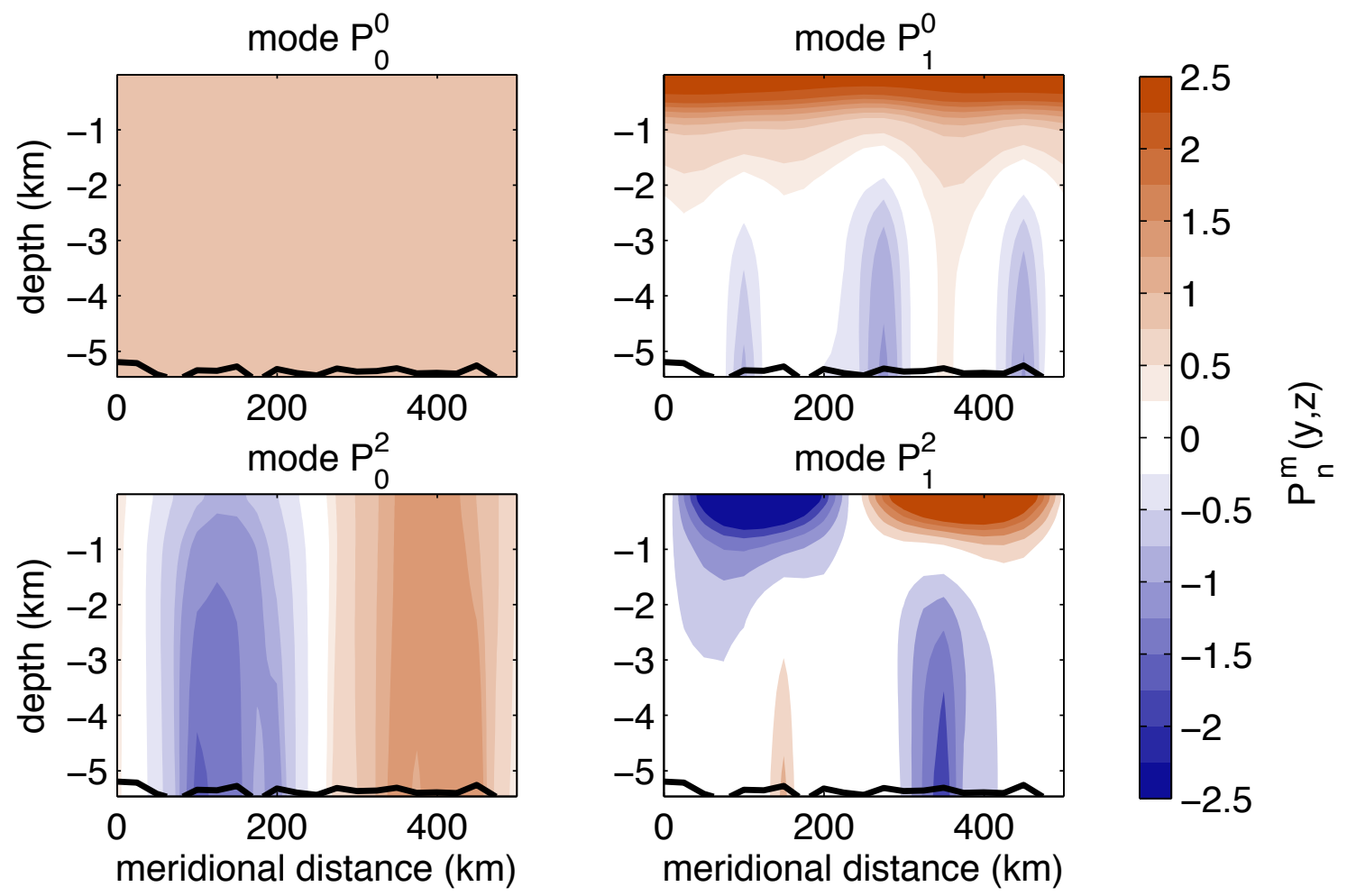

Figure 4-4: The solutions $P_{n}^{m}(y, z)$ of (4.4) are a set of vertical and meridional modes. Shown are the pseudo-barotropic mode (top left), gravest baroclinic mode with no meridional nodes (top right), pseudo-barotropic mode with two meridional nodes (bottom left), and the gravest baroclinic mode with two meridional nodes (bottom right) near $26^{\circ} \mathrm{N}, 150^{\circ} \mathrm{W}$ for $k=-4 \times 10^{-4} \mathrm{cpk}$. The black line is the topography profile. 
with the least variance at the bottom is chosen. As we describe below, the choice of one mode or the other can lead to discontinuities in the dispersion relation.

The solutions to (4.4) are ordered by the magnitude of their eigenvalues, and the behavior of a given solution (identified by its eigenvalue rank) evolves as wavenumber changes (Fig. 4-5). For example, the solution with the $5^{\text {th }}$ largest eigenvalue has a bottom-trapped structure at $k=-6.5 \times 10^{-3} \mathrm{cpk}$, but meets the baroclinic mode criteria set out above at $k=-2 \times 10^{-3} \mathrm{cpk}$ and $k=-6.5 \times 10^{-5} \mathrm{cpk}$. Meanwhile, the solution with the $9^{\text {th }}$ largest eigenvalue meets the baroclinic mode criteria at $k=-6.5 \times 10^{-3} \mathrm{cpk}$ and $k=-2 \times 10^{-3} \mathrm{cpk}$, but becomes a bottom-trapped mode at $k=-6.5 \times 10^{-5} \mathrm{cpk}$. When computing the dispersion relation for the gravest baroclinic mode, the dispersion curve will jump from one solution to the other at $k=-2 \times 10^{-3} \mathrm{cpk}$.

The observations considered so far, both of phase speed from altimetry and vertical structure in the ECCO2 model, reflect wavelengths larger than $\sim 100 \mathrm{~km}$. Accordingly, we will compare those observations with the "large-scale" (meridionalaveraged) vertical structure of the modes over topography. The averaged modes $P_{n}^{m}(z)=\int P_{n}^{m}(y, z) \mathrm{d} y$ form a set of pseudo-barotropic and baroclinic modes (Fig. 4$6)$.

The gravest baroclinic mean mode over topography maintains much of the shape of the flat bottom first baroclinic mode, but the amplitude is diminished at depth, consistent with observations of the vertical structure of currents in the ECCO2 model (Ch. 3). The diminished amplitude at depth is similar to a linear combination of the flat bottom barotropic and first baroclinic modes. Effectively, rough topography couples the barotropic and baroclinic modes in such a way as to reduce the bottom velocity.

If the barotropic and baroclinic modes are coupled by topography, as described by Tailleux and McWilliams [2001], one wonders how far from rough topography the barotropic and baroclinic modes will remain coupled. A complete answer likely requires numerical model simulations, because the answer depends on how the modes evolve as the wave propagates from rough to smooth topography. As a simplified 

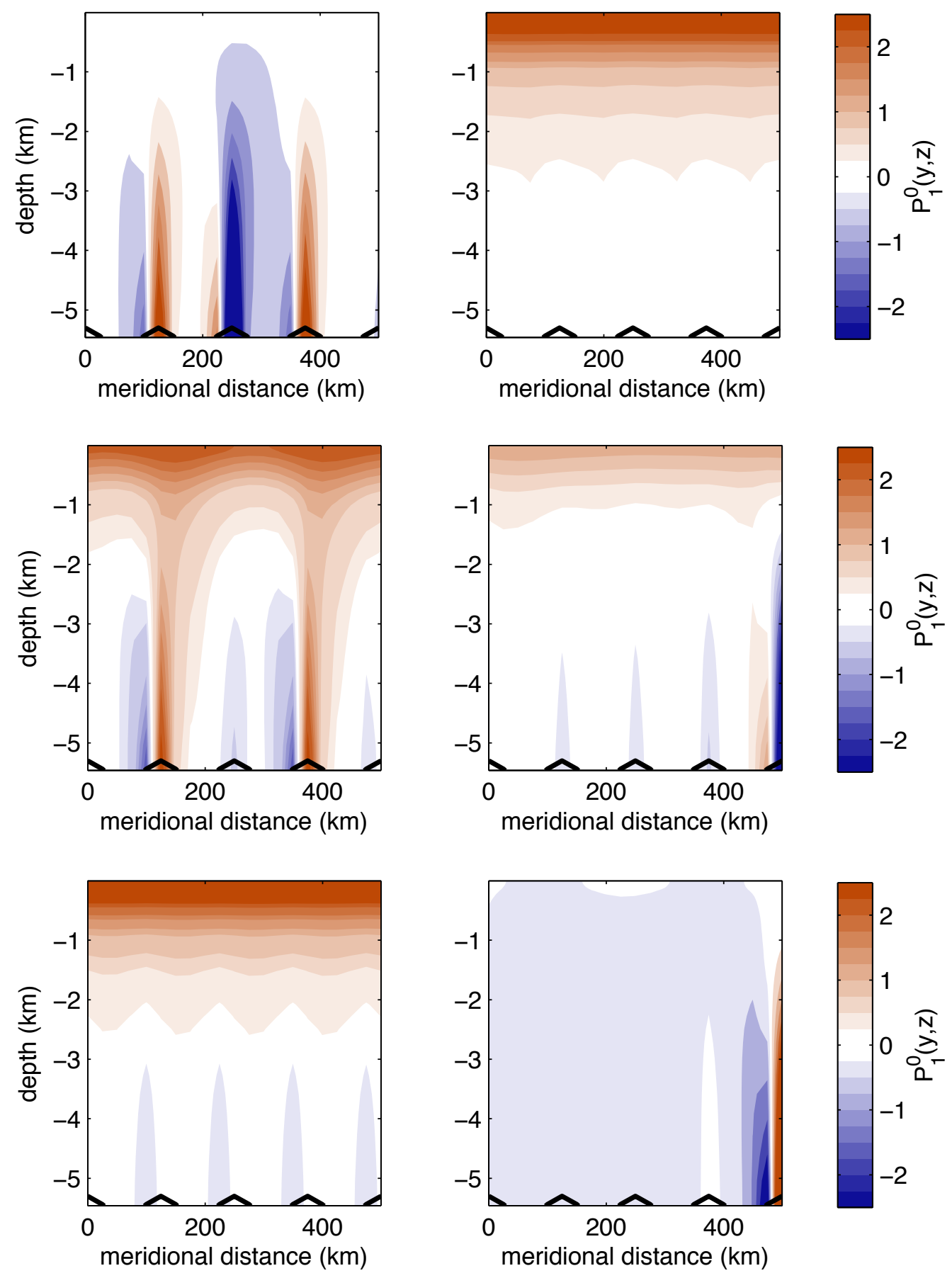

Figure 4-5: The behavior of a given solution to (4.4) (identified by its eigenvalue rank) evolves as wavenumber changes. At $k=-6.5 \times 10^{-3} \mathrm{cpk}$ (top) the $5^{\text {th }}$ solution (left) is bottom-trapped and the $9^{\text {th }}$ solution (right) meets the baroclinic mode criteria. At $k=-2 \times 10^{-3} \mathrm{cpk}$ (middle), both solutions meet the baroclinic mode criteria. By $k=-6.5 \times 10^{-5} \mathrm{cpk}$, the $5^{\text {th }}$ solution meets the baroclinic mode criteria, while the $9^{\text {th }}$ solution has become bottom trapped. The evolution of each solution occurs smoothly, but leads to a kink in the dispersion curve for the gravest baroclinic mode. 


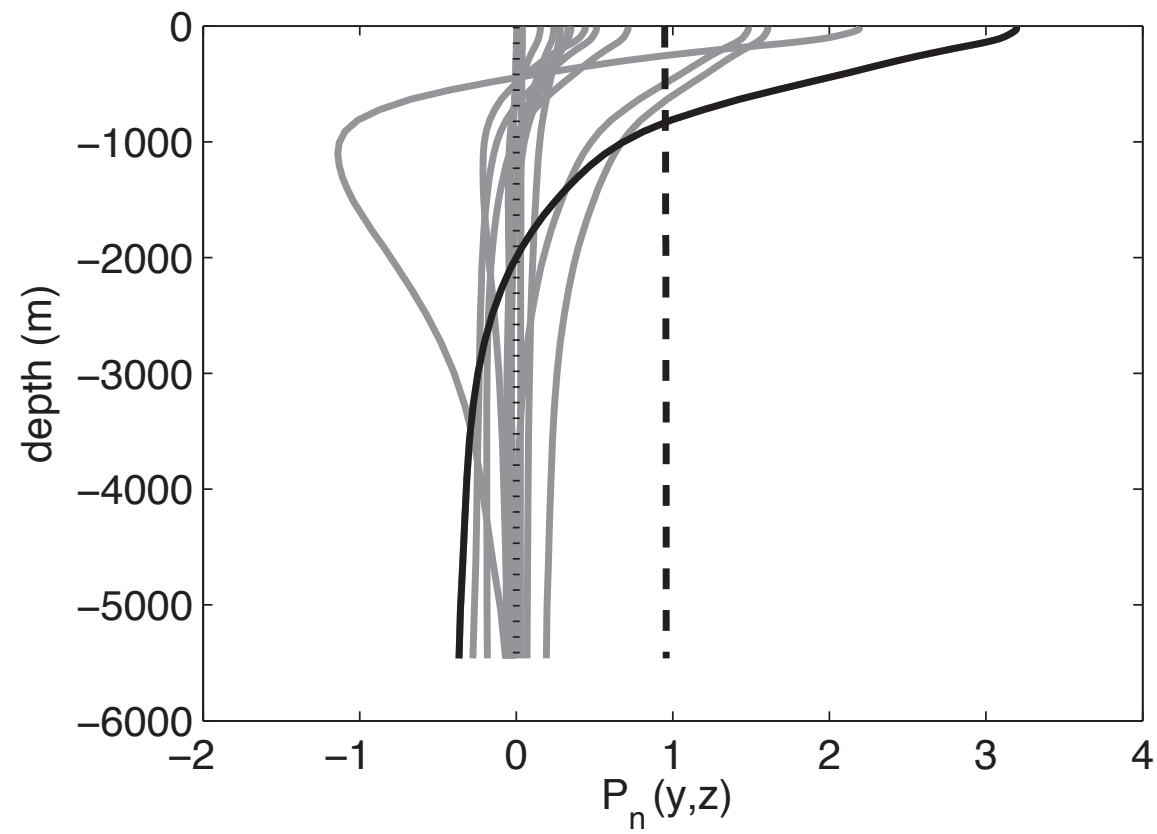

Figure 4-6: The first 16 mean modes $P_{n}^{m}(z)$ over topography near $26^{\circ} \mathrm{N}, 150^{\circ} \mathrm{W}$. Pseudo-barotropic mode (dashed), gravest baroclinic (solid), and higher baroclinic modes (grey) are shown. The baroclinic mode is diminished at depth relative to the flat bottom first baroclinic mode.

model, assume initially coupled barotropic and baroclinic modes propagate from rough to smooth topography, and maintain their frequency but change wavenumber in the process. The resulting waves will be out of phase after a distance

$$
x=\frac{\pi}{k_{0}-k_{1}}
$$

where $k_{0}$ and $k_{1}$ are the wavenumbers of the resulting barotropic and baroclinic modes, respectively. Consider a typical mid-latitude region with deformation radius $L_{d}=$ $50 \mathrm{~km}$, meridional wavenumber $l=0$, and frequency $\omega=7 \times 10^{-5} \mathrm{~s}^{-1}$ corresponding to the annual period. The barotropic mode with this frequency has wavenumber $k_{0}=-10^{-4} \mathrm{~m}^{-1}$, while two baroclinic modes have the same frequency: $k_{1}=(-9.6 \times$ $\left.10^{-5},-4.2 \times 10^{-6}\right) \mathrm{m}^{-1}$. The larger wavenumber suggests a decorrelation distance of $755 \mathrm{~km}$, while the smaller wavenumber suggests a decorrelation distance of 32 $\mathrm{km}$. However, results in $§ 4.5$ suggest that the ocean everywhere sufficiently rough to change the vertical structure. This fact, together with the ubiquitous mode coupling 
described in $\S 3.4$, suggests that decoupling of the barotropic and baroclinic modes is not relevant.

\subsubsection{Dispersion relation}

The dispersion relation for the gravest baroclinic mode reveals that the frequency is increased relative to the flat bottom mode, especially at low frequencies, indicating enhanced phase speed over topography (Fig. 4-7). While the magnitude of the phase speed enhancement depends on the details of the topography and stratification, Bobrovich and Reznik [1999] showed in their idealized cases that rough topography always leads to a phase speed increase and this remains true with realistic stratification.

The dispersion relation has a discontinuity near $k=-2 \times 10^{-3} \mathrm{cpk}$. Near this wavenumber, two solutions meet the gravest baroclinic mode criteria (Fig. 4-5). The discontinuity in the dispersion relation is the result of jumping from one baroclinic solution to another. The dispersion relations for the $5^{\text {th }}$ and $9^{\text {th }}$ solutions, discussed above, are shown. The gravest baroclinic mode is the $5^{\text {th }}$ solution for $|k|<2 \times$ $10^{-3} \mathrm{cpk}$, and switches to the $9^{\text {th }}$ solution for $|k|>2 \times 10^{-3}$ cpk. At small wavelengths, the $5^{\text {th }}$ solution has a bottom-trapped structure, while the $9^{\text {th }}$ solution is bottomtrapped at large wavelengths.

\subsection{Global results}

Equation (4.4) allows global prediction of the long Rossby wave phase speed with topography. Phase speeds are computed every $2^{\circ}$ of latitude and longitude where water depth is greater than $1000 \mathrm{~m}$ (Fig. 4-8). We use a wavenumber $k$ equal to $1 / 50^{\text {th }}$ of the local deformation radius $L_{d}$, resulting in a phase speed for the longwave limit.

Visually, the predicted phase speed with topography is not very different from the flat bottom phase speed, but the ratio reveals that the phase speeds with topography are enhanced by a factor of 1-2 in most regions (Fig. 4-9). Regions of stronger 


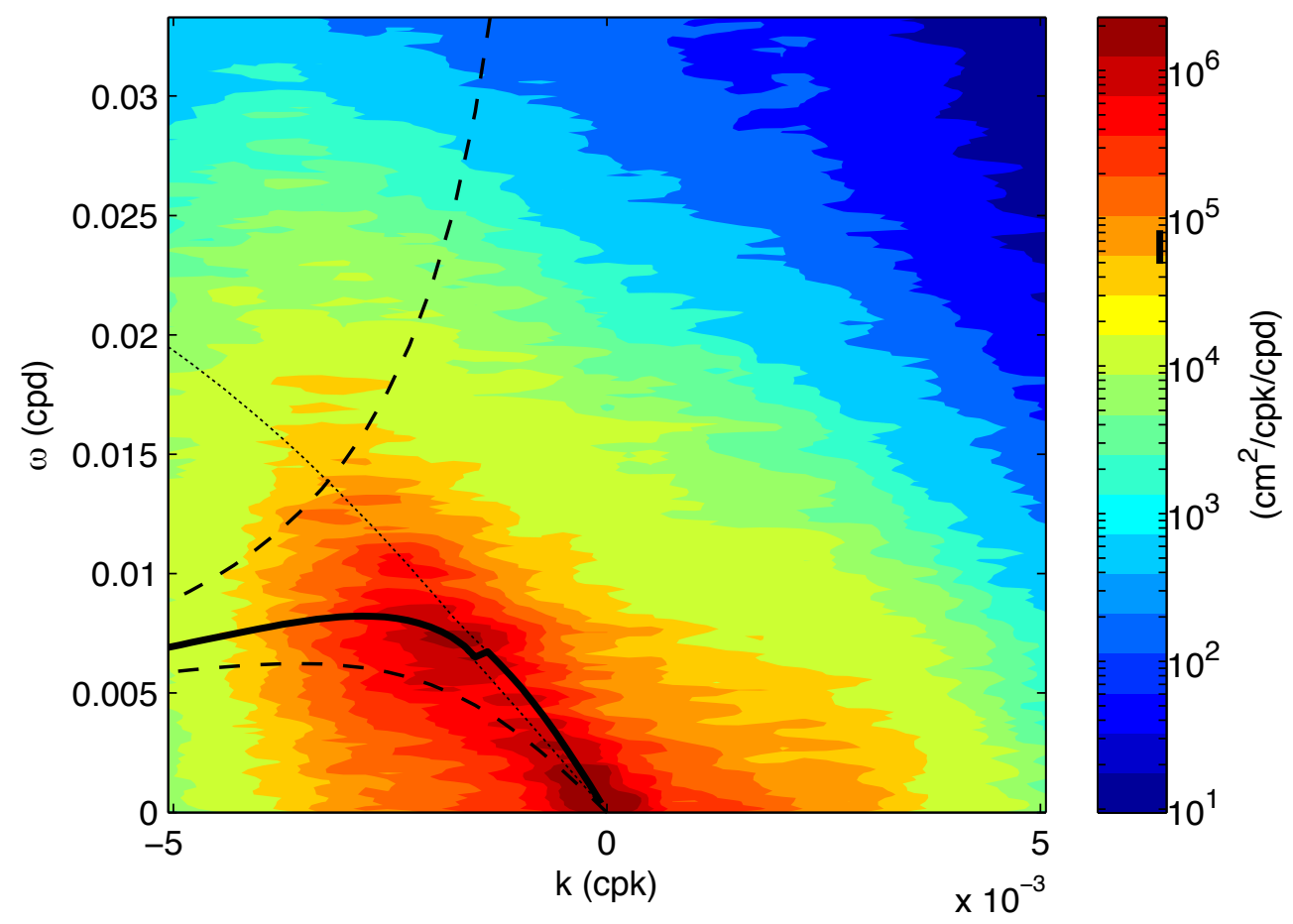

Figure 4-7: The dispersion relation for the gravest baroclinic mode with topography (solid) shows a higher phase speed at all wavelengths than the first baroclinic modes without topography (dashed) and is in close agreement with the dominant phase speed from the SSH spectrum (colored). Near the kink in the dispersion curve, two solutions exist with similar large-scale structure but different small-scale structure. The kink occurs when the criteria described in the text change from the $5^{\text {th }}$ solution to the $9^{\text {th }}$ (thin dotted lines). These solutions are shown in Fig. 4-5. Also shown is the barotropic mode without topography (dashed). The dispersion relations are calculated near $26^{\circ} \mathrm{N}, 150^{\circ} \mathrm{W}$ and the spectrum is averaged over $25^{\circ}-30^{\circ} \mathrm{N}, 130^{\circ}-$ $170^{\circ} \mathrm{W}$
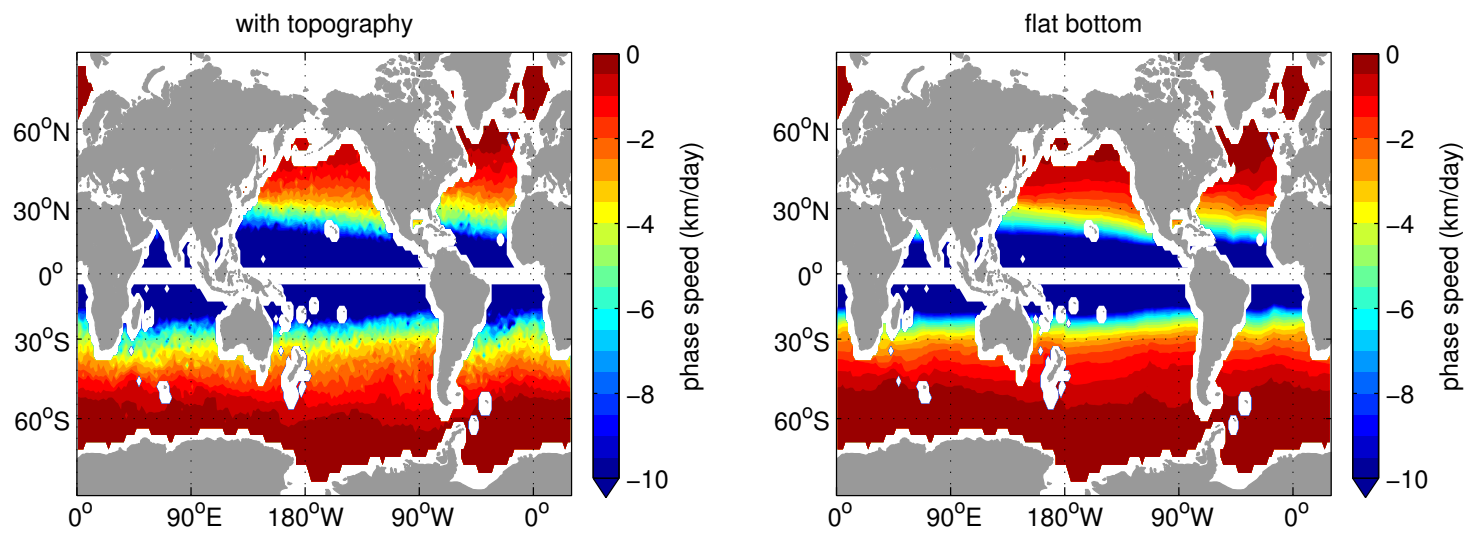

Figure 4-8: Predicted zonal phase speeds for long Rossby waves are everywhere higher with topography (left) than in a flat bottom ocean (right). Figure 4-9 shows the ratio. 


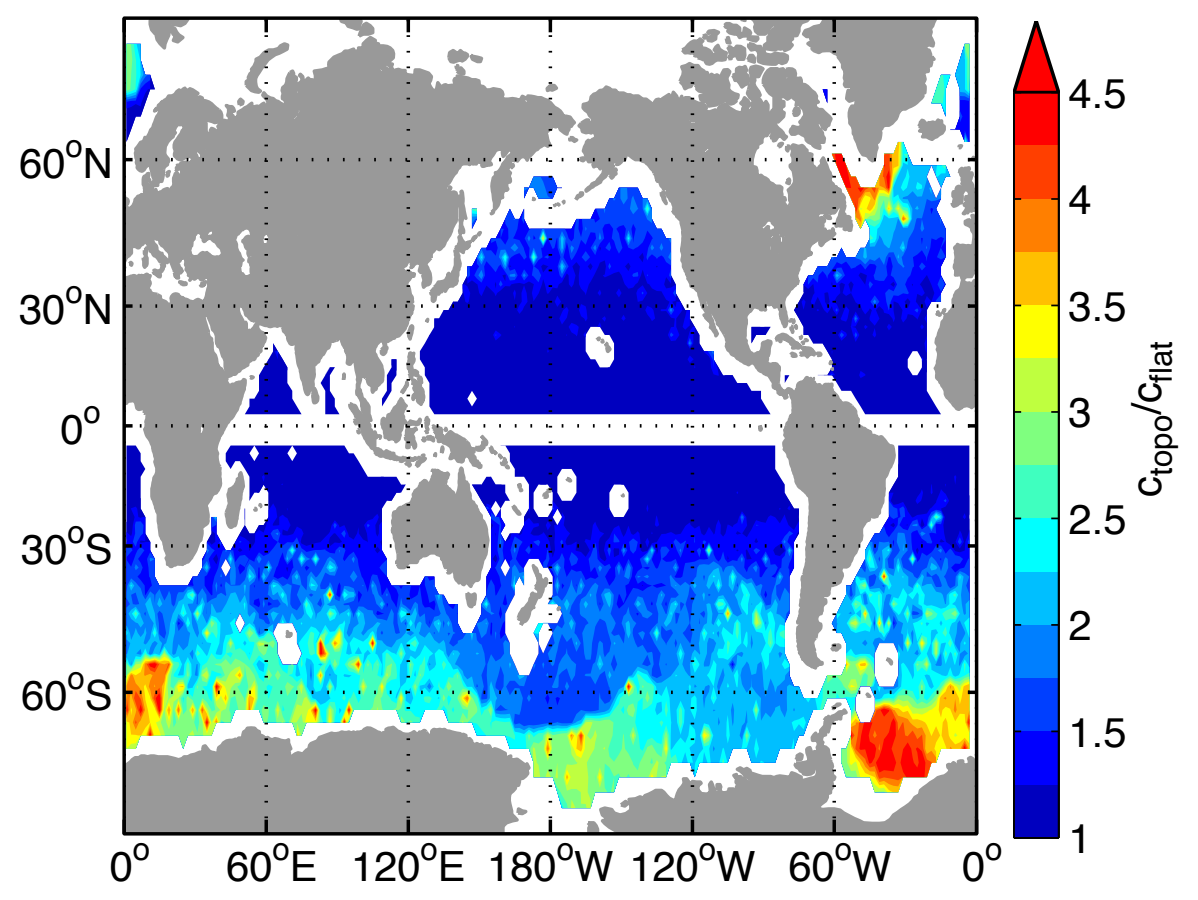

Figure 4-9: The ratio of the zonal phase speed for long Rossby waves with and without topography increases from $\sim 1$ near the equator to $\sim 2.5$ at $50^{\circ}$. The areas with phase speed enhancement greater than a factor of 3 are not relevant to observations, as described in the text.

phase speed enhancement $\left(c_{\text {topo }} / c_{\text {flat }}>3\right)$ are limited to high latitude areas of deep convection and low stratification, and are not relevant to observations for two reasons. First, the observational record is not yet long enough to show one wave crossing the basin poleward of $50^{\circ}$, even after doubling the phase speed [Chelton and Schlax, 1996]. Second, eastward mean currents associated with the ACC strongly affect wave propagation in the Weddell Sea, overwhelming the effect of rough topography.

In the zonal mean, Chelton and Schlax [1996] showed that observed long-wave phase speeds are enhanced relative the flat bottom prediction by a latitude-dependent factor, increasing from $\sim 1$ near the equator to $\sim 3$ at $40^{\circ}$. The small-wavelength coherent vortices discussed by Chelton et al. [2007] propagated slightly slower than largerwavelength Rossby waves. The impact of small wavelength topography accounts for much of this phase speed enhancement (Fig. 4-10). The magnitude depends on both the amplitude of the topography and on the stratification. The impact of these two factors will be considered independently below. 

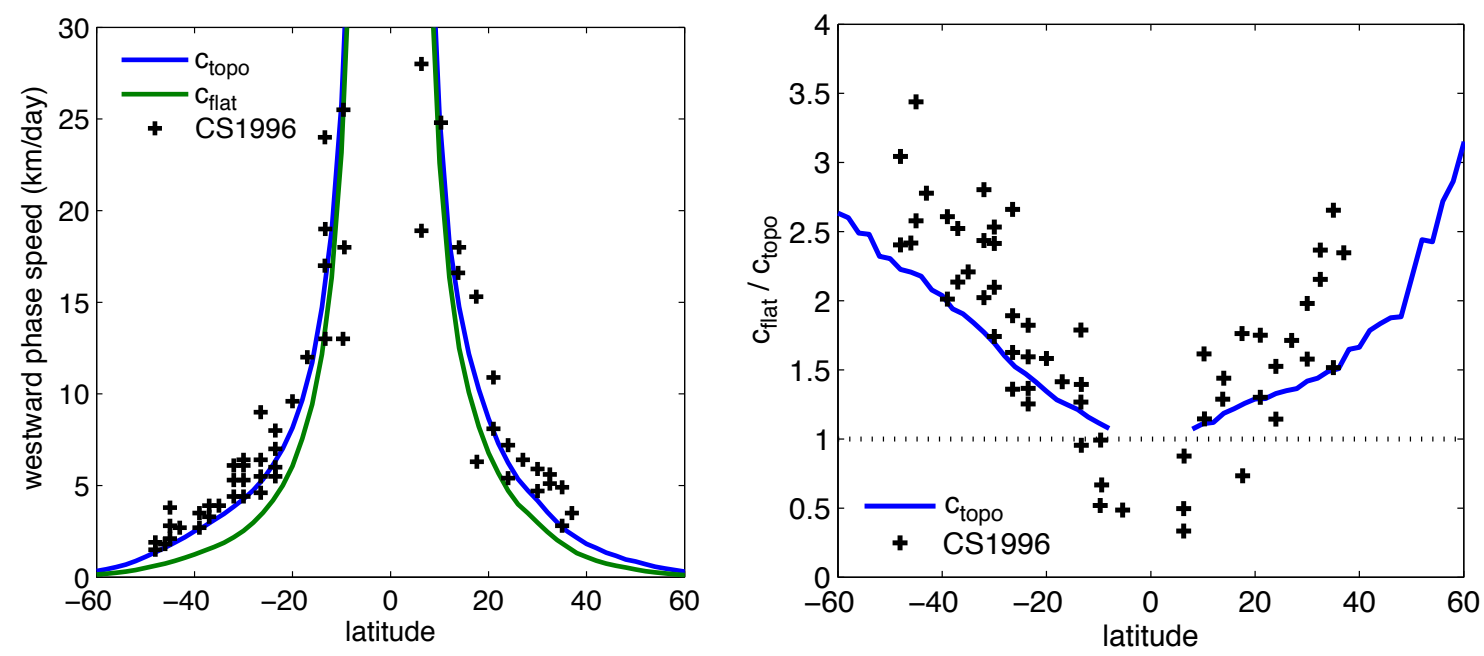

Figure 4-10: Zonal average zonal phase speeds (left) with topography (blue), in a flat bottom ocean (green), and the observations from Chelton and Schlax [1996] (crosses). The ratio of the predicted phase speeds with topography to the phase speeds with a flat bottom agree well with observations (right) at mid-latitudes, but observed phase speeds have a stronger latitude dependence than the prediction.

\subsection{Effect of stratification and topography ampli- tude}

Both Bobrovich and Reznik [1999] and Tailleux and McWilliams [2001] considered the effect of rough topography on Rossby waves in idealized limits. The former study used uniform stratification and showed that phase speed increased with topography amplitude, but that the effect saturated quickly (their Fig. 2). The later study assumed that the barotropic and baroclinic modes were coupled such that there was no flow at the ocean bottom, and gave an analytical expression for the phase speed increase as a function of stratification. However, the relative impacts of stratification and topography amplitude are not clear. Note that the phase speed increase, $c_{\text {topo }} / c_{\text {flat }}$, in Fig. 4-9 does not show any clear reflection of ocean bathymetry or RMS amplitude of the topography used in the calculation of the theoretical phase speed (Fig. 4-11). For example, the Mid-Atlantic Ridge and tropical western Pacific are areas of rough topography, but they do not show up clearly in the ratio $c_{\text {topo }} / c_{\text {flat }}$. We show below that the effect of globally variable stratification overwhelms the effect 

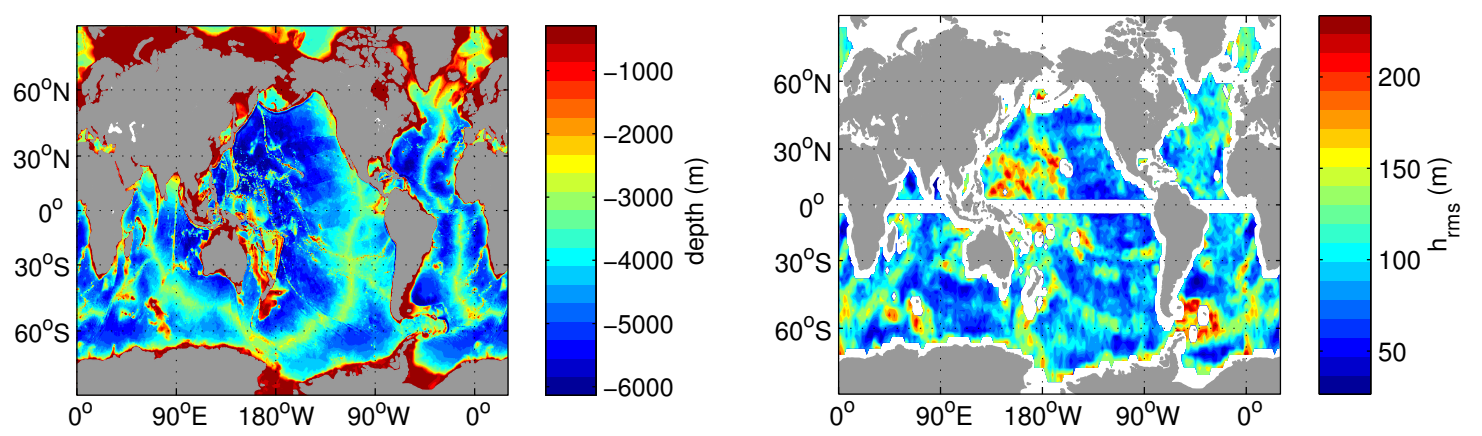

Figure 4-11: Map of bathymetry (left) and RMS topography height (right) used in the calculation of theoretical phase speed.

of topography amplitude, so that topography amplitude is not the dominant factor controlling the phase speed increase.

We plot the phase speed ratio $c_{\text {topo }} / c_{\text {flat }}$ as a function of topography amplitude for several different stratification profiles (Fig. 4-12). Phase speeds are calculated using a simple sinusoidal topography profile $h(y)=A \sin \left(8 \pi y / y_{\max }\right)$ with varying amplitude A. We use stratification from $\mathrm{OCCA}$ at $190^{\circ} \mathrm{E}$ and $10^{\circ} \mathrm{N}, 20^{\circ} \mathrm{N}, 30^{\circ} \mathrm{N}$, and $40^{\circ} \mathrm{N}$ to illustrate the impact of stratification. These latitudes represent different stratification profiles. The upper ocean stratification generally decreases with latitude (Fig. 4-13).

The phase speed increase depends on both stratification and topography amplitude. As amplitude increases, so does the phase speed ratio $c_{\text {topo }} / c_{\text {flat }}$. As predicted by Bobrovich and Reznik [1999], the effect of topography amplitude saturates, resulting in a maximum potential phase speed increase for a given stratification ${ }^{5}$. However, topography amplitudes are mostly in the 100-300 m range. In this range of amplitudes, the phase speed increase depends strongly on topography amplitude. If topography amplitude were the dominant factor determining the phase speed increase, we would expect to see areas of rough topography reflected in the phase speed map Fig. 4-9.

Geographical changes in stratification mitigate the impact of rough topography. Relatively weak stratification, as at $40^{\circ} \mathrm{N}$, results in higher maximum phase speed and more rapid approach to that maximum as topography amplitude increases. At $40^{\circ} \mathrm{N}$, topography amplitudes in the range 100-300 $\mathrm{m}$ result in phase speed ratios in the

\footnotetext{
${ }^{5}$ Continuing the plot to larger amplitude topography shows the saturation for the $10^{\circ} \mathrm{N}$ stratification.
} 


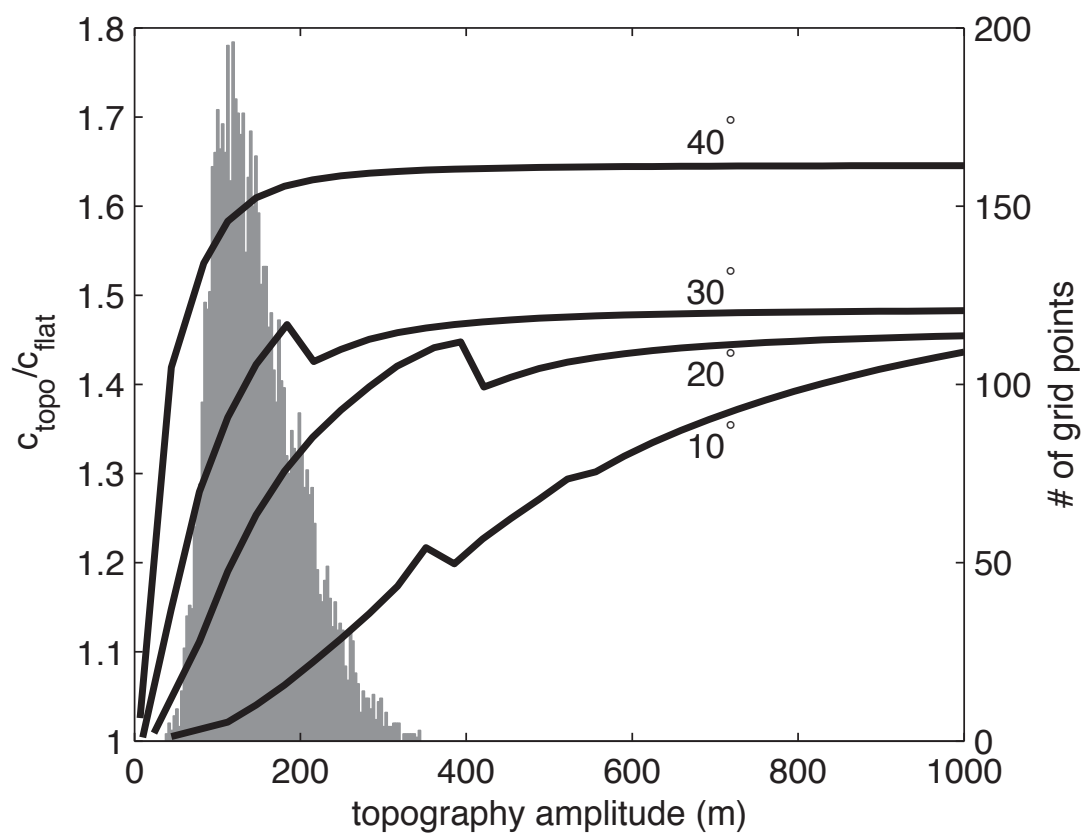

Figure 4-12: The phase speed increase with rough topography, $c_{\text {topo }} / c_{\text {flat }}$, depends on both the stratification and the topography amplitude (black lines, left axis). Lines show phase speed increase as a function of topography amplitude. Stratification is based on the OCCA climatology at $190^{\circ} \mathrm{E}$ and the latitude indicated. Also shown is a histogram of RMS topography height from Fig. 4-11 (grey bars, right axis).

range $1.55-1.65$. In contrast, at $10^{\circ} \mathrm{N}$, topography amplitudes in the range $100-300 \mathrm{~m}$ result in phase speed ratios in the range 1.03-1.15. In other words, even relatively low amplitude topography at $40^{\circ} \mathrm{N}$ results in a greater phase speed increase than does high amplitude topography at $10^{\circ} \mathrm{N}$. Thus, while both stratification and topography amplitude play a role in setting the phase speed increase, the ratio $c_{\text {topo }} / c_{\text {flat }}$ in Fig. 4-9 primarily follows the stratification rather than the bottom roughness.

\subsection{Conclusions}

The main contribution of this chapter is to extend the theory of linear Rossby waves over rough topography. The fact that small-wavelength rough topography could influence barotropic Rossby wave propagation was noted by Rhines and Bretherton [1973]. Bobrovich and Reznik [1999] and Tailleux and McWilliams [2001] considered the effect on baroclinic waves in idealized cases. Here, we compute the phase speed and 


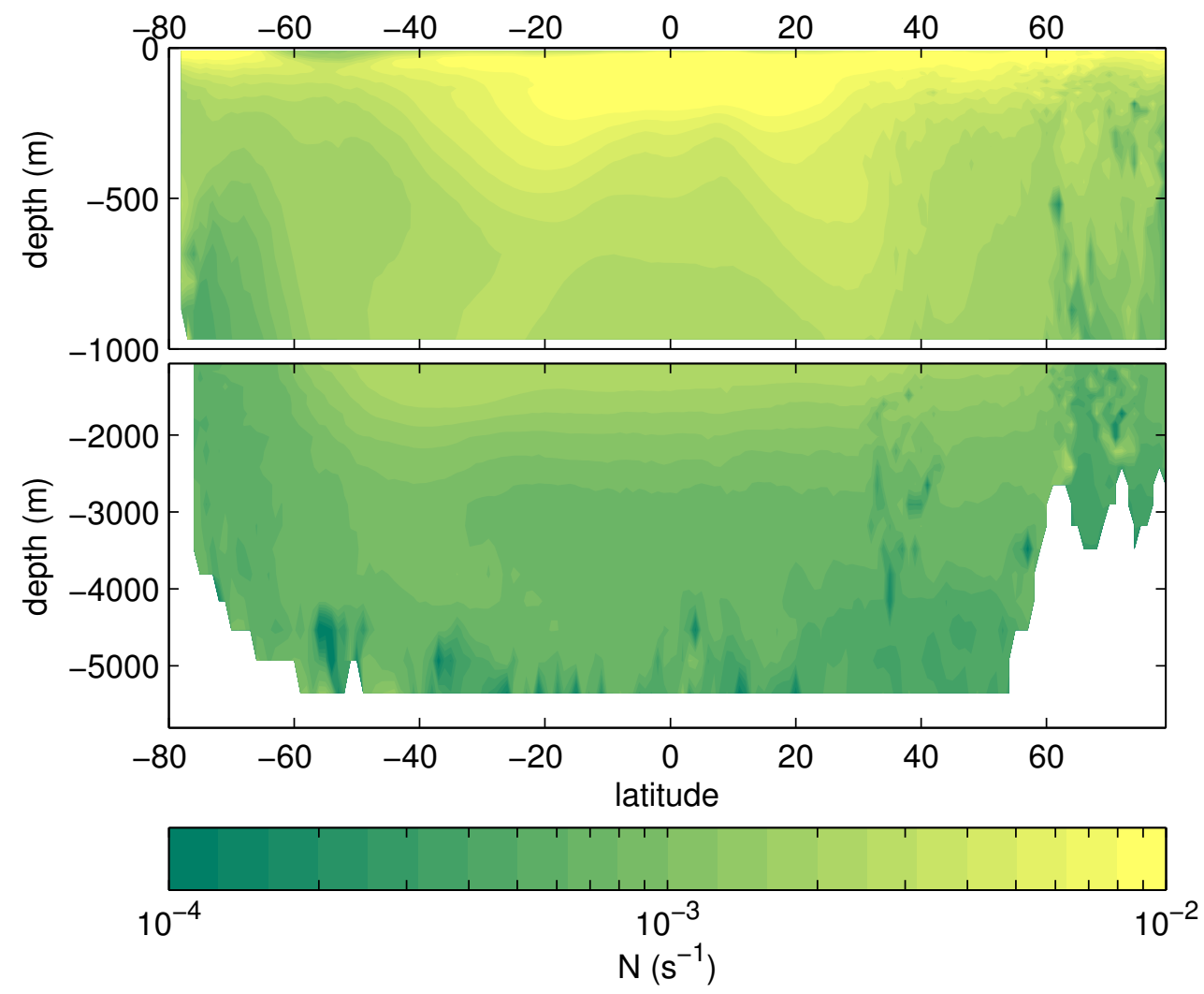

Figure 4-13: Zonal-mean buoyancy frequency from OCCA. Thermocline stratification is strongest at mid-latitudes, and weaker at the equator and high latitudes. This variation in stratification with latitude controls the degree of phase speed enhancement in the rough topography theory. 
vertical structure of Rossby waves over rough topography, using realistic topography and stratification. We are interested in the possibility that rough topography can explain two observations from earlier chapters: the "too-fast" propagation of features observed by satellite altimetry and the surface-enhanced vertical structure seen in the ECCO2 model.

We find that any rough topography increases Rossby wave phase speed relative to the classic theory. Maps of predicted phase speed show an increase by factor of 1-2 over most of the ocean (Fig. 4-9). Extreme increases in phase speed by a factor of 4 are confined to isolated areas of very low stratification and are unlikely to be observed.

The phase speed increase depends on both stratification and amplitude of topography (Fig. $4-12$ ). The ratio $c_{\text {topo }} / c_{\text {flat }}$ increases with topography amplitude, and even topography with an amplitude of order $10 \mathrm{~m}$ is sufficient to induce a phase speed increase. However, we find that stratification is more important than topography amplitude given the parameter range relevant to the ocean. Weaker stratification leads to greater phase speed ratio $c_{\text {topo }} / c_{\text {flat }}$, as predicted by Tailleux and McWilliams [2001]. We have not considered the dependence of phase speed on the characteristic wavelength of the topography in detail. However, the behavior in the limit of broad sloping topography was discussed by Killworth and Blundell [2004, 2005].

Rough topography also changes the vertical structure of waves. The resulting modes are enhanced near the surface and diminished at depth relative to the standard baroclinic modes. This is consistent with the "coupled mode" vertical structure seen in Ch. 3.

This study is limited to one-dimensional topography. Samelson [1992] studied the effect of rough topography in a two-layer QG model. Similar effects, including surface intensification and increased phase speed, were found for both one- and twodimensional topography. We restrict attention to one-dimensional topography so that we can compute analytical solutions, but this does not appear to be essential for the effect.

Overall, rough topography theory does predict phase speeds and vertical structures 
consistent with observations. However, there are many competing and overlapping theories for explaining the observed variability of geostrophic motion. We discussed several such theories in $\S 4.1$, but briefly review them here.

Both instability and forcing are likely important for generation of low frequency oceanic variability. We speculate that atmospheric forcing, through Ekman velocity as described by Müller and Frankignoul [1981], is the primary source for mid-ocean variability. Atmospheric forcing does not appear to be sufficient to explain the level of variability in the more energetic western boundary current and extension regions, where baroclinic instability of the mean flow is strongest [Smith, 2007; Tulloch et al., 2011]. While motions forced directly by the atmosphere appear to be broad band in space and time, baroclinic instability is strongest near or below the deformation wavelength [Killworth and Blundell, 2007; Tulloch et al., 2011] and turbulent energy cascades are likely responsible for transferring energy to both larger and smaller wavelengths.

Free, linear waves resulting from extended Rossby wave theories can easily explain the observed propagation speeds [Aoki et al., 2009]. However, none of the linear theories explain the nondispersive nature of mesoscale eddies, noted by Chelton et al. [2011], Wunsch [2010], and in Ch. 2. Nonlinearity appears to be important for explaining the coherence of mesoscale motions [Early et al., 2011].

Both atmospherically forced modes [Killworth and Blundell, 2007] and free interior modes over rough topography can explain the surface intensified vertical structure. Determining the relative contribution of each of these forces in setting the observed periods and wavelengths of variability, as well as propagation characteristics, remains an elusive goal. 


\section{Chapter 5}

\section{Model of the spectrum}

In this chapter, we propose and evaluate a comprehensive spectral description of lowfrequency ocean variability. We focus on the four-dimensional frequency-wavenumber spectrum of the streamfunction. Since the full spectrum of the streamfunction cannot be observed directly, it must be inferred from other observable spectra. This is an inverse problem: we wish to produce a spectrum of the streamfunction that is consistent with other observed spectra.

Zang [2000] proposed two models of the spectrum of the streamfunction, one isotropic and another attempting to account for the dominance of westward motions. Both spectra are separable functions of frequency, wavenumber, vertical mode, and location. However, the "ridge of westard variance" described in Ch. 2 cannot be accurately represented by a function separable in frequency and zonal wavenumber. Also, the shape of the zonal wavenumber-frequency spectrum (Fig. 2-16) changes with location, depending on the dominant phase speed. This change in shape of the spectrum implies a change in the dominant period and wavelength (Fig. 2-17), which cannot be represented in a model spectrum with constant shape. Finally, the proposed model partitions energy between the barotropic and low baroclinic modes for free, linear Rossby waves assuming that the modes are independent. As shown in Ch. 3, such a description underestimates the surface kinetic energy and overestimates the abyssal kinetic energy relative to observations. In this chapter, we propose an updated model of the streamfunction that attempts to address these issues. 
Müller and Frankignoul [1981] attempted to drive a spectral description of ocean variability from first principles. Their spectrum is based on direct atmospheric forcing of quasi-geostrophic motion. The resonant response of free, linear Rossby wave modes is a dominant feature of their derived spectrum. For baroclinic motions, they predict a sharp falloff in spectral power at frequencies above the high frequency limit of baroclinic Rossby waves. As noted in Ch. 2, there is significant energy at frequencies higher than the baroclinic frequency limit, even when the effects of rough topography are taken into account (Ch. 4). They also predict a two-dimensional wavenumberfrequency spectrum. This two-dimensional spectrum is dominated by resonance along the linear Rossby wave dispersion curves, also inconsistent with the observations in Ch. 2.

The full range of low-frequency ocean variability is unlikely to be determined by a single dynamical process (such as atmospheric forcing), and deriving an expression for the spectrum of variability resulting from multiple processes would be impractical. Our goal is not to determine the role of various dynamical processes in generating ocean variability, but to produce a comprehensive description of observed variability. Accordingly, our approach follows that of Zang [2000], proposing an empirical model of the spectrum rather than deriving a spectrum from first principles.

\subsection{Dynamic model for low-frequency motion}

We are interested in describing the variability of geostrophic motions. Zang [2000] took the linearized, quasi-geostrophic, $\beta$-plane equations [e.g., Gill, 1982] as a basic dynamical model and showed that the spectra of horizontal velocity, vertical velocity, vertical displacement, density, and pressure can all be derived from the spectrum of the streamfunction. In this section, we review this basic dynamical model introduced by Zang [2000] and the implied relations between observable spectra. 


\subsubsection{Governing equations}

As in Ch. 4, we begin with the linearized QGPV equation linearized about a resting mean state, (4.3). Assuming that the perturbation pressure is separable, we write

$$
p^{\prime}(x, y, z, t)=\sum_{n=0}^{\infty} P_{n}(x, y, t) F_{n}(z)
$$

where the vertical structure for each mode satisfies

$$
\frac{\mathrm{d}}{\mathrm{d} z}\left(\frac{f_{0}^{2}}{N^{2}(z)} \frac{\mathrm{d} F}{\mathrm{~d} z}\right)+\gamma^{2} F=0
$$

where $\gamma$ is a separation constant related to the deformation radius $L_{d}=1 / \gamma$. Given suitable top and bottom boundary conditions, (5.2) can be solved numerically for the verical modes $F_{n}(z)$. Flat bottom and rigid-lid boundary conditions are most common, and we discuss the rough-bottom case in Ch. 4. Vertical velocity and density are related to the vertical derivative of pressure, and so they will be described in terms of another vertical mode function

$$
G_{n}(z)=\frac{1}{N^{2}(z)} \frac{\mathrm{d} F_{n}(z)}{\mathrm{d} z}
$$

The horizontal structure for each mode is represented as a propagating wave

$$
P_{n}(x, y, t)=\int_{0}^{\infty} \int_{-\infty}^{\infty} \int_{-\infty}^{\infty} \tilde{p}(k, l, \omega, n) e^{i 2 \pi(k x+l y-\omega t)} \mathrm{d} k \mathrm{~d} l \mathrm{~d} \omega
$$

where $k, l$, and $\omega$ represent cyclic zonal wavenumber, meridional wavenumber, and frequency, respectively. $\tilde{p}(k, l, \omega, n)$ is the Fourier transform of the horizontal structure for mode $n$. The full solution for pressure in mode $n$ is

$$
p_{n}(x, y, z, t)=\int_{0}^{\infty} \int_{-\infty}^{\infty} \int_{-\infty}^{\infty} \tilde{p}(k, l, \omega, n) F_{n}(z) e^{i 2 \pi(k x+l y-\omega t)} \mathrm{d} k \mathrm{~d} l \mathrm{~d} \omega
$$


We define the Fourier transform of the streamfunction for mode $n$

$$
\tilde{\psi}(k, l, \omega, n)=\frac{\tilde{p}(k, l, \omega, n)}{\rho_{0} f_{0}}
$$

Assuming geostrophic dynamics, horizontal velocity, vertical velocity, vertical displacement, density, and pressure can all be derived from the streamfunction. For a generic variable $\chi_{n}(x, y, z, t)$, the $n^{\text {th }}$ mode is

$$
\chi_{n}(x, y, z, t)=\int_{0}^{\infty} \int_{-\infty}^{\infty} \int_{-\infty}^{\infty} \tilde{\chi}(k, l, \omega, z, n) \tilde{\psi}(k, l, \omega, n) e^{i 2 \pi(k x+l y-\omega t)} \mathrm{d} k \mathrm{~d} l \mathrm{~d} \omega
$$

Zang [2000] listed the characteristic functions $\tilde{\chi}(k, l, \omega, z, n)$, repeated here for convenience. For zonal velocity $u$, meridional velocity $v$, vertical velocity $w$, density $\rho$, vertical displacement $\zeta$, and temperature $\theta$,

$$
\begin{gathered}
\tilde{p}(k, l, \omega, z, n)=\rho_{0} f_{0} F_{n}(z) \\
\tilde{u}(k, l, \omega, z, n)=-i 2 \pi l F_{n}(z) \\
\tilde{v}(k, l, \omega, z, n)=i 2 \pi k F_{n}(z) \\
\tilde{w}(k, l, \omega, z, n)=i 2 \pi \omega f_{0} G_{n}(z) \\
\tilde{\rho}(k, l, \omega, z, n)=-\frac{\rho_{0} f_{0}}{g} N^{2}(z) G_{n}(z) \\
\tilde{\zeta}(k, l, \omega, z, n)=-f_{0} G_{n}(z) \\
\tilde{\theta}(k, l, \omega, z, n)=f_{0} \frac{\partial \theta_{0}}{\partial z} G_{n}(z)
\end{gathered}
$$

\subsubsection{Spectra of the model}

The three-dimensional frequency and wavenumber spectrum for the mode $n$ streamfunction is

$$
\Phi_{\psi}(k, l, \omega, n)=\langle| \tilde{\psi}\left(k, l, \omega,\left.n\right|^{2}\right\rangle
$$

where angle brackets represent an ensemble average. For other variables, the spectrum at depth $z$ and mode $n$ can be calculated from the spectrum of the streamfunction 
and the characteristic function:

$$
\Phi_{\chi}(k, l, \omega, z, n)=|\tilde{\chi}(k, l, \omega, z, n)|^{2} \Phi_{\psi}(k, l, \omega, n)
$$

More useful is the full spectrum at depth $z$, obtained by summing over all modes:

$$
\Phi_{\chi}(k, l, \omega, z)=\sum_{n=0}^{\infty}|\tilde{\chi}(k, l, \omega, z, n)|^{2} \Phi_{\psi}(k, l, \omega, n)
$$

Two-dimensional spectra can be derived by integrating the three-dimensional spectrum over one dimension ${ }^{1}$. For example, the two-dimensional wavenumber spectrum is

$$
\Phi_{\chi}(k, l, z)=\int_{0}^{\infty} \Phi_{\chi}(k, l, \omega) \mathrm{d} \omega
$$

Similarly, integrating over two dimensions gives the spectrum in the third dimension:

$$
\Phi_{\chi}(l, z)=\int_{0}^{\infty} \int_{-\infty}^{\infty} \Phi_{\chi}(k, l, \omega) \mathrm{d} k \mathrm{~d} \omega
$$

\subsection{Spatially varying $4 \mathrm{D}$ spectrum of streamfunc- tion}

As noted by Zang [2000], observed one-dimensional frequency spectra are approximately self-similar with depth, suggesting that the shape of the spectrum in horizontal wavenumber and frequency spectrum is independent of mode number. Accordingly, we propose a streamfunction spectrum of the form

$$
\Phi_{\psi}(k, l, \omega, n ; \phi, \lambda) \sim \Phi_{\psi}(k, l, \omega ; \phi, \lambda) E(n) I(\phi, \lambda)
$$

$\Phi_{\psi}(k, l, \omega ; \phi, \lambda)$ is the three-dimensional frequency-wavenumber spectrum, which changes slowly with latitude $\phi$ and longitude $\lambda . E(n)$ represents the contribution from each

\footnotetext{
${ }^{1}$ The notation for one, two, or three-dimensional spectra of several variables $q$ can be quite cumbersome. We use $\Phi_{\chi}(k, l, \omega, z)$ for the spectrum of $\chi$ at depth $z$, and the arguments $(k, l, \omega)$ denote the dimensionality of the spectrum.
} 
vertical mode $n . I(\phi, \lambda)$ sets the overall amplitude of the spectrum, and will be chosen to match the eddy kinetic energy estimated from altimetry.

\subsubsection{Surface streamfunction}

Observations of the three-dimensional spectrum of SSH reveal a few quasi-universal qualitative features, discussed in Ch. 2. The spectrum is dominated by a ridge of westward variance almost everywhere in the ocean. This ridge dominates at wavelengths larger than about $500 \mathrm{~km}$, and periods longer than two weeks at $10^{\circ}$ or longer than two months at $40^{\circ}$. At smaller wavelengths, the anisotropy diminishes, and the spectrum appears to approach an isotropic power law in wavenumber, though the resolution of the gridded altimetry product is insufficient to make a definitive statement at smaller wavelengths. At high frequency, the spectrum approaches an approximate power law.

Other qualitative aspects of the spectrum vary geographically. Most important, the amplitude of the spectrum varies over four orders of magnitude over the ocean (Fig. 2-4). The dominant phase speed increases equatorward, following the increase in baroclinic Rossby wave phase speed. Also, the wavenumber spectral slope in the 70-250 km band has a complicated, spatially variable pattern [Xu and Fu, 2012, Fig. 3].

Finally, note that the spectrum is broad-band in space and time. Energy exists at all periods and wavelengths, and there are no clear spectral peaks at the low frequencies considered here. The only exception is the annual period, which was suppressed in the SSH spectrum by removing the spatial mean SSH at each time step before computing the spectrum.

These features are captured by the function

$$
\begin{aligned}
\Phi_{\psi}(k, l, \omega ; \phi, \lambda)=\frac{1}{a} & {\left[\frac{d_{1}^{2 n} d_{2}^{2}}{\left(k^{2}+l^{2}+d_{1}^{2}\right)^{n}\left(\omega^{2}+d_{2}^{2}\right)}\right.} \\
& \left.\quad+\exp \left\{-\pi^{2}\left(k^{2} L_{x}^{2}+l^{2} L_{y}^{2}+T^{2}\left(k c_{x}+l c_{y}-\omega\right)^{2}\right)\right\}\right]
\end{aligned}
$$


where

$$
a=\int_{0}^{\infty} \int_{-\infty}^{\infty} \int_{-\infty}^{\infty}\left(|\tilde{u}|^{2}+|\tilde{v}|^{2}\right) \Phi_{\psi}(k, l, \omega ; \phi, \lambda) \mathrm{d} k \mathrm{~d} l \mathrm{~d} \omega
$$

is a normalization factor; and $n, d_{1}, d_{2}, L_{x}, L_{y}, T, c_{x}$, and $c_{y}$ are geographically variable parameters. Equation (5.21) has two parts. The first term is an power law, isotropic in $k$ and $l$, intended to describe the high frequency and wavenumber spectrum. The parameters $d_{1}$ and $d_{2}$ force the spectrum to be finite as $(k, l, \omega) \rightarrow 0$, while $n$ sets the high wavenumber spectral slope. The second term controls the low frequency and wavenumber shape of the spectrum, and in particular enforces the dominance of westward propagation. The parameters in this term set the dominant periods and wavenumbers of the spectrum, as well as the propagation speeds.

The full model spectrum is illustrated in Fig. 5-1 as a set of two-dimensional marginal spectra. Due to the difficulty of plotting positive and negative wavenumbers together on a logarithmic scale, we show the average of the positive and negative wavenumber half-spaces ${ }^{2}$. The spectrum is generally characterized as a low frequency/wavenumber plateau transitioning to a power law in frequency/wavenumber at smaller scales. An alternate three-dimensional illustration is shown in Fig. 5-2 for comparison with Fig. 2-7.

\footnotetext{
${ }^{2}$ Averaging over positive and negative wavenumber half-spaces reduces the prominence of the ridge of westward variance in the $k$ - $\omega$ spectrum slightly. The $l-\omega$ spectrum is symmetric about $l=0$ and is unchanged. Changes to the $k-l$ spectrum are slight.
} 

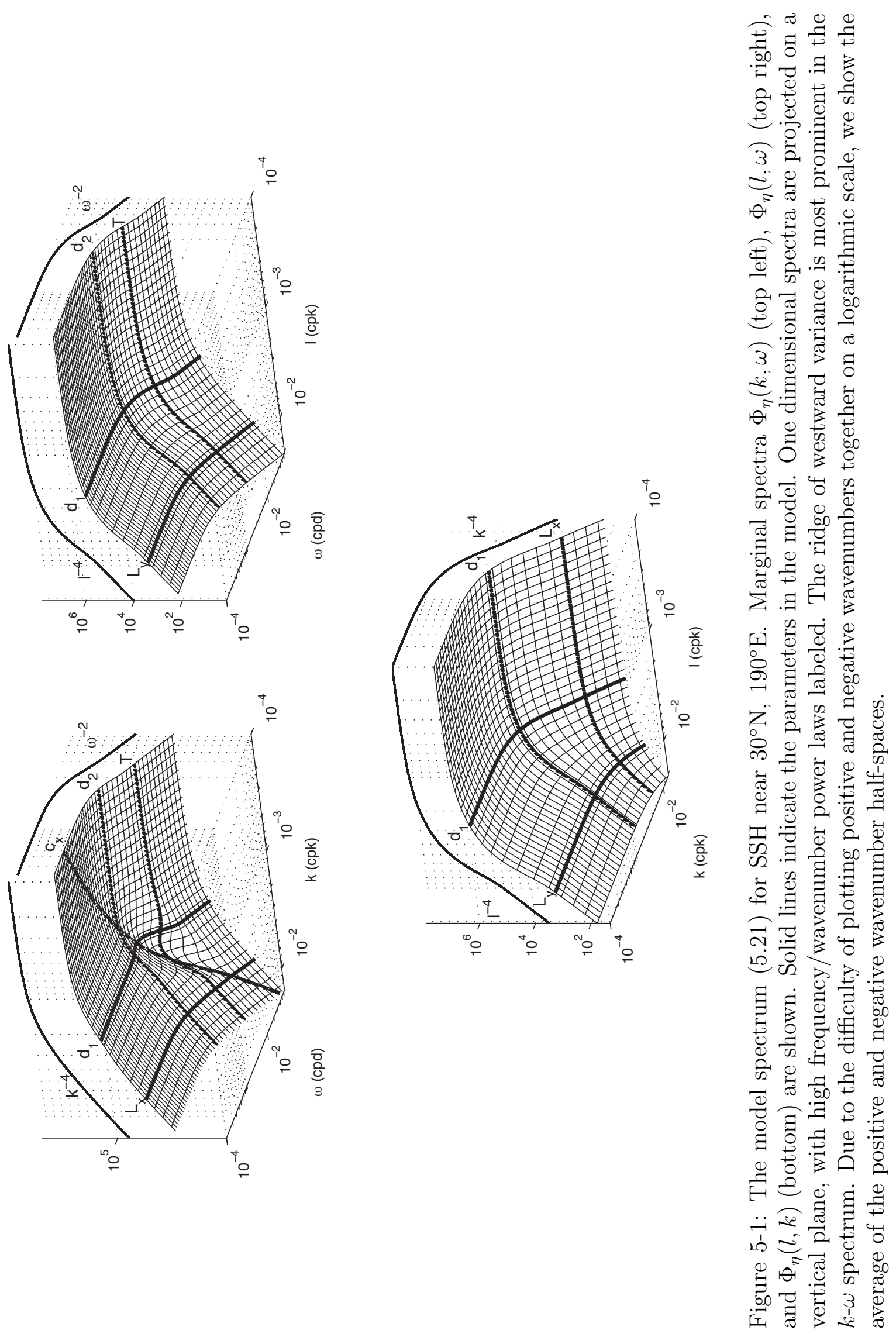
We consider briefly the high frequency and wavenumber limits of (5.21). As the wavenumber increases, the first term dominates and the spectrum approaches a power law in $k, l$, and $\omega$. Integrating over $l$ and $\omega$ while neglecting the exponential term gives the high zonal wavenumber spectrum:

$$
\begin{aligned}
\Phi_{\psi}(k ; \phi, \lambda) & =\int_{-\infty}^{\infty} \int_{0}^{\infty} \Phi_{\psi}(k, l, \omega ; \phi, \lambda) \mathrm{d} \omega \mathrm{d} l \\
& =\frac{\pi^{3 / 2} \Gamma(n-1 / 2)}{4 \Gamma(n)} d_{1}^{2 n} d_{2}\left(k^{2}+d_{1}^{2}\right)^{-n+1 / 2}
\end{aligned}
$$

with a similar expression for $\Phi_{\psi}(l ; \phi, \lambda)$. The constant $n$ sets the high frequency and wavenumber spectral slope. For simplicity, we use a constant $n=5 / 2$. The resulting one-dimensional wavenumber spectral slope is $k^{-4}$ for $\mathrm{SSH}$ and $k^{-2}$ kinetic energy.

High-wavenumber spectral slopes appear vary geographically, but the true values are quite uncertain. Stammer [1997] concluded, based on filtered along-track wavenumber spectra from altimetry, that SSH spectra displayed a remarkably universal $k^{-4}$ power law at wavelengths shorter than $400 \mathrm{~km}$. Xu and Fu [2012] used different estimates of the altimeter noise, and estimated spectral slopes varying from $k^{-4.5}$ in high energy regions to $k^{-2}$ in low energy subtropics, and $k^{-1}$ in the tropics. Meanwhile, in situ measurements give different results. Wavenumber spectra from shipboard ADCP in the Gulf Stream suggest a $k^{-3}$ spectral slope for kinetic energy, implying $k^{-5}$ for SSH [Wang et al., 2010]. In the central North Pacific $\left(25-35^{\circ} \mathrm{N}\right.$, $\left.140^{\circ} \mathrm{W}\right)$, spectral slopes from shipboard ADCP are close to $k^{-2}$ for velocity, implying $k^{-4}$ for SSH (J. Callies 2012, private communication). Both of the in situ spectral slope estimates are steeper than altimeter derived estimates in their respective regions. Given this uncertainty, we do not see a significant benefit to proposing a variable $n$ at this time. More accurate spectral slope estimates in the future could be used to revise this aspect of the model spectrum.

The choice of constant $n=5 / 2$ is unlikely to be the leading cause of error for our purposes. The applications that we propose for the model spectrum are primarily sensitive to the correlation lengths. Observed wavenumber spectra can broadly be described as a low wavenumber plateau transitioning to a steep power law at higher 
wavenumber. For such a spectrum, the dominant wavelengths are more sensitive to the wavelength of the transition than to the slope of the power law ${ }^{3}$. As discussed in the introduction, high wavenumber spectral slopes are important for distinguishing between various ocean turbulence theories. However, distinguishing between such theories is beyond the scope of this thesis.

At high frequency, the first term in (5.21) again dominates, resulting in a power law for frequency. Integrating over $k$ and $l$ while neglecting the exponential term gives the frequency spectrum:

$$
\begin{aligned}
\Phi_{\psi}(\omega ; \phi, \lambda) & =\int_{-\infty}^{\infty} \int_{-\infty}^{\infty} \Phi_{\psi}(k, l, \omega ; \phi, \lambda) \mathrm{d} k \mathrm{~d} l \\
& =\frac{\pi d_{1}^{2} d_{2}^{2}}{n-1}\left(\omega^{2}+d_{2}^{2}\right)^{-1}
\end{aligned}
$$

At high frequency, the spectral slope is $\omega^{-2}$ for $\mathrm{SSH}$, and the same for kinetic energy.

The most important parameters in the model spectrum (5.21) are $L_{x}, L_{y}$, and T, which set the dominant wavelengths and periods. Jacobs et al. [2001] estimated zonal wavelength, meridional wavelength, and period by fitting an exponential to the binned covariance function estimated from altimetry ${ }^{4}$. In fact, similar values are used in the objective mapping procedure for the AVISO gridded altimetry product [Le Traon et al., 2003]. Rather than estimating these parameters anew, we base ours on the estimates by Jacobs et al. [2001]. Specifically,

$$
\begin{aligned}
& L_{x}=50+250 \exp \left\{-\frac{\phi^{2}}{750}\right\} \mathrm{km} \\
& L_{y}=50+200 \exp \left\{-\frac{\phi^{2}}{600}\right\} \mathrm{km} \\
& T=20+150 \sin ^{2}(2 \phi) \text { days }
\end{aligned}
$$

where $\phi$ is latitude in degrees.

\footnotetext{
${ }^{3}$ Zang [2000] gives a more detailed description of the impact of uncertainty in spectral slope on correlation wavelength.

${ }^{4}$ The existing estimates of the parameters $L_{x}, L_{y}$, and $T$ by Jacobs et al. [2001] are a strong motivation for using the exponential in (5.21).
} 
The parameters $d_{1}$ and $d_{2}$ control the transition from a low frequency/wavenumber plateau (where the model spectrum is the sum of the power law and exponential terms) to a high frequency/wavenumber power law (where the exponential becomes negligible). In order for the model spectrum to transition smoothly between the low and high frequency/wavenumber regimes, these parameters must depend on $L_{x}, L_{y}$, and $T$. A reasonable fit is achieved with

$$
\begin{aligned}
& d_{1}=\frac{1}{3 L_{x}} \\
& d_{2}=\frac{1}{3 T}
\end{aligned}
$$

The parameter $c_{x}$ controls the dominant zonal phase speed in the model spectrum. We use the long Rossby wave phase speed for a rough-bottom ocean, as calculated in Ch. 4. While a dominant meridional phase speed can always be estimated, they are typically much smaller than the zonal phase speed [Jacobs et al., 2001; Fu, 2009]. Further, the there is generally equal variance propagating northward and southward (Ch. 2), rendering the value of the meridional phase speed relatively unimportant. Hence, we set $c_{y}=0$.

Figure 5-3 shows the zonal wavenumber-frequency spectra from observations and the model (5.21) near $30^{\circ} \mathrm{N}, 190^{\circ} \mathrm{E}$. The model spectrum includes a ridge of westward variance qualitatively similar to the observed spectrum, but there are many differences. Perhaps most notable, the range of spectral power in the observed spectrum is an order of magnitude smaller than the range of power in the model spectrum. This discrepancy is largely due to the fact that the observed spectrum falls off faster at high wavenumber/frequency, making spectral power more concentrated along the ridge of westward variance. However, the model spectrum presented here gives a better fit to other in situ observations (discussed in the following sections) than would a model that matched the altimetric spectrum closely. Based on the differences between spectra from gridded and along-track altimetry discussed in Ch. 2, we choose not to attempt to fit the spectrum from gridded altimetry exactly.

The model spectrum (5.21) is intended to approximate the observed spectrum for 


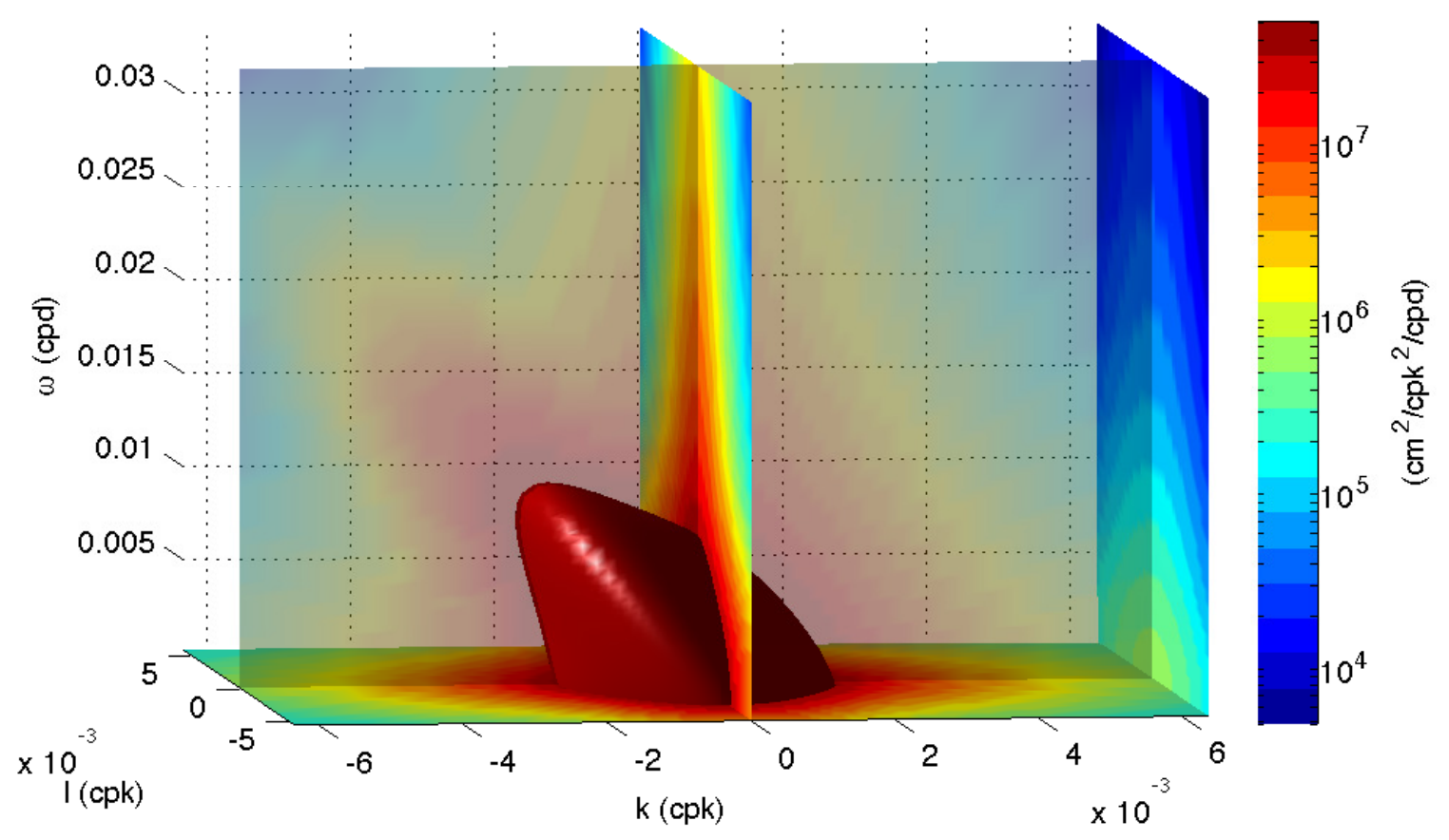

Figure 5-2: Same as Fig. 2-7, but for the model spectrum (5.21) for SSH near $30^{\circ} \mathrm{N}$, $190^{\circ} \mathrm{E}$.
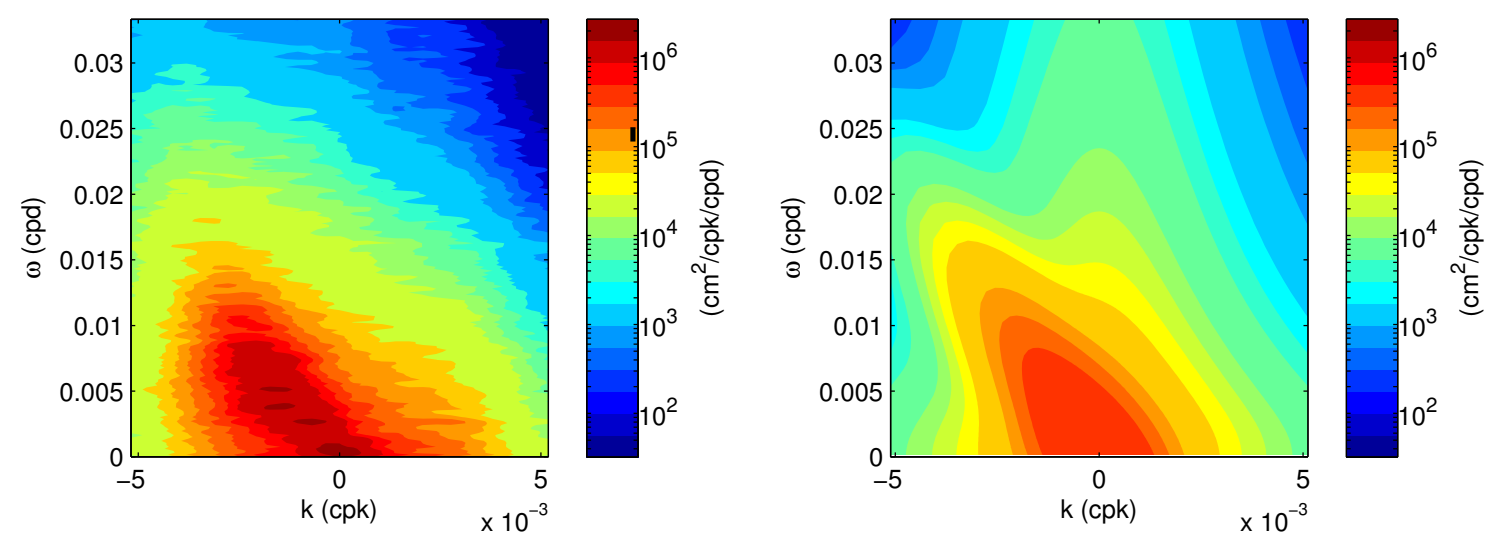

Figure 5-3: The zonal wavenumber-frequency spectrum of SSH near $30^{\circ} \mathrm{N}, 190^{\circ} \mathrm{E}$ from altimetry (left) and the model spectrum (right). 
periods between 30 days and about 20 years, and wavelengths between $200 \mathrm{~km}$ and $10,000 \mathrm{~km}$. These limits are primarily set by the duration and spatial resolution of the gridded altimetric product [Chelton et al., 2011]. The important question of the behavior of the frequency spectrum as $\omega$ approaches zero is beyond the scope of this work, but has been discussed elsewhere [Wunsch, 2010, and references therein]. The model spectrum presented here becomes white in frequency as $\omega \rightarrow 0$.

\subsubsection{Vertical structure}

Most of what is known about the vertical structure of variability from observations is based on Wunsch [1997]. The basic inference was that about 50\% of the watercolumn kinetic energy is in the barotropic mode, about $40 \%$ in the first baroclinic mode, and the remainder in higher baroclinic modes. The modes were defined as the basic flat-bottom resting ocean Rossby wave modes. However, Wunsch [1997] found evidence of coupling between the modes, such that the total kinetic energy was less than the sum of the energy in each mode. Mode coupling inferred from moorings increased the kinetic energy in some places and decreased it in others. In contrast, the barotropic and first baroclinic modes in the ECCO2 model (Ch. 3) are strongly coupled with a phase relation that increases the surface kinetic energy almost everywhere. Such coupling suggests that the flat-bottom resting ocean modes are not the correct dynamical modes.

The observed mode coupling may be due to a number of dynamical processes. The vertical structure of Rossby wave modes can be affected by mean flow [Killworth and Blundell, 2004, 2005], surface forcing [Müller and Frankignoul, 1981], surface buoyancy anomalies as in the SQG theory [Lapeyre and Klein, 2006], bottom roughness [Bobrovich and Reznik, 1999; Tailleux and McWilliams, 2001], as well as other physics [Scott and Furnival, 2012].

Based on the results of Ch. 3 and 4, we use the rough-bottom dynamical modes to describe the vertical structure of variability. While the partition between the modes is likely to vary geographically, we use a constant partition of $1 / 2: 1: 1 / 5: 1 / 5: 1 / 5$ for the barotropic through fourth baroclinic rough-bottom modes. The rough-bottom 
first baroclinic mode is approximately a linear combination of the flat-bottom first baroclinic and barotropic modes. Hence, the use of the rough-bottom modes is an attempt to account for the apparent mode coupling when vertical profiles of horizontal velocity are decomposed into the flat-bottom modes.

Other dynamical processes are likely important in various regions. In the tropical Pacific, for example, the observed frequency-wavenumber spectrum is consistent with Rossby waves affected by the zonal mean flow [Farrar and Weller, 2006]. Strong mean flow in the western boundary current regions, near the equator, and in the ACC clearly impact the observed spectrum.

\subsection{Comparison with observations}

In this section, we compare the various observed frequency and wavenumber spectra with our model spectrum (5.21) using (5.17) and the characteristic functions (5.8)$(5.14)$.

\subsubsection{Global pattern}

One useful way to characterize the model spectrum is in terms of its moments, as we did for the observed spectrum in Ch. 2. Define

$$
\begin{aligned}
\left\langle\omega^{q}\right\rangle & =\int_{0}^{\infty} \omega^{q} \Phi_{\psi}(\omega) \mathrm{d} \omega / \int_{0}^{\infty} \Phi_{\psi}(\omega) \mathrm{d} \omega \\
\left\langle k^{q}\right\rangle & =\int_{0}^{\infty} k^{q}\left[\Phi_{\psi}(k)+\Phi_{\psi}(-k)\right] \mathrm{d} k / \int_{0}^{\infty}\left[\Phi_{\psi}(k)+\Phi_{\psi}(-k)\right] \mathrm{d} k \\
\left\langle l^{q}\right\rangle & =\int_{0}^{\infty} l^{q}\left[\Phi_{\psi}(l)+\Phi_{\psi}(-l)\right] \mathrm{d} l / \int_{0}^{\infty}\left[\Phi_{\psi}(l)+\Phi_{\psi}(-l)\right] \mathrm{d} l
\end{aligned}
$$

for integer $q$. For $k$ and $l$, we consider the moments of the spectrum averaged over positive and negative wavenumber. Maps of the dominant phase speed and the inverse of the first moments of frequency and wavenumber are shown (Fig. 5-4). The dominant phase speed is $c_{x}$ used in (5.21). The dominant period increases from roughly 100 days near the equator to 200 days at high latitude. The dominant zonal wavelength 

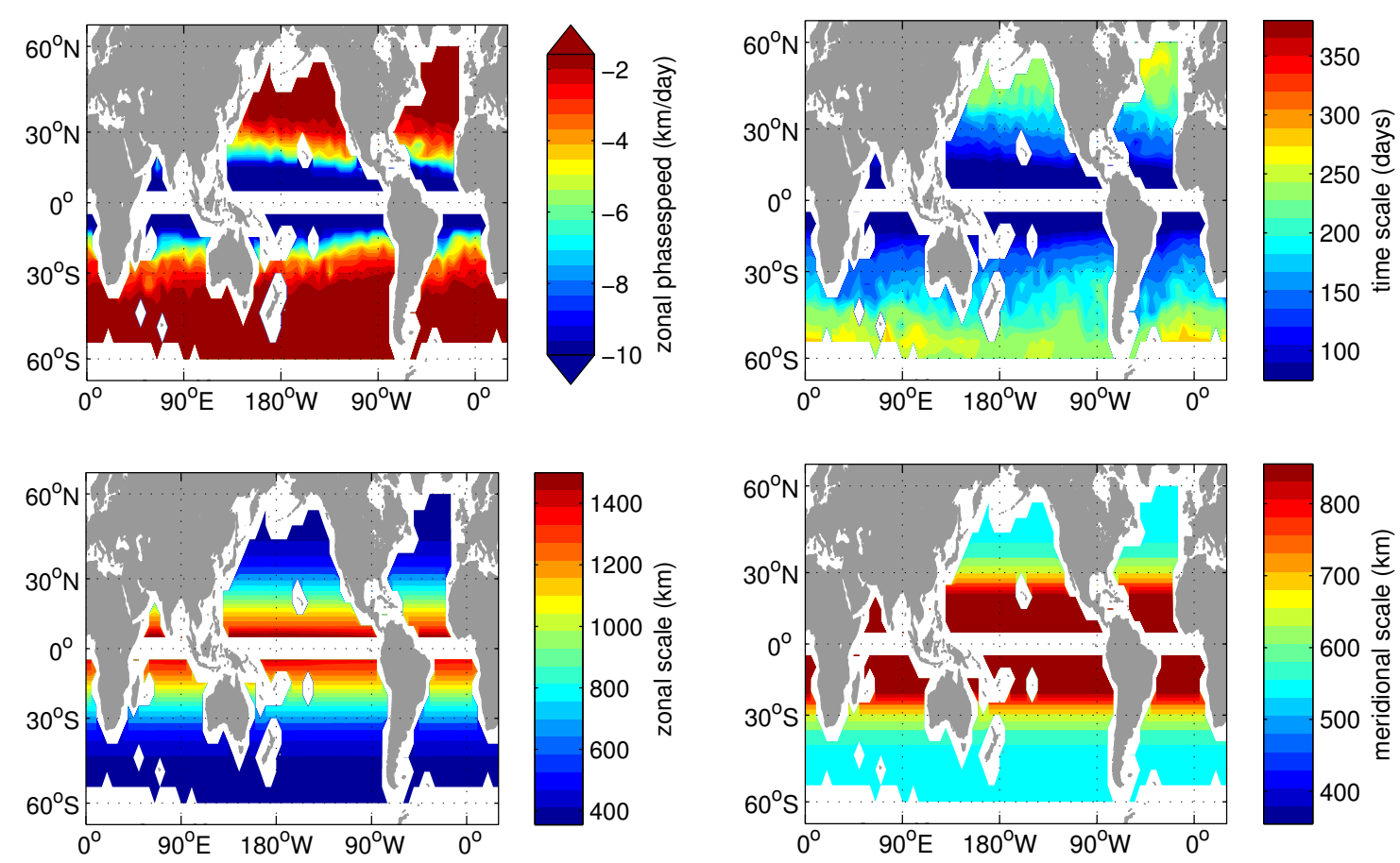

Figure 5-4: Global characteristics of the model spectrum (5.21). Dominant zonal phase speed estimated from the peak of the $k$ - $\omega$ spectrum (top left), dominant period (top right), zonal wavelength (bottom left), and meridional wavelength (bottom right) based on the first moment of the associated one-dimensional spectra. Compare to Fig. 2-17.

decreases with latitude, from $1500 \mathrm{~km}$ to $350 \mathrm{~km}$. The meridional wavelength has a slightly smaller range, decreasing with latitude from $1300 \mathrm{~km}$ to $550 \mathrm{~km}$.

Figure 5-4 can be compared with Fig. 2-17. Dominant wavelengths estimated from altimetry are consistently larger than the first moment of the model spectrum. However, the objective mapping process for the gridded altimetry significantly attenuates small wavelengths, as discussed in Ch. 2, biasing the estimated dominant wavelength high by an unknown amount.

Figure 5-4 does reveal two clear shortcomings of the model spectrum. First, the model spectrum does not capture the long periods estimated from the altimetric spectrum. Second, the model spectrum does not capture the differences between the North Atlantic and Pacific, which are likely related to differences between the Gulf Stream and Kuroshio. 


\subsubsection{Along-track altimetry}

While the gridded altimetry provides a useful tool for studying the three-dimensional SSH spectrum and global variations in the spectrum, the objective mapping procedure significantly alters the shape of the spectrum. Therefore, we compare the model spectrum with the TOPEX/POSEIDON along-track altimetry, rather than the gridded altimetry product.

Observed and modeled wavenumber spectra are shown for two locations in the North Pacific (Fig. 5-5), a high energy region centered at $35^{\circ} \mathrm{N}, 166^{\circ} \mathrm{E}$ and a relatively low energy region centered at $35^{\circ} \mathrm{N}, 222^{\circ} \mathrm{E}$. In both regions, the model spectrum captures the general shape of the observed spectrum at wavelengths larger than 100 $\mathrm{km}$. Our model spectrum has a constant high-wavenumber spectral slope of $k^{-4}$ for SSH. In the high energy region, the spectral slope in the 100-200 km wavelength band is close to $k^{-4}$, but the spectral slope is significantly flatter in the low energy region, closer to $k^{-2}$. At smaller wavelengths, the spectral slope flattens significantly. Much of the high wavenumber tail is due to noise, but the extent to which the altimetric wavenumber spectrum is contaminated by noise is a matter of debate [Stammer, 1997; $X u$ and $F u, 2012]$.

Figure 5-5 shows observed and modeled frequency spectra for the same locations, $35^{\circ} \mathrm{N}, 166^{\circ} \mathrm{E}$ and $35^{\circ} \mathrm{N}, 222^{\circ} \mathrm{E}$. Whereas the wavenumber spectral slopes are clearly different between the two regions, the frequency spectral slopes near 100 days are more difficult to determine. Both spectra transition to relatively flat spectral slopes in the high-frequency tail. Based on comparison with in situ frequency spectra below, we suspect that the high-frequency tail may include a significant contribution from noise or aliasing of higher frequency motion. The broad spectral peak near $6.8 \times 10^{-4} \mathrm{cpd}$ is not significant at the $95 \%$ confidence level.

\subsubsection{Moored kinetic energy spectrum}

Figure 5-6 shows observed and modeled kinetic energy spectra from a mooring at $33^{\circ} \mathrm{N}, 232^{\circ} \mathrm{E}$ in the North Pacific. Instrument depths are $77 \mathrm{~m}, 431 \mathrm{~m}, 719 \mathrm{~m}$, and 

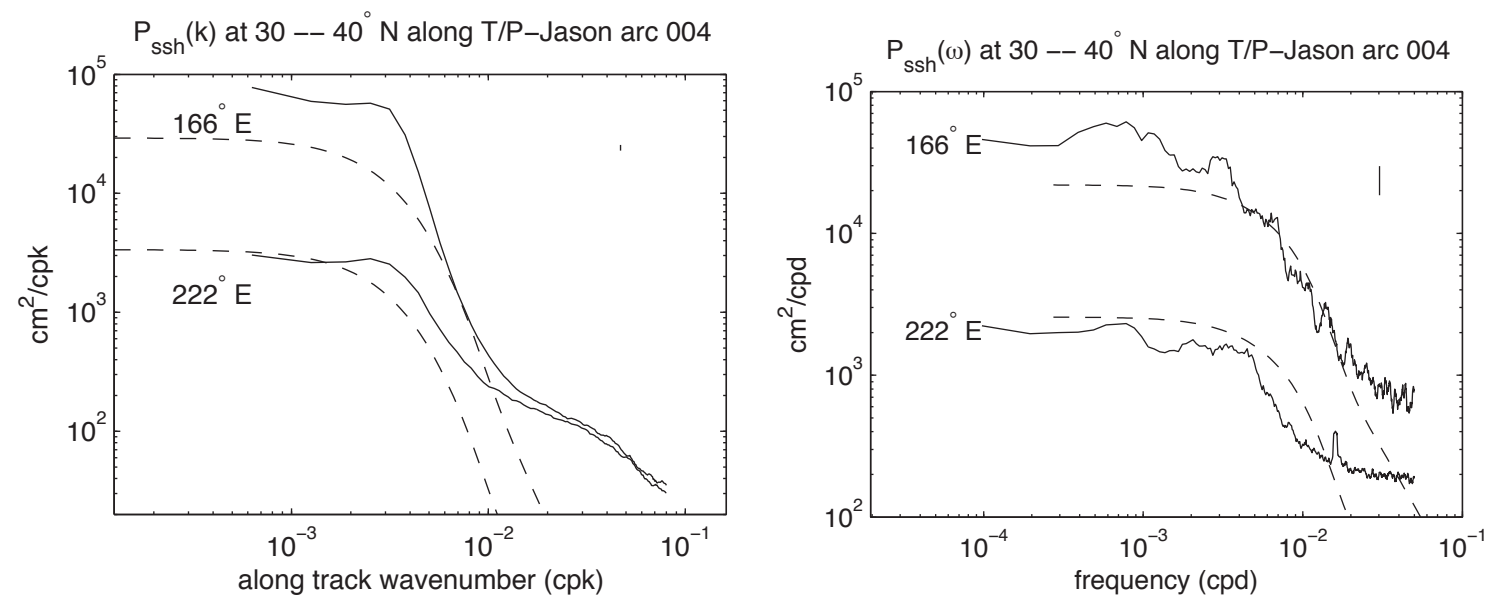

Figure 5-5: Along-track wavenumber (left) and frequency (right) spectra from tracks spanning $30^{\circ} \mathrm{N}$ to $40^{\circ} \mathrm{N}$ at the indicated longitude (solid line). Dashed line shows the model spectrum at each location. Vertical bars indicate the $95 \%$ confidence interval.

$1418 \mathrm{~m}$, and the total water depth was $4524 \mathrm{~m}$. Except for the shallowest instrument, the model spectrum follows the observed spectrum very closely, and is within the estimated uncertainty of the latter. In particular, the model spectrum captures the transition from low-frequency plateau to high-frequency $\omega^{-2}$ power law.

As the period approaches one day, the observed spectrum deviates from the model. The observed spectrum transitions smoothly into the internal wave regime, which is not included in our model (Fig. 5-7). Spectral peaks at the inertial frequency, $M_{2}$ tidal frequency, and the $\omega^{-2}$ internal wave continuum described by Garrett and Munk [1972, 1975] dominate the high frequency portion of the spectrum.

\subsubsection{Moored temperature spectrum}

Observed and model temperature spectra from the same instruments discussed above are shown in Fig. 5-8. The observed spectrum exceeds the model spectrum by about a factor of 5 at all depths. One possible cause of this discrepancy is a breakdown in the assumption that temperature fluctuations are generated only by vertical advection of the mean temperature gradient. This assumption neglects horizontal advection of temperature gradients. However, for the central Pacific mooring considered here, horizontal temperature gradients are small and vertical advection accounts for most 

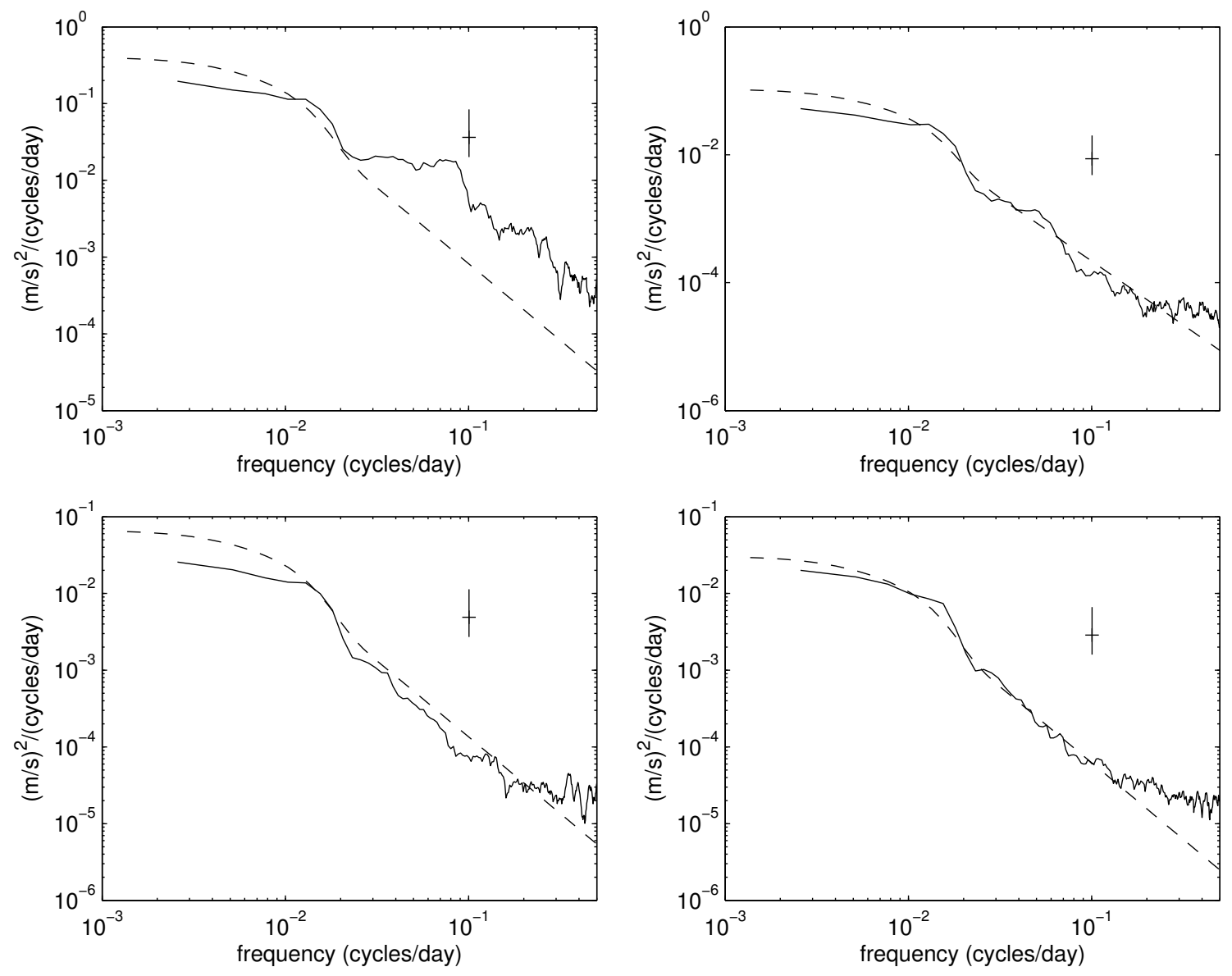

Figure 5-6: Observed (solid) and modeled (dashed) frequency spectra of kinetic energy from moored instruments at $33^{\circ} \mathrm{N}, 232^{\circ} \mathrm{E}$. Instrument depths are $77 \mathrm{~m}$ (top left), $431 \mathrm{~m}$ (top right), $731 \mathrm{~m}$ (bottom left), and $1481 \mathrm{~m}$ (bottom right).

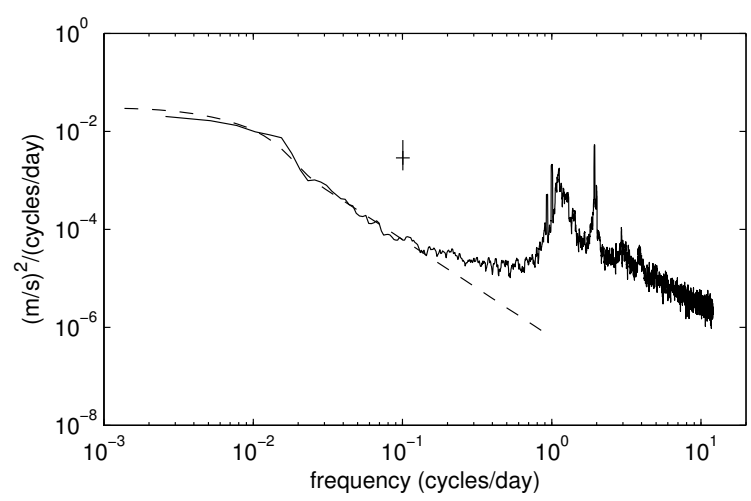

Figure 5-7: Observed (solid) and modeled (dashed) frequency spectra of kinetic energy from moored instruments at $33^{\circ} \mathrm{N}, 232^{\circ} \mathrm{E}, 1481 \mathrm{~m}$ deep, as in Fig. 5-6, but now expanded to show the internal wave regime. The spectral peaks are at the inertial and $M_{2}$ tidal frequencies. 
of the temperature variability below the mixed layer [Forget and Wunsch, 2007]. A second possible cause is an error in the climatological temperature gradient. However, differences between the climatological temperature gradient used and those estimated from the mooring data are too small to account for the error.

The poor fit of the model spectrum amplitude to observations arises from uncertainty in the vertical structure of the model. From (5.14), the amplitude of the model temperature spectrum at depth $z$ is the product of two derivatives: $\partial \theta_{0} / \partial z$ and $G_{n}(z) \sim \mathrm{d} F_{n}(z) / \mathrm{d} z$. Errors in the horizontal velocity vertical mode shape $F_{n}(z)$ are amplified in the vertical velocity mode shape. While we have focused on the effect of rough topography, which alters the mode amplitude at depth, Aoki et al. [2009] showed that mean flow can alter the Rossby wave vertical modes near the thermocline, typically increasing $\mathrm{d} F_{n}(z) / \mathrm{d} z$ there. This error in the vertical mode shapes appears to be the cause of the discrepancy between model and observed temperature spectra.

Zang and Wunsch [2001] found much better agreement between their model and observed temperature spectra. However, they used an exponential buoyancy frequency profile with specified e-folding depth to compute the vertical modes. Setting the e-folding depth introduced another tunable parameter, allowing a better fit between model and observed spectra. Here, we compute the buoyancy frequency profile from climatological hydrographic profiles.

We note that the model spectrum does have a similar shape to the observed moored temperature spectrum, including the frequency at which the spectrum transitions from low-frequency plateau to high-frequency power law, and the $\omega^{-2}$ spectral slope. The frequency spectral slope for moored kinetic energy and moored temperature both appear close to $\omega^{-2}$. This is an important constraint on the model spectrum; a model of the form $\Phi_{\psi} \sim\left(k^{2}+l^{2}+\omega^{2}\right)^{-3}$ would predict spectral slopes of $k^{-4}$ and $\omega^{-4}$ for SSH and temperature but $k^{-2}$ and $\omega^{-2}$ for kinetic energy ${ }^{5}$. Our model spectrum (5.21) predicts that kinetic energy and temperature have the same

\footnotetext{
${ }^{5} \mathrm{~A}$ model of the form $\Phi_{\psi} \sim\left(k^{2}+l^{2}+\omega^{2}\right)^{-3}$ was considered, and gave a qualitatively better fit to the $k-\omega$ spectrum from altimetry. However the model was rejected due to a poor fit to the observed temperature spectrum.
} 

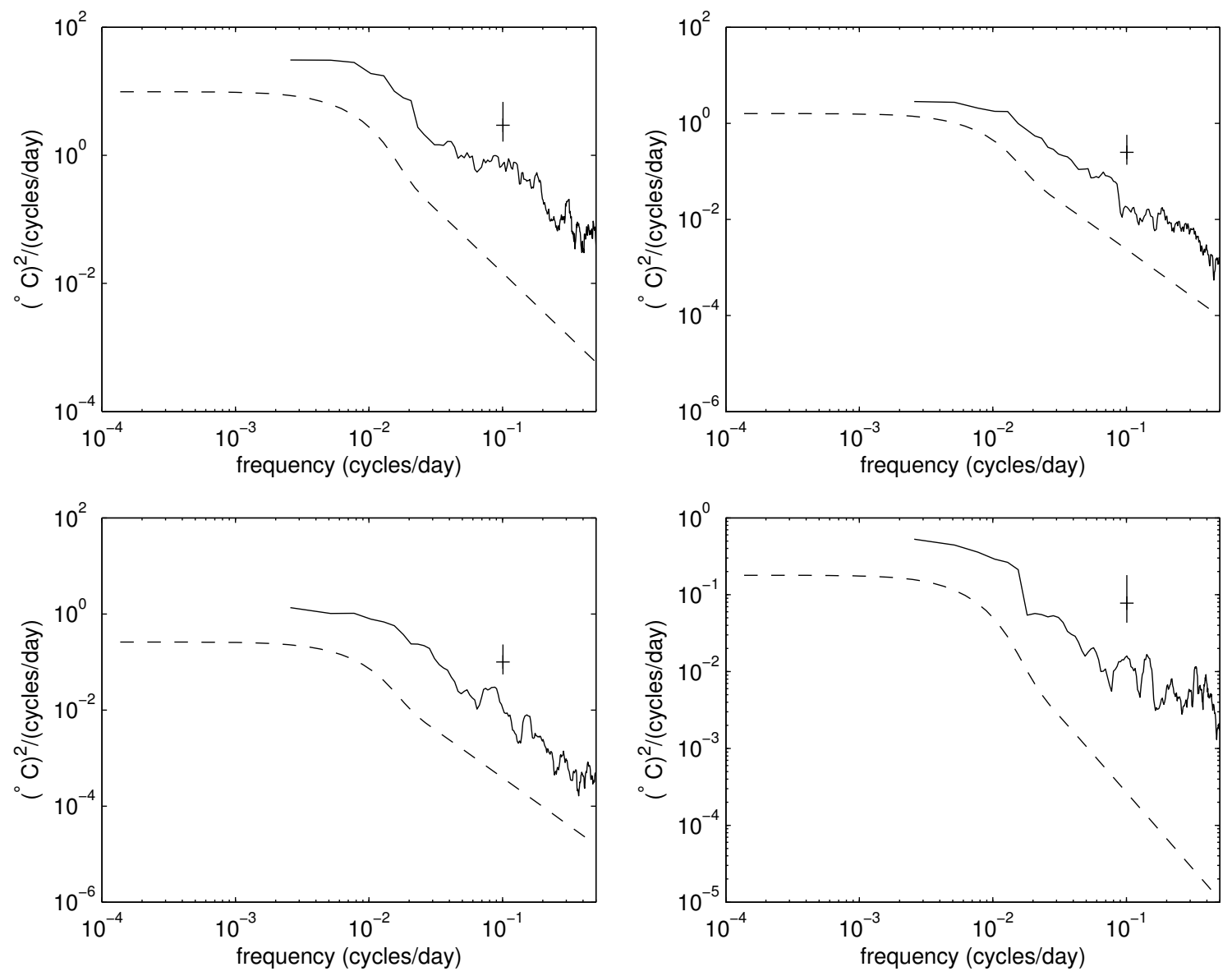

Figure 5-8: Observed (solid) and modeled (dashed) frequency spectra of potential temperature from moored current meters at $33^{\circ} \mathrm{N}, 232^{\circ} \mathrm{E}$. Instrument depths are $77 \mathrm{~m}$ (top left), $431 \mathrm{~m}$ (top right), $718 \mathrm{~m}$ (bottom left), and $1481 \mathrm{~m}$ (bottom right). 


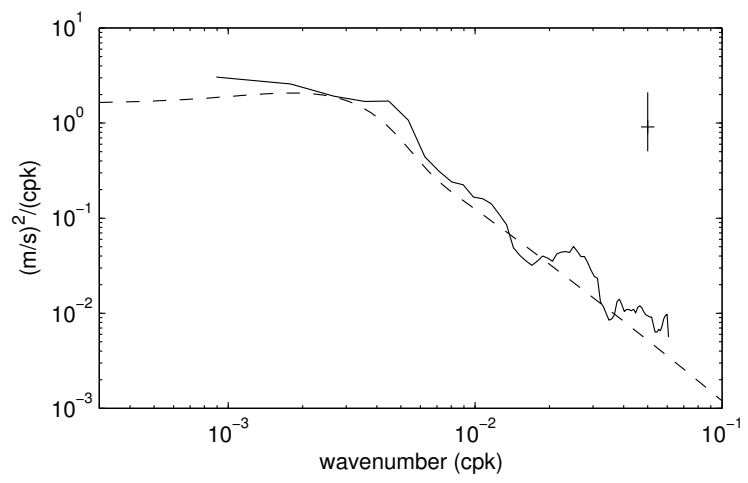

Figure 5-9: Observed (solid) and modeled (dashed) wavenumber spectra of kinetic energy from shipboard ADCP. The transect spans $20^{\circ}-30^{\circ} \mathrm{N}$ at $180^{\circ} \mathrm{E}$ at $100 \mathrm{~m}$ depth. The high wavenumber spectral slope for the model is $k^{-2}$.

frequency spectral slope.

\subsubsection{Towed velocity spectrum}

Next, we consider wavenumber spectrum of kinetic energy from in situ data (Fig. 5-9). Such data are difficult to obtain, but the best approximation comes from shipboard ADCP measurements made during a continuous transit. If the transit is sufficiently fast, temporal changes in the flow can be neglected and we interpret the results as representing the spatial variability. The data considered here are from a meridional transect as part of WOCE section $\mathrm{P} 14 \mathrm{~N}\left(180^{\circ} \mathrm{E}, 20^{\circ}-30^{\circ} \mathrm{N}\right)$ at $100 \mathrm{~m}$ and span 5 days [Roden, 2005].

The observed high-wavenumber spectral slope is close to the $k^{-2}$ predicted by the model. This spectral slope for kinetic energy implies $k^{-4}$ for SSH, significantly steeper than the $k^{-2}$ for SSH from altimetry at $222^{\circ} \mathrm{E}$ (Fig. 5-5) and the $k^{-3}$ at $180^{\circ} \mathrm{E}$ estimated by $X u$ and $F u$ [2012]. This discrepancy again highlights the likelihood that altimetric wavenumber spectra have significant noise contamination in low energy regions. 


\subsection{Applications}

At last, we arrive at the primary motivation for this thesis; we illustrate the use of our proposed model spectrum for oceanographic research. Zang [2000] discussed a number of applications for such a model. At the core of many applications is the fact that the Fourier transform of the spectrum is the covariance function, which is useful in objective mapping and interpolation. Second, the model can be used to estimate the variability of volume flux across a or heat content, and hence the uncertainty in trends in those quantities. Third, the model can be used in the design of observational networks by predicting the uncertainty that will result from a particular sampling pattern. Finally, Zang [2000] discussed the differences between Lagrangian and Eulerian correlation based on the model spectrum. Wunsch [2008] expanded on these applications, discussing the uncertainty in transport trends estimated by a pair of moorings spanning the North Atlantic. The proposed model spectrum has another use: by making a concrete statement about the spectrum of low-frequency oceanic variability, we pose a challenge to theoreticians to explain the spectrum.

\subsubsection{Correlation function}

By the Wiener-Khinchin theorem, the Fourier transform of the power spectrum is the covariance function ${ }^{6}$ :

$$
\operatorname{Cov}\left(r_{x}, r_{y}, \tau\right)=\int_{-\infty}^{+\infty} \int_{-\infty}^{+\infty} \int_{-\infty}^{+\infty} \Phi_{\chi}(k, l, \omega) e^{i 2 \pi\left(k r_{x}+l r_{y}+\omega \tau\right)} \mathrm{d} k \mathrm{~d} l \mathrm{~d} \omega
$$

\footnotetext{
${ }^{6}$ Since Ch. 2, we have only considered the positive-frequency half of the spectrum because, for real data, $\Phi(k, l, \omega)=\Phi(-k,-l,-\omega)$ rendering the negative-frequency half of the spectrum redundant. However, when computing the Fourier transform it is mathematically convenient to retain the negative-frequency half of the spectrum. Using the two-sided spectrum makes it a Hermitian function, and the Fourier transform of a Hermitian function is real, as the covariance function for real data must be.
} 
for separation $r_{x}, r_{y}$, and $\tau$ in the zonal, meridional, and temporal directions, respectively. Normalizing by the signal variance gives the correlation function,

$$
\operatorname{Cor}\left(r_{x}, r_{y}, \tau\right)=\frac{\operatorname{Cov}\left(r_{x}, r_{y}, \tau\right)}{\sigma_{\chi}^{2}}
$$

where $\sigma_{\chi}^{2}$ is the variance of $\chi$. Thus far, we have not been able to find a closed form for the correlation function based on (5.21).

More commonly, we consider the correlation function as a function of separation in one direction only. In this case, the correlation is a function of separation distance only, sign of the separation becomes meaningless. That is, propagation direction cannot be determined from the one-dimensional correlation function. Here, we use the non-directional wavenumber spectra ${ }^{7}$

$$
\begin{aligned}
\Phi_{\chi}^{N D}(k) & =\frac{\Phi_{\chi}(k)+\Phi_{\chi}(-k)}{2} \\
\Phi_{\chi}^{N D}(l) & =\frac{\Phi_{\chi}(l)+\Phi_{\chi}(-l)}{2}
\end{aligned}
$$

The one-dimensional covariance functions for zonal, meridional, and temporal separation are

$$
\begin{aligned}
& \operatorname{Cov}\left(r_{x}\right)=\int_{-\infty}^{+\infty} \Phi_{\chi}^{N D}(k) \cos \left(2 \pi k r_{x}\right) \mathrm{d} k \\
& \operatorname{Cov}\left(r_{y}\right)=\int_{-\infty}^{+\infty} \Phi_{\chi}^{N D}(l) \cos \left(2 \pi k r_{y}\right) \mathrm{d} l \\
& \operatorname{Cov}(\tau)=\int_{-\infty}^{+\infty} \Phi_{\chi}^{N D}(\omega) \cos (2 \pi k \tau) \mathrm{d} \omega
\end{aligned}
$$

The one-dimensional correlation functions of temperature as a function of zonal, meridional, and temporal separation are shown in Fig. 5-10. Here, we consider the model spectrum at $30^{\circ} \mathrm{N}, 190^{\circ} \mathrm{E}$. The e-folding wavelength is $125 \mathrm{~km}$ for zonal separation, $110 \mathrm{~km}$ for meridional separation, and 40 days for temporal separation.

\footnotetext{
${ }^{7}$ Mathematically, the two-sided directional wavenumber spectrum $\Phi(k)$ is not an even function, and so its Fourier transform will have an imaginary part. This causes difficulty for interpreting the result as the correlation function for real data.
} 
Our model spectrum (5.21) is not separable in frequency and wavenumber, implying that the spatial correlation function is a function of frequency and vice versa. The zonal correlation function at frequency $\omega$ is

$$
\operatorname{Cov}\left(r_{x} ; \omega\right)=\int_{-\infty}^{+\infty} \Phi_{\chi}^{N D}(k, \omega) \cos \left(2 \pi k r_{x}\right) \mathrm{d} k
$$

again written in terms of the non-directional spectrum

$$
\Phi_{\chi}^{N D}(k, \omega)=\left[\Phi_{\chi}(k, \omega)+\Phi_{\chi}(-k, \omega)\right] / 2
$$

The zonal correlation function falls off slightly faster at high frequency $\left(\omega=1 / 15\right.$ day $\left.^{-1}\right)$ than at low frequency $\left(\omega=1 / 20\right.$ years $\left.^{-1}\right)$ (Fig. 5-10). However, the difference in e-folding wavelength is only $80 \mathrm{~km}$.

The characteristic functions (5.8)-(5.14) allow only a few different shapes for the horizontal wavenumber-frequency $\operatorname{spectrum}^{8}: \tilde{p}, \tilde{\rho}, \zeta$, and $\theta$ all have the same shape as the streamfunction spectrum; $\tilde{u}$ and $\tilde{v}$ are multiplied by $k$ and $l$, respectively; and $\tilde{w}$ is multiplied by $\omega$. Variables with the same spectral shape will have the same correlation function.

Figure 5-11 shows the autocorrelation functions for zonal and meridional velocity as a function of spatial separation. Multiplying by $k^{2}$ reduces zonal correlation of meridional velocity and multiplying by $l^{2}$ reduces meridional correlation of zonal velocity. In the context of a velocity spectrum measured by shipboard ADCP, both of these correlation functions correspond to across track velocity, regardless of ship heading. In contrast, along track velocity correlation wavelengths (that is, zonal correlation of zonal velocity and meridional correlation of meridional velocity) are relatively long.

\footnotetext{
${ }^{8} G(z)$ and $F(z)$ control the vertical structure, and do not affect the horizontal wavenumberfrequency spectrum or the correlation function.
} 

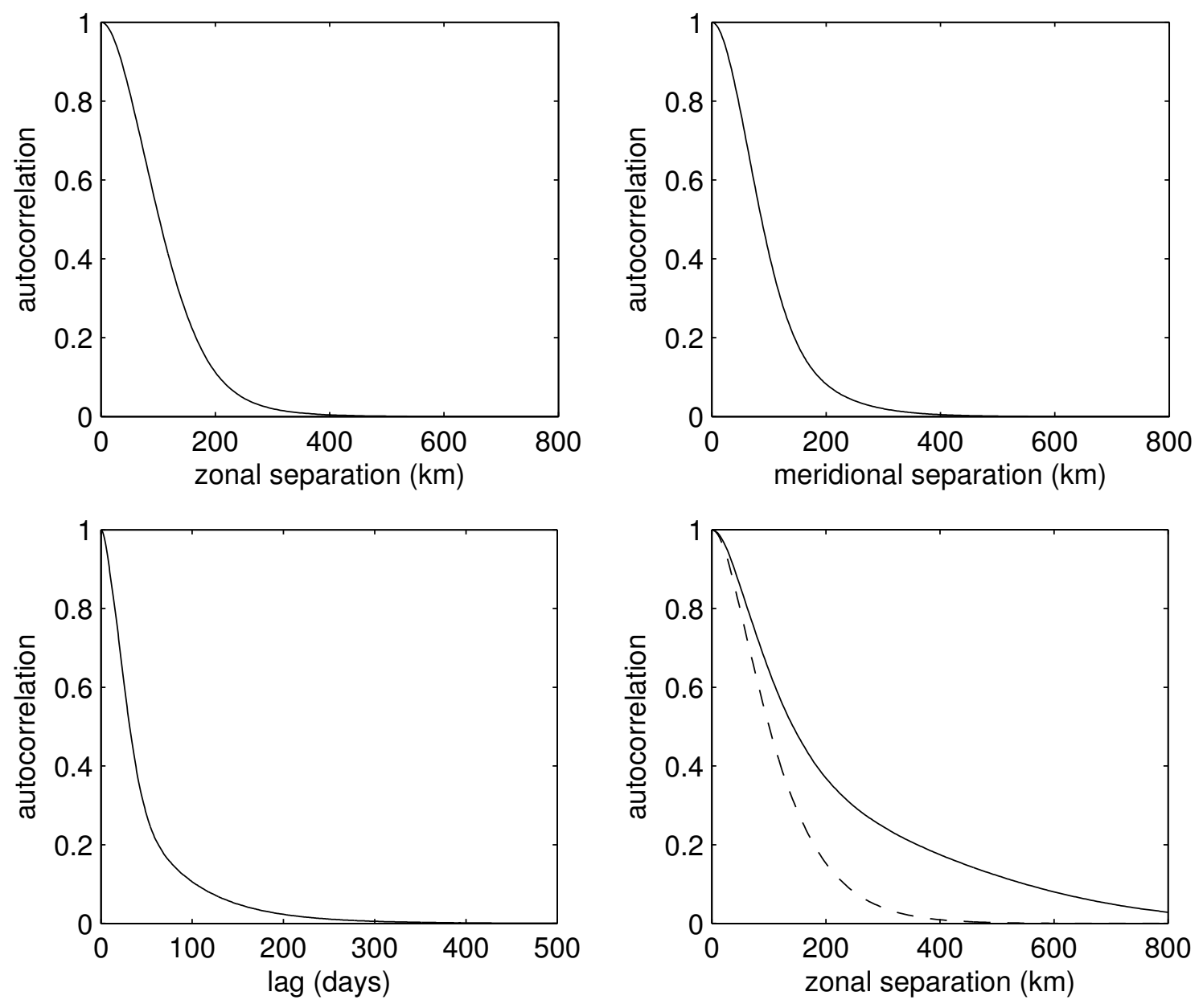

Figure 5-10: Autocorrelation function for temperature as a function of zonal separation (top left), meridional separation (top right), and temporal separation (bottom left) based on the model spectrum at $30^{\circ} \mathrm{N}, 190^{\circ} \mathrm{E}$. From the three-dimensional model spectrum (5.21), we can compute the spatial correlation at a particular frequency, $\operatorname{Cor}\left(r_{x} ; \omega\right)$ by $(5.42)$ (bottom right). The zonal correlation is slightly larger at low frequency $\left(1 / 20\right.$ years $^{-1}$; solid) than high frequency $\left(1 / 15\right.$ day $^{-1}$; dashed $)$. 

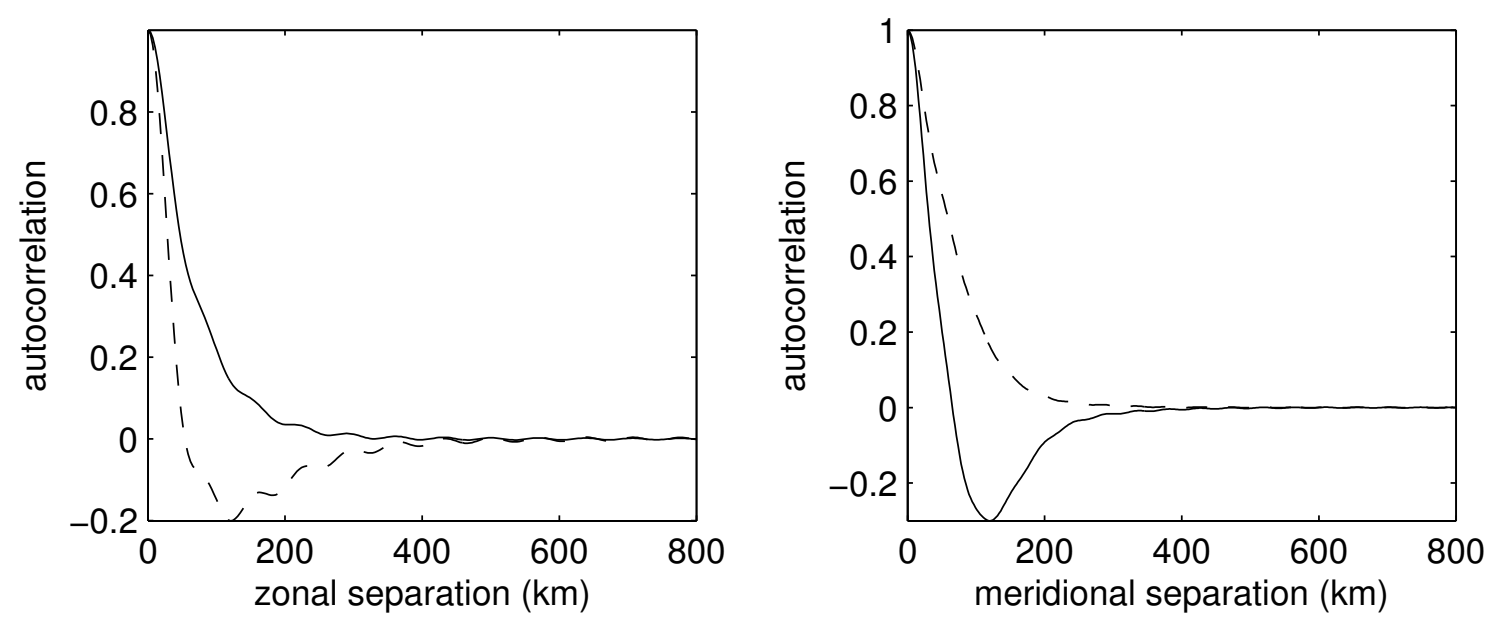

Figure 5-11: Autocorrelation function for zonal (solid) and meridional (dashed) velocity as a function of zonal separation (left), meridional separation (right), based on the model spectrum at $30^{\circ} \mathrm{N}, 190^{\circ} \mathrm{E}$.

\subsubsection{Trend detection and experiment design}

As noted in the introduction, the power spectrum is a convenient tool for estimating the uncertainty in the mean or trend from observations. A standard estimate of the uncertainty in $M$ independent, uncorrelated measurements of normally distributed random variable $\chi$ with variance $\sigma_{\chi}^{2}$ is $\sigma_{\chi} / \sqrt{M-1}$. However, correlation between the measurements can significantly change the uncertainty by reducing the number of degrees of freedom.

Given a set of observations $\mathbf{y}$ thought to be related through an unknown vector $\mathbf{x}$ by a linear model $\mathbf{E}$ by $\mathbf{y}=\mathbf{E x}+\mathbf{n}$ with noise $\mathbf{n}$, the minimum variance solution is [Wunsch, 2006, §2.7]

$$
\begin{aligned}
\tilde{\mathbf{x}} & =\left(\mathbf{R}_{x x}^{-1}+\mathbf{E}^{T} \mathbf{R}_{n n}^{-1} \mathbf{E}\right)^{-1} \mathbf{E}^{T} \mathbf{R}_{n n}^{-1} \mathbf{y} \\
\tilde{\mathbf{n}} & =\mathbf{y}-\mathbf{E} \tilde{\mathbf{x}} \\
\mathbf{P} & =\left(\mathbf{R}_{x x}^{-1}+\mathbf{E}^{T} \mathbf{R}_{n n}^{-1} \mathbf{E}\right)^{-1}
\end{aligned}
$$

where $\mathbf{P}$ is the error covariance matrix. Here, $\mathbf{R}_{x x}$ is the covariance matrix for the field to be estimated and represents prior information about the form of the solution, such as smoothness in space. This is generally quite uncertain and context dependent, so 
we will assume no prior information about the solution by setting $\left\|\mathbf{R}_{x x}\right\| \rightarrow \infty$. $\mathbf{R}_{n n}$ is the noise covariance matrix, and is our focus in this section. The diagonal elements of $\mathbf{R}_{n n}$ are the measurement variance, and the off diagonal elements represent the covariance between each observation and all other observations in $\mathbf{y}$.

In the context of large scale ocean circulation and climate change, low-frequency ocean variability constitutes "noise" obscuring the signal. This low-frequency variability is exactly what we seek to describe in the model spectrum (5.21). Below, we illustrate the use of the model spectrum for estimating the uncertainty in trends in ocean temperature.

Purkey and Johnson [2010] used repeat WOCE hydrographic sections to estimate trends in deep ocean temperature and heat content. Temperature trends were estimated for 24 ocean basins. Within each basin, each set of co-located temperature measurements were used to estimate the temperature trend at that location. Then the all temperature trends within a basin were averaged to give a basin-wide temperature trend. A typical basin might be sampled by one or two WOCE hydrographic sections, repeated 2-5 times, spanning a 5-20 year period. A typical hydrographic section spanned $2000 \mathrm{~km}$, with stations every $55 \mathrm{~km}$.

As a concrete example, we consider a single $2000 \mathrm{~km}$ section with stations every $55 \mathrm{~km}$ (37 stations), repeated four times at five year intervals. Our hypothetical section is centered at $30^{\circ} \mathrm{N}, 190^{\circ} \mathrm{E}$, and we are interested in the temperature trend at $2000 \mathrm{~m}$. The noise covariance matrix $\mathbf{R}_{n n}$ is formed by evaluating the covariance function (5.35) for each pair of measurements (37 stations $\times 4$ occupations in all). 
For a linear trend,

$$
\mathbf{E}=\left[\begin{array}{cc}
1 & t_{1} \\
1 & t_{2} \\
1 & t_{3} \\
1 & t_{4} \\
1 & t_{1} \\
1 & t_{2} \\
1 & t_{3} \\
1 & t_{4} \\
& \vdots
\end{array}\right] \text { station } 1
$$

For this sampling pattern, the $95 \%$ confidence interval for a trend is $3.6 \times 10^{-3}{ }^{\circ} \mathrm{C} /$ year. In contrast, reducing the sampling to two occupations increases the uncertainty to $1 \times 10^{-2}{ }^{\circ} \mathrm{C} /$ year, while increasing to seven occupations reduces the uncertainty to $1.6 \times 10^{-3}{ }^{\circ} \mathrm{C} /$ year.

The main results of Purkey and Johnson [2010] are in terms of heat flux through the $4000 \mathrm{~m}$ isobath, averaged over an ocean basin. For example, they report a heat flux of $0.03 \pm 0.01 \mathrm{~W} / \mathrm{m}^{2}$ for the North Pacific. At $4000 \mathrm{~m}$, the sampling pattern described above (37 stations $\times 4$ occupations in all) results in an uncertainty of $7 \times 10^{-5}{ }^{\circ} \mathrm{C} /$ year. This is much smaller than the uncertainty at $2000 \mathrm{~m}$ due to the reduced temperature variability at greater depth. For direct comparison with Purkey and Johnson [2010], we integrate the temperature trend over the volume of the ocean below $4000 \mathrm{~m}$ to estimate the heat flux through the $4000 \mathrm{~m}$ isobath:

$$
Q=\frac{1}{a(4000)} \int_{4000}^{\text {bottom }} \rho C_{p} \frac{\mathrm{d} \theta}{\mathrm{d} t} a \mathrm{~d} z
$$

where $a$ is the surface area of a given isobath and $C_{p}$ is the heat capacity. For a 
cylindrical ocean basin of constant depth and temperature trend ${ }^{9}$, this reduces to

$$
Q \approx \rho C_{p} \frac{\mathrm{d} \theta}{\mathrm{d} t}(\text { bottom }-4000 \mathrm{~m})
$$

The Purkey and Johnson [2010] heat flux trend and uncertainty for the North Pacific basin are based on approximately 7 hydrographic sections, reducing the uncertainty to $\sigma_{Q} / \sqrt{6}$. For our temperature trend uncertainty, the equivalent heat flux uncertainty is $0.005 \mathrm{~W} / \mathrm{m}^{2}$, about half the Purkey and Johnson [2010] estimate for the region. We attribute the discrepancy to the underestimate in the model spectrum temperature variance noted in $\S 5.3 .4$.

It is worth noting that the uncertainties resulting from the minimum variance estimate are independent of the data. Thus, the uncertainty can be predicted before any data is collected, assuming adequate estimates of the noise covariance exist. A priori uncertainty estimates should inform observational experiment design. For example, given the number of available instruments and sampling pattern, we can predict the time required to detect a statistically significant trend at of a given magnitude.

\subsubsection{Objective mapping of altimetry}

The model spectrum can also be applied to objective mapping, again through the associated covariance function. Objective mapping is used in many contexts, including analysis of the Mid Ocean Dynamics Experiments [Bretherton et al., 1976], interpolating average flow fields from floats [Gille, 2003], and mapping tracer distributions for mixing studies [Ledwell et al., 1998]. In all cases, objective mapping requires that a space-time covariance function be specified. As with the case of uncertainty estimates discussed above, the model spectrum (5.21) provides an estimate of the required covariance function in many contexts.

One important application of objective mapping is the interpolation of altimeter data to a regular grid for use in numerical models [Cummings, 2005] or as in the

\footnotetext{
${ }^{9}$ Purkey and Johnson [2010] perform the integral using detailed bathymetry and complicated basin shapes.
} 
AVISO gridded data product. In this case, the model spectrum discussed above provides an estimate of the signal covariance, rather than the noise covariance.

In the context of mapping altimetry data, the problem is to estimate the value of a scalar field $\mathbf{x}$ at locations $\tilde{r}$ given a set of observations $\mathbf{y}\left(r_{i}\right)$ at locations $r_{i}$, $i=1,2, \ldots N$. By the Gauss-Markov theorem, the minimum variance estimate is [Bretherton et al., 1976; Wunsch, 2006, §3.2]

$$
\begin{aligned}
& \tilde{\mathbf{x}}=\sum_{i=1}^{N} \sum_{j=1}^{N} \mathbf{R}_{x x}\left(\tilde{r}, r_{j}\right)\left\{\mathbf{R}_{x x}+\mathbf{R}_{n n}\right\}_{j i}^{-1} \mathbf{y} \\
& \tilde{\mathbf{n}}=\mathbf{y}-\tilde{\mathbf{x}} \\
& \mathbf{P}\left(\tilde{r}_{\alpha}, \tilde{r}_{\beta}\right)=\mathbf{R}_{x x}\left(\tilde{r}_{\alpha}, \tilde{r}_{\beta}\right)-\sum_{i=1}^{N} \sum_{j=1}^{N} \mathbf{R}_{x x}\left(\tilde{r}_{\alpha}, r_{j}\right)\left\{\mathbf{R}_{x x}+\mathbf{R}_{n n}\right\}_{j i}^{-1} \mathbf{R}_{x x}\left(r_{i}, \tilde{r}_{\beta}\right)
\end{aligned}
$$

In this case, the noise covariance $\mathbf{R}_{n n}$ is likely dominated by various measurement errors, as discussed by Le Traon et al. [1998].

The covariance $\mathbf{R}_{x x}$ plays an important role in the estimated field $\tilde{\mathbf{x}}$, and must be specified. Le Traon et al. [2003] use a covariance function of the form

$$
\operatorname{Cor}\left(r_{x}, r_{y}, \tau\right)=\left[1+a r+1 / 6(a r)^{2}-1 / 6(a r)^{3}\right] \times \exp \{-a r\} \exp \left\{-\tau^{2} / T^{2}\right\}
$$

where

$$
r=\sqrt{\left(\frac{r_{x}-c_{x} \tau}{L_{x}}\right)^{2}+\left(\frac{r_{y}-c_{y} \tau}{L_{y}}\right)^{2}}
$$

and $a=3.337$. The parameters $L_{x}, L_{y}, T, c_{x}$, and $c_{y}$ are spatially varying. They are not determined a priori, but rather estimated by fitting (5.51) to the observed SSH correlation function during the AVISO data mapping (Le Traon, 2012, personal communication). Brachet et al. [2004] compare the observed correlation function with (5.51), and map the parameters used for the North Atlantic.

As discussed in Ch. 2 this covariance function results in a gridded altimetry product that falls off too quickly at high frequency and wavenumber, relative to the 
along-track altimetry and other similar observations. This means that the covariance function used is smoothing some of the small period and wavelength features present in the along-track altimetry. An alternative would be to use the covariance function (5.35) based on (5.21). To the best of our knowledge, the origin and accuracy of (5.51) are not discussed in the literature. In contrast, the model spectrum (5.21) is constructed specifically to fit a range of ocean observations, including near surface currents, current meter observations, and the along-track altimetry itself.

Figure 5-12 shows the correlation function (5.51), calculated using parameters from Brachet et al. [2004] at $30^{\circ} \mathrm{N}, 320^{\circ} \mathrm{E}$ in the North Atlantic, compared with the correlation function based on the model spectrum (5.21) at the same location. The two correlation functions have similar elliptical shape and westward drift with increasing temporal lag. However, the model correlation function has a larger correlation wavelength.

Figure 5-13 shows another comparison of the AVISO and model correlation functions for SSH, as a function of zonal and temporal lag. The AVISO correlation function approaches zero by $150 \mathrm{~km}$ lag, while the model correlation function still has a small correlation at $200 \mathrm{~km}$. The shapes of the two correlation functions also differ. In the AVISO function, correlation is higher for westward motions at all frequencies, while the model spectrum becomes isotropic at very small scales.

\subsection{Conclusions}

This chapter presents a model for the spectrum of low-frequency ocean variability. The model is presented in terms of a four-dimensional frequency-wavenumber spectrum for the streamfunction. The model has a universal functional form but geographically varying parameters. We also present a set of characteristic functions relating the streamfunction spectrum to the spectrum of other variables.

The model presented here describes ocean variability with periods from about 10 days to 15 years, and wavelengths of hundreds to thousands of kilometers. It is meant as a low-frequency analogue of the internal wave spectrum described by Garrett and 

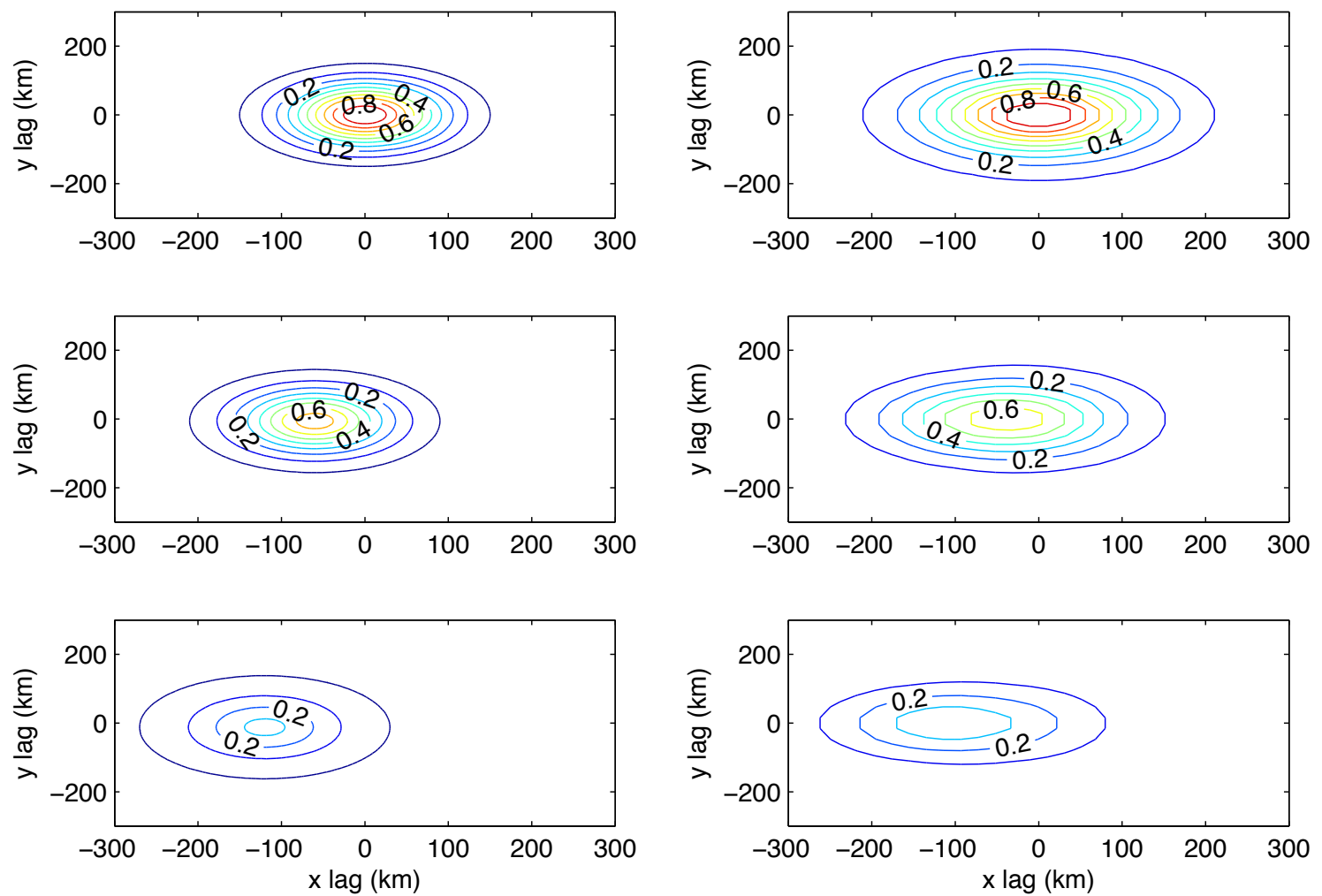

Figure 5-12: Spatial correlation function for SSH used in the AVISO data mapping (left) and based on the model spectrum (5.21) (right) at lags of 0 days (top), 20 days (middle), and 40 days (bottom) at $30^{\circ} \mathrm{N}, 320^{\circ} \mathrm{E}$ in the North Atlantic. Contour interval is 0.1 .
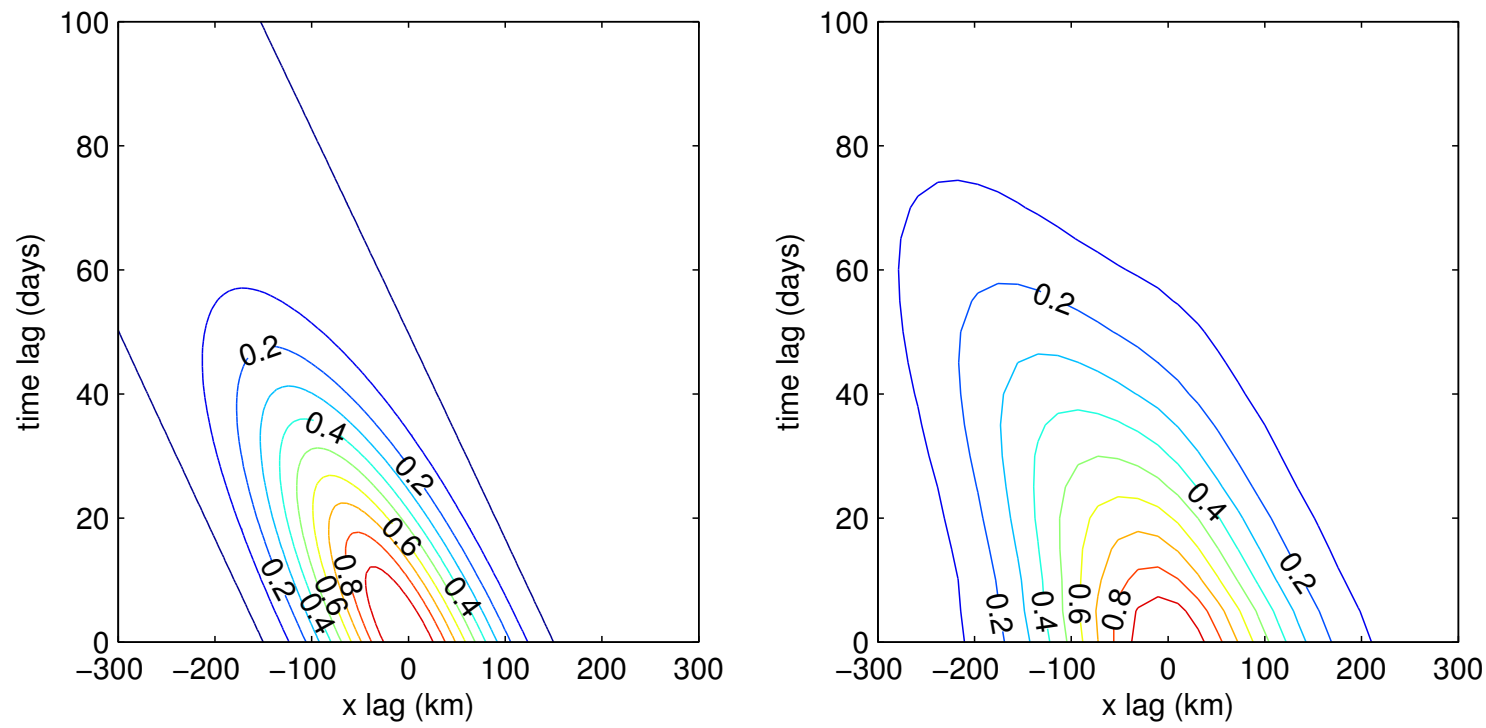

Figure 5-13: Zonal-temporal correlation function for SSH used in the AVISO data mapping (left) and based on the model spectrum (5.21) (right) at $30^{\circ} \mathrm{N}, 320^{\circ} \mathrm{E}$ in the North Atlantic. Contour interval is 0.1. 
Munk [1972, 1975]. As with the Garrett and Munk spectrum, the model is empirical, guided by the following measurements: SSH from satellite altimetry, near-surface currents from shipboard ADCP, velocity measurements from moored current meters, and moored temperature records.

The model presented here is intended to be consistent with observed spectra on average. However, many regions of the ocean have peculiar dynamics and the model does not fit all regions. In particular, the model is not intended to match observations in the core of western boundary currents, in the ACC, within about $5^{\circ}$ of the equator, or poleward of $50^{\circ}$.

This chapter is an update to the model presented by Zang [2000] and Zang and Wunsch [2001]. The main respect in which the model described here differs from earlier versions is that we have relaxed the assumptions of isotropy and homogeneity. Dominant periods, wavelengths, phase speeds, and amplitude vary geographically, as seen from altimetry and other observations. Also, the model is anisotropic; westward motions dominate.

Our empirical model of the streamfunction spectrum is clearly quite contrived, and is intended to describe the observations, rather than to lay out the underlying dynamics. In its functional form, (5.21) is constructed so as to be quite malleable. The parameters $L_{x}, L_{y}, T, c_{x}, c_{y}, d_{1}$, and $d_{2}$ controlling dominant wavelengths, periods, and phase speeds, and the parameter $n$ controlling the high-wavenumber spectral slope can all be tuned to match the observations. This functional form is not unique, and others certainly exist that would do as well or better.

Non-uniqueness notwithstanding, our model fulfills some apparent requirements based on the observations. Major requirements include the dominance of westward phase speed and the transition from an approximate power law at high frequency and wavenumber to a plateau at low frequency and wavenumber. A more subtle point concerns the relationship between moored frequency spectra for temperature an energy. As noted in $§ 5.3 .4$, moored frequency spectra for temperature and kinetic energy both appear to approach an $\omega^{-2}$ power law at high frequency. Our model is consistent with this observation. We have considered many alternatives to the power 
law term in (5.21), and none produced as good agreement with the range of observed spectra.

The Gaussian term in (5.21) introduces anisotropy into the model spectrum. The Gaussian function was chosen out of convenience, and because it produces a qualitative match to the observed SSH spectrum. However, the observed SSH spectrum considered here is forced to have a Gaussian shape by the covariance function (5.51) used in the AVISO data mapping. This aspect of the model spectrum should be re-evaluated using un-mapped altimetric observations.

The model is based on a number of assumptions, all of which are likely to be violated to some extent.

a) Observed motions are assumed to be the result of a linear superposition of waves with random phase. In particular, the presence of nonlinear coherent vortices is neglected [Chelton et al., 2011].

b) Temperature variability is assumed to be due primarily to vertical advection of the mean temperature gradient, and not to horizontal advection. The failure of this assumption is one of the likely causes of the poor fit in Fig. 5-8.

c) The uncertainty estimates based on the model spectrum assume that the observations represent a Gaussian random field. But SSH variability is apparently non-Gaussian [Sura and Gille, 2010]. A few regions, including the Gulf Stream, Kuroshio, and Agulhas Retroflection displayed bimodal patterns of SSH variability. Elsewhere, variability was unimodal, skewness is close to zero, and kurtosis is close to 3 (as for Gaussian variability). The statistics of the measurements should be taken into account when applying the model spectrum.

d) The present model assumes a universal high-wavenumber spectral slope. As discussed above, the actual spectral slope is the subject of active debate. As more reliable estimates of the spectral slopes become available this model could be updated.

The primary motivation for this project is to provide a description of the periods 
and wavelengths of ocean variability that will be useful to further studies. To that end, we have described a few applications for the model spectrum. First, we use the model spectrum to estimate spatial and temporal correlation functions for temperature and velocity. Second, we use the model spectrum and associated covariance functions to estimate the uncertainty in a trend in ocean temperature. This application can easily be extended to apply to other quantities. The question can also be inverted to aid in experiment design. Instead of asking "What is the uncertainty resulting from a given measurement?" we could ask "How many measurements do we need to make to achieve the desired uncertainty?" Finally, we suggest that the model spectrum can be used to improve gridded altimetric data products or the assimilation of altimetry into numerical models.

We hope that others will find more applications for this model, and so briefly summarize the calculation of the spectrum. The functional form for the horizontal wavenumber-frequency spectrum is given by (5.21). The geographically variable dominant periods and wavelengths are given by (5.27)-(5.29). For the zonal phase speed, we use the long Rossby wave phase speed for a rough-bottom ocean, as calculated in Ch. 4. In practice, reasonable approximation would be to use the flat-bottom long Rossby wave phase speed, which can easily be calculated using the deformation radius tabulated by Chelton et al. [1998], or to use phase speeds estimated by Jacobs et al. [2001] or Fu [2009].

The vertical structure is described in terms of the modes for linear Rossby waves over rough topography, as described in Ch. 4. These modes depend on climatological temperature and salinity profiles and on the bottom roughness.

The amplitude of the model spectrum is set based on observed surface eddy kinetic energy. This is easily calculated directly from available altimetric data. Alternatively Zang and Wunsch [2001] provide an approximate empirical formula for the surface eddy kinetic energy.

Together, the horizontal wavenumber-frequency spectrum, vertical structure, and amplitude describe the four-dimensional spectrum of the streamfunction. The spectrum for other variables can be calculated using the appropriate characteristic func- 
tion. 


\section{Chapter 6}

\section{Conclusions}

This thesis is an attempt to provide a summary of the space and time scales of lowfrequency ocean variability. No process can be fully understood without the broader context of other scales of ever-present ocean variability. In particular, knowledge of the dominant scale of variability, and length over which observations are correlated, is important for observing system design and for determining the significance of apparent trends.

This effort is largely descriptive of a generic ocean region. An average description is valuable both because it is found to apply to much of the ocean, and also because it can help to identify abnormal regions and the unique dynamical processes at work. There are many aspects of the model presented that defy explanation. In this sense, the model is a challenge to observationalists to disprove it and to theorists to explain it.

In this thesis, we present an updated empirical model for the four-dimensional frequency-wavenumber spectrum of low-frequency ocean variability. This model serves as a major update to the model of Zang [2000], Zang and Wunsch [2001], and Wunsch [2010] by proposing a non-separable form and including the geographical variation in spectral shape seen in observations. We also revise the description of the vertical structure of variability. 


\subsection{Summary}

The model is based in part on 19 years of gridded altimetric data. In Ch. 2, we describe the three-dimensional frequency-wavenumber spectrum of sea surface height. The most striking feature of the spectrum is the nearly ubiquitous presence of a ridge of westward variance. This ridge is straight in frequency-wavenumber space, representing nondispersive motions. As noted by earlier authors, the dominant motions propagate with a westward phase speed significantly faster than the classical Rossby wave theory predicts. In contrast to the zonal propagation, there is no dominant phase speed for meridional propagation. Instead, a wide range of meridional wavelengths and phase speeds are observed.

Dominant periods and wavelengths, estimated as the inverse of the first moment of the spectrum, also vary geographically. Periods are shortest near the equator, at less than 100 days, increasing to 300-400 days at high latitude. Wavelengths decrease with latitude, from about $1500 \mathrm{~km}$ near the equator to $500 \mathrm{~km}$ at high latitude.

One of the contributions of this chapter is to illustrate the value of the threedimensional spectrum. It shows, for example, the signature of zonal velocity striations at low frequency and explains the difference between along- and across-track velocity spectra. However, the ease of computing the three-dimensional spectrum from gridded altimetry comes at a cost. The objective mapping procedure used to produce the gridded data product significantly distorts the SSH spectrum, especially at high frequency and wavenumber. In retrospect, we would not use the gridded altimetry product for such a study.

In Ch. 3 we explore the vertical structure kinetic energy using moored current meters and the ECCO2 GCM. The main result of this chapter is that the vertical structure of kinetic energy in the ECCO2 model is inconsistent with the classic theory of free, linear Rossby waves in a flat bottom, resting ocean. In particular, decomposing vertical profiles of horizontal velocity into the classic Rossby wave modes results in strong coupling between the barotropic and first baroclinic modes. EOF analysis suggests that appropriate vertical mode shape is similar to the classic first baroclinic 
mode, but shifted such that it approaches zero at the ocean bottom. This vertical structure implies weaker abyssal kinetic energy than would exist in the absence of coupling. This apparent coupling is nearly global, and must be accounted for in the vertical structure of the model spectrum.

Uncertainty about the accuracy of the ECCO2 model mean that the conclusions of this chapter must be taken as provisional. We hope to be able to duplicate these results with current meter records. However, existing current meter records are not sufficient to confirm or reject the mode coupling observation. The main barriers are insufficient record length and poor vertical resolution. We considered one pair of moorings in particular. The moorings were deployed in the same location in succeeding years. During the first year, the barotropic and baroclinic modes calculated from the mooring showed strong coupling consistent with the ECCO2 model findings. In the second year, the modes were completely incoherent.

In Ch. 4 we discuss various theoretical explanations for the observed spectrum. Two features are of particular interest: the too-fast propagation speed, and the coupled mode vertical structure. We show that free, linear Rossby waves over a roughbottom exhibit both of these features. Rough topography is imposed through the bottom boundary condition. We solve the QG potential vorticity equation numerically with climatological stratification and realistic topography. Rough topography alters the vertical structure of the Rossby wave modes, resulting in a mode shape that is consistent with that seen in the ECCO2 model. Rough topography also increases the zonal phase speed of Rossby waves. The phase speed enhancement depends on both stratification and on the amplitude of the topography. Predicted phase speeds are in good agreement with phase speeds estimated from altimetry. We find that the sea floor is sufficiently rough nearly everywhere to impact the speed of Rossby waves. Hence, rough-topography is a possible explanation for the observed mode coupling seen in the ECCO2 model.

Several other theories have been advanced for explaining the observed phase speed and vertical structure of low-frequency variability, and are likely to play a role. Mean flow and rough topography can combine to increase the phase speed of long 
Rossby waves and create a vertical structure very similar to observations [Tailleux and McWilliams, 2001; Killworth and Blundell, 2005; Aoki et al., 2009]. It has also been suggested that the SSH observations are dominated by nonlinear coherent vortices, rather than linear waves [Chelton et al., 2011]. Nonlinear coherent vortices have a phase speed and dispersion relation similar to observations [Early et al., 2011]. Furthermore, coherent vortices have equivalent barotropic vertical structure, again similar to observations [McWilliams and Flierl, 1979]. Finally, surface-forced modes due to either atmospheric forcing [Müller and Frankignoul, 1981] or surface buoyancy anomalies [Lapeyre and Klein, 2006] can also result in surface-intensified vertical structure.

These theories all predict qualitatively similar vertical structure, making difficult the task of distinguishing between them. This task deserves further attention. Here, we have focused on the linear theory with rough topography since it seems to be the simplest theory that accounts for the coupled-mode vertical structure. However, the apparent nonlinearity discussed in Ch. 2 strongly suggests the coherent vortices play a role.

In Ch. 5 we present an updated model of the frequency-wavenumber spectrum of low-frequency oceanic variability. The model has a universal functional form, but geographically varying parameters. The most important revision to the model presented by Zang [2000] is the inclusion of geographically varying parameters that control the dominant periods and wavelengths of the spectrum. The universal spectrum of Zang [2000] could not account for the meridional changes in dominant period and wavelength.

The model spectrum is composed of two terms. The first is a simple power law, isotropic in wavenumber, intended to describe the high frequency and wavenumber limits of the spectrum. This model accounts for the anisotropy in the SSH spectrum seen in Ch. 2 by adding a Gaussian term with defined zonal and meridional phase speed.

We discuss four applications for the model spectrum. The most straightforward application is the estimation of unobserved spectra. Zang [2000] provided a set of 
characteristic functions, based on linear geostrophic dynamics, that give the spectrum for many variables in terms of the model streamfunction spectrum. Second, the model spectrum can be used to estimate the space and time correlation function for any variable. Third, we show how the model can be used to estimate the uncertainty in a mean or trend in oceanic quantities such as temperature or heat content. Such estimates can also inform observing system design. Finally, we suggest that the model spectrum can be used to improve gridded altimetric data products or the assimilation of altimetry into numerical models. Objective mapping of the altimetric data relies on an imposed covariance function. Currently, the gridded altimetric data product is heavily smoothed at small period and wavelength. A more accurate covariance function would improve the accuracy of the gridded data product.

While the present model is certainly not the final word on the periods and wavelengths of ocean variability, we hope that it has reached a state where it can be usefully applied. The most immediate applications are in observing system design, experiment planning, data analysis and interpretation, and numerical model evaluation. We also hope that the model presented here can help to focus observations. In particular, the vertical structure of variability is very uncertain. We propose a horizontal wavenumber-frequency spectrum that is independent of depth. Can observations be made to confirm or reject this hypothesis? Our model proposes a universal spectral slope for the high-wavenumber and high-frequency limit, though the wavenumber slope appears to be steeper in high kinetic energy regions and flatter in low kinetic energy regions and near the equator. Is this change related to different turbulent regimes, or the rate of baroclinic instability, or some other cause?

By making a concrete statement about the spectrum of low-frequency oceanic variability, we pose a challenge to theoreticians to explain it. What determines the kinetic energy level, which is seen to vary by over four orders of magnitude across the ocean? And what sets the wavenumber spectral slope? Many theories have been proposed, but none can explain the observations. 


\subsection{Future directions}

Aside from incorporating more extensive observations, there are a number of ways that the present model could be improved. We list a number of assumptions in the conclusion of Ch. 5. Each of these should be evaluated. Also, the present model focuses on matching observed phase speeds, since these are more easily estimated from altimetry. However, energy travels at the group velocity, and so this is a more dynamically interesting quantity. Can group velocity be determined from observations? If so, how does it compare to the model spectrum?

The model spectrum presented here only attempts to describe frequencies between about 10 days and 15 years. However, the spectrum of "background noise" at low frequency has a significant impact on our ability to distinguish true secular trends from apparent trends arising from low frequency variability [Wunsch, 2008]. In particular, a "red noise" spectrum implies strong correlation over very long periods, which can appear as a secular trend even when none is present. The extension of this model to lower frequencies is an important task for the future.

Perhaps the most important area open for improvement is in the description of the vertical structure. We suggest that dedicated numerical modeling studies be run to determine the relative importance of mean flow, rough topography, surface forcing, and nonlinearity in setting the vertical structure of variability.

A second major area for improvement is the inclusion of coherent structures. In adopting a spectral description, we implicitly assume that the low-frequency variability is describable as a superposition of independent wave components. However, coherent structures appear to be responsible for a significant fraction of SSH variance [Chelton et al., 2011] and cannot be accommodated by linear wave description.

Third, the parameters $L_{x}, L_{y}, T$, and $n$ in the model (5.21) should be allowed to vary in latitude and longitude. In principle, this is easy, but requires an algorithm for estimating the parameters from observations.

Finally, there is the important and fascinating task of explaining the observed variability. How much of the variability is forced directly by the atmosphere, and how 
much is generated through baroclinic instability? To what extent are the observed spectral slopes due to turbulent energy cascades? What sets the dominant period and wavelength, and why do they vary with latitude? Ch. 4 considers these questions superficially, but there is much more work to be done. 


\section{Appendix A}

\section{Numerical methods}

The results of $\S 4.2$ are based on numerical solutions of the linearized QGPV equation, with topography included in the bottom boundary condition:

$$
\begin{gathered}
\frac{\partial^{2} P}{\partial y^{2}}+\frac{\partial}{\partial z}\left(\frac{f_{0}^{2}}{N^{2}} \frac{\partial P}{\partial z}\right)-\left(4 \pi^{2} k^{2}+\frac{k \beta}{\omega}\right) P=0 \\
\frac{\partial P}{\partial z}=0 \quad \text { at } \quad z=0 \\
\frac{\partial P}{\partial z}=\frac{N^{2} k}{f_{0} \omega} h_{y} P \quad \text { at } \quad z=-H
\end{gathered}
$$

(See $\S 4.2$ for a derivation of the equations and definitions of the varibales.) Equations A.1 form a 2-dimensional eigenvalue problem on a rectangular domain $0 \leq y \leq$ $L,-H \leq z \leq 0$, where the eigenvector $P(y, z)$ is the meridional-vertical part of the pressure variation and the eigenvalue $\omega$ is the frequency of wave propagation. $\mathrm{Nu}-$ merical solutions are obtained by a simple matrix method using the grid shown in Fig. A-1. 


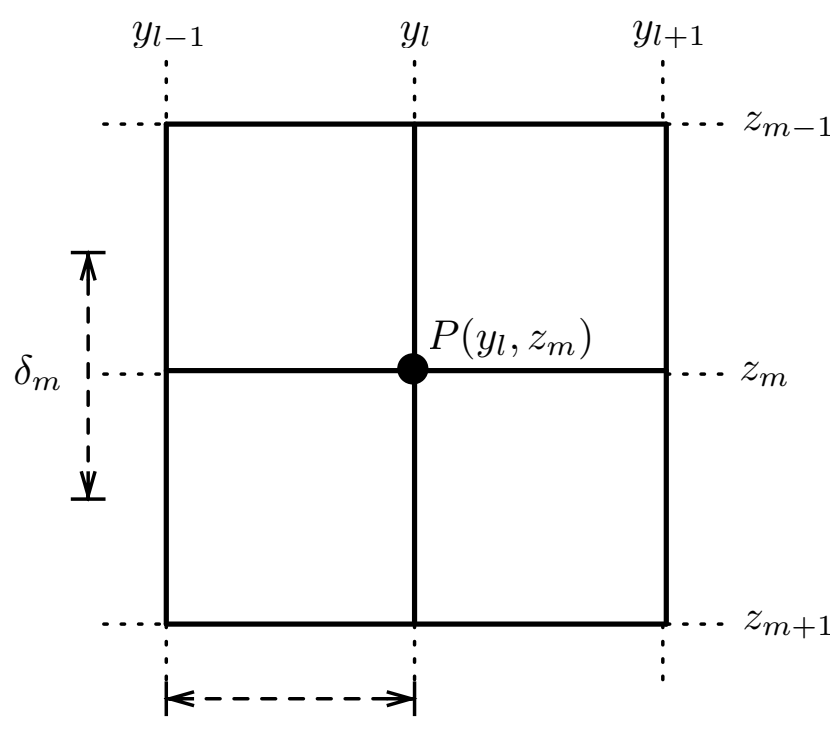

$\Lambda$

Figure A-1: The grid used to discretize (A.1).

Horizontal derivative The first term in (A.1) is approximated by its second centered difference

$$
\frac{\partial^{2} P\left(y_{l}, z_{m}\right)}{\partial y^{2}}= \begin{cases}\frac{P\left(y_{2}, z_{m}\right)-2 P\left(y_{1}, z_{m}\right)+P\left(y_{L-1}, z_{m}\right)}{\Lambda^{2}} & l=1 \\ \frac{P\left(y_{l+1}, z_{m}\right)-2 P\left(y_{l}, z_{m}\right)+P\left(y_{l-1}, z_{m}\right)}{\Lambda^{2}} & l=2 \ldots L-1 \\ \frac{P\left(y_{2}, z_{m}\right)-2 P\left(y_{L}, z_{m}\right)+P\left(y_{L-1}, z_{m}\right)}{\Lambda^{2}} & l=L\end{cases}
$$

for $l=1,2, \ldots L$ and $m=1,2, \ldots M$, where $\Lambda=y_{l+1}-y_{l}$ is the horizontal separation between grid points. The boundary conditions require $P$ and its $y$ derivative to be periodic in $y$.

Vertical derivative The second term in (A.1) involves the vertical derivative of the buoyancy frequency $N(z)$. Chelton et al. [1998] compared several discrete approximations to the buoyancy frequency profile and recommended a neutral density gradient method based on centered first differences of the potential density referenced to the midpoint between two depths. In this method, the buoyancy frequency squared 
is approximated as

$$
N^{2}\left(y_{l}, z_{m+1 / 2}\right)=-\frac{g}{\rho_{0}}\left(\frac{\rho\left(y_{l}, z_{m} \rightarrow z_{m+1 / 2}\right)-\rho\left(y_{l}, z_{m+1} \rightarrow z_{m+1 / 2}\right)}{z_{m}-z_{m+1}}\right)
$$

where $z_{m+1 / 2} \equiv\left(z_{m}+z_{m+1}\right) / 2$ is the midpoint between two model grid points and the notation $\rho\left(y_{l}, z_{m} \rightarrow z_{m+1 / 2}\right)$ is shorthand for the potential density of a water parcel at depth $z_{m}$ referenced to $z_{m+1 / 2}$. The finite difference form of the second term in (A.1) becomes

$$
\frac{\partial}{\partial z}\left(\frac{f_{0}^{2}}{N^{2}} \frac{\partial P\left(y_{l}, z_{m}\right)}{\partial z}\right)=\frac{f_{0}^{2} \rho_{0}}{g} \begin{cases}\frac{2}{\delta_{1}}\left(\frac{P\left(y_{l}, z_{2}\right)-P\left(y_{l}, z_{1}\right)}{\rho\left(y_{l}, z_{2}\right)-\rho\left(y_{l}, z_{1}\right)}\right) & m=1 \\ \frac{1}{\delta_{m}}\left(\frac{P\left(y_{l}, z_{m-1}\right)-P\left(y_{l}, z_{m}\right)}{\rho\left(y_{l}, z_{m}\right)-\rho\left(y_{l}, z_{m-1}\right)}\right. & m=2 \ldots M-1 \\ \left.-\frac{P\left(y_{l}, z_{m}\right)-P\left(y_{l}, z_{m+1}\right)}{\rho\left(y_{l}, z_{m+1}\right)-\rho\left(y_{l}, z_{m}\right)}\right) & \\ \frac{2}{\delta_{M}}\left(\frac{P\left(y_{l}, z_{M-1}\right)-(1+\Delta) P\left(y_{l}, z_{M}\right)}{\rho\left(y_{l}, z_{M}\right)-\rho\left(y_{l}, z_{M-1}\right)}\right) & m=M\end{cases}
$$

where $\delta_{m}=\left(z_{m-1}-z_{m+1}\right) / 2$ is the distance between midpoints of the model grid and

$$
\Delta=\frac{k g}{f_{0} \rho_{0} \omega}\left(\rho\left(y_{l}, z_{M}\right)-\rho\left(y_{l}, z_{M-1}\right)\right)\left(\frac{h\left(y_{l+1}, z_{M}\right)-h\left(y_{l}, z_{M}\right)}{\Lambda}\right)
$$

represents the effect of topography in the bottom boundary condition.

Linear indexing The two-dimensional discrete domain used above is intractable with a simple matrix approach, and so we switch from array to linear indexing, as described in Press et al. $[2007, \S 20.0 .2]$. Introducing a new linear index $i=(l-1) M+$ $m$, we have the new notation $P_{i}=P\left(y_{l}, z_{m}\right)$ and, for example, $P_{i+M}=P\left(y_{l+1}, z_{m}\right)$. Using the new index and re-grouping terms, the discrete form of (A.1) may be written as

$$
\omega\left(A_{i j}+B_{i j}+C_{i j}\right) P_{j}+D_{i j} P_{j}=0
$$


with summation over the repeated index implied. The matrix operators are

$$
\begin{aligned}
& A_{i j} P_{j}= \begin{cases}\frac{P_{i+M}-2 P_{i}+P_{i+(L-2) M}}{\Lambda^{2}} & i=1,2, \ldots M \\
\frac{P_{i-(L-2) M}-2 P_{i}+P_{i-M}}{\Lambda^{2}} & i=(L-1) M+1,(L-1) M+2, \ldots(L-1) M+M \\
\frac{P_{i+M}-2 P_{i}+P_{i-M}}{\Lambda^{2}} & \text { otherwise }\end{cases} \\
& B_{i j} P_{j}=\frac{f_{0}^{2} \rho_{0}}{g} \begin{cases}\frac{2}{\delta_{i}}\left(\frac{P_{i+1}-P_{i}}{\rho_{i+1}-\rho_{i}}\right) & i=1, M+1, \ldots(L-1) M+1 \\
\frac{2}{\delta_{i}}\left(\frac{P_{i-1}-P_{i}}{\rho_{i}-\rho_{i-1}}\right) & i=M, 2 M, \ldots L M \\
\frac{1}{\delta_{i}}\left(\frac{P_{i-1}-P_{i}}{\rho_{i}-\rho_{i-1}}-\frac{P_{i}-P_{i+1}}{\rho_{i+1}-\rho_{i}}\right) & \text { otherwise }\end{cases} \\
& C_{i j} P_{j}=-4 \pi^{2} k^{2} P_{j} \quad i=1,2, \ldots L M \\
& D_{i j} P_{j}= \begin{cases}-k \beta P_{i}-\frac{2 f_{0} k}{\delta_{i}}\left(\frac{h_{i+M}-h_{i}}{\Lambda}\right) P_{i} & i=M, 2 M, \ldots L M \\
-k \beta P_{i} & \text { otherwise }\end{cases}
\end{aligned}
$$

The matrix eigenvalue problem (A.6) is solved numerically and the resulting eigenvectors $P_{i}$ are transformed back to the physical domain.

Solution by separation of variables Equation (A.1a) is separable in $y$ and $z$, allowing an alternative method of solving the problem, though we have not attempted it. Assuming solutions of the form $P(y, z)=Y(y) Z(z)$, we find

$$
\frac{1}{Y} \frac{\mathrm{d}^{2} Y}{\mathrm{~d} y^{2}}-\left(4 \pi^{2} k^{2}+\frac{k \beta}{\omega}\right)=-\frac{1}{Z} \frac{\mathrm{d}}{\mathrm{d} z}\left(\frac{f_{0}^{2}}{N^{2}} \frac{\mathrm{d} Z}{\mathrm{~d} z}\right)=\Gamma
$$

Numerical solutions could be sought by solving two one-dimensional eigenvalue problems, rather than the single two-dimensional problem described above.

While the interior equation is separable, the bottom boundary condition is not 
separable with arbitrary topography. Using $P(y, z)=Y(y) Z(z)$ in (A.1c), we have

$$
\frac{1}{Z} \frac{\partial Z}{\partial z}=\frac{N^{2} k}{f_{0} \omega} h_{y}
$$

The only separable solution requires $h_{y}=$ constant. 


\section{Bibliography}

Adcroft, A., J.-M. Campin, C. Hill, and J. Marshall, Implementation of an atmosphere-ocean general circulation model on the expanded spherical cube, Monthly Weather Review, 132(12), 2845-2863, 2004.

Aoki, K., A. Kubokawa, H. Sasaki, and Y. Sasai, Midlatitude baroclinic Rossby waves in a high-resolution OGCM simulation, Journal of Physical Oceanography, 39(9), 2264-2279, 2009.

Arbic, B. K., and G. R. Flierl, Coherent vortices and kinetic energy ribbons in asymptotic, quasi two-dimensional f-plane turbulence, Physics of Fluids, 15(8), 2177-2189, 2003.

Arbic, B. K., and G. R. Flierl, Effects of mean flow direction on energy, isotropy, and coherence of baroclinically unstable beta-plane geostrophic turbulence, Journal of Physical Oceanography, 34 (1), 77-93, 2004a.

Arbic, B. K., and G. R. Flierl, Baroclinically unstable geostrophic turbulence in the limits of strong and weak bottom Ekman friction: Application to midocean eddies, Journal of Physical Oceanography, 34(10), 2257-2273, 2004b.

Arbic, B. K., R. B. Scott, G. R. Flierl, A. J. Morten, J. G. Richman, and J. F. Shriver, Nonlinear cascades of surface oceanic geostrophic kinetic energy in the frequency domain, Journal of Physical Oceanography in press, 2012.

AVISO, SSALTO/DUACS user handbook: (M)SLA and (M)ADT near-real time and delayed time products, Tech. Rep. CLS-DOS-NT-06-034, CNES, 2012.

Baehr, J., K. Keller, and J. Marotzke, Detecting potential changes in the meridional overturning circulation at $26^{\circ} \mathrm{N}$ in the Atlantic, Climate Change, 91(1-2), 11-27, 2008a.

Baehr, J., D. McInerney, K. Keller, and J. Marotzke, Optimization of an observing system design for the North Atlantic meridional overturning circulation, Journal of Atmospheric and Oceanic Technology, 25(4), 625-634, 2008b.

Barth, N. H., Oceanographic experiment design II: Genetic algorithms, Journal of Atmospheric and Oceanic Technology, 9(4), 434-443, 1992. 
Barth, N. H., and C. Wunsch, Oceanic experimental design by simulated annealing, Journal of Physical Oceanography, 20(9), 1249-1263, 1990.

Bernstein, R. L., and W. B. White, Zonal variability in the distribution of eddy energy in the mid-latitude North Pacific Ocean, Journal of Physical Oceanography, 7(1), 123-126, doi:10.1175/1520-0485(1977)007<0123:ZVITDO>2.0.CO;2, 1977.

Blumen, W., Uniform potential vorticity flow: Part I. theory of wave interactions and two-dimensional turbulence, Journal of the Atmospheric Sciences, 35 (5), 774-783, doi:10.1175/1520-0469(1978)035<0774:UPVFPI>2.0.CO;2, 1978.

Bobrovich, A. V., and G. M. Reznik, Planetary waves in a stratified ocean of variable depth. Part 2. continuously stratified ocean, Journal of Fluid Mechanics, 388, 147$169,1999$.

Brachet, S., P.-Y. Le Traon, and C. Le Provost, Mesoscale variability from a highresolution model and from altimeter data in the North Atlantic Ocean, Journal of Geophysical Research, 109, C12,025, doi:10.1029/2004JC002360, 2004.

Bretherton, F., R. Davis, and C. Fandry, A technique for objective analysis and design of oceanographic experiments applied to MODE-73, Deep Sea Research and Oceanographic Abstracts, 23(7), 559-582, doi:10.1016/0011-7471(76)90001-2, 1976.

Challenor, P. G., P. Cipollini, and D. Cromwell, Use of the 3D Radon transform to examine the properties of oceanic Rossby waves, Journal of Atmospheric and Oceanic Technology, 18(9), 1558-1566, 2001.

Charney, J. G., Geostrophic turbulence, Journal of the Atmospheric Sciences, 28, 1087-1097, 1971.

Chatfield, C., The Analysis of Time Series: An Introduction, sixth ed., Chapman \& Hall/CRC, 2004.

Chelton, D. B., and M. G. Schlax, Global observations of oceanic Rossby waves, Science, 272(5259), 234-238, 1996.

Chelton, D. B., R. A. de Szoeke, M. G. Schlax, K. El Naggar, and N. Siwertz, Geographical variability of the first baroclinic Rossby radius of deformation, Journal of Physical Oceanography, 28(3), 433-460, 1998.

Chelton, D. B., F. J. Wentz, C. L. Gentemann, R. A. de Szoeke, and M. G. Schlax, Satellite microwave SST observations of transequatorial tropical instability waves, Geophysical Research Letters, 27(9), 1239-1242, 2000.

Chelton, D. B., M. G. Schlax, R. M. Samelson, and R. A. de Szoeke, Global observations of large oceanic eddies, Geophysical Research Letters, 34, L15,606, 2007.

Chelton, D. B., M. G. Schlax, and R. M. Samelson, Global observations of nonlinear mesoscale eddies, Progress in Oceanography, 91(2), 167-216, doi: 10.1016/j.pocean.2011.01.002, 2011. 
Cummings, J. A., Operational multivariate ocean data assimilation, Quarterly Journal of the Royal Meteorological Society, 131(613), 3583-3604, doi: 10.1256/qj.05.105, 2005.

de Szoeke, R. A., and D. B. Chelton, The modification of long planetary waves by homogeneous potential vorticity layers, Journal of Physical Oceanography, 29(3), 500-511, 1999.

Dewar, W. K., On "too fast" baroclinic planetary waves in the general circulation, Journal of Physical Oceanography, 28(9), 1739-1758, 1998.

Ducet, N., P.-Y. Le Traon, and G. Reverdin, Global high-resolution mapping of ocean circulation from TOPEX/POSEIDON and ERS-1 and -2, Journal of Geophysical Research, 105(C8), 19,477-19,498, 2000.

Durland, T. S., R. M. Samelson, D. B. Chelton, and R. A. de Szoeke, Modification of long equatorial Rossby wave phase speeds by zonal currents, Journal of Physical Oceanography, 41(6), 1077-1101, doi:10.1175/2011JPO4503.1, 2011.

Early, J. J., R. M. Samelson, and D. B. Chelton, The evolution and propagation of quasigeostrophic ocean eddies, Journal of Physical Oceanography, 41(8), 1535$1555,2011$.

Farrar, J. T., Observations of the dispersion characteristics and meridional sea level structure of equatorial waves in the Pacific Ocean, Journal of Physical Oceanography, 38(8), 1669-1689, 2008.

Farrar, J. T., and R. A. Weller, Intraseasonal variability near $10^{\circ} \mathrm{N}$ in the eastern tropical Pacific Ocean, Journal of Geophysical Research, 111, C05,015, 2006.

Ferrari, R., and M. Nikurashin, Suppression of eddy diffusivity across jets in the Southern Ocean, Journal of Physical Oceanography, 40(7), 1501-1519, 2010.

Ferrari, R., and C. Wunsch, The distribution of eddy kinetic and potential energies in the global ocean, Tellus, 62(2), 92-108, tellus, 2010.

Forget, G., Mapping ocean observations in a dynamical framework: a 2004-2006 ocean atlas, Journal of Physical Oceanography, 40(6), 1201-1221, 2010.

Forget, G., and C. Wunsch, Estimated global hydrographic variability, Journal of Physical Oceanography, 37(8), 1997-2008, doi:10.1175/JPO3072.1, 2007.

Frankignoul, C., and P. Müller, Quasi-geostrophic response of an infinite -plane ocean to stochastic forcing by the atmosphere, Journal of Physical Oceanography, 9(1), 104-127, 1979a.

Frankignoul, C., and P. Müller, On the generation of geostrophic eddies by surface buoyancy flux anomalies, Journal of Physical Oceanography, 9(6), 1207-1213, 1979b. 
Fu, L.-L., Pattern and velocity of propagation of the global ocean eddy variability, Journal of Geophysical Research, 114, C11,017, 2009.

Fu, L.-L., and A. Cazenave (Eds.), Satellite altemitery and earth sciences: a handbook of techniques and applications, International Geophysics Series, vol. 69, Academic Press, San Diego, CA, 2001.

Fu, L.-L., E. J. Christensen, C. A. Yamarone Jr., M. Lefebvref, Y. Ménard, M. Dorrer, and P. Escudier, Topex/poseidon mission overview, Journal of Geophysical Research, 99(C12), 14,369-24,381, 1994.

Fu, L.-L., D. B. Chelton, P.-Y. Le Traon, and M. Rosemary, Eddy dynamics from satellite altimetry, Oceanography, 23(4), 14-25, 2010.

Garrett, C., and W. Munk, Space-time scales of internal waves, Geophysical Fluid Dynamics, 2, 225-264, 1972.

Garrett, C., and W. Munk, Space-time scales of internal waves: a progress report, Journal of Geophysical Research, 80(3), 291-297, 1975.

Gill, A. E., Atmosphere-Ocean Dynamics, International Geophysics Series, vol. 30, Academic Press, San Diego, 1982.

Gille, S. T., Float observations of the Southern Ocean. Part II: Eddy fluxes, Journal of Physical Oceanography, 33(6), 1182-1196, 2003.

Glazman, R. E., and P. B. Weichman, Meridional component of oceanic Rossby wave propagation, Dynamics of Atmospheres and Oceans, 38, 173-193, 2005.

Heimbach, P., C. Wunsch, R. M. Ponte, G. Forget, C. Hill, and J. Utke, Timescales and regions of the sensitivity of atlantic meridional volume and heat transport: Toward observing system design, Deep Sea Research Part II: Topical Studies in Oceanography, 58(11-17), 1858-1879, 2011.

Held, I. M., R. T. Pierrehumbert, S. T. Garner, and K. L. Swanson, Surface quasigeostrophic dynamics, Journal of Fluid Mechanics, 282, 1-20, 1995.

Hill, K. L., I. S. Robinson, and P. Cipollini, Propagation characteristics of extratropical planetary waves observed in the ATSR global sea surface temperature record, Journal of Geophysical Research, 105 (C9), 21,927-21,945, 2000.

Hirschi, J., J. Baehr, J. Marotzke, J. Stark, S. A. Cunningham, and J.-O. Beismann, A monitoring design for the Atlantic meridional overturning circulation, Geophysical Research Letters, 30, 1413, 2003.

Jacobs, G. A., C. N. Barron, and R. C. Rhodes, Mesoscale characteristics, Journal of Geophysical Research, 106(C9), 19,581-19,595, doi:10.1029/2000JC000669, 2001.

Jayne, S. R., et al., The Kuroshio Extension and its recirculation gyres, Deep Sea Research, 56(12), 2088-2099, doi:10.1016/j.dsr.2009.08.006, 2009. 
Keller, J. B., and G. Veronis, Rossby waves in the presence of random currents, Journal of Geophysical Research, 74 (8), 1941-1951, doi:10.1029/JB074i008p01941, 1969.

Killworth, P. D., An equivalent-barotropic mode in the Fine Resolution Antarctic Model, Journal of Physical Oceanography, 22(11), 1379-1387, 1992.

Killworth, P. D., and J. R. Blundell, The effect of bottom topography on the speed of long extratropical planetary waves, Journal of Physical Oceanography, 29(10), 2689-2710, 1999.

Killworth, P. D., and J. R. Blundell, Large-scale propagating disturbances: Approximation by vertical normal modes, Journal of Physical Oceanography, 31(10), 28522870, 2001.

Killworth, P. D., and J. R. Blundell, The dispersion relation for planetary waves in the presence of mean flow and topography. Part I: Analytical theory and onedimensional examples, Journal of Physical Oceanography, 34 (12), 2692-2711, 2004.

Killworth, P. D., and J. R. Blundell, The dispersion relation for planetary waves in the presence of mean flow and topography. Part II: Two-dimensional examples and global results, Journal of Physical Oceanography, 35(11), 2110-2133, 2005.

Killworth, P. D., and J. R. Blundell, Planetary wave response to surface forcing and instability in the presence of mean flow and topography, Journal of Physical Oceanography, 37(5), 1297-1320, 2007.

Killworth, P. D., D. B. Chelton, and R. A. de Szoeke, The speed of observed and theoretical long extratropical planetary waves, Journal of Physical Oceanography, 27(9), 1946-1966, 1997.

Kubokawa, A., Quasigeostrophic planetary waves in a two-layer ocean with onedimensional periodic bottom topography, Geophysical and Astrophysical Fulid Dynamics, 102(2), 119-137, doi:10.1080/03091920701614334, 2008.

LaCasce, J. H., Surface quasigeostrophic solutions and baroclinic modes with exponential stratification, Journal of Physical Oceanography, 42(4), 569-580, doi: 10.1175/JPO-D-11-0111.1, 2012.

LaCasce, J. H., and J. Pedlosky, The instability of Rossby basin modes and the oceanic eddy field, Journal of Physical Oceanography, 34 (9), 2027—2041, 2004.

Lapeyre, G., and P. Klein, Dynamics of the upper oceanic layers in terms of surface quasigeostrophy theory, Journal of Physical Oceanography, 36 (2), 165-176, 2006.

Le Traon, P.-Y., P. Gaspar, F. Bouyssel, and H. Makhmara, Using TOPEX/POSEIDON data to enhance ERS-1 data, Journal of Atmospheric and Oceanic Technology, 12(1), 161-170, 1995. 
Le Traon, P.-Y., F. Nadal, and N. Ducet, An improved mapping method of multisatellite altimeter data, Journal of Atmospheric and Oceanic Technology, 15(2), $522-534,1998$.

Le Traon, P.-Y., Y. Faugère, F. Hernandez, J. Dorandeu, F. Mertz, and M. Albain, Can we merge GEOSAT follow-on with TOPEX/poseidon and ERS-2 for an improved description of the ocean circulation?, Journal of Atmospheric and Oceanic Technology, 20(6), 889-895, 2003.

Ledwell, J. R., A. J. Watson, and L. S. Clifford, Mixing of a tracer in the pycnocline, Journal of Geophysical Research, 103(C10), 21,499-21,529, 1998.

Lvov, Y. V., K. L. Polzin, and E. G. Tabak, Energy spectra of the ocean's internal wave field: theory and observations, Physical Review Letters, 92(12), 2004.

Maharaj, A. M., P. Cipollini, N. J. Holbrook, P. D. Killworth, and J. R. Blundell, An evaluation of the classical and extended Rossby wave theories in explaining spectral estimates of the first few baroclinic modes in the South Pacific Ocean, Ocean Dynamics, 57(3), 173-187, 2007.

Maltrud, M. E., and J. L. McClean, An eddy resolving global 1/10 ocean simulation, Ocean Modelling, 8(1-2), 31-54, 2005.

Marshall, J., A. Adcroft, C. Hill, L. Perelman, and C. Heisey, A finite-volume, incompressible Navier Stokes model for studies of the ocean on parallel computers, Journal of Geophysical Research, 102(C3), 5753-5766, 1997.

Maximenko, N. A., B. Bang, and H. Sasaki, Observational evidence of alternating zonal jets in the world ocean, Geophysical Research Letters, 32, L12,607, 2005.

McWilliams, J. C., and G. R. Flierl, On the evolution of isolated, nonlinear vortices, Journal of Physical Oceanography, 9(6), 1155-1182, 1979.

Menemenlis, D., I. Fukumori, and T. Lee, Using Green's functions to calibrate an ocean general circulation model, Monthly Weather Review, 133(5), 1224-1240, 2005.

Menemenlis, D., J.-M. Campin, P. Heimbach, C. Hill, T. Lee, A. Nguyen, M. Schodlok, and H. Zhang, ECCO2: High resolution global ocean and sea ice data synthesis, Mercator Ocean Quarterly Newsletter, 31, 13-21, 2008.

Müller, P., and C. Frankignoul, Direct atmospheric forcing of geostrophic eddies, Journal of Physical Oceanography, 11 (3), 287-308, 1981.

Nakano, H., and H. Hasumi, A series of zonal jets embedded in the broad zonal flows in the Pacific obtained in eddy-permitting ocean general circulation models, Journal of Physical Oceanography, 35(4), 474-488, 2005. 
Penduff, T., B. Barnier, J.-M. Mollines, and G. Madec, On the use of current meter data to assess the realism of ocean model simulations, Ocean Modelling, 11(3-4), 399-416, 2006.

Philander, S. G. H., Forced oceanic waves, Reviews of Geophysics, 16(1), 15-46, 1978.

Ponte, R. M., C. Wunsch, and D. Stammer, Spatial mapping of time-variable errors in jason-1 and TOPEX/Poseidon sea surface height measurements, Journal of Atmospheric and Oceanic Technology, 24 (6), 1078-1085, 2007.

Press, W. H., S. A. Teukolsky, W. T. Vetterling, and B. P. Flannery, Numerical Recipes: The art of scientific computing, 3rd ed., Cambridge University Press, New York, 2007.

Purkey, S. G., and G. C. Johnson, Warming of global abyssal and deep Southern Ocean waters between the 1990s and 2000s: Contributions to global heat and sea level rise budgets, Journal of Climate, 23(23), 6336-6351, 2010.

Reznik, G. M., and T. B. Tsybaneva, Planetary waves in a stratified ocean of variable depth. Part 1. two-layer model, Journal of Fluid Mechanics, 388, 115-145, 1999.

Rhines, P. B., Edge-, bottom-, and Rossby waves in a rotating stratified fluid, Geophysical and Astrophysical Fulid Dynamics, 1(3), 273-302, 1970.

Rhines, P. B., Waves and turbulence on a beta-plane, Journal of Fluid Mechanics, 69, 417-443, 1975.

Rhines, P. B., and F. P. Bretherton, Topographic Rossby waves in a rough-bottomed ocean, Journal of Fluid Mechanics, 61 (3), 583-607, 1973.

Richards, K. J., N. A. Maximenko, F. O. Bryan, and H. Sasaki, Zonal jets in the Pacific Ocean, Geophysical Research Letters, 33, L03,605, 2006.

Richman, J. G., C. Wunsch, and N. G. Hogg, Space and time scales of mesoscale motion in the western North Atlantic, Reviews of Geophysics, 15(4), 385-420, 1977.

Rio, M. H., P. Schaeffer, F. Hernandez, and J.-M. Lemoine, The estimation of the ocean Mean Dynamic Topography through the combination of altimetric data, insitu measurements and GRACE geoid: From global to regional studies, in paper presented at GOCINA International Workshop, European Center for Geodynamics and Seismology, Luxembourg, 2005.

Rio, M. H., S. Guinehut, and G. Larnicol, New CNESCLS09 global mean dynamic topography computed from the combination of GRACE data, altimetry, and in situ measurements, Journal of Geophysical Research, 116, C07,018, doi: 10.1029/2010JC006505, 2011. 
Roden, G., Shipboard acoustic doppler current profiling during cruise TT9306 (SAC ID 00016), doi:10.1594/PANGAEA.319488, 2005.

Roemmich, D., and B. Cornuelle, Observing the fluctuations of gyre-scale ocean circulation: A study of the subtropical South Pacific, Journal of Physical Oceanography, 20(12), 1919-1934, doi:10.1175/1520-0485(1990)020<1919:OTFOGS>2.0.CO;2, 1990.

Samelson, R. M., Surface-intensified Rossby waves over rough topography, Journal of Marine Research, 50(3), 367-384, 1992.

Scharffenberg, M., and D. Stammer, Statistical parameters of the geostrophic ocean flow field estimated from the Jason-1-TOPEX/Poseidon tandem mission, Journal of Geophysical Research, 116, C12,011, 2011.

Schmitz, W. J., Jr., Observations of the vertical distribution of low frequency kinetic energy in the Western North Atlantic, Journal of Marine Research, 36 (2), 295-310, 1978.

Schmitz, W. J., Jr., Exploration of the eddy field in the midlatitude North Pacific, Journal of Physical Oceanography, 18(3), 459-468, doi:10.1175/15200485(1988)018<0459:EOTEFI >2.0.CO;2, 1988.

Scott, R. B., and D. G. Furnival, Assessment of traditional and new eigenfunction bases applied to extrapolation of surface geostrophic current time series to below the surface in an idealized primitive equation simulation, Journal of Physical Oceanography, 42(1), 165-178, 2012.

Scott, R. B., and F. Wang, Direct evidence of an oceanic inverse kinetic energy cascade from satellite altimetry, Journal of Physical Oceanography, 35(9), 1650-1666, 2005.

Scott, R. B., B. K. Arbic, E. P. Chassignet, A. C. Coward, M. Maltrud, A. Srivivasan, and A. Varghese, Total kinetic energy in three global eddying ocean circulation models and over 5000 current meter records, Ocean Modelling, 32(3-4), 157-169, 2010.

Smith, K. S., The geography of linear baroclinic instability in Earth's oceans, Journal of Marine Research, 65(5), 655-683, 2007.

Smith, K. S., and G. K. Vallis, The scales and equilibration of midocean eddies: Freely evolving flow, Journal of Physical Oceanography, 31(2), 554-571, 2001.

Smith, K. S., and J. Vanneste, A surface-aware projection basis for quasigeostrophic flow, submitted to Journal of Physical Oceanography, 2012.

Smith, W. H. F., and D. T. Sandwell, Global sea floor topography from satellite altimetry and ship depth soundings, Science, 277 (5334), 1956-1962, 1997. 
Stammer, D., Global characteristics of ocean variability estimated from regional TOPEX/POSEIDON altimeter measurements, Journal of Physical Oceanography, 27(8), 1743-1769, 1997.

Stammer, D., and C. W. Böning, Mesoscale variability in the atlantic ocean from Geosat altimetry and WOCE high-resolution numerical modeling, Journal of Physical Oceanography, 22(7), 732-752, 1992.

Sura, P., and S. T. Gille, Stochastic dynamics of sea surface height variability, Journal of Physical Oceanography, 40(7), 1582-1596, 2010.

Tailleux, R., and J. C. McWilliams, The effect of bottom pressure decoupling on the speed of extratropical, baroclinic Rossby waves, Journal of Physical Oceanography, $31(6), 1461-1476,2001$.

Thompson, A. F., and W. R. Young, Scaling baroclinic eddy fluxes: Vortices and energy balance, Journal of Physical Oceanography, 36, 720-738, 2006.

Tulloch, R., J. Marshall, and K. S. Smith, Interpretation of the propagation of surface altimetric observations in terms of planetary waves and geostrophic turbulence, Journal of Geophysical Research, 114(C02005), doi:10.1029/2008JC005055, 2009.

Tulloch, R., J. Marshall, C. Hill, and K. S. Smith, Scales, growth rates and spectral fluxes of baroclinic instability in the ocean, Journal of Physical Oceanography, $41(6), 1057-1076,2011$.

Vanneste, J., Nonlinear dynamics over rough topography: homogeneous and stratified quasi-geostrophic theory, Journal of Fluid Mechanics, 474, 299-318, 2003.

Wang, D.-P., C. N. Flagg, K. Donohue, and H. T. Rossby, Wavenumber spectrum in the Gulf Stream from shipboard ADCP observations and comparison with altimetry measurements, Journal of Physical Oceanography, 40(4), 840-844, doi: 10.1175/2009JPO4330.1, 2010.

Wunsch, C., The vertical partition of oceanic horizontal kinetic energy, Journal of Physical Oceanography, 27, 1770-1794, 1997.

Wunsch, C., A summary of North Atlantic baroclinic variability, Journal of Physical Oceanography, 29(12), 3161-3166, doi:10.1175/15200485(1999)029<3161:ASONAB>2.0.CO;2, 1999.

Wunsch, C., Discrete Inverse and State Estimation Problems with Geophysical Fluid Applications, Cambridge University Press, 2006.

Wunsch, C., Mass and volume transport variability in an eddy-filled ocean, Nature Geoscience, 1(3), 165-168, 2008.

Wunsch, C., The oceanic variability spectrum and transport trends, AtmosphereOcean, 47(4), 281-291, 2009. 
Wunsch, C., Toward a mid-latitude ocean frequency-wavenumber spectral density and trend determination, Journal of Physical Oceanography, 40, 2264-2281, 2010.

Wunsch, C., and D. Stammer, The global frequency-wavenumber spectrum of oceanic variability estimated from TOPEX/POSEIDON altimetric measurements, Journal of Geophysical Research, 100(C12), 24,895-24,910, 1995.

Wunsch, C., and D. Stammer, Atmospheric loading and the oceanic "inverted barometer" effect, Reviews of Geophysics, 35(1), 79-107, 1997.

$\mathrm{Xu}, \mathrm{Y}$., and L.-L. Fu, Global variability of the wavenumber spectrum of oceanic mesoscale turbulence, Journal of Physical Oceanography, 41(4), 802-809, 2011.

$\mathrm{Xu}, \mathrm{Y}$., and L.-L. Fu, The effects of altimeter instrument noise on the estimation of the wavenumber spectrum of sea surface height, in preparation, 2012.

Zang, X., Spectral description of low frequency oceanic variability, Ph.D. thesis, MIT/WHOI Joint Program, 2000.

Zang, X., and C. Wunsch, The observed dispersion relationship for North Pacific Rossby wave motions, Journal of Physical Oceanography, 29(9), 2183-2190, 1999.

Zang, X., and C. Wunsch, Spectral description of low-frequency oceanic variability, Journal of Physical Oceanography, 31(10), 3073-3095, 2001. 